SLAC-R-537

$\mathrm{UC}-414$

\title{
Hadronic Charmless B Decays at the SLD*
}

\author{
P. L. Reinertsen \\ Stanford Linear Accelerator Center \\ Stanford University \\ Stanford, CA 94309
}

SLAC-Report-537

May 1999

Prepared for the Department of Energy

under contract number DE-AC03-76SF00515

Printed in the United States of America. Available from the National Technical Information Service, U.S. Department of Commerce, 5285 Port Royal Road, Springfield, VA 22161.

${ }^{*}$ Ph.D. thesis, University of California, Santa Cruz. 


\section{Contents}

Abstract $\quad$ iv

Dedication $\quad$ v

Acknowledgements vi

List of Figures viii

List of Tables $\quad$ xi

1 Introduction $\quad \mathbf{1}$

1.1 The Standard Model .................... 3

1.1.1 The Standard Electroweak Theory . . . . . . . . . . 6

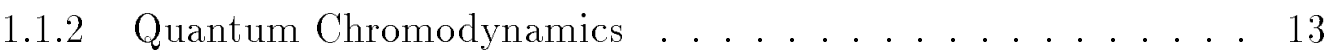

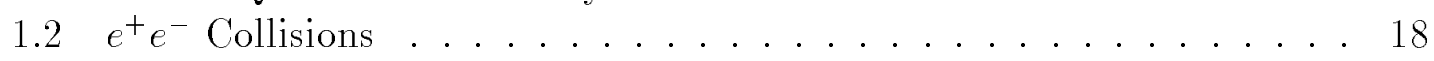

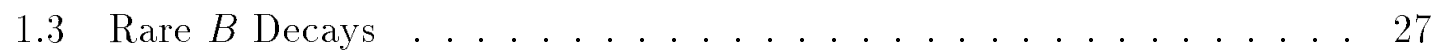

1.3.1 The Search Modes at SLD .............. 31

2 The Accelerator $\quad 37$

2.1 Stanford Linear Collider . . . . . . . . . . . . . . . 39

2.1.1 Linear Accelerator . . . . . . . . . . . . 41

2.1.2 Luminosity Overview .................. . . . . . . . . . . . . 41

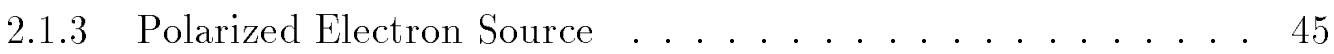

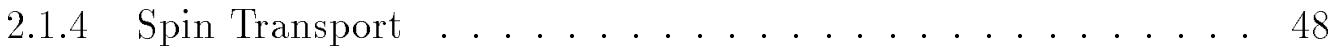

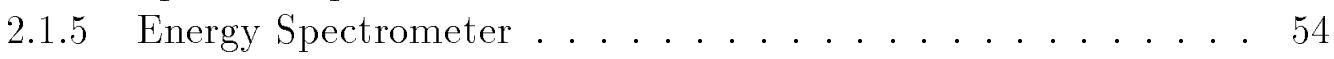


Contents

2.2 Compton Polarimeter. . . . . . . . . . . . . . . . 56

2.2 .1 Compton Laser . . . . . . . . . . . . . . . . 56

2.2 .2 Electron Transport . . . . . . . . . . . . . . . 58

2.2 .3 Compton Čerenkov Detector . . . . . . . . . . . . . 59

2.2.4 The Polarization Measurement. . . . . . . . . . . . . 61

3 The SLD Detector $\quad 67$

3.1 Luminosity Monitor . . . . . . . . . . . . . . . . 70

3.2 The Vertex Detectors . . . . . . . . . . . . . . . . . 73

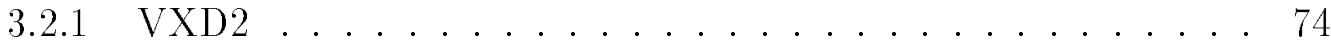

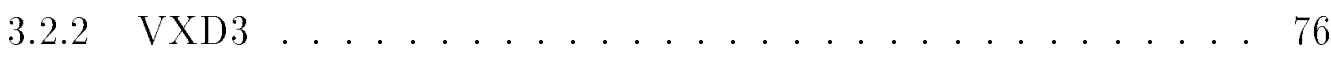

3.3 Central Drift Chamber . . . . . . . . . . . . . 80

3.3.1 Track Reconstruction . . . . . . . . . . . . . . . 85

3.4 Cerenkov Ring Imaging Detector . . . . . . . . . . . . . . . 92

3.5 Liquid Argon Calorimeter . . . . . . . . . . . . . . . . 96

3.6 Warm Iron Calorimeter . . . . . . . . . . . . . . . . . 101

3.7 Data Acquisition . . . . . . . . . . . . . . . . . 103

3.8 Detector Simulation. . . . . . . . . . . . . . . . . . . 104

4 Candidate Vertices and MC Calibration 107

4.1 Detector Trigger. . . . . . . . . . . . . . . . 108

4.2 Candidate Vertices, a A Second Stage Filter . . . . . . . . . . 113

4.3 Calibration of the MC . . . . . . . . . . . . . 123

4.3.1 Tracking Efficiency . . . . . . . . . . . . . . . . . . . 124

4.3.2 Momentum Smearing . . . . . . . . . . . . . . . 128

4.3.3 Vertexing Resolution . . . . . . . . . . . . . . . . . 131

5 The Rare Decay Event Selection Algorithm 135

5.1 Parameters . . . . . . . . . . . . . . . . . 136

5.2 Direct cuts . . . . . . . . . . . . . . . . . . . . 144

5.3 Discriminator Functions . . . . . . . . . . . . . . . . 151

5.4 Optimal Point of Analysis . . . . . . . . . . . . . . . 157

5.5 Cross Check Mode . . . . . . . . . . . . . . . . 163

5.6 The Case Against Using Particle ID . . . . . . . . . . . . . 165 
Contents

6 Data and Final Results 173

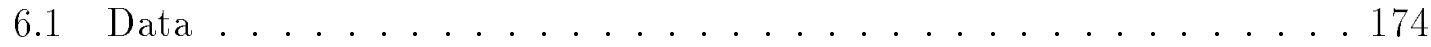

6.2 Limits and Uncertainties . . . . . . . . . . . . . 181

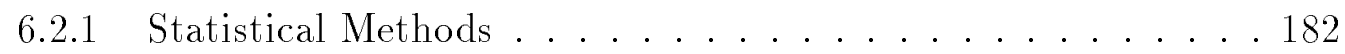

6.2 .2 Uncertainties . . . . . . . . . . . . . 188

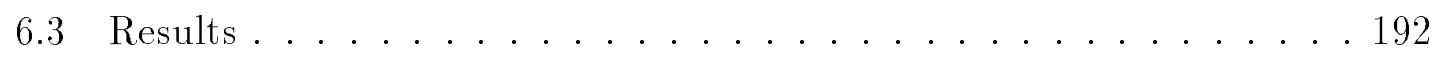

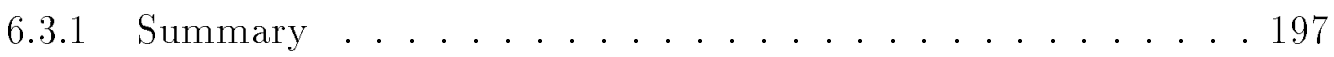

A The SLD Collaboration $\quad 201$

B Backup Plots Associated with the Main Text 205

$\begin{array}{ll}\text { Bibliography } & 235\end{array}$ 


\author{
Abstract \\ Hadronic Charmless B decays at the SLD \\ by \\ Per Lasse Reinertsen
}

Rare decays of beauty particles were studied in several two-body exclusive hadronic charmless modes using the $19.4 p b^{-1} Z$-pole data collected with the SLD detector at SLAC from 1993 to 1998 . These decays are mediated by both tree level $b \rightarrow u$ and one-loop penguin $b \rightarrow s, d$ transitions. Upper limits for the branching ratios are set for the investigated modes $B_{s}, B^{0} \rightarrow P^{+} P^{-}, B^{+} \rightarrow V P^{+}$and $B_{s}, B^{0} \rightarrow V V$, where the pseudoscalar particle $P^{+}$is either $\pi^{+}$or $K^{+}$and the vector particle $V$ is either $\rho^{0}, K^{* 0}$ or $\phi$. Using an event selection algorithm consisting of a set of hard cuts combined with a set of discriminator functions, the efficiencies range between $24 \%$ and $37 \%$ with near zero background. 
Anne 


\section{Acknowledgements}

First and foremost I would like to thank my advisor Bruce Schumm, for his guidance, encouragement and friendship, for his excellent humor and for numerous discussions about physics, stockmarket, hiking and life in general. Without him this analysis wouldn't exist.

I owe a tremendous debt to the friends I have made working on the Compton polarimeter. Working in this group has been very challenging and rewarding. Besides of ski trips, hiking and cookies, I like to thank Mike Woods for his constant effort to ensure the well being of the polarimeter measurement, he made me challenge and question the measurement in a way that was both frustrating and highly productive. I would like to thank Morris Swartz for providing me with several plots and figures that I used in my electroweak presentations and for his excellent knowledge from which I have benefited. I would like to thank Peter Rowson for being an excellent leader of the electroweak group, for his down to earth style and great diplomatic skills, his endless visits to my office have been highly appreciated. Thanks to Jorge Fernandez, my fellow student and officemate, for lending me his ski's, for social get togethers, for several interesting discussions and for being such a pleasant officemate. I owe quite a lot to the former student Eric Torrence for rapidly bringing me up to 
date on the do's and dont's at the SLD and for teaching me how to work the intricate data processing system.

In the same breath I would also like to thank the computer literates Richard Dubois, Gary Bower, Tony Johnson and Matt Langston for their help on my, what must have been annoying, inquiries. Special thanks are extended to Mohan Kalelkar for providing prompt delivery of specially generated MC events used in this analysis.

I would like to further thank Mourad Daoudi, Glen Crawford, Su Dong, David Muller, Stephane Willocq, Masako Iwasaki and John Coller for valuable inputs to the analysis herein.

My stay in Santa Cruz has been very enjoyable and constructive. I would like on a general note to thank the entire physics community for a laid back albeit high quality environment. Special thanks goes to Wilko Kroger for his patient and highly valuable help on computer issues and to Nora Rogers, Georgia Hamel, Marilyn Stevens, Judy Hurst and Terry Hart who have always been very helpful with administrative matters.

I would like to thank all my friends who I have spent so much great time with in Santa Cruz, and I would like to thank my friends and family in Norway for always believing in me. You have all been a great inspiration to me. 
viii

\section{List of Figures}

1.1 Running of the Strong Coupling Constant . . . . . . . . . . . 17

1.2 Feynman Diagrams for $e^{+} e^{-} \rightarrow f \bar{f} \ldots \ldots \ldots 18$

1.3 QED Radiative Corrections to $e^{+} e^{-} \rightarrow f \bar{f} \ldots \ldots . . . . . . .19$

1.4 Oblique Corrections to $e^{+} e^{-} \rightarrow f \bar{f} \ldots \ldots \ldots 20$

1.5 Quark Hadronization ................. 21

1.6 Hadron Production . . . . . . . . . . . . . . 22

1.7 Hadron Cross Section . . . . . . . . . . . . . . . 24

1.8 Rare $B$ Decay, Radiative and Dineutrino Modes . . . . . . . . . . 27

1.9 Rare $B$ Decay, Hadronic Modes . . . . . . . . . . . . . . 28

2.1 SLC Layout . . . . . . . . . . . . . . . . . . 40

2.2 Enhancement Factor . . . . . . . . . . . . . . 42

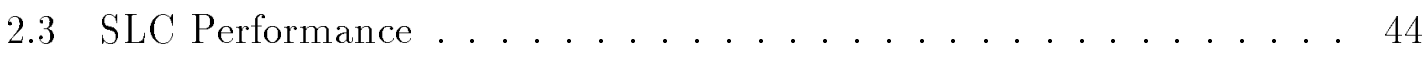

2.4 Gallium Arsenide Energy Levels . . . . . . . . . . . . . . 46

2.5 Polarized Electron Gun and Laser . . . . . . . . . . . . . . 47

2.6 SLC Polarization History . . . . . . . . . . . . . . . 50

2.7 Spin Bumps . . . . . . . . . . . . . . . 51

2.8 Polarization, Energy Dependence . . . . . . . . . . 53

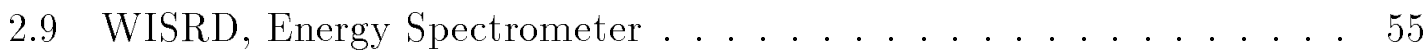

2.10 Compton Polarimeter. . . . . . . . . . . . . 57

2.11 Compton Polarimeter Spectrometer . . . . . . . . . . . 59

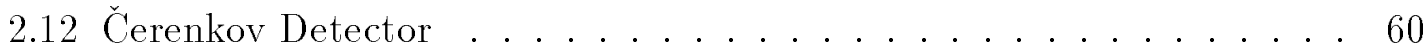

2.13 Compton Asymmetry . . . . . . . . . . . . 63

3.1 SLD Detector, Isometric Profile . . . . . . . . . . . . 68 


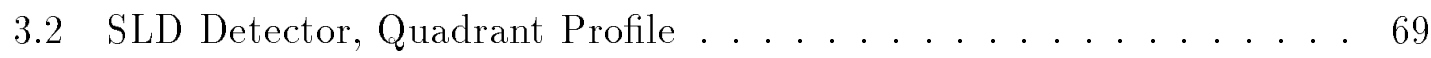

3.3 Luminosity Monitor. . . . . . . . . . . . . . . . 70

3.4 A Luminosity Monitor Layer . . . . . . . . . . . . . . . . 71

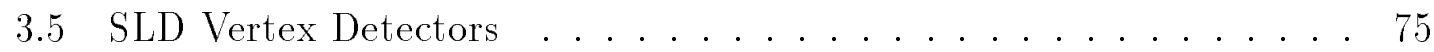

3.6 Resolution Parameters for the SLD Vertex Detectors . . . . . . . 77

3.7 A VXD3 Detector Ladder . . . . . . . . . . . . . . . 78

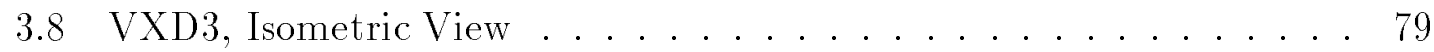

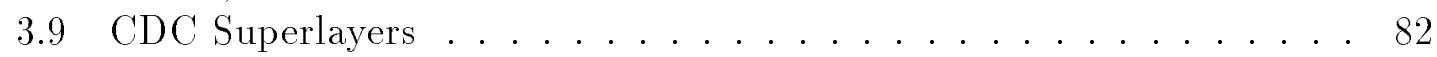

3.10 Drift Cell Layout in the CDC . . . . . . . . . . . . 83

3.11 CDC Field Map . . . . . . . . . . . . . . . . . 84

3.12 Track Reconstruction . . . . . . . . . . . . . . 86

3.13 Distribution of Number of Hits per Track . . . . . . . . . . . . . 88

3.14 Hit Finding Efficiency as a Function of CDC Wire Layer . . . . . . 89

3.15 Drift Distance Resolution in CDC . . . . . . . . . . . . . . 90

3.16 CDC, $\cos \theta$ Range . . . . . . . . . . . . . . 91

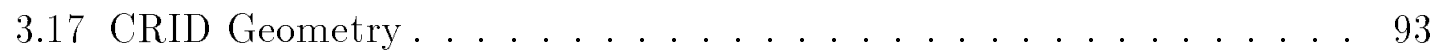

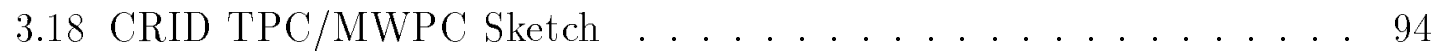

3.19 LAC Barrel Module . . . . . . . . . . . . . . . 97

3.20 LAC Detail . . . . . . . . . . . . . . . . . 98

3.21 LAC Endcap Module . . . . . . . . . . . . . . . . 99

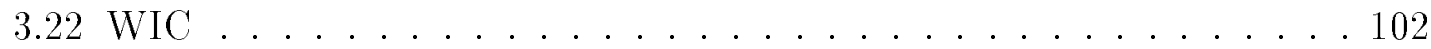

4.1 A Two Jet Event . . . . . . . . . . . . . . . . . . 114

4.2 Peterson Fragmentation Function . . . . . . . . . . 116

4.3 Fragmentation Parameter and Track Spread . . . . . . . . . . . 117

4.4 Stiff Track Distribution and Vertex Mass . . . . . . . . . . . . . . . 118

4.5 Vertex Significance for Background Modes and Search Modes . . . . . 120

4.6 Classification of Tracks . . . . . . . . . . . . . . 125

4.7 Track Multiplicity MC/Data, Transverse Momentum . . . . . . . . 127

4.8 Track Multiplicity MC/Data, Polar Angle . . . . . . . . . . . . 128

$4.9 D^{+} \rightarrow K^{-} \pi^{+} \pi^{+}$, Mass Width before Smearing . . . . . . . . . . . . 129

$4.10 D^{+} \rightarrow K^{-} \pi^{+} \pi^{+}$, Mass Width after Smearing . . . . . . . . . . . 130

4.11 Vertex Mass, Effect of $p_{t}$ Smearing . . . . . . . . . . . . 131

4.12 Track Coordinates at POCA . . . . . . . . . . . . 132

4.13 Track Impact Parameter, Effect of Vertex Resolution Smearing . . . . 133

5.1 Mass Assignment for $B^{0} \rightarrow \phi \rho^{0} \ldots \ldots \ldots$. . . . . . . . 137 
5.2 Reconstructed Masses I . . . . . . . . . . . . . . . . . 138

5.3 Reconstructed Masses II . . . . . . . . . . . . . . . . 139

5.4 Reconstructed Vertex Probability and Track Spread . . . . . . . . 140

5.5 Impact Parameters . . . . . . . . . . . . . . . . . . 141

5.6 Reconstructed Impact Parameters . . . . . . . . . . . . . . . 142

5.7 Reconstructed Helicity Angle and Mass Measure for $B \rightarrow P V$. . . 143

5.8 Reconstructed Mass Measures for $B \rightarrow P P$ and $B \rightarrow V V$. . . . . 145

5.9 Evolution of Mass Distribution I . . . . . . . . . . . . . . 148

5.10 Evolution of Mass Distribution II . . . . . . . . . . . . . . 149

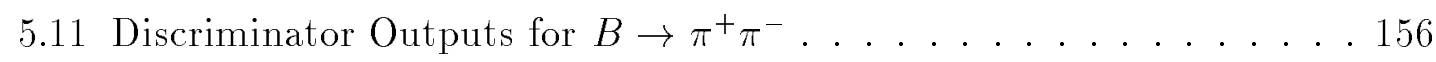

5.12 Background and Efficiency for $B \rightarrow \pi^{+} \pi^{-}$. . . . . . . . . . . 157

5.13 Average Poisson Limit and Efficiency for $B^{0} \rightarrow \pi^{+} \pi^{-}$. . . . . . 158

$5.14 D^{+} \rightarrow K^{-} \pi^{+} \pi^{+}$, Mass Distribution . . . . . . . . . . . 164

6.1 Background Comparison, Data and MC ............................. 177

6.2 Event Display . . . . . . . . . . . . . . . . 179

6.3 Classical Confidence Interval . . . . . . . . . . . . . . 187

6.4 Probability Distribution for Delphi-SLD Difference . . . . . . . 197

B.1 Discriminator Outputs for VXD2 and $B \rightarrow P P \ldots 206$

B.2 Discriminator Outputs for VXD3 and $B \rightarrow P P \ldots 207$

B.3 Discriminator Outputs for VXD2 and $B \rightarrow P V \ldots . . . . . .208$

B.4 Discriminator Outputs for VXD3 and $B \rightarrow P V \ldots . . .209$

B.5 Discriminator Outputs for VXD2 and $B^{0} \rightarrow V V \ldots \ldots 210$

B.6 Discriminator Outputs for VXD3 and $B^{0} \rightarrow V V \ldots \ldots . . . . . .211$

B.7 Discriminator Outputs for VXD2 and $B_{s} \rightarrow V V \ldots \ldots . \ldots 212$

B.8 Discriminator Outputs for VXD3 and $B_{s} \rightarrow V V \ldots \ldots 213$

B.9 Background Versus Efficiency for $B \rightarrow P P($ VXD2) . . . . . . . 214

B.10 Background Versus Efficiency for $B \rightarrow P P($ VXD3) . . . . . . . 215

B.11 Background Versus Efficiency for $B \rightarrow P V$ (VXD2) . . . . . . . . 216

B.12 Background Versus Efficiency for $B \rightarrow P V$ (VXD3) . . . . . . . 217

B.13 Background Versus Efficiency for $B^{0} \rightarrow V V$ (VXD2) . . . . . . 218

B.14 Background Versus Efficiency for $B^{0} \rightarrow V V$ (VXD3) . . . . . . 219

B.15 Background Versus Efficiency for $B_{s} \rightarrow V V$ (VXD2) . . . . . . . 220

B.16 Background Versus Efficiency for $B_{s} \rightarrow V V$ (VXD3) . . . . . . . . 221

B.17 Average Poisson Limit Versus Efficiency for $B \rightarrow P P$. . . . . . . 222

B.18 Average Poisson Limit Versus Efficiency for $B \rightarrow P V$. . . . . . 223 
B.19 Average Poisson Limit Versus Efficiency for $B^{0} \rightarrow V V$. . . . . . . 224

B.20 Average Poisson Limit Versus Efficiency for $B_{s} \rightarrow V V$. . . . . . 225

B.21 Background Comparison for $B \rightarrow P P$ (VXD2) . . . . . . . . 226

B.22 Background Comparison for $B \rightarrow P V$ (VXD2) . . . . . . . . . 227

B.23 Background Comparison for $B^{0} \rightarrow V V$ (VXD2) . . . . . . . . 228

B.24 Background Comparison for $B_{s} \rightarrow V V$ (VXD2) . . . . . . . . 229

B.25 Background Comparison $B \rightarrow P P$ (VXD3) . . . . . . . . . 230

B.26 Background Comparison for $B \rightarrow P V$ (VXD3) . . . . . . . . . 231

B.27 Background Comparison for $B^{0} \rightarrow V V$ (VXD3) . . . . . . . . 232

B.28 Background Comparison for $B_{s} \rightarrow V V$ (VXD3) . . . . . . 233 


\section{List of Tables}

1.1 Fermions in the Electroweak Model . . . . . . . . . . . . 7

1.2 Hadronic Cross Section in $e^{+} e^{-}$Collisions . . . . . . . . . 25

1.3 Number of $B$ Mesons at SLD . . . . . . . . . . . . 26

1.4 Search Modes at SLD . . . . . . . . . . . . . . . . 32

1.5 Rare B Decay, Quark Content . . . . . . . . . . 33

1.6 Branching Ratio, Prediction and Measurements . . . . . . . . 35

2.1 SLC Collision Beam Parameters . . . . . . . . . . . . . 43

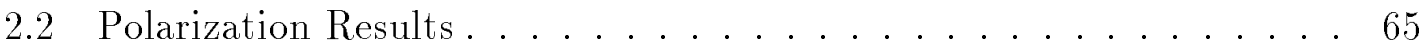

2.3 Systematic Polarization Effects ............. 66

3.1 SLC Luminosity Results . . . . . . . . . . . . . . 72

4.1 Generated Search Modes . . . . . . . . . . . . . . . . 122

4.2 Weight Constants Applied to the MC Tracks . . . . . . . . . 126

5.1 List of Direct Cuts . . . . . . . . . . . . . . 147

5.2 Background Level and Efficiency, Direct Cuts . . . . . . . . . . 150

5.3 Parameters for the $B \rightarrow P P$ Discriminator Functions . . . . . . . 152

5.4 Parameters for the $B \rightarrow P V$ Discriminator Functions . . . . . . . . 153

5.5 Parameters for the $B^{0} \rightarrow V V$ Discriminator Functions . . . . . . . 154

5.6 Parameters for the $B_{s} \rightarrow V V$ Discriminator Functions . . . . . . . 155

5.7 Efficiency and Background at Optimal Point of Analysis I . . . . . 159

5.8 Efficiency and Background at Optimal Point of Analysis II . . . . . . 160

5.9 Background Events I . . . . . . . . . . . . . . 161

5.10 Background events II . . . . . . . . . . . . . . . 162 
5.11 CRID Particle ID, Purity and Efficiency . . . . . . . . . 167

5.12 Effect of CRID Particle ID I . . . . . . . . . . . . . . . . . 168

5.13 Effect of CRID Particle ID II . . . . . . . . . . . . . . . . 169

5.14 Effect of $e$ and $\mu$ Particle ID . . . . . . . . . . . . . . 171

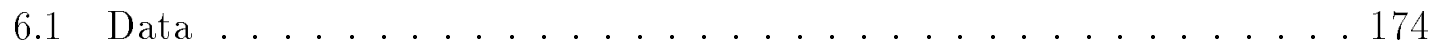

6.2 Parameter List for Data Events . . . . . . . . . . . . . 175

6.3 Systematic Effects I . . . . . . . . . . . . . . . . 189

6.4 Systematic Effects II . . . . . . . . . . . . . . . . 190

6.5 Sensitivity, $S$ and $\sigma_{s}$ I . . . . . . . . . . . 191

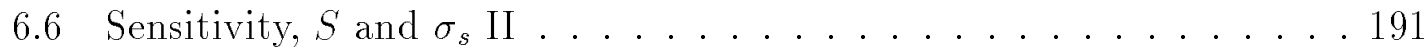

6.7 Bayesian $90 \%$ Confidence Level Upper Limits . . . . . . . . . . . 192

$6.890 \%$ Classical Confidence Intervals I . . . . . . . . . . . . . . 193

$6.990 \%$ Classical Confidence Intervals II . . . . . . . . . . . . . . 194

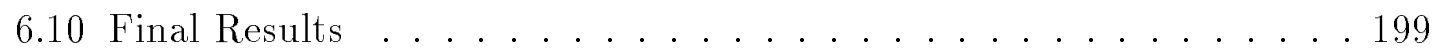





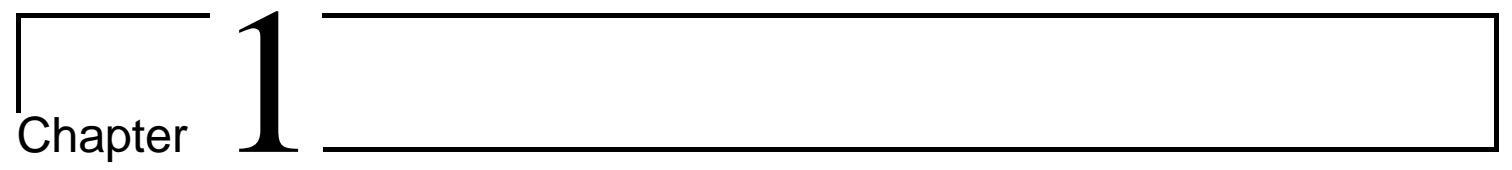

\section{Introduction}

During the last 100 years our understanding of the world has changed dramatically. At the time when the electron was discovered (Thomson 1897), there was still a general belief in the validity of classical physics in which particles and waves were totally separate concepts. It is really no more than a historical accident that electrons were first observed in an experiment in which they behaved like classical particles, while experiments in which electron beams show a wave-like character were not carried out until much later (Davisson 1927, Thomson 1927). In the case of light quanta, the wave nature of light was a dogma of the nineteenth century. It was only with the discovery of the photo electric effect (Einstein 1905) that physicists where 


\section{Introduction}

forced to think again. Approximately 30 years later it had been realized that particles and waves cannot be regarded as two separate phenomena, but must instead be considered as two aspects of the same phenomena. This and the theory of special relativity (Einstein 1905) paved the way for modern physics.

Today, the formulation of the interaction between fundamental particles and the forces is embedded in what we call the Standard Model (SM) which consists of the electroweak sector and the strong sector (QCD). Three of the major themes of modern physics, the quantum gauge theory, the field concept and the principle of special relativity are all a natural part of the formalism. The SM has been verified with ever increasing accuracy by the large data samples currently being collected at the many high energy accelerators running at places such as FNAL, KEK, CERN, SLAC and CESR. While this may be a theoretical triumph for the SM, theorists of today adamantly believe that there must be something beyond this model which is more comprehensive and complete and that unifies the strong force, the electroweak force and the non-standard model gravitational force. A number of models have been proposed in recent years and the search for unified theories and signatures of such theories are driving the particle physics community today. Since the current body of experimental data agrees quite well with the Standard Model, however, there are few experimental guidances available to distinguish which, if any of the various flavors 


\subsection{The Standard Model}

of extended theories is correct. This has left the particle physics community quite interested in finding any sort of experimental signature of something new that the Standard Model cannot accommodate.

Loop structures in $B$ decays may be our most sensitive probe of physics beyond the standard model and this thesis looks for possible deviations from the SM predictions in the rate of rare hadronic $B$ decays. In the remainder of this chapter an overview of the SM will be given, followed by a discussion around the topic of rare $B$ decays, which also serves as a motivation for the analysis herein. Chapter 2 and 3 will give an overview of the SLC/SLD experiment, and in chapters 4 and 5 the event selection and the analysis are given. Chapter 6 lists the results together with cross-checks and conclusions.

\subsection{The Standard Model}

The subject of high-energy physics grew from the attempt to understand the nucleus, in particular to study the nature of the forces between protons and neutrons that hold the nucleus together. This study began in the 1930 s using cosmic rays as the source of high energy particles and it progressed forward with the invention of ac-

celerators which gave experimenters the possibility to do experiments in well-defined 


\section{Introduction}

conditions. This work unexpectedly led to the discovery of lots of new types of particles and it was not until high-energy accelerators probed deep within the neutron and proton that these many new particles were understood and physicists began to have a deep understanding of nuclear forces. There are new particle discoveries still being made today and there are now several hundred particles listed in the Particle Data Group Review 1998 [1]. The very rich particle spectrum is now understood as composite structures where the building blocks are the fundamental objects, the six quarks and the six leptons. All matter is composed of these fundamental objects as they interact via the exchange of gauge bosons. The forces that significantly affect them are the unified electroweak force, whose gauge bosons are the photon and the $W^{ \pm}$and $Z^{0}$ bosons, and the strong force. The theory of the strong force is called the quantum chromodynamics (QCD) and the corresponding gauge bosons are the eight gluons.

The construction of the SM is a fairly technical issue, but as for other areas of physics it is based on a few basic principles. In classical mechanics, for example, the physical laws are a set of rules that predict an objects motion exactly given the initial conditions and the force acting on the object. There are several ways of formulating the predictability of such motions and the most common, perhaps, is the explicit knowledge of the force and its impact on any object as stated in Newton's law 


\subsection{The Standard Model}

$F=m a$. Alternatively, one can construct a Hamiltonian which is nothing but the objects energy ( $H=E=$ Kinetic + Potential $)$ and provide a set of rules for how to extract information from this energy. This approach is commonly used in Quantum Mechanics, exemplified by the Schrödinger equation. Somewhat of the same flavor as the Hamiltonian formalism, the Lagrangian formalism uses the Lagrangian which is the Legendre transformation of the Hamiltonian ( $L=$ Kinetic-Potential $)$. The rule that governs the object in this picture is the minimal solution to the action $S=\int L d t$. In quantum field theories, where the forces and the objects (particles) are represented by quantum fields, the starting point is the Lagrange density, which will from now be called the Lagrangian according to standard nomenclature. To ensure that physical laws can be formulated without a priori knowledge of the reference frame, any realistic Lagrangian must be Lorentz invariant (symmetric under the Lorentz group). In addition, a particular Lagrangian is defined from imposing a constraining internal invariance, which dictate the functional form of the Lagrangian. The internal symmetries imposed on the Standard Model is in part found from experimental data and in part created by the ingenuity of model builders. One major consequence of this is that by giving the particle spectrum with the corresponding masses and imposing a particular internal symmetry on the Lagrangian everything else follows as a necessity, including the forces in nature and in particular, the 


\section{Introduction}

dynamics of particle interactions.

\subsubsection{The Standard Electroweak Theory}

The Standard Electroweak Theory was in the 1960's constructed by Glashow, Salam and Weinberg [2] as a new model of particle interaction that unified the electromagnetic and the weak forces. It is a gauge theory based on the internal symmetries (or gauge groups) $\mathrm{SU}_{L}(2) \times \mathrm{U}_{Y}(1)$, defining a set of transformations on the left-handed (L) fermions and the fermions with hypercharge Y. The fermions are the six leptons and the six quarks, where the leptons are the electron $(e), \operatorname{muon}(\mu)$, tau $(\tau)$ and the corresponding neutrinos $\left(\nu_{e}, \nu_{\mu}\right.$ and $\left.\nu_{\tau}\right)$. The quarks are the up $(u)$, down $(d)$, charm $(c)$, strange $(s)$, top $(t)$ and bottom $(b)$. Associated with the gauge

group generators there are a set of gauge fields $W_{\mu}^{i}(i=1,2,3)$ and $B_{\mu}$ for $\mathrm{SU}(2)$ and U(1) respectively. The fermions are in the fundamental representation of the gauge groups and the gauge fields are in the adjoint representation of the gauge groups, and they transform accordingly [3].

Because of different transformation rules for left-handed and right-handed particles it is a well justified custom to present the particle spectrum in terms of their handedness. Each particle is a four component Dirac spinor $f$ and the left-handed $f^{L}$ and right-handed $f^{R}$ component can be extracted through the two projection 


\subsection{The Standard Model}

$$
\begin{array}{cccccc}
\mathrm{Y}=-1 & \mathrm{Y}=1 / 3 & \mathrm{Y}=-2 & \mathrm{Y}=0 & \mathrm{Y}=-2 / 3 & \mathrm{Y}=4 / 3 \\
\Psi_{e}^{L}=\left[\begin{array}{c}
\nu_{e}^{L} \\
e^{L}
\end{array}\right] & \Psi_{d}^{L}=\left[\begin{array}{l}
u^{L} \\
d^{L}
\end{array}\right] & \psi_{e}^{R}=e^{R} & \psi_{\nu_{e}}^{R}=\nu_{e}^{R} & \psi_{d}^{R}=d^{R} & \psi_{u}^{R}=u^{R} \\
\Psi_{\mu}^{L}=\left[\begin{array}{c}
\nu_{\mu}^{L} \\
\mu^{L}
\end{array}\right] & \Psi_{s}^{L}=\left[\begin{array}{c}
c^{L} \\
s^{L}
\end{array}\right] & \psi_{\mu}^{R}=e^{R} & \psi_{\nu_{\mu}}^{R}=\nu_{\mu}^{R} & \psi_{s}^{R}=s^{R} & \psi_{c}^{R}=c^{R} \\
\Psi_{\tau}^{L}=\left[\begin{array}{c}
\nu_{\tau}^{L} \\
\tau^{L}
\end{array}\right] & \Psi_{b}^{L}=\left[\begin{array}{l}
t^{L} \\
b^{L}
\end{array}\right] & \psi_{\tau}^{R}=\tau^{R} & \psi_{\nu_{\tau}}^{R}=\nu_{\tau}^{R} & \psi_{b}^{R}=b^{R} & \psi_{t}^{R}=t^{R}
\end{array}
$$

Table 1.1: Shown are the left-handed and right-handed fermions in the standard model. The possible right-handed neutrinos are also listed, however, they do not couple to the gauge fields due to zero electroweak quantum numbers.

operators $P_{L}$ and $P_{R}$,

$$
\begin{array}{ll}
f^{L}=P_{L} f, & P_{L}=\frac{1}{2}\left(1-\gamma_{5}\right), \\
f^{R}=P_{R} f, & P_{R}=\frac{1}{2}\left(1+\gamma_{5}\right),
\end{array}
$$

giving $f^{L}+f^{R}=f$. The left-handed particles occur in isospin doublets, justifying the concept of fermion families, while the right-handed particles occur as isospin singlets. For every fermion shown in Table 1.1, there also exist an anti-fermion with opposite quantum number. The hyper charge for each particle is shown above each column and the third component of weak isospin $T_{3}$ is $+1 / 2$ for the upper elements of the isodoublets, and $-1 / 2$ for the for the lower elements. The electric charge $Q$ of each particle is then given by $Q=\frac{Y}{2}+T_{3}$.

In order to generate gauge boson and lepton masses without breaking the invari- 


\section{Introduction}

ance of the Lagrangian and keeping a renormalizable theory the Higgs boson doublet $H$ is introduced [4], which through a non-zero vacuum expectation value

$$
\langle 0|H| 0\rangle=\frac{1}{\sqrt{2}}\left(\begin{array}{l}
0 \\
\nu
\end{array}\right),
$$

spontaneously breaks the $\mathrm{SU}(2) \times \mathrm{U}(1)$ symmetry ${ }^{1}$. The electroweak Lagrangian $\mathcal{L}^{e w}$ is conveniently broken into a set of separate terms where each serve a specific purpose,

$$
\mathcal{L}^{e w}=\mathcal{L}^{F}+\mathcal{L}^{B}+\mathcal{L}^{H}+\mathcal{L}^{F H}
$$

The $\mathcal{L}^{F}$ term gives the kinetic term for the fermions and the coupling between fermions and the gauge bosons. For each left-handed isodoublet $\Psi_{m}^{L}(m=e, \mu, \tau$, $d, s$ and $b)$ and for each right-handed isosinglet $\psi_{n}^{R}\left(n=e, \mu, \tau,\left(\nu_{e}, \nu_{\mu}, \nu_{\tau}\right), d, s, b\right.$, $u, c$ and $t)$,

$$
\mathcal{L}^{F}=i \bar{\psi}_{n}^{R} \gamma^{\mu}\left(\partial_{\mu}+i \frac{g^{\prime}}{2} Y B_{\mu}\right) \psi_{n}^{R}+i \bar{\Psi}_{m}^{L} \gamma^{\mu}\left(\partial_{\mu}+i \frac{g^{\prime}}{2} Y B_{\mu}+i \frac{g}{2}\left[\tau \cdot W_{\mu}\right]\right) \Psi_{m}^{L},(1
$$

where $\tau$ is the SU(2) generators, satisfying the algebra of the Pauli matrices. This term causes the electroweak interaction felt by the fermions and provides a description for how this occurs with the presence of gauge fields. The kinetic terms for the

\footnotetext{
${ }^{1}$ The Higgs doublet has hyper charge $Y=1$.
} 


\subsection{The Standard Model}

gauge fields and the gauge field self-couplings are given by

$$
\begin{aligned}
\mathcal{L}^{B} & =-\frac{1}{4} B_{\mu \nu} B^{\mu \nu}-\frac{1}{4} G_{i \mu \nu} G_{i}^{\mu \nu}, \\
B^{\mu \nu} & =\partial^{\nu} B^{\mu}-\partial^{\mu} B^{\nu}, \\
G_{i}^{\mu \nu} & =\partial^{\nu} W_{i}^{\mu}-\partial^{\mu} W_{i}^{\nu}+g \varepsilon_{i j k} W_{i}^{\mu} W_{i}^{\nu},
\end{aligned}
$$

and allows for interaction among the gauge fields themself. The kinetic term and the self coupling for the Higgs doublet together with the Higgs coupling to the gauge fields are given as

$$
\begin{aligned}
\mathcal{L}^{H} & =\left[D^{\mu} H\right]^{\dagger}\left[D^{\mu} H\right]-\mu^{2} H^{\dagger} H-\lambda\left[H^{\dagger} H\right]^{2}, \\
D^{\mu} H & =\left(\partial^{\mu}+i \frac{g}{2}\left[\tau \cdot W_{\mu}\right]+i \frac{g^{\prime}}{2} B^{\mu}\right) H,
\end{aligned}
$$

and is responsible for generating the mass terms of the gauge fields. This can readily be seen (in the unitary gauge) where the Higgs doublet is expressed as a physical field $\sigma$ (deviation from the vacuum value) plus its vacuum expectation value

$$
H=\frac{1}{\sqrt{2}}\left(\begin{array}{c}
0 \\
\nu+\sigma
\end{array}\right),
$$

with $\nu=\left(-\mu^{2} / \lambda\right)^{1 / 2}$. Fermion mass terms are generated through the Yukawa part of the Lagrangian

$$
\mathcal{L}^{F H}=-x_{l}\left[\bar{\Psi}_{l}^{L} \psi_{l}^{R} H+H^{\dagger} \bar{\psi}_{l}^{R} \Psi_{l}^{L}\right]-X_{l l^{\prime}}\left[\bar{\Psi}_{l}^{L} \psi_{\nu_{l^{\prime}}}^{R} \widetilde{H}+\widetilde{H}^{\dagger} \bar{\psi}_{\nu_{l^{\prime}}}^{R} \Psi_{l}^{L}\right]
$$




\section{Introduction}

$$
\begin{aligned}
& -z_{q}\left[\bar{\Psi}_{q}^{L} \psi_{q}^{R} H+H^{\dagger} \bar{\psi}_{q}^{R} \Psi_{q}^{L}\right]-Z_{q q^{\prime}}\left[\bar{\Psi}_{q}^{L} \psi_{q^{\prime}}^{R} \widetilde{H}+\widetilde{H}^{\dagger} \bar{\psi}_{q^{\prime}}^{R} \Psi_{q}^{L}\right], \\
\widetilde{H}= & -i\left[H^{\dagger} \tau_{2}\right]^{T},
\end{aligned}
$$

with $\left(l, l^{\prime}=e, \mu, \tau\right.$ and $q=d, s, b$, and $\left.q^{\prime}=u, c, t\right)$. The $X$ and $Z$ are $3 \times 3$ Hermitian non diagonal coupling matrices and by diagonalization, one obtains the mass eigenstates for the leptons and the quarks. $x$ and $z$ are kept diagonal without any loss of generality. Originally, the standard electroweak model by Glashow, Salam and Weinberg did not include the quarks, and $X$ was set to zero to force zero neutrino masses. A non-diagonal $Z$ allows for the flavor changing charged current in the quark sector, while a non-diagonal $X$ allows for the neutrino oscillations currently observed [5].

Mass eigenstates found from diagonalizing the gauge boson mass matrices give the physical fields responsible for the various forces. Mediated by the photon, the electromagnetic field is represented by

$$
A^{\mu}=\sin \theta_{w} W_{3}^{\mu}+\cos \theta_{w} B^{\mu}
$$

with zero mass. The weak mixing angle $\theta_{w}$ (or Weinberg angle) is at the tree level given by $\tan \theta_{w}=g^{\prime} / g$. A second neutral mass eigenstate, the $Z$ gauge boson, mediates the neutral weak force

$$
Z^{\mu}=\cos \theta_{w} W_{3}^{\mu}-\sin \theta_{w} B^{\mu},
$$




\subsection{The Standard Model}

with mass $m_{Z}=(g \nu) /\left(2 \cos \theta_{w}\right)$. We further identify the mediators of the charged nuclear weak force

$$
W^{\mu \pm}=\frac{1}{\sqrt{2}}\left(W_{1}^{\mu} \mp i W_{2}^{\mu}\right)
$$

with mass $m_{W}=m_{Z} \cos \theta_{w}$. For the physical neutral Higgs boson the mass is $m_{H}=\sqrt{2 \lambda \nu^{2}}$ and we see that the boson mass spectrum is defined by the four parameters $g, g^{\prime}, \nu, \lambda$. A serious and non trivial test of the model can be obtained by high precision measurements of the above parameters $\left(\sin \theta_{w}, m_{Z}, m_{W}\right)$ and of parameters such as the fine structure constant $\alpha=\left(g g^{\prime}\right)^{2} /\left(4 \pi\left(g^{2}+g^{\prime 2}\right)\right)$ and the Fermi coupling constant $G_{F}=1 /\left(\sqrt{2} \nu^{2}\right)$. So far the SM has passed this test with bravo, with a precision of $1 \times 10^{-3}, 8 \times 10^{-5}, 1 \times 10^{-3}, 5 \times 10^{-8}$ and $1 \times 10^{-4}$ in $\sin ^{2} \theta_{w}$, $m_{Z}, m_{W}, \alpha$ and $G_{F}$ respectively ${ }^{2}$. This is the remarkable legacy of the electroweak standard model.

Of particular interest for this analysis is the non-diagonal mass matrix $Z$ in equation (1.8). The diagonal mass matrix $Z\left(V Z V^{\dagger}=\right.$ diagonal) will give the quark mass eigenstates (or physical states) $q^{\prime}$. Expressed as linear combinations of the original quark fields $q$ listed in Table 1.1 , the physical fields $q^{\prime}$ are $\left(u^{\prime}, c^{\prime}, t^{\prime}\right)=(u, c, t)$ and $\left(d^{\prime}, s^{\prime}, b^{\prime}\right)=V(d, s, b)$. This relation is expressed through the CKM matrix $V$

\footnotetext{
${ }^{2}$ The expressions given for the various parameters are the tree level results. Higher order corrections normally modifies these expressions to also include the mass of the top quark (often quadratic dependent) and the mass of the Higgs boson (logarithmic dependent).
} 


\section{Introduction}

which in the parameterization of Wolfenstein [7] reads

$$
V=\left(\begin{array}{ccc}
1-\frac{\lambda^{2}}{2} & \lambda & \lambda^{3} A(\rho-i \eta) \\
-\lambda & 1-\frac{\lambda^{2}}{2} & \lambda^{2} A \\
\lambda^{3} A(1-\rho-i \eta) & -\lambda^{2} A & 1
\end{array}\right)
$$

This relationship between quark gauge fields and physical quark fields gives rise to the previously mentioned flavor changing charged currents. This can be seen by first writing the weak charged current Lagrangian $\mathcal{L}_{c c}$ in terms of the physical charged gauge bosons $W^{ \pm}$

$$
\mathcal{L}_{c c}=-\bar{\Psi}_{m}^{L} \gamma^{\mu} \frac{g}{2}\left[\tau_{1} W_{\mu}^{1}+\tau_{2} W_{\mu}^{2}\right] \Psi_{m}^{L}=-\frac{g}{\sqrt{2}} \bar{\Psi}_{m}^{L} \gamma^{\mu}\left(\begin{array}{cc}
0 & W_{\mu}^{+} \\
W_{\mu}^{-} & 0
\end{array}\right) \Psi_{m}^{L},
$$

and by summing over the indices $m=d, s, b$ it can be written explicitly in terms of the quark fields $q$,

$$
\mathcal{L}_{c c}=-\frac{g}{\sqrt{2}}\left(\bar{u}^{L}, \bar{c}^{L}, \bar{t}^{L}\right) \gamma^{\mu} W_{\mu}^{+}\left(\begin{array}{c}
d^{L} \\
s^{L} \\
b^{L}
\end{array}\right)+\text { h.c. },
$$

and finally, in terms of the physical quark fields $q^{\prime}$,

$$
\mathcal{L}_{c c}=-\frac{g}{\sqrt{2}}\left(\bar{u}^{\prime}, \bar{c}^{L}, \bar{t}^{L}\right) \gamma^{\mu} W_{\mu}^{+} V^{\dagger}\left(\begin{array}{c}
d^{L} \\
s^{L} \\
b^{L}
\end{array}\right)+\text { h.c. }
$$




\subsection{The Standard Model}

Because of the ordering of the CKM matrix $V$, the transition of a $b$ quark into a $c$ quark is proportional to $A^{2} \lambda^{4}(\sim 0.002)$, while the transition of a $b$ quark into a $u$ quark is proportional to $A^{2} \lambda^{6}\left(\rho^{2}+\eta^{2}\right)(<0.00002)$. Hence, the charmless decay $b \rightarrow u$ is strongly suppressed, by approximately a factor of 100 , compared to the favorable charm decay $b \rightarrow c$. Naturally then, charmless $b$ decays are often been quoted as rare $b$ decays, and it is just recently that such modes have been observed in the special case where no leptons are in the final state [8].

\subsubsection{Quantum Chromodynamics}

The physics of the strong interaction developed in an explosive manner during the seventies. The peak of this development came with the construction of quantum chromodynamics (QCD), which lays claim to be the fundamental theory of this area of physics. The strong interaction describes forces that only act between hadrons, and are not felt by leptons.

Historically, QCD originated as a development of the quark model. Gell-Mann and Zweig (1964) postulated that physical hadrons are composite objects, made up of three quarks (baryons) or of a quark-antiquark pair (mesons). The three quarks flavors are what we now call the up (u), down (d) and strange (s) which was in the original theory contained in the fundamental representation of flavor $\mathrm{SU}_{F}(3)$. 


\section{Introduction}

It has been found that those representations of $\mathrm{SU}_{F}(3)$ occur that may be found by reducing the products $3 \times 3 \times 3$ (baryons) or $3 \times \overline{3}$ (mesons). That hadrons are composite objects was a welcome hypothesis on other grounds too. The proton magnetic moment is $\mu_{p}=2.79 e / 2 m_{p}$, instead of $\mu_{p}=e / 2 m_{p}$ expected if the proton was elementary. This way of understanding the hadronic particle spectrum was first considered merely as an accounting technique, but because of its success it triggered a massive search for quarks that still goes on. A quite different form of evidence for hadron constituents was found around 1969 at Stanford in the electron-nucleon scattering experiment $e+N \rightarrow e+X$. The large deflection angle of the electrons was a clear indication that nucleons can be considered as bound states of certain constituents. This is the same kind of argument that was used by Rutherford when he observed the scattering of $\alpha$-particles from atoms, and concluded that atoms are made up of smaller constituents.

The Gell-Mann picture can be challenged, however, for its lack of ability to explain the $\Delta^{++}$resonance, which is understood as a bound state of three up quarks with their spin aligned. Being a fermion the quark should obey the Fermi-Dirac statistics and their state should be antisymmetric. However, by introducing a new internal quantum number which Gell-Mann and his collaborators called color, each flavor of quark may come in any of three colors $\mathrm{i}=\mathrm{r}, \mathrm{y}, \mathrm{v}$ (red,yellow,violet). One can 


\subsection{The Standard Model}

then reinterpret $\Delta^{++}$as the antisymmetric $\epsilon^{i j k} u_{i} u_{j} u_{k}$ with the quark spins still being aligned. By essentially tripling the quark count, the introduction of color further explained the large cross section of $e^{+} e^{-} \rightarrow$ hadrons as compared to the similar cross section into leptons. Further, in order to construct the strong interaction in a similar way as was done for the electroweak theory, Weinberg and Nanopoulos (1973) have shown that, to avoid catastrophic violations of parity to order $\alpha_{s}$, one needs strong interactions to act on quantum numbers other than flavor. These were among the reasons that led physicists to consider the possibility that whatever glued the quarks together, interacted equally on color to which weak and electro magnetic interaction are blind.

The theory of QCD is a SU(3) color gauge theory where each quark flavor comes with three colors in the fundamental representation of $\mathrm{SU}_{c}(3)$. There are eight vector gluons $B_{a}^{\mu}$, a $=1, \ldots, 8$, in the adjoint representation of $\mathrm{SU}_{c}(3)$, which interact universally with all quark flavors

$$
\mathcal{L}_{\text {int }}^{Q C D}=g_{s} \bar{Q}_{q} \gamma_{\mu}\left[t \cdot B^{\mu}\right] Q_{q},
$$

where $t$ is the eight generators, $t^{a}=\lambda^{a} / 2$ with $\lambda$ being the Gell-Mann matrices. The $q$ is the flavor index (up, down, charm, strange, top and bottom) and the quark field 


\section{Introduction}

$Q$ is the $\mathrm{SU}(3)$ color triplet

$$
Q_{q}=\left[\begin{array}{c}
q_{\text {red }} \\
q_{\text {yellow }} \\
q_{\text {violet }}
\end{array}\right] .
$$

The gluon self interaction is given by

$$
\begin{aligned}
\mathcal{L}_{B}^{Q C D} & =-\frac{1}{4} G_{a}^{\mu \nu} G_{a \mu \nu}, \\
G_{a}^{\mu \nu} & =\partial^{\mu} B_{a}^{\nu}-\partial^{\nu} B_{a}^{\mu}+g_{s} f_{a}^{b c} B_{b}^{\mu} B_{c}^{\nu}, \\
f^{a b c} t^{c} & =-i\left[t^{a}, t^{b}\right],
\end{aligned}
$$

and is responsible for the interaction among gluons themself. With the kinetic terms and the mass terms for the quarks already expressed in the previous section, the QCD Lagrangian is simply

$$
\mathcal{L}^{Q C D}=\mathcal{L}_{\text {int }}^{Q C D}+\mathcal{L}_{B}^{Q C D}
$$

and is a description for the strong interaction where the massless gluons are the mediator for the responsive quarks. A nice feature of QCD is that in such a theory (a massless Yang Mills theory with not too many flavors participating) the effective coupling constant $g_{s}$ decreases at short distances (high energy) and increases at long distances (low energy). Figure 1.1 shows the coupling constant scale dependence. 


\subsection{The Standard Model}

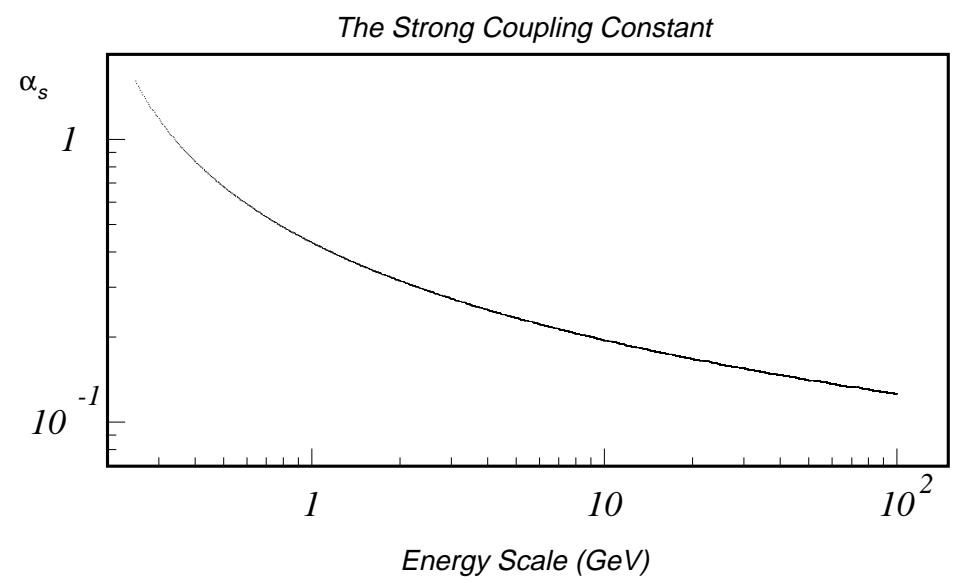

Figure 1.1: The strong coupling constant $\alpha_{s}=g_{s}^{2} / 4 \pi[9]$ evolves with the energy at which the interaction takes place.

This feature of the strong coupling constant makes QCD a remarkable theory in that it can confine colored particles into color singlet hadrons (hadronization) on a scale $<1 \mathrm{GeV}$, and accounts for why particularly strong interactions occur on this scale. This prevents the liberation of individual quarks and gluons and explains why free quarks and gluons have not been observed directly. At the same time, it can provide interactions that become weak if short distances are probed. A quark struck at high energy behaves as if it is essentially free.

At short distances with a small $g_{s}$, QCD has been shown to be valid as a perturbation theory while at long distances with a quite large $g_{s}$ other methods must be 


\section{Introduction}

employed to extract information from the theory. This is somewhat unfortunate and can cause large theoretical uncertainties in certain processes due to non-perturbative QCD contributions, as will be illustrated later.

\section{$1.2 e^{+} e^{-}$Collisions}

Particle interactions are conveniently expressed in terms of Feynman diagrams, both for visual clarity and for book keeping purposes. The cross section or rate for a particular process is essentially the square of the sum over all different topological diagrams which in the end cause the same outcome. The production of two fermions $f \bar{f}$ can be understood as the annihilation of the $e^{+} e^{-}$initial particles into a $\gamma$ or a $Z$ with a subsequent decay of the $\gamma$ or the $Z$ boson (Figure 1.2). For each vertex (the intersection of three particle lines) there is a corresponding coupling constant $\alpha$. For electroweak interactions this coupling constant is rather small $\left(\mathcal{O}\left(10^{-2}\right)\right)$ causing higher order diagrams (in $\alpha$ ) to be less significant and the production of lepton pairs

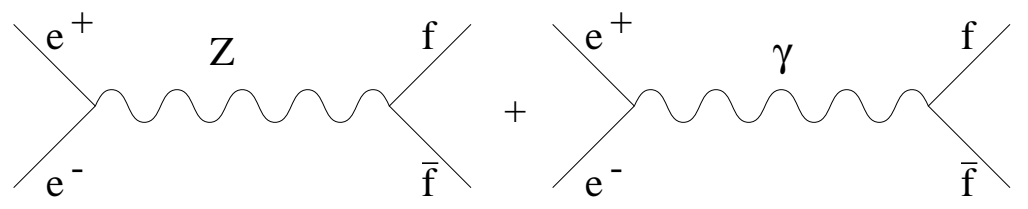

Figure 1.2: The tree level Feynman diagrams for the production of a fermion anti-fermion pair through $\gamma$ and $Z$ exchange. 

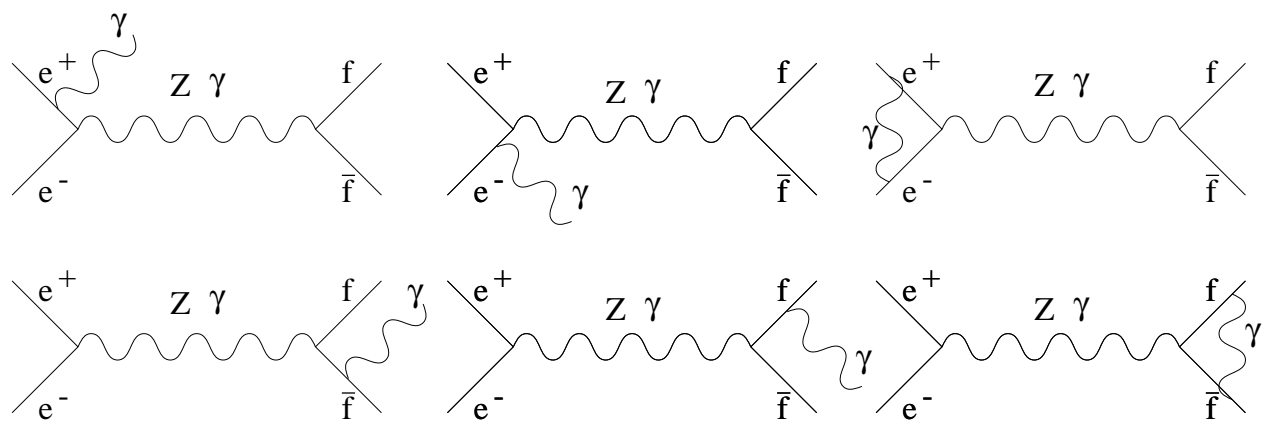

Figure 1.3: Leading-order QED initial-state (first line) and final-state (second line) radiative corrections to the $e^{+} e^{-} \rightarrow f \bar{f}$ process. The vertex correction diagrams are here included since they cancel infrared divergences in the photon emission diagrams.

can therefore be calculated to large precision by including the contributions from only a few diagrams. Figure 1.3 shows a set of diagrams that must be considered if the next order in $\alpha$ is to be calculated for $e^{+} e^{-} \rightarrow f \bar{f}$. Emission of a photon does not directly give the same outcome, still, they must be included due to the undetectable soft photons which causes the process to behave, in an experimental sense, as the ones without a photon emission. The initial state correction has a rather large effect at the $Z$ pole since it lowers the energy of the initial leptons and moves the center of mass energy away from the optimal energy for producing the $Z$. The effect is a decrease of the peak cross section by $29 \%$ [10]. With the inclusion of second order diagrams this correction is known to a relative precision of $0.2 \%[11]$. Final state radiation has a much smaller effect, $0.02-0.08 \%$. While the 


\section{Introduction}
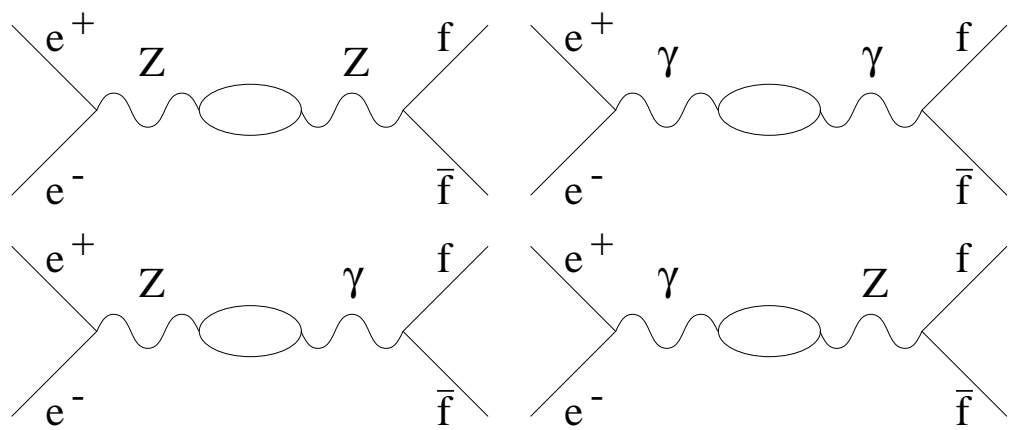

Figure 1.4: Oblique corrections to the $e^{+} e^{-} \rightarrow f \bar{f}$ process.

initial-state QED radiative corrections have a large effect on precision electroweak measurements, the virtual propagator and vertex corrections are more interesting in that these loop diagrams renormalize the measured electroweak parameters. In particular, the electroweak propagator corrections provide information on the still unknown Higgs mass through the interaction of this scalar with the gauge bosons. The type of diagrams that are responsible for the propagator corrections (or oblique corrections) are shown in figure 1.4. The loops represents the vacuum self energy of the bosons where the propagators split into a fermion anti-fermion pair (or a Higgs pair) which then recombine again to a boson. For more electroweak diagrams and a complete review of first order corrections see [13] and references therein.

The production of lepton pairs is fully explained by the set of electroweak diagrams and the experimental situation is well understood. When a $Z$ decays into 


\section{$1.2 e^{+} e^{-}$Collisions}
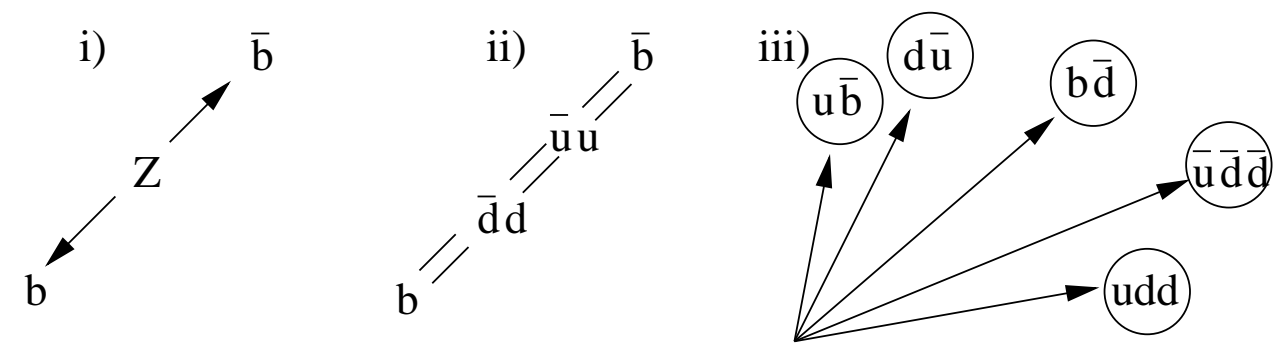

Figure 1.5: A simple interpretation of quark hadronization is shown. i) The $Z$ decays into a quark anti-quark pair $(b \bar{b})$. ii) As the strong field stretches between the two outgoing energetic quarks, new quark pairs are created out of vacuum. iii) This process continues until the shower has cooled off enough and hadrons are created.

a lepton pair (excluding the neutrinos), the two leptons travel outward opposite each other and interact with the detector and/or decay. When a $Z$ decays into two neutrinos, they pass through the detector and remain unseen. However, when a $Z$ decays into a quark anti-quark pair (for example $Z \rightarrow b \bar{b}$ ), something more complex happens. Although the strict process of $Z \rightarrow q \bar{q}$ can be calculated from the electroweak diagrams together with QCD corrections [14], it is not a realistic picture of reality. It is an experimental fact that quarks are always observed together with one anti-quark or two other quarks in particles called hadrons, and never isolated by themselves. QCD explains this effect through the confinement of gluons and quarks. When a quark is given enough energy to escape the attraction of one or more other quarks, much of its energy is absorbed in a QCD field, consisting of gluons, which are subsequently converted into one or more quark anti-quark pairs. Eventually, one or 


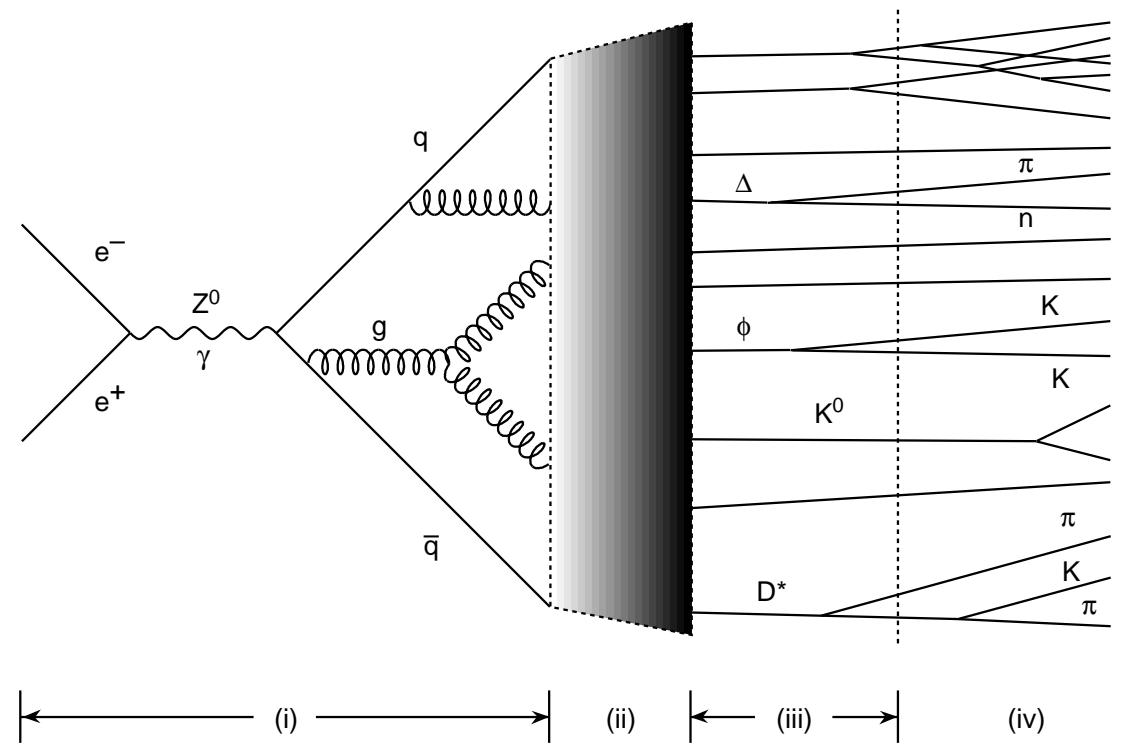

Figure 1.6: The various stages of hadron production are shown.

more of these new quarks remain with the escaping quark, producing a new hadron in which to confine the quark. Any, remaining quarks may produce several other, lower energy, hadrons. This process which is called hadronization is illustrated in Figure 1.5. Figure 1.6 shows the conventional view of the reaction $e^{+} e^{-} \rightarrow$ hadrons.

- i) - Parton Shower Formation. This stage is understood theoretically as the creation of a quark anti-quark pair and the initiation of the parton shower, calculable in perturbative QCD down to the level of $\alpha_{s} \simeq 1$ [14]. The lowest order $e^{+} e^{-} \rightarrow q \bar{q}$ (Figure 1.2) which is calculable from electroweak diagrams is modified to first order in QCD by the emission of a gluon. 


\section{$1.2 e^{+} e^{-}$Collisions}

- ii) - Hadronization. In this phase the partons (quarks or gluons) produced in phase i) have reached the hadron mass energy scale (confinement scale), and cannot fragment further and form hadrons. Due to the non-perturbative nature of the process, several phenomenological models have been proposed to describe it. The String Fragmentation model is implemented in JETSET [64] which is used for generating hadronic MC events at SLD.

- iii) - Decay of Unstable Hadrons. In this phase the heavy and unstable hadrons produced in phase ii) such as $D$ and $B$ mesons decay into the experimentally observable hadrons such as pions and kaons, phase iv). Again, perturbative QCD cannot be used here and the experimentally measured branching ratios (if measured) are used when models are constructed. For certain weak decays, one can attempt to use electroweak theory as a starting point but eventually phenomenological descriptions become necessary here as well.

From the above picture it is clear that the theoretical calculation of a particular process is difficult to do from the first principle in QCD. Hence, the production rates of $B$ mesons, which are of essential importance in this analysis, are extracted from measurements. On the other hand, the calculation of the cross section of $e^{+} e^{-}$into all hadrons can successfully be calculated from perturbative QCD. This is possible 
Introduction

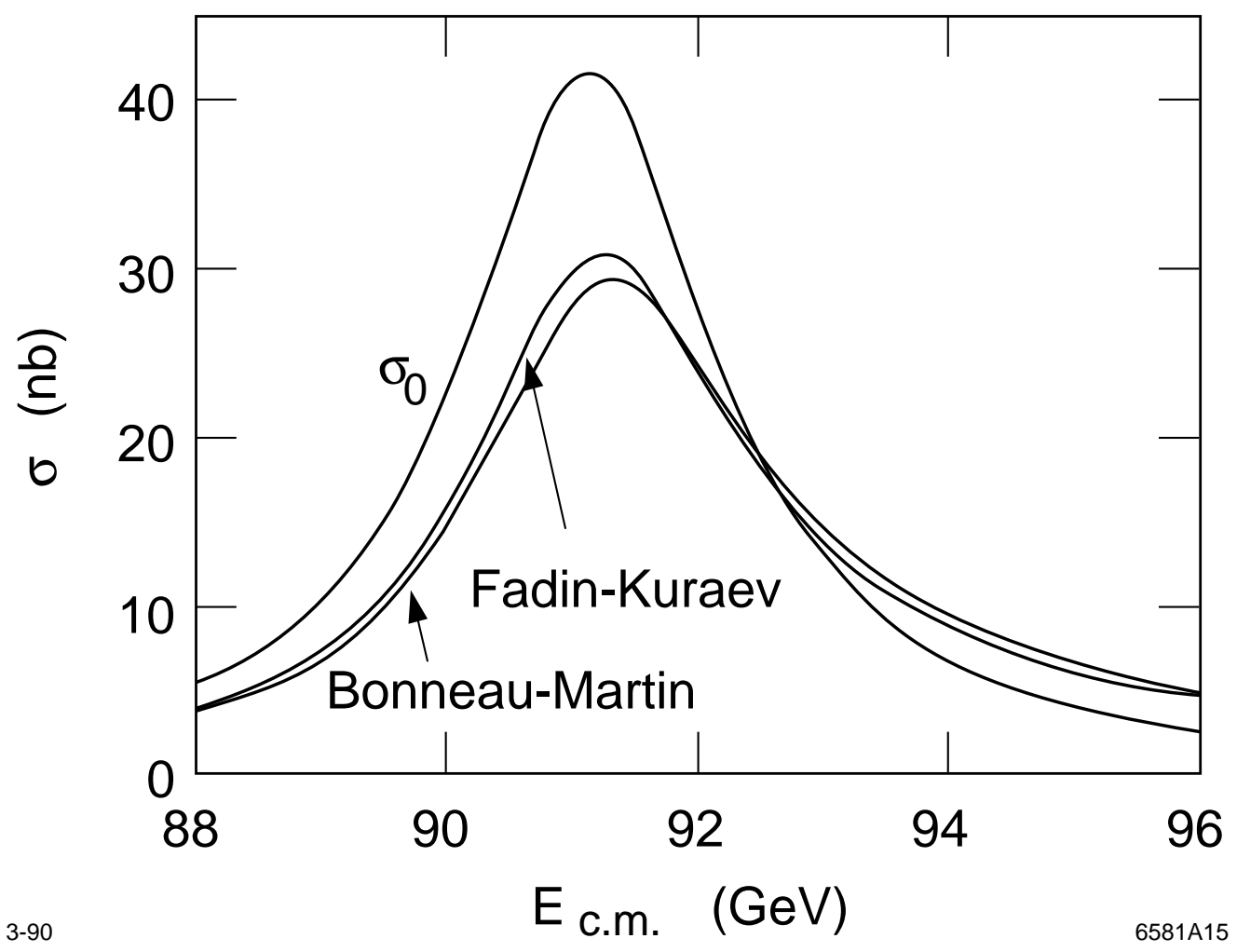

Figure 1.7: The hadronic cross section is shown for $e^{+} e^{-}$annihilation.

due to the simplification that the sum over all individual hadronic states $|h\rangle$ must equal one, $\sum|h\rangle\langle h|=1$. See [9] for further details. Figure 1.7 shows the all hadronic cross section in $e^{+} e^{-}$annihilation through the $Z$ exchange. $\sigma_{0}$ is the $Z$-pole result, which excludes initial state corrections. Also shown are the cross sections when first order initial state radiation (Bonneau-Martin [10]) and second order initial state radiation (Fadin-Kuraev [11]) are included. Also, the QCD corrections are taken 


\begin{tabular}{cc|cc}
$E_{\mathrm{cm}}(\mathrm{GeV})$ & $\sigma_{\text {had }}(\mathrm{nb})$ & $E_{\mathrm{cm}}(\mathrm{GeV})$ & $\sigma_{\text {had }}(\mathrm{nb})$ \\
\hline 91.17 & 30.32 & 91.25 & 30.51 \\
91.18 & 30.36 & 91.26 & 30.52 \\
$m_{Z}$ & 30.38 & 91.27 & 30.52 \\
91.20 & 30.42 & 91.28 & 30.52 \\
91.21 & 30.44 & 91.29 & 30.52 \\
91.22 & 30.46 & 91.30 & 30.52 \\
91.23 & 30.48 & 91.31 & 30.51 \\
91.24 & 30.50 & 91.32 & 30.50 \\
\hline
\end{tabular}

Table 1.2: The hadronic cross-section in $e^{+} e^{-}$collisions are shown for a set of center of mass energies.

into account and have the effect of increasing the cross-section by around $4 \%$ [12]. Table 1.2 gives the hadronic cross-section for a selected set of energies as calculated with ZFITTER version 5.21 (March 1999) [15]. The Higgs mass is taken to be 100 $\mathrm{GeV}$, the top mass is set to $173.8 \mathrm{GeV}$ while a $Z$ mass of $91.1867 \mathrm{GeV}$ is used. The strong coupling constant at the $Z$ pole is 0.119 .

To estimate the theoretical uncertainty of the hadronic cross-section it is useful to note that the SM prediction for the $Z$-pole cross-section $\sigma_{0}$ is given in the PDG [1] as $41.469 \pm 0.016$. By including the uncertainty of the initial state radiative corrections $(0.2 \%)$ the uncertainty of the hadronic cross-section $\sigma_{\text {had }}$ becomes \pm 0.03 . SLC has been running at the average center of mass energy $91.28 \mathrm{GeV}$ and by including an uncertainty of $40 \mathrm{MeV}$ on this number the hadronic cross-section that is used in this 


\section{Introduction}

\begin{tabular}{c|rc} 
& \multicolumn{3}{|c}{ VXD2 } & VXD3 \\
\hline$B^{+}$or $B^{-}$ & $28781 \pm 1491$ & $73433 \pm 3803$ \\
$B^{0}$ or $\bar{B}^{0}$ & $28781 \pm 1491$ & $73433 \pm 3803$ \\
$B_{s}$ or $\bar{B}_{s}$ & $7612 \pm 1453$ & $19422 \pm 3707$
\end{tabular}

Table 1.3: The number of $B$ mesons produced at SLD.

analysis is

$$
\sigma_{\text {had }}=(30.52 \pm 0.04) \mathrm{nb}
$$

Table 3.1 in Chapter 3 will list the SLC luminosity numbers which together with the hadronic cross-section give the following numbers for the hadronic events at SLD:

$$
\begin{aligned}
& \text { VXD2 }(1993-95): \quad 167121 \pm 1693, \\
& \text { VXD3 }(1996-98): \quad 426395 \pm 4309,
\end{aligned}
$$

where the uncertainties are dominated by the luminosity uncertainties in Table 3.1 . The SLD $B$ meson sample size is calculated from a $(0.2169 \pm 0.0014)$ [1] fraction of $b \bar{b}$ 's in the hadronic sample and a branching ratio of $b \rightarrow B$ meson of $0.397_{-0.022}^{+0.018}$ and $0.105_{-0.022}^{+0.018}$ for $B^{+}$or $B^{0}$ and $B_{s}$ respectively ${ }^{3}$ [1]. Table 1.3 lists the $B$ meson production at SLD, and the uncertainties are dominated by the branching ratio uncertainties of $b \rightarrow B$. These numbers will be of fundamental importance when the results of this analysis will be presented in Chapter 6 .

\footnotetext{
${ }^{3}$ The quark content of the $B$ mesons will be listed in Table 1.5.
} 


\section{$1.3 \quad$ Rare $B$ Decays}

\section{$1.3 \quad$ Rare $B$ Decays}

In recent years considerable attention has been given to charmless $B$ decays. This interest, both at the experimental and at the theoretical level, has been fueled by increased statistics at various experiments, making it possible to achieve experimental sensitivity to match the theoretical predictions for such modes. The total branching ratio of $b$ into charmless final states is expected to be a few percent in the Standard model, with most of the individual modes contributing a few times $10^{-4}$ or less [16]. There are three classes of charmless $B$ decays, where the first two, the radiative type and the dineutrino type, are made possible through the cutely named penguin diagrams. Figure 1.8 shows the first order diagrams responsible for such decays. The third class is named hadronic charmless $B$ decays and contain all

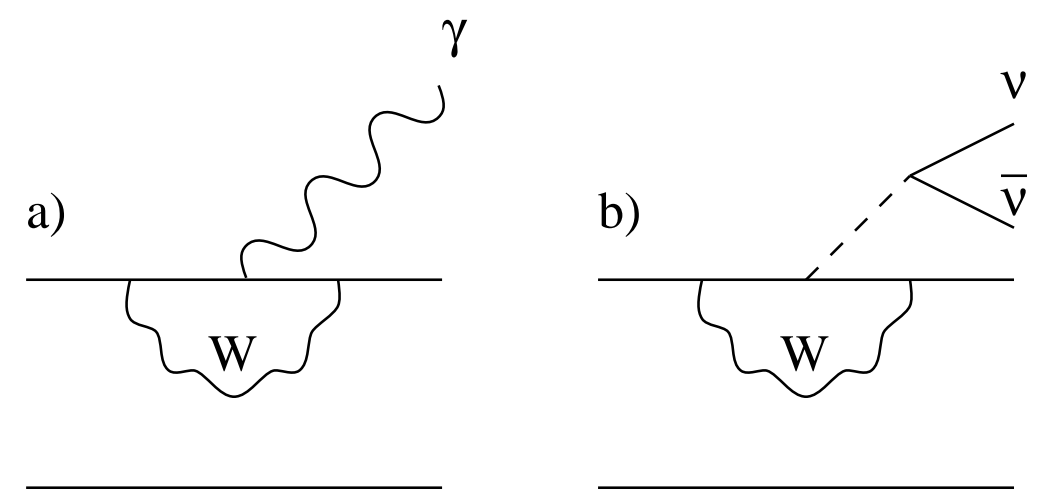

Figure 1.8: Penguin processes are responsible for the a) radiative decays and the $b$ ) dineutrino decays. 


\section{Introduction}

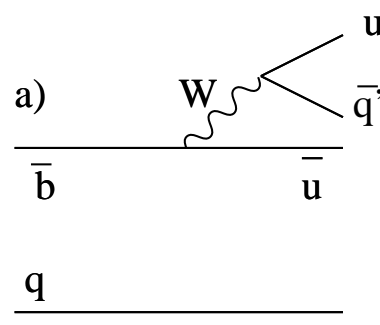

b)
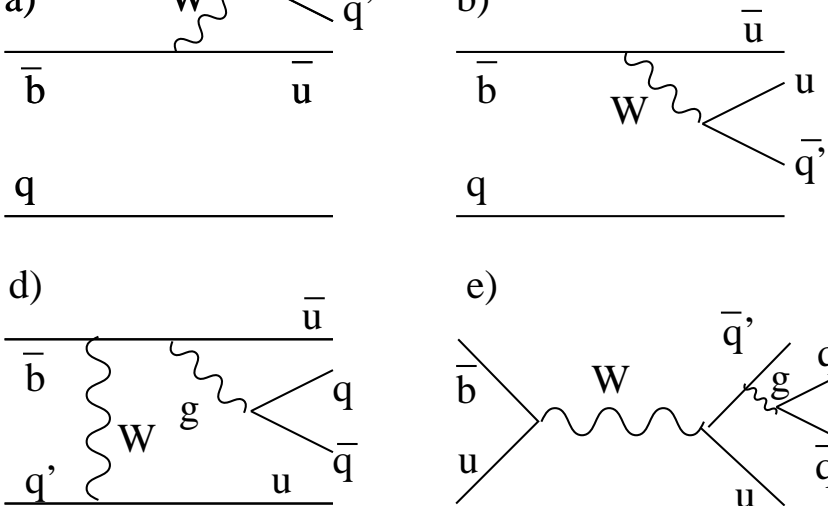

e)

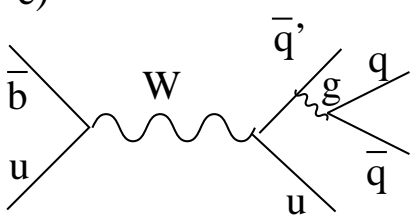

c)
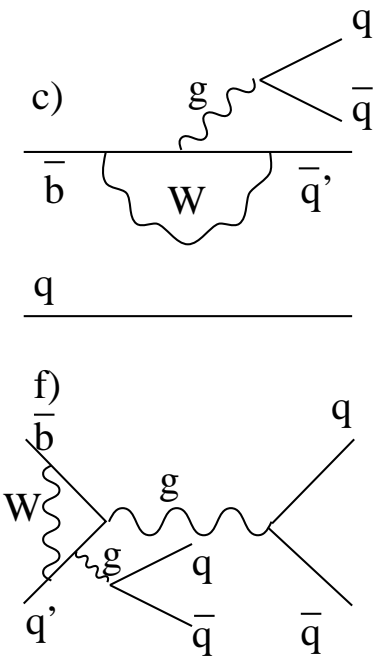

Figure 1.9: Diagrams describing hadronic charmless $B$ decays. The quark $q$ denotes any of the light flavors up (u), down (d) or strange (s), whereas the quark $q^{\prime}$ denotes down or strange.

processes where

$$
B \rightarrow \text { No Open Charm, No Leptons, No Photons. }
$$

Figure 1.9 shows the diagrams at the lowest order that are responsible for the hadronic charmless decays. In all diagrams the quark $q$ denotes any of the light flavors up (u), down (d) or strange (s), whereas the quark $q^{\prime}$ denotes down or strange. Diagram a) represents a color-favored and CKM suppressed tree amplitude, associated with the transition $\bar{b} \rightarrow \bar{q}^{\prime} \bar{u} u$ in which the $\bar{q}^{\prime} u$ system forms a color-singlet pseudoscalar meson $P$ or vector meson $V$ while the $\bar{u}$ combines with the spectator quark $q$ to form the other pseudoscalar meson or vector meson. Diagram b) repre- 


\section{$1.3 \quad$ Rare $B$ Decays}

sents a color-suppressed and CKM suppressed tree amplitude, associated with the transition $\bar{b} \rightarrow \bar{q}^{\prime} \bar{u} u$ in which the $\bar{u} u$ system is incorporated into a neutral pseudoscalar meson or vector meson, while the $\bar{q}^{\prime}$ combines with the spectator quark $q$ to form another meson. Diagram $\mathrm{c}$ ) is a penguin diagram associated with the transition $\bar{b} \rightarrow \bar{q}^{\prime}$ involving virtual quarks of charge $2 / 3$ coupling with one or more gluons in a loop (only one gluon is shown in this diagram). Diagram d) represents an exchange amplitude in which the $\bar{b}$ quark and the the initial $q^{\prime}$ quark from the neutral $B$ meson exchange a $W$ and become a $\bar{u} u$ pair and subsequently a quark pair $\bar{q} q$ is created from vacuum (illustrated by a gluon emission from the $\bar{u}$ quark). An annihilation diagram is pictured in e) and contributes to charged $B$ decays through the subprocess $\bar{b} u \rightarrow u \bar{q}^{\prime}$ by means of a $W$ in the direct channel and subsequently a quark pair $\bar{q} q$ is created from vacuum (illustrated by a gluon emission from the $\bar{q}^{\prime}$ quark). The last diagram, f), is a penguin-annihilation diagram in which an initial neutral $B$ meson $\left(\bar{b} q^{\prime}\right)$ state annihilates into vacuum quantum numbers and two quark pairs are created from vacuum (illustrated to first order as two gluon emissions from two quark lines).

All the six topologically different diagrams shown are the lowest order processes responsible for rare decays, and are calculable from electroweak and QCD perturbative theory. However, the existence of virtual gluons in loop structures causes the 


\section{Introduction}

QCD contributions to be intrinsically non-perturbative which has caused a great deal of suggestive literature for how to properly understand these decays.

Depending on the particular process in question the various diagrams will contribute with varying weight. For example $B^{0} \rightarrow \pi^{+} \pi^{-}$will get contributions from all the diagrams except diagram e) while $B^{0} \rightarrow K^{+} K^{-}$is not possible by the tree level diagrams a)-b) and the annihilation diagram e). However, it is believed in general that the tree diagrams and the penguin diagrams will dominate most processes and that the other diagrams d)-f) will contribute with approximately 5\% [17]. An exception to this is the decays of $B^{0} \rightarrow K^{+} K^{-}, \phi \phi$ or $B_{s} \rightarrow \pi^{+} \pi^{-}, \rho \rho$ which cannot be mediated by the diagrams a),b) and c).

The relative weight of the tree level diagrams versus the penguin diagram is of great importance in understanding loop structures and QCD contributions to various processes. For example, particularly clean modes for the study of $\mathrm{CP}$ violation at the emerging $B$-factories are the 2 body charmless $\mathrm{B}$ decays $B^{0} \rightarrow \pi^{+} \pi^{-}, \rho^{+} \rho^{-}$and a question of great importance, in order to compare theory with soon to come data, is the penguin contributions for these modes [18]. Further, the penguin diagrams play an essential role in the kaon system and in the KM model it is responsible for the direct $\mathrm{CP}$ violation. In spite of its importance, the penguin contribution in $K$ decay is still under intense study and debate [19]. For certain modes (e.g. $B^{ \pm} \rightarrow$ 


\subsection{Rare $B$ Decays}

$\omega K^{ \pm}$), particularly large measured branching ratios cannot be readily explained with the current theoretical framework [20], and causes a great challenge for the theory. Another interesting issue is the result from CLEO and ARGUS on the charm multiplicity $\left(\eta_{c}\right)$ and the semi-leptonic branching ratio $\left(B r_{s l}\right)$ in $B$ decays. The measurements are low in both $\eta_{c}$ and $B r_{s l}$ by around $20 \%$ compared to the preferred Standard Model prediction [21]. A possible solution to this discrepancy is given in [22], where the creation of hybrid charmoniums $\left(\psi_{c} \sim \bar{c} c g\right)$ results in a rapid decay into light hadrons, perhaps enhanced by glueballs, and hence change the signature of the decay to mimic a charmless $B$ decay.

Therefore, the measurement of two-body charmless $B$ decays can provide, in particular, a unique and excellent test of the penguin mechanism and shed some light on QCD calculations. In general, it can become a test ground for the validity of the Standard Model.

\subsubsection{The Search Modes at SLD}

At SLD the rare $B$ decay search is focused on charmless exclusive-hadronic two body decays of $B^{0}, B^{+}$and $B_{s}$ mesons. Table 1.4 lists the search modes in question. The decay products are the charged pseudoscalars $P^{+}($spin 0$)$ and/or the neutral vector particles $V^{0}$ (spin 1$)$. Such two body $B$ decays carry a particular kinematic 


\section{Introduction}

\begin{tabular}{llll} 
Mode & Mode & Mode & Mode \\
& & & \\
\hline$B_{s}$ or $B^{0} \rightarrow P^{+} P^{-}$ & $B^{+} \rightarrow P^{+} V^{0}$ & $B^{0} \rightarrow V^{0} V^{0}$ & $B_{s} \rightarrow V^{0} V^{0}$ \\
& & & \\
\hline$B^{0} \rightarrow \pi^{+} \pi^{-}$ & $B^{+} \rightarrow \rho^{0} \pi^{+}$ & $B^{0} \rightarrow \rho^{0} \rho^{0}$ & $B_{s} \rightarrow \rho^{0} \rho^{0}$ \\
$B^{0} \rightarrow K^{-} \pi^{+}$ & $B^{+} \rightarrow \rho^{0} K^{+}$ & $B^{0} \rightarrow \bar{K}^{* 0} \rho^{0}$ & $B_{s} \rightarrow \bar{K}^{* 0} \rho^{0}$ \\
$B^{0} \rightarrow K^{+} K^{-}$ & $B^{+} \rightarrow K^{* 0} \pi^{+}$ & $B^{0} \rightarrow \bar{K}^{* 0} K^{* 0}$ & $B_{s} \rightarrow \bar{K}^{* 0} K^{* 0}$ \\
$B_{s} \rightarrow \pi^{+} \pi^{-}$ & $B^{+} \rightarrow K^{* 0} K^{+}$ & $B^{0} \rightarrow \phi \rho^{0}$ & $B_{s} \rightarrow \phi \rho^{0}$ \\
$B_{s} \rightarrow K^{+} \pi^{-}$ & $B^{+} \rightarrow \phi \pi^{+}$ & $B^{0} \rightarrow \phi \bar{K}^{* 0}$ & $B_{s} \rightarrow \phi K^{* 0}$ \\
$B_{s} \rightarrow K^{+} K^{-}$ & $B^{+} \rightarrow \phi K^{+}$ & $B^{0} \rightarrow \phi \phi$ & $B_{s} \rightarrow \phi \phi$
\end{tabular}

Table 1.4: Listed are the two body decay modes which is searched for in this analysis. The $B$ decay into two pseudoscalar particles are listed as $B \rightarrow P P$, the decay into a pseudoscalar and a vector particle is listed as $B \rightarrow P V$ and the decay into two vector particles are listed as $B \rightarrow V V$. For each mode listed there also exist an anti mode involving the anti particles.

signature which is exploited in many ways in this analysis (chapter 5). The resonance modes $B^{+} \rightarrow P^{+} V$ and $B^{0}, B_{s} \rightarrow V^{0} V^{0}$ ends up with three or four final pseudoscalars $P$ through the subsequent decay of the vector meson $V$ into two pseudoscalars. This vector decay is governed by the strong force and causes a very rapid decay in such a way that these vectors are not seen directly by the detector, but are identified by the decay products ${ }^{4}$. Hence, the reconstruction of the $B$ mesons can be treated as a three or four prong decay for the $B \rightarrow P V$ and $B \rightarrow V V$ respectively, with kaons and pions being the measured final tracks. The decay table of the vector mesons is listed in PDG [1], and $\rho^{0}$ decays into $\pi^{+} \pi^{-} 100 \%$ of the time, $K^{* 0}$ decays

\footnotetext{
${ }^{4}$ The lifetime of the charged pions $\pi^{ \pm}$and kaons $K^{ \pm}$is around $10^{-8}$ seconds while the lifetimes for the vectors $\rho^{0}, K^{* 0}$ and $\phi$ are $10^{-24}, 10^{-23}$ and $10^{-22}$ seconds respectively.
} 


\subsection{Rare $B$ Decays}

\begin{tabular}{|cc|ll|ll|ll|}
\multicolumn{3}{|c|}{ Decay Products } & \multicolumn{4}{|c|}{ Decay Particles } \\
\hline$\pi^{+}$ & $u \bar{d}$ & $\rho$ & $u \bar{u}, d \bar{d}$ & $B^{0}$ & $d \bar{b}$ & $B^{+}$ & $u \bar{b}$ \\
$\pi^{-}$ & $\bar{u} d$ & $K^{* 0}$ & $d \bar{s}$ & $\bar{B}^{0}$ & $\bar{d} b$ & $B^{-}$ & $\bar{u} b$ \\
$K^{+}$ & $u \bar{s}$ & $\bar{K}^{* 0}$ & $\bar{d} s$ & $B_{s}$ & $s \bar{b}$ & & \\
$K^{-}$ & $\bar{u} s$ & $\phi$ & $s \bar{s}$ & $\bar{B}_{s}$ & $\bar{s} b$ & & \\
& & & & & & & \\
\hline
\end{tabular}

Table 1.5: Shown are the quark content for the decaying $B$ mesons and the decay products.

into $K^{+} \pi^{-} 2 / 3$ of the time ${ }^{5}$ while $\phi$ decays into $K^{+} K^{-}$at a rate of $(49.1 \pm 0.8) \%$.

At SLD the reconstruction of charged tracks is of a high quality while the neutral tracks are hard to measure to the desired precision. Thus, the search for rare modes involving a vector meson is limited to cases when the vector decays into two charged tracks, and the sensitivity for detecting a mode involving a $K^{* 0}$ and/or a $\phi$ will be reduced according to the fraction of neutral final states compared to that of charged final states to which this analysis is sensitive. Table 1.5 lists the quark structure of the various particles relevant in this search. All non $B_{s}$ decays that involve two strange quarks in the final state, such as $B^{0} \rightarrow \phi \phi$, are not allowed by the tree level diagrams, and the penguin diagram is expected to be the dominant process. For decays that involve a $K^{* 0}$ the tree level color favored diagrams are excluded, and for $B_{s}$ modes, the decays into a non strange final state, such as $B_{s} \rightarrow \rho \rho$, are not

\footnotetext{
${ }^{5} K^{* 0}$ decays $100 \%$ into a kaon pion pair and an isospin counting gives a rate of $1 / 3$ for neutral modes and a rate of $2 / 3$ for charged modes.
} 


\section{Introduction}

allowed by the tree level diagrams or the penguin diagrams and are governed by the non-spectator diagrams d) and $f$ ). Hence, the various modes have the potential of providing a well balanced understanding of the relevant sub processes.

All the modes will be reconstructed by the charged tracks, and the analysis is based on a full reconstruction of the modes. Other similar modes have not been attempted. Non-resonance modes, for example, such as direct decays of $B^{+}$to $\pi^{+} \pi^{+} \pi^{-}$ and $B^{0}$ to $\pi^{+} \pi^{-} \pi^{+} \pi^{-}$have not been attempted. A degrading of the kinematic signature is expected compared to the resonance modes, and hence will lower the chances of a reconstruction with the high efficiency necessary at SLD (due to the relatively low statistical power). Other resonance modes one can envision are not included in this analysis due to a low decay rate of the corresponding vector meson into charged only final states. On the other hand, time constraints have limited this analysis to include baryon modes such as $B^{0}$ decay into a proton anti-proton pair.

Table 1.6 shows the theoretical predictions for the branching ratios (if any) and the current experimental results as listed in the PDG [1] (if any). The modes $B^{0} \rightarrow$ $P P$ and $B \rightarrow P V$ are measured most precisely at the CLEO experiment and new and updated results are available [24]. In units of $10^{-5}$ they are

$$
B^{0} \rightarrow \pi^{+} \pi^{-}<0.84 \quad B^{0} \rightarrow K^{-} \pi^{+}=1.4 \pm 0.3 \pm 0.1 \quad B^{0} \rightarrow K^{+} K^{-}<0.24
$$




\section{$1.3 \quad$ Rare $B$ Decays}

\begin{tabular}{|c|c|c|c|c|c|}
\hline $\begin{array}{l}\text { Mode } \\
B \rightarrow P P\end{array}$ & $\begin{array}{l}\text { Theory } \\
\text { BR } \times 10^{5}\end{array}$ & $\begin{array}{c}\mathrm{PDG} \\
\mathrm{BR} \times 10^{5}\end{array}$ & $\begin{array}{l}\text { Mode } \\
B^{0} \rightarrow V V\end{array}$ & $\begin{array}{l}\text { Theory } \\
\mathrm{BR} \times 10^{5}\end{array}$ & $\begin{array}{c}\mathrm{PDG} \\
\mathrm{BR} \times 10^{5}\end{array}$ \\
\hline$B^{0} \rightarrow \pi^{+} \pi^{-}$ & $0.5-1.3$ & $<1.5$ & $B^{0} \rightarrow \rho^{0} \rho^{0}$ & $0.06-0.25$ & $<28$ \\
\hline$B^{0} \rightarrow K^{-} \pi^{+}$ & $0.7-2.4$ & $=1.5$ & $B^{0} \rightarrow \bar{K}^{\star 0} \rho^{0}$ & $0.00-0.50$ & $<46$ \\
\hline$B^{0} \rightarrow K^{+} K^{-}$ & - & $<0.5$ & $B^{0} \rightarrow \bar{K}^{\star 0} K^{\star 0}$ & $0.03-0.10$ & - \\
\hline$B_{s} \rightarrow \pi^{+} \pi^{-}$ & - & $<17$ & $B^{0} \rightarrow \phi \rho_{-}^{0}$ & $0.00-0.02$ & - \\
\hline$B_{s} \rightarrow K^{+} \pi^{-}$ & $0.7-0.9$ & $<21$ & $B^{0} \rightarrow \phi \bar{K}^{\star 0}$ & $0.02-1.50$ & $<4.3$ \\
\hline$B_{s} \rightarrow K^{+} K^{-}$ & $0.6-2.1$ & $<5.9$ & $B^{0} \rightarrow \phi \phi$ & - & $<3.9$ \\
\hline \multicolumn{3}{|l|}{$B \rightarrow P V$} & \multicolumn{3}{|l|}{$B_{s} \rightarrow V V$} \\
\hline$B^{+} \rightarrow \rho^{0} \pi^{+}$ & $0.09-0.63$ & $<4.3$ & $B_{s} \rightarrow \rho^{0} \rho^{0}$ & - & - \\
\hline$B^{+} \rightarrow \rho^{0} K^{+}$ & $0.05-0.06$ & $<1.9$ & $B_{s} \rightarrow \bar{K}^{* 0} \rho^{0}$ & $0.00-0.08$ & - \\
\hline$B^{+} \rightarrow K^{* 0} \pi^{+}$ & $0.6-1.0$ & $<4.9$ & $B_{s} \rightarrow \bar{K}^{* 0} K^{* 0}$ & $0.2-0.5$ & - \\
\hline$B^{+} \rightarrow K^{* 0} K^{+}$ & $0.03-0.10$ & - & $B_{s} \rightarrow \phi \rho^{0}$ & $0.00-0.08$ & - \\
\hline$B^{+} \rightarrow \phi \pi^{+}$ & $0.00-0.04$ & - & $B_{s} \rightarrow \phi K^{* 0}$ & $0.00-0.09$ & - \\
\hline$B^{+} \rightarrow \phi K^{+}$ & $0.05-1.60$ & $<1.2$ & $B_{s} \rightarrow \phi \phi$ & $0.02-2.51$ & - \\
\hline
\end{tabular}

Table 1.6: Theoretical predictions and experimental results (PDG) for the branching ratios are shown. The theoretical predictions are found in several publications [23] and the full range of these predictions are listed in this table. Upper limits are at the $90 \%$ confidence level.

$$
\begin{array}{cc}
B^{+} \rightarrow K^{* 0} \pi^{+}<2.7 & B^{+} \rightarrow \rho^{0} \pi^{+}=1.5 \pm 0.5 \pm 0.4 \quad B^{+} \rightarrow K^{* 0} K^{+}<1.2, \\
B^{+} \rightarrow \phi \pi^{+}<0.4 & B^{+} \rightarrow \phi K^{+}<0.6
\end{array}
$$

and line nicely up with theoretical predictions. In connection with the $B^{+} \rightarrow P^{+} V$ modes it is interesting to note the measured result from Delphi [25] for the combined process

$$
\operatorname{Br}\left(B^{+} \rightarrow \rho \pi^{+}, K^{* 0} \pi^{+}\right)=\left(17_{-8}^{+12} \pm 2\right) 10^{-5}
$$

with the central value an order of magnitude larger than predictions. In view of 


\section{Introduction}

this result it will be interesting to see how the SLD measurement turns out. The $B_{s} \rightarrow P P$ modes as listed in the PDG are measured at the Aleph experiment [26]. For the $B_{s} \rightarrow V V$ modes it appear that no measurements have previously been done and for $B_{s}$ modes in general the CLEO experiment and the upcoming $B$-factories will not contribute due to the running at the Upsilon 4s resonance. Hence, the SLD experiment is one out of only a few experiments that has the possibility of measuring rare $B_{s}$ decays. 


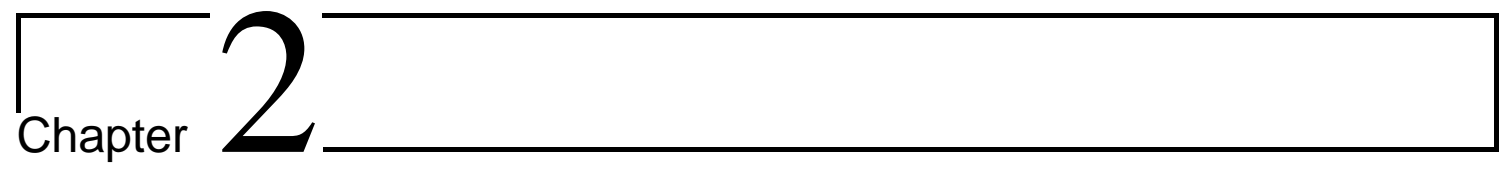

\section{The Accelerator}

The Stanford Linear Collider (SLC) began construction in 1983 and was completed in 1989. This frontier device is a novel kind of machine that serves both as a test bed for a new accelerator technique and as a facility to reach the energy region where the massive $Z$ particle can be produced in quantity and in a simple environment.

The key elements of the SLC (Figure 2.1) are an extensive upgrade to the existing two-mile linear accelerator to produce $50 \mathrm{GeV}$ beams of both electrons and positrons, two small storage rings that are used to damp the beam phase space down to suitable dimensions, two long curving arcs of magnets that are used to transport the separate 


\section{The Accelerator}

electron and positron beams from the end of the linac to a single collision point, and an elaborate focusing system that reduces the sizes of the colliding beams down to dimensions much smaller than a human hair. In 1992 a new polarized source was developed with very high intensity. The following year it was upgraded to generate highly polarized beams, which allow sensitive studies of the $Z$ particle.

The European community has chosen to achieve collisions between $50 \mathrm{GeV}$ electron and positron beams through the use of the more conventional storage-ring technique at the CERN laboratory near Geneva, Switzerland. Their Large Electron Positron collider (LEP) machine is a storage ring some 17 miles in circumference; it has the advantage of four interaction regions (rather than one at the SLC) and the possibility of a higher ultimate energy. The SLC, on the other hand, has a polarized electron beam and a collision spot with an area hundreds of times smaller than that of LEP, which largely compensates for the lower beam crossing rate at SLC.

The pursuit of knowledge in particle physics has, over the past few decades, taken on a truly inhuman scale. Both in terms of the physical size of the equipment and the human effort necessary, a modern particle physics experiment requires the participation and collaboration of many hundreds of physicists, engineers and support staff. For this reason, a comprehensive description of the equipment used in this analysis is beyond the scope of this document. Rather, this chapter will give a broad overview 


\subsection{Stanford Linear Collider}

of the equipment and techniques used in the production of polarized $Z$ bosons at the SLC. References are given throughout the text for further description.

\subsection{Stanford Linear Collider}

The Stanford Linear Collider (SLC) represents a major achievement in particle accelerator technology. The first and only high energy $e^{+} e^{-}$linear collider, the SLC accelerates single bunches of electrons and positrons together down a two-mile linear accelerator and brings them into collision at the center of the SLD detector at a rate

of $120 \mathrm{~Hz}$. Officially proposed in 1980 [34] as a quick and low-cost alternative to the massive storage ring design of LEP, the SLC has endured a somewhat rocky past, including the 1989 Loma Prieta earthquake, but proved early to be a useful research tool in its ability to accelerate polarized electrons. Later, with increased statistics and small beam spots, SLC has been beneficial also for analyses not exploiting the polarization.

The layout of the SLC is shown in Figure 2.1. Two bunches of polarized electrons are produced by photo emission from a strained gallium arsenide cathode and injected into the head of the linac [35]. After a short acceleration section, the 1.2 GeV electron bunches are injected into the North Damping Ring (NDR) where the 


\section{The Accelerator}

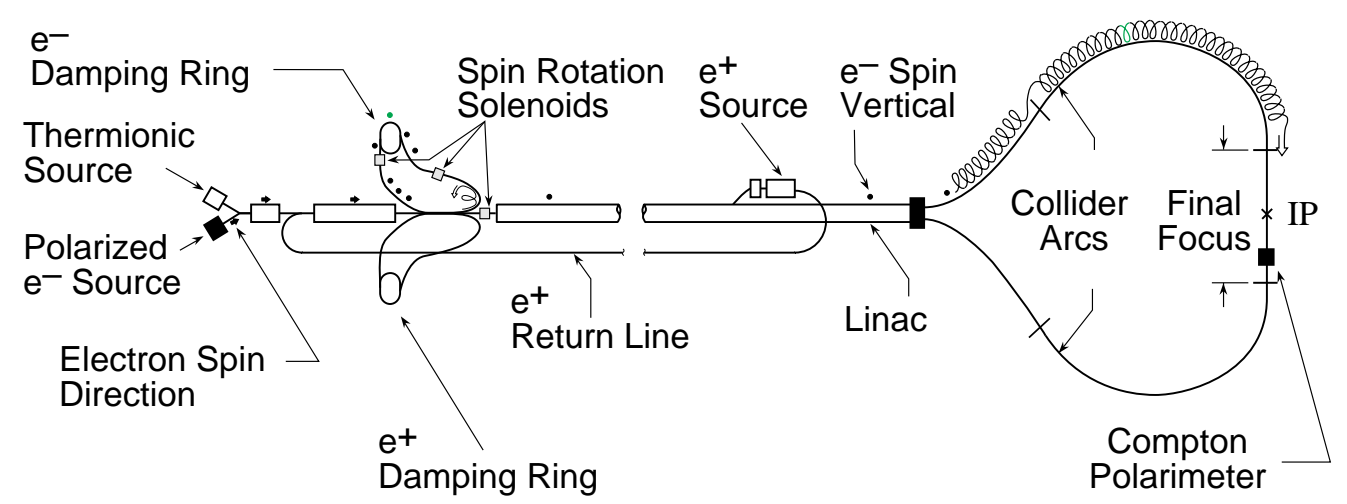

Figure 2.1: The layout of the SLC is shown. Not shown are the beam dumps, which are just past the outgoing final focus section.

phase space (emittance) of the beam is reduced [36]. After a damping time of 8.3 ms, the electrons are extracted from the NDR and injected, along with a bunch of positrons from the South Damping Ring (SDR), into the main accelerating section of the linac. Two thirds of the way down the linac, the trailing electron bunch is stripped off to the positron target, a thick water-cooled chunk of Tungsten-Rhenium alloy [37]. The resulting shower is filtered for positrons which are brought back up to the head of the linac in a separate beam line and injected into the SDR where they are stored for $16.6 \mathrm{~ms}$ waiting for the next machine cycle. After acceleration to around $46.5 \mathrm{GeV}$, the electron and positron bunches are separated in the beam switch yard and fed into the North and South arcs respectively. The $5.6 \mathrm{kGauss}$ dipole magnetic fields in the arcs bend the two beams with a $279 \mathrm{~m}$ effective radius, creating an energy loss of nearly $1 \mathrm{GeV}$ in each beam to synchrotron radiation in 


\subsection{Stanford Linear Collider}

the process, and bringing them to the final focus section and the SLD detector [38].

In the final focus, the two beams are further compressed and focused ${ }^{1}$ for collision at the center of the SLD detector. Most of the beam continues unscathed, traveling upstream through the opposing final focus elements until they are kicked out of the main beam line and dumped.

\subsubsection{Linear Accelerator}

The linear accelerator (linac) used at the SLC is a $3 \mathrm{~km}$ long sequence of 30 conventional copper S-band waveguides each driven by eight 60 MWatt peak power $2856 \mathrm{MHz}$ RF klystrons [39]. An accelerating gradient of $17 \mathrm{MeV} / \mathrm{m}$ is achieved in the copper structures, providing a possible single beam energy of up to $50 \mathrm{GeV}$ in the $3 \mathrm{~km}$ long accelerator.

\subsubsection{Luminosity Overview}

The history of the SLC can in part be viewed as an endless fight to improve the low luminosity ${ }^{2}$ which during the 4 years of running from 1993 to 1996 gave an integrated luminosity of $7.0 p b^{-1}$. During the $1997-98$ run, a luminosity of 12.1

\footnotetext{
${ }^{1}$ Typical cross section for the flat beam at the collision is $2 \mu \mathrm{m}$ by $1 \mu \mathrm{m}$, where the beamsize is largets in the horizontal plane.

${ }^{2}$ Although the first $Z$ was observed as early as 1989 with the Mark II detector, the performance was far from the design goal.
} 


\section{The Accelerator}

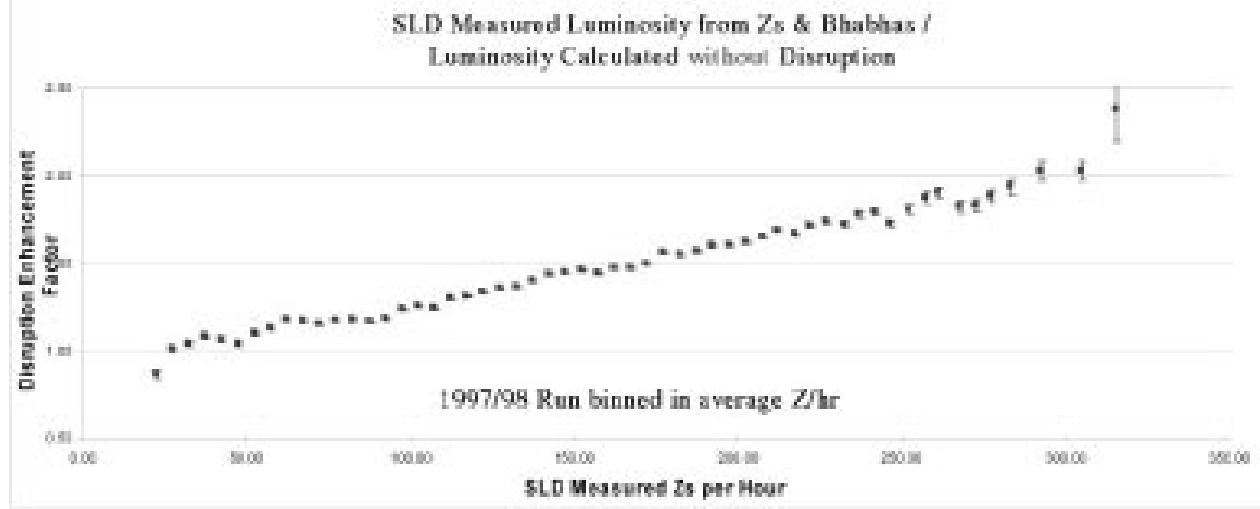

Figure 2.2: The average enhancement factor is shown as a function of luminosity rate.

$p b^{-1}$ was achieved, which gives nearly double the data sample from all previous SLD runs and reflects a substantial increase in performance. The luminosity steadily increased throughout the run bringing the SLC to within a factor of two of design [40]. The improved luminosity in the 1997-98 run was mostly due to changes in tuning procedures and reconfiguration of existing hardware, with only minor upgrade projects. However, it took advantage of earlier hardware upgrades, in particular optics for correcting chromatic aberrations in the final focus, and RF techniques for achieving small emittance from the damping rings.

The luminosity L is given by

$$
L=\frac{N^{+} N^{-} f}{4 \pi \sigma_{x} \sigma_{y}} H_{d},
$$




\subsection{Stanford Linear Collider}

\begin{tabular}{cccccc}
\hline \hline Parameter & & 1993 & $1994-95$ & 1996 & $1997-98$ \\
\hline Horizontal beam size & $\sigma_{x}(\mu \mathrm{m})$ & 2.6 & 2.1 & 2.0 & 1.7 \\
Vertical beam size & $\sigma_{y}(\mu \mathrm{m})$ & 0.8 & 0.6 & 0.8 & 0.9 \\
Horizontal divergence & $\theta_{x}(\mu \mathrm{Rad})$ & 300 & 300 & 370 & 460 \\
Vertical divergence & $\theta_{y}(\mu \mathrm{Rad})$ & 200 & 200 & 260 & 260 \\
Energy spread & $\sigma_{E} / E(\%)$ & 0.30 & 0.12 & 0.16 & 0.13 \\
Bunch intensity & $n_{e} \pm\left(10^{10}\right)$ & 3.0 & 3.5 & 3.5 & 3.5 \\
Electron polarization & $P_{e}(\%)$ & 63.0 & 77.2 & 76.5 & 73.2 \\
Total Luminosity & $L_{t o t}\left(p b^{-1}\right)$ & 1.6 & 3.5 & 1.9 & 12.1 \\
\hline \hline
\end{tabular}

Table 2.1: Typical single beam parameters at the SLC interaction point are listed for the 1993 and 1994-95 running periods. The beam sizes and divergences shown represent single bunch RMS values. The electron beam polarization is luminosity weighted over the course of each running period.

where $N^{ \pm}$are the number of electrons and positrons at the interaction point (current), $f$ is the collision rate, $\sigma_{x, y}$ are the average horizontal $(x)$ and vertical $(y)$ beam sizes, and $H_{d}$ is the disruption enhancement factor which depends on the beam intensities and on the transversal and longitudinal beam sizes. A significant disruption effect was demonstrated for the first time in the 1997-98 run. As beams collide, each beam is focused by the field of the other beam, causing the transverse size to shrink. $H_{d}$ was measured as large as 2 during 1997-98 [41]. See Figure 2.2 for a correlation between the production rate and the enhancement factor.

As the current is limited by instabilities to about $4 \times 10^{10}$ particles per bunch the efforts for increasing the luminosity has been focused on preserving the small emittance $\epsilon$ from the damping rings and on increasing the angular divergence $\theta$ at 
The Accelerator

1992 - 1998 SLD Polarized Beam Running

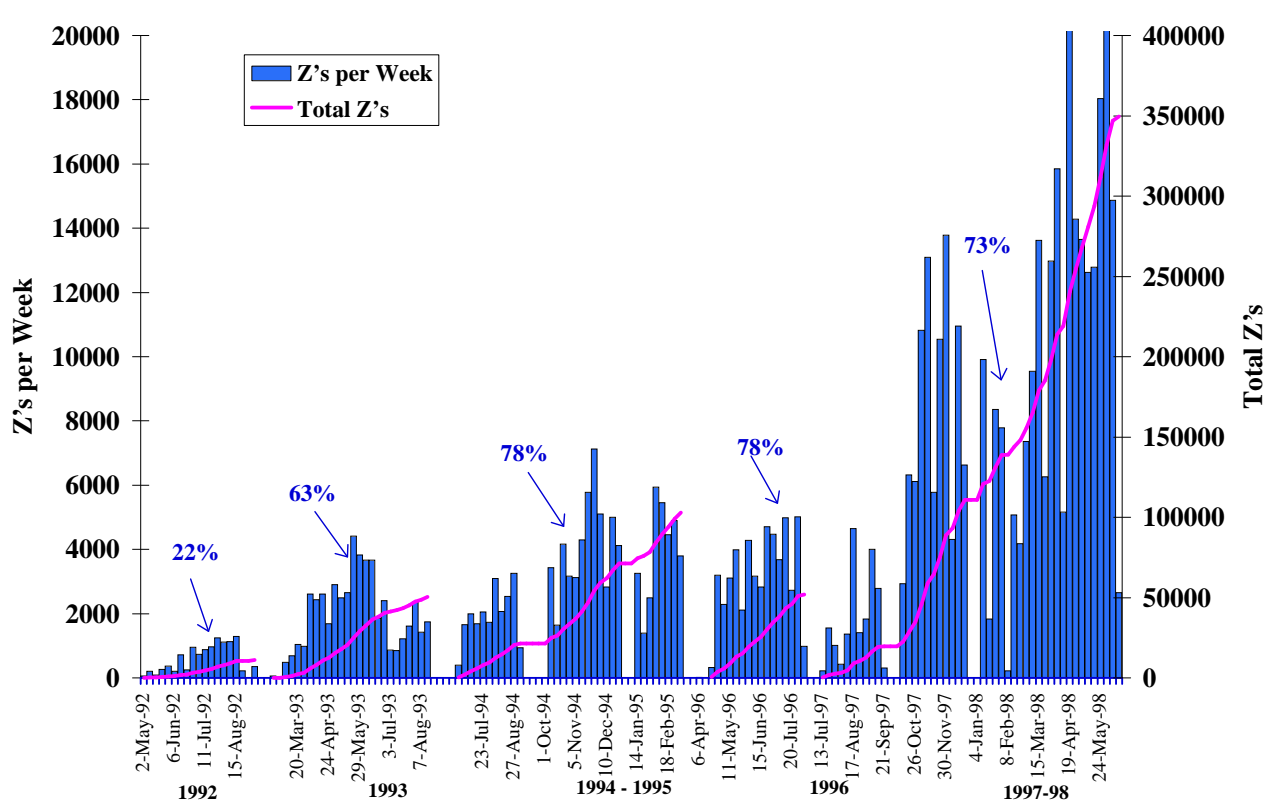

Figure 2.3: A histogram of the $Z$ events over all SLD runs. The histograms shows the accumulated events per week while the solid curve shows the integrated luminosity. The average polarization values are indicated with the arrows.

the SLD IP $\left(\epsilon_{x, y}=\sigma_{x, y} \theta_{x, y}\right)$. The former was mainly achieved by better monitor and feedback systems for the beam transport from the damping rings to the SLD IP which significantly improved chromatic $^{3}$ (aberration) and wakefield ${ }^{4}$ induced emittance

\footnotetext{
${ }^{3}$ The energy-dependent distortion caused by a quadrupole. Particles of different energy are focused to different points. This is because high-energy particles are focused (or bent) less than lower-energy particles. This is analogous to light optics, where light of different colors has different wavelengths (energy) and therefore different indexes of refraction when passing through a thin lens.

${ }^{4}$ The field created by a bunch of charged particles as it passes through the beam pipe. The charges induced in the wall of the pipe by the leading edge of the bunch have an effect (usually bad) on the tail of the bunch. If the beam is centered, wakefields will cancel each other by symmetry, but if the beam is closer to one wall, the tail will be attracted to this wall resulting in a 'banana' shaped bunch (Transverse Wakefield). A wakefield also can be created in RF cavities by the loading effect: the tail gets less energy than the head of the bunch (Longitudinal Wakefield).
} 


\subsection{Stanford Linear Collider}

dilution. At SLC, the maximum achievable angular divergence is limited primarily by backgrounds in the SLD detector. In addition to changing the collimator setup

(masking), the two pairs of super conducting magnet quadruple triplets used for focusing during the 1993-1996 run were accommodated by a set of permanent magnet sextupoles. In February 1998 permanent octupoles were added to the focusing optics. Table 2.1 lists a set of SLC beam parameters for the various runs and Figure 2.3 shows the integrated luminosity per run and the luminosity per week.

\subsubsection{Polarized Electron Source}

It has long been known that polarized electrons can be photo emitted from the surface of a semiconductor, but through the use of a strained-lattice GaAs photo cathode, polarizations in excess of $75 \%$ have been achieved at the SLC [42]. Figure 2.4 shows the energy levels at the top of the valence band and the bottom of the conduction band in strained gallium arsenide (GaAs). An incident circularly polarized photon near the band gap energy of $1.52 \mathrm{eV}$ will excite only the transitions from the top of the valence band into the conduction band as shown. In conventional GaAs, two transitions producing opposite spin electrons are possible from the degenerate $P_{3 / 2}$ energy level at a rate of 3 to 1 as given by the Clebsh-Gordon coefficients. By growing a small layer of GaAs on top of a substrate of gallium arsenide 
The Accelerator

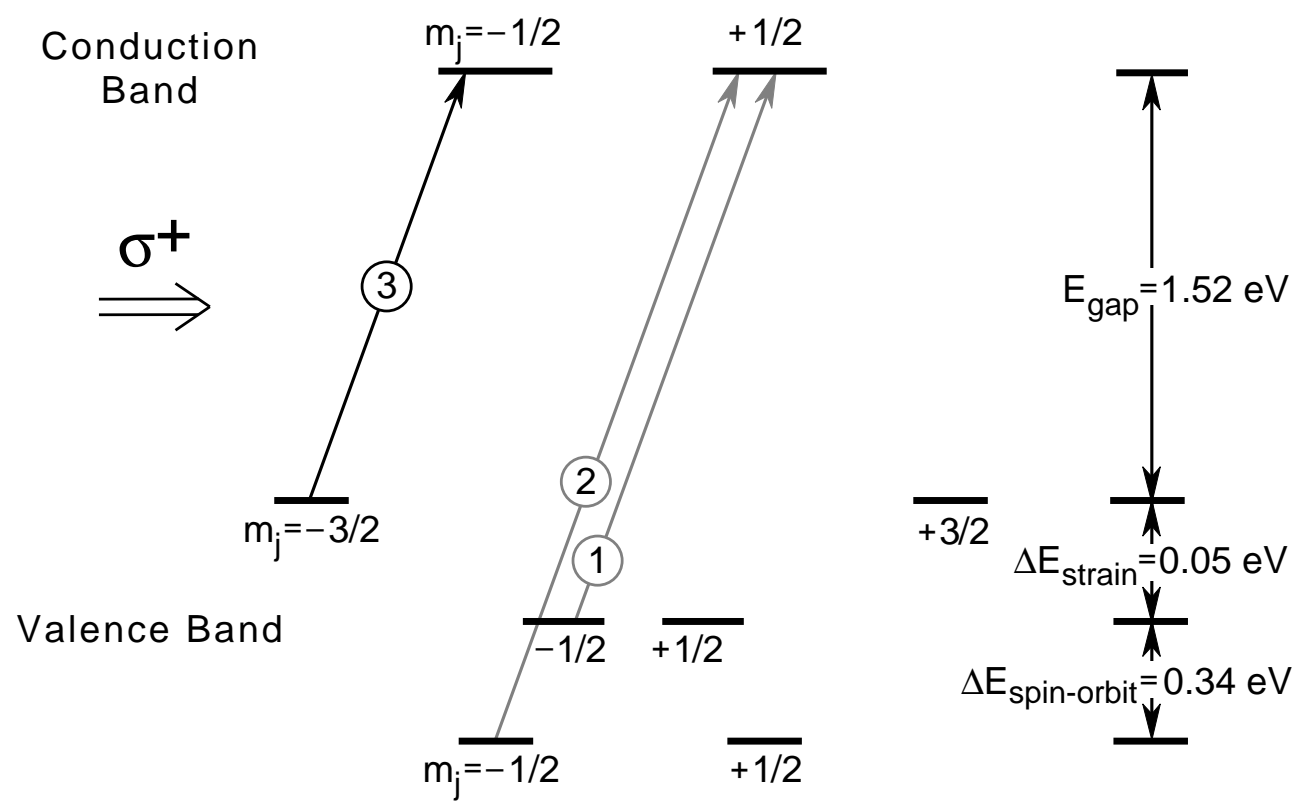

Figure 2.4: The energy levels are shown of the valence band and of the conduction band for strained Gallium Arsenide. The Clebsh-Gordon coefficients for the various spin transitions are also shown for a right handed incident photon. In bulk GaAs, spin-orbit interactions separate the $P_{1 / 2}$ and $P_{3 / 2}$ energy levels to provide a maximum polarization of $50 \%$. With a strained lattice, the degenerate $P_{3 / 2}$ energy levels can also be separated and a polarization of nearly $100 \%$ can be achieved.

phosphide (GaAsP), which has a different lattice spacing than bulk GaAs, the degeneracy in the $P_{3 / 2}$ energy levels can be broken and polarizations above $50 \%$ can be achieved. The exact dimensions of the various cathode layers has a significant effect on the performance of the source due to rescattering of the electrons in the active material, and an improvement in the beam polarization from $63 \%$ in 1993 to over $77 \%$ in 1994 can be directly attributed to reducing the active layer from 300 


\subsection{Stanford Linear Collider}

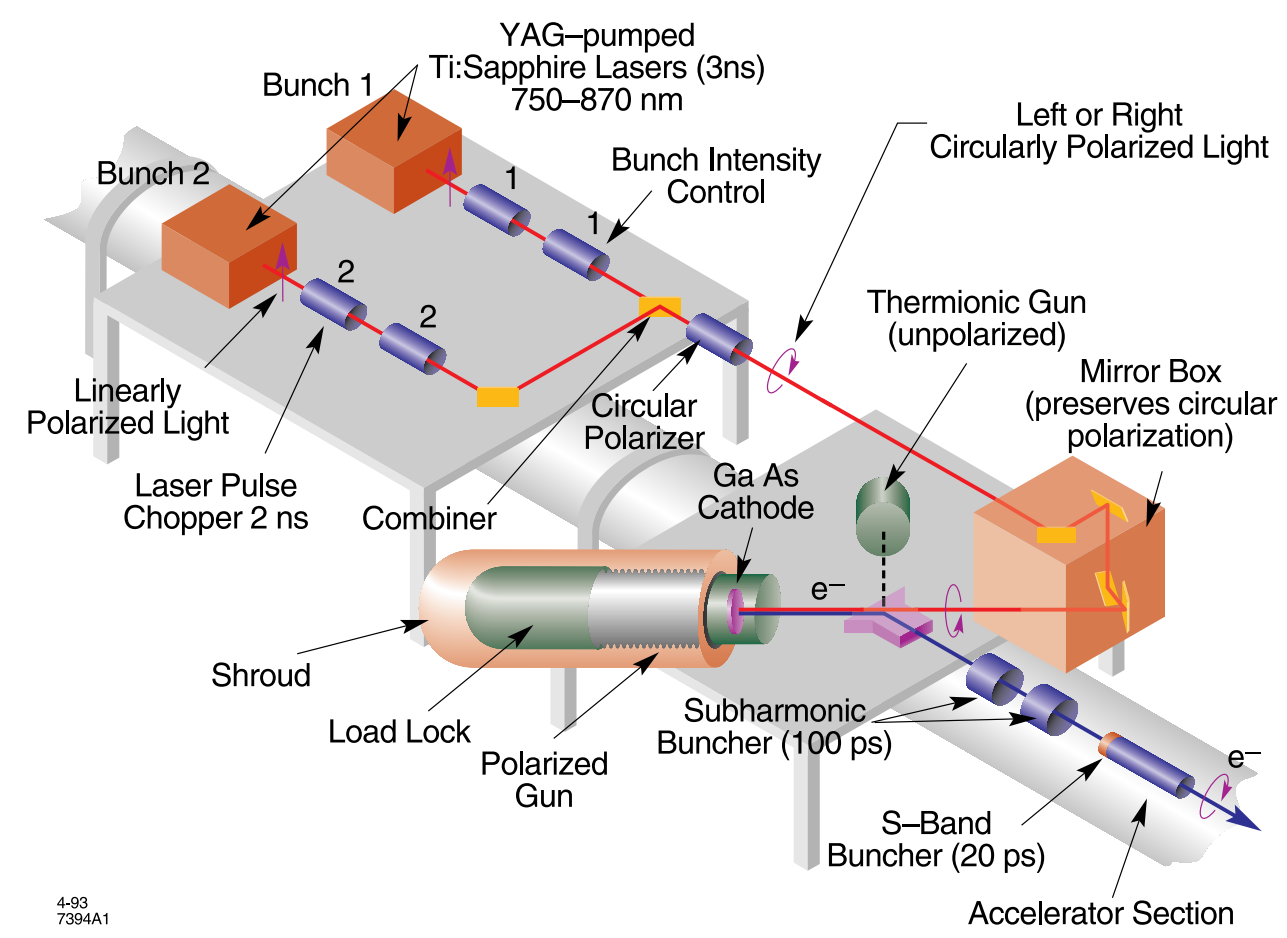

Figure 2.5: Two lasers strike the cathode two times per $120 \mathrm{~Hz}$ machine cycle. One bunch of electrons will eventually come into collision with positrons at the SLD interaction point, while the other electron bunch is used to create positrons. Typically, around $6 \times 10^{10}$ electrons are liberated from the cathode per pulse.

$\mu \mathrm{m}$ to $100 \mu \mathrm{m}$ in thickness [43]. By applying a thin coating of cesium to the face of the photo cathode, the work function of the surface can be reduced, and a modest voltage will extract the polarized electrons from the conduction band. To provide the $6 \times 10^{10}$ electrons needed to drive the SLC, the cathode is maintained near a $\mathrm{QE}$ of $0.4 \%$.

A pair of YAG pumped Ti:Sapphire lasers provide linearly polarized photons which are circularly polarized by use of a Pockels Cell (an active birefringent crystal). 


\section{The Accelerator}

These electro-optic crystals can be used to produce left or right handed light on a pulse by pulse basis by reversing the drive voltage applied. In normal SLC operations the polarization of the incident photons, and hence the polarization of the produced electrons, are selected by a pseudo-random sequence on each machine cycle in an effort to average out any periodicities in the accelerator performance between the two polarization states. The wavelength of the drive laser is empirically set near 860 $\mathrm{nm}$ to maximize the polarization of the produced electrons. Figure 2.5 shows the setup at the electron source.

\subsubsection{Spin Transport}

Electrons are produced longitudinally polarized at the source, and special care must be taken to preserve this polarization as the electrons travel through the accelerator to the SLD. In a magnetic field, the spin precession of a relativistic particle is described by the BMT (Bargmann-Michel-Telegdi) equation, ${ }^{5}$ which for planar motion through a transverse bending field can be written as

$$
\frac{d \theta_{\text {spin }}}{d \theta_{\text {bend }}}=\gamma \frac{g-2}{2},
$$

\footnotetext{
${ }^{5}$ See any graduate-level electromagnetism text.
} 


\subsection{Stanford Linear Collider}

where $\theta_{\text {spin }}$ is the precession angle of the component of the spin vector perpendicular to the magnetic field with respect to the momentum vector, and $\theta_{b e n d}$ is the revolution angle of the particle along its circular path in the bending plane. Note that if $g$ were exactly equal to 2 , the longitudinal polarization of an electron would be maintained as the precession frequency would exactly match the cyclotron frequency. Alternatively, for a mono-energetic beam of electrons at some integer multiple of the 'magic energy' $\gamma=\frac{2 n}{g-2}$, the spin orientation at any particular point along the circular beam orbit will remain unchanged during subsequent revolutions. This is the technique used to measure the muon magnetic moment to high precision.

Because this spin precession is energy dependent, and the SLC electron bunch always has some energy width, any polarization component lying in the bending plane as the electrons enter the North damping ring will be quickly averaged away to zero. To preserve the electron polarization, the combination of a $164^{\circ}$ bend followed by a 6.34 Tesla-meter solenoidal magnetic field region are used to precess the incoming electron polarization vector first transverse to the direction of motion, and then up into the vertical plane. Because this spin rotation was designed for a $1.21 \mathrm{GeV}$ incident electron bunch, but the actual injection is performed at 1.19 $\mathrm{GeV}$, the spin rotation transverse to the bending plane is not perfect. This results in a $1 \%$ polarization loss by the time the electrons are extracted from the NDR. 


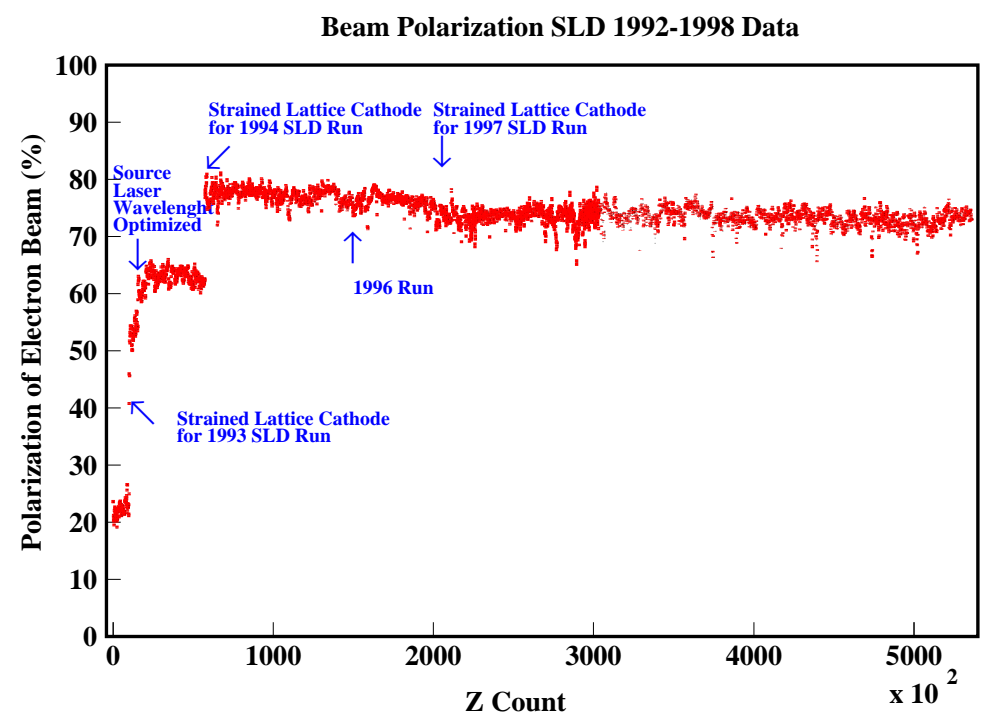

Figure 2.6: The polarization history of the SLC is shown. The measured electron beam polarization is plotted versus the number of hadronic $Z$ decays observed. Each point is the polarization measurement averaged over 1003 minutes Compton runs. The statistical uncertainty is negligible.

An additional pair of spin rotation solenoids were built to allow arbitrary control of the polarization vector at the SLD, however these have not been used since the advent of flat beam running in 1993. A significant increase in luminosity was realized by abandoning a symmetric beam profile and colliding at the SLD with beams which are much broader in the horizontal plane [44]. As a result, however, any solenoidal fields after the damping rings, where the flat beams are created, introduce skew correlations between the horizontal and vertical planes which are generally 


\subsection{Stanford Linear Collider}
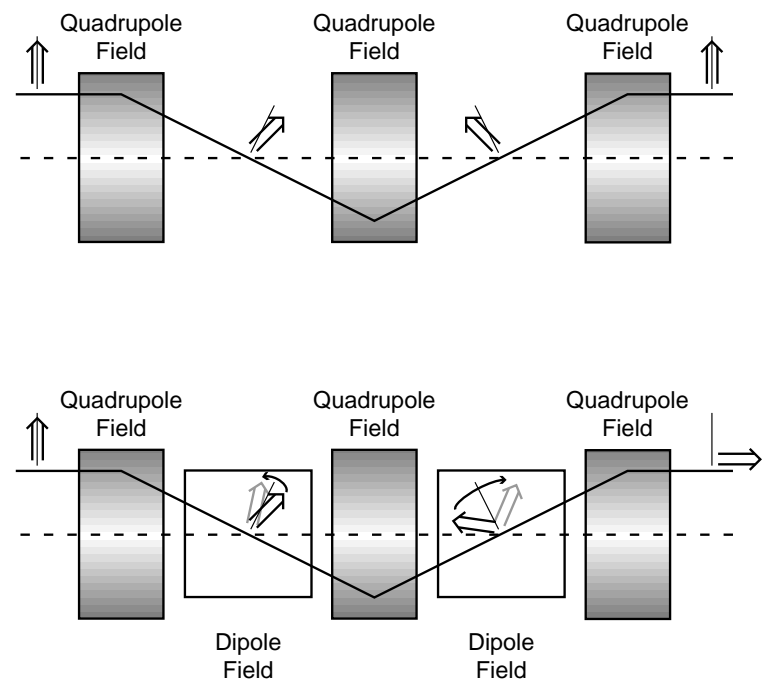

Figure 2.7: The spin orientation of a vertically polarized electron is schematically shown. The first example shows vertical betatron oscillation through a chain of quadrupoles. The spin precession induced by every quad element cancels out preserving the original spin orientation. In the second example, the intermediate vertical dipole fields precess the spin vector so that the next quad precession adds coherently. In this manner, the spin vector can be rotated into the dipole bending plane. The rate of this rotation depends upon the amplitude of the vertical oscillation.

undesirable ${ }^{6}$. Therefore, the electron polarization is left in the vertical plane through

the acceleration process, and the magnetic fields in the North arc are used to align

the final polarization vector at the SLD.

The mechanism for aligning the electron polarization in the arcs is colloquially

known as spin bumps [45]. The SLC North arc is composed of 23 achromats $^{7}$,

\footnotetext{
${ }^{6}$ The solenoid disrupts the beam by coupling the horizontal and vertical emittance, resulting in a rotation of the flat beam in the transverse plan.

${ }^{7}$ The concrete girders, or cells, in the SLC arcs are called 'achromats' because their magnetic optics are designed to be achromatic. A beam line is called achromatic when its beam transport characteristics do not depend on momentum. For convenience of construction and operation, the
} 


\section{The Accelerator}

to provide both bending and focusing of the beam. Quite by accident, the spin precession frequency in each achromat is nearly identical to the betatron oscillation frequency ${ }^{8}$. As shown in Figure 2.7, this near resonance can be exploited to precess the polarization vector out of the vertical plane and into the horizontal plane. Two large betatron oscillations, or spin bumps, are applied to the electron beam late in the arc to rotate the polarization vector into the bending plane where it begins precessing. The amplitude of these two bumps is empirically chosen to maximize the longitudinal polarization at the SLD.

Because the spin precession frequency is energy dependent, the longitudinal polarization of the electron bunch at the SLD is also energy dependent, which causes some problems for the polarization measurement. While the Compton polarimeter measures the polarization of the entire electron bunch, chromatic aberrations in the SLC final focus optics reduce the luminosity generated from the off-energy beam tails. Thus, the energy-dependent spin precession experienced by the electrons in the SLC North Arc gives a systematic lower net longitudinal polarization for the beam tails compared to the beam core. This effect is called the chromaticity effect [46] and systematically lowers the Compton measurement relative to the SLD IP

arcs are divided into 23 subsections, each made up of 20 combined function magnets (dipoles and quadrupoles). Each subsection is called an achromat.

${ }^{8}$ Betatron oscillations are the oscillations about the ideal orbit the electrons experience as they pass through the accelerator magnet lattice. 


\subsection{Stanford Linear Collider}

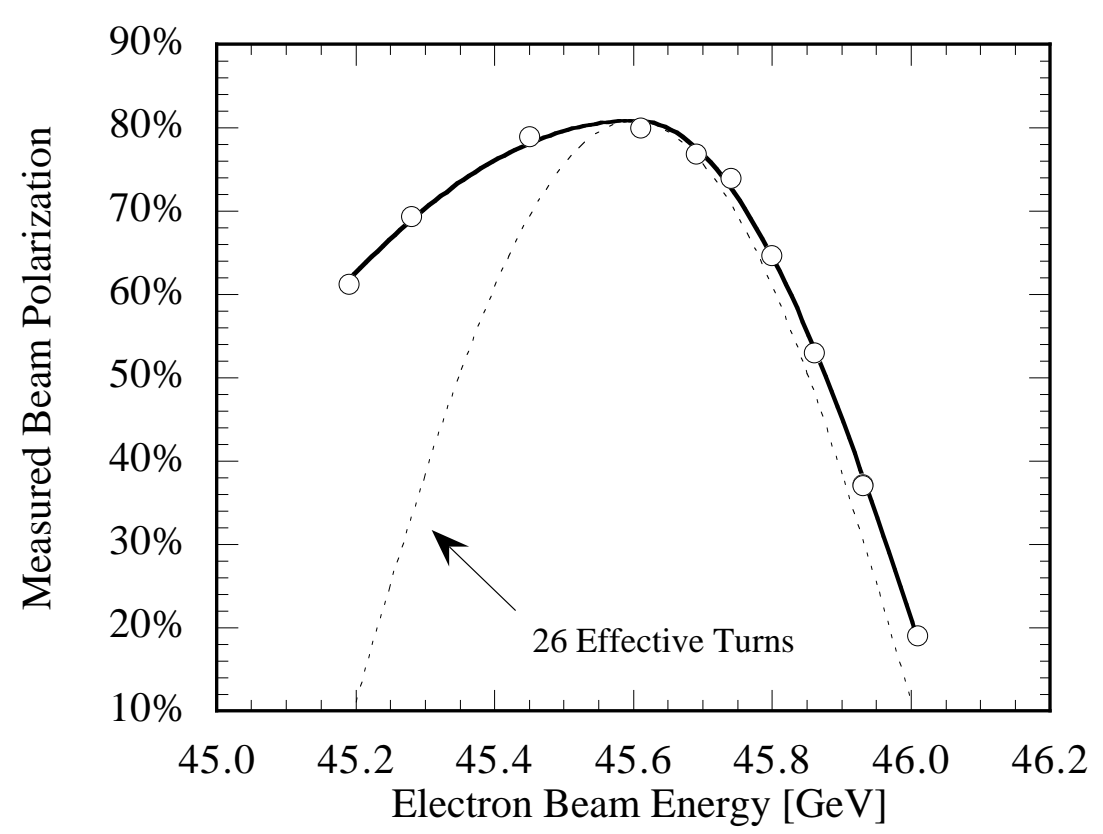

Figure 2.8: The energy dependence of the electron beam polarization was directly measured a number of times during the 1994-95 SLD run. This particular scan was taken in February 1995, near the end of the run. The measured polarization dependence (circles) is weaker than the dependence expected from the spin precession of an electron bunch launched into the arc horizontally polarized (dashed line).

by about $0.1 \%$. The energy dependency grows with the number of spin precessions experienced by the electrons in the North arc, so by keeping the spin orientation vertical for as long as possible the magnitude of this effect can be reduced. At 46 $\mathrm{GeV}$, the spin of an electron launched into the North arc longitudinally polarized will precess a total of 26 times over the net 90 degree bend. Unfortunately, the SLC arcs are not flat, but rather were constructed to follow the bumps and rolls of the surrounding terrain. This convoluted geometry makes an accurate prediction of the 


\section{The Accelerator}

spin dynamics through the North arc spin bumps nearly impossible to simulate, and direct measurements must be made. A second set of spin bumps, set earlier in the North arc, are used to reduce the observed polarization dependence on beam energy. A polarization versus energy scan, shown in Figure 2.8, shows an effective turn number of 12 on the low energy side, although the high energy side shows a much steeper dependence with an effective turn number of 26. As shown, the longitudinal electron polarization is maximized at the beam collision energy near $45.6 \mathrm{GeV}$.

\subsubsection{Energy Spectrometer}

The beam energy at the SLC is measured on each machine pulse with a pair of wire imaging synchrotron radiation detectors (WISRD) [47]. These devices are located in the extraction lines immediately before the beam dumps. Each WISRD consists of three dipole magnets and a pair of copper wire screens. The vertical separation between the synchrotron light swaths emitted by the beam in the first and third horizontally bending dipoles is measured by the wire arrays. Combined with the precisely measured field strength of the intermediate vertically bending analyzing dipole, the beam energy can be deduced. On each machine cycle, the instantaneous energy of each beam can be determined to an error of $22 \mathrm{MeV}$, which is dominated by electronics noise. Averaging over many beam pulses, this error 


\subsection{Stanford Linear Collider}

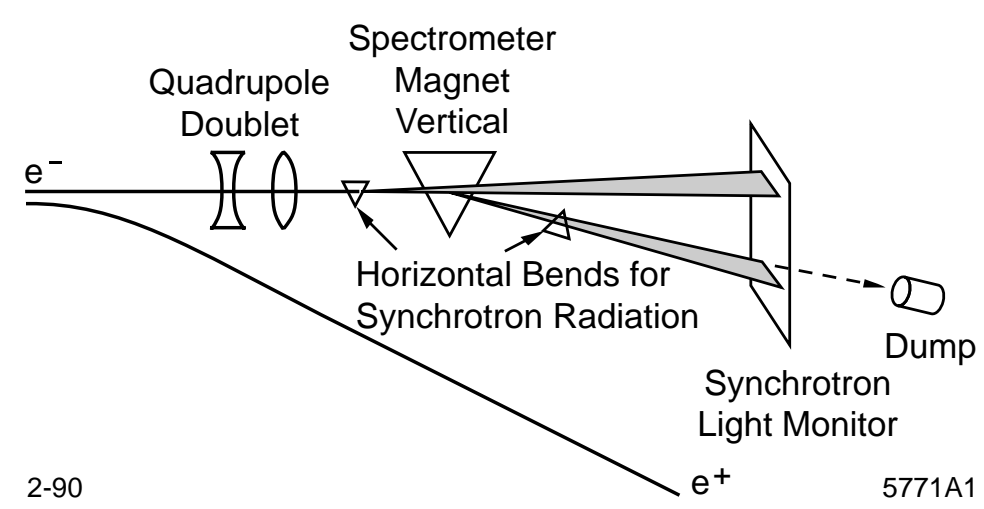

Figure 2.9: The layout of the WISRD energy spectrometer is shown.

can be reduced to about $12 \mathrm{MeV}$ per beam where the remaining irreducible error is dominated by the uncertainty in the wire plane geometry and orientation. Taking this error to be correlated between the two detectors results in a $25 \mathrm{MeV}$ total error on the center of mass collision energy at the SLD. The energy measured for each beam at the WISRD must be corrected by +45 MeV (1993-96) and +40 MeV (199798) to account for synchrotron radiation losses between the SLD and the dump, and by $+5 \mathrm{MeV}(1993-95)+15 \mathrm{MeV}(1997-98)$ to account for additional photons radiated in beam-beam interactions related to the collision process ${ }^{9}$. The luminosity weighted center of mass collision energy for the 1993 and 1994-98 running periods was measured to be $(91.26 \pm 0.02) \mathrm{GeV}$ and $(91.28 \pm 0.02) \mathrm{GeV}$ respectively.

\footnotetext{
${ }^{9}$ These corrections are different for the 1997-98 run compared to the previous runs and reflect the changes to the beam transport to enhance the luminosity.
} 


\section{The Accelerator}

\subsection{Compton Polarimeter}

Located 33 meters downstream from the SLD, the Compton polarimeter is the primary instrument used in measuring the electron beam polarization at the SLC (Figure 2.10). A circularly polarized $2.33 \mathrm{eV}$ photon beam is Compton scattered off the exiting $45.6 \mathrm{GeV}$ electron bunch just before the beam enters the first set of dipole magnets of the SLC South arc heading towards the electron beam dump. These magnets act as a spectrometer sweeping the Compton scattered ${ }^{10}$ electrons out of the main SLC beam line and into a transverse segmented multichannel Čerenkov detector where the momentum spectrum of the electrons is measured in the interval from 17 to $30 \mathrm{GeV}$. Data from the Compton polarimeter are acquired continuously during normal operations of the SLC, providing a $\sim 1-3 \%$ statistical measurement of the electron beam polarization approximately every three minutes.

\subsubsection{Compton Laser}

The scattering 'target' used in the Compton polarimeter is a $\sim 30 \mathrm{~mJ}$ pulse of duration $8 \mathrm{~ns}$ beam of circularly polarized photons produced at $532 \mathrm{~nm}$ by a

\footnotetext{
${ }^{10}$ In this highly boosted interaction the scattered electrons essentially continues straight forward ( $<10 \mu \mathrm{Rad}$ ) but with a reduced energy between $45.6 \mathrm{GeV}$ and $17.0 \mathrm{GeV}$. It is shown [48] that radiative corrections to the Compton cross-section do not alter this energy range.
} 


\subsection{Compton Polarimeter}

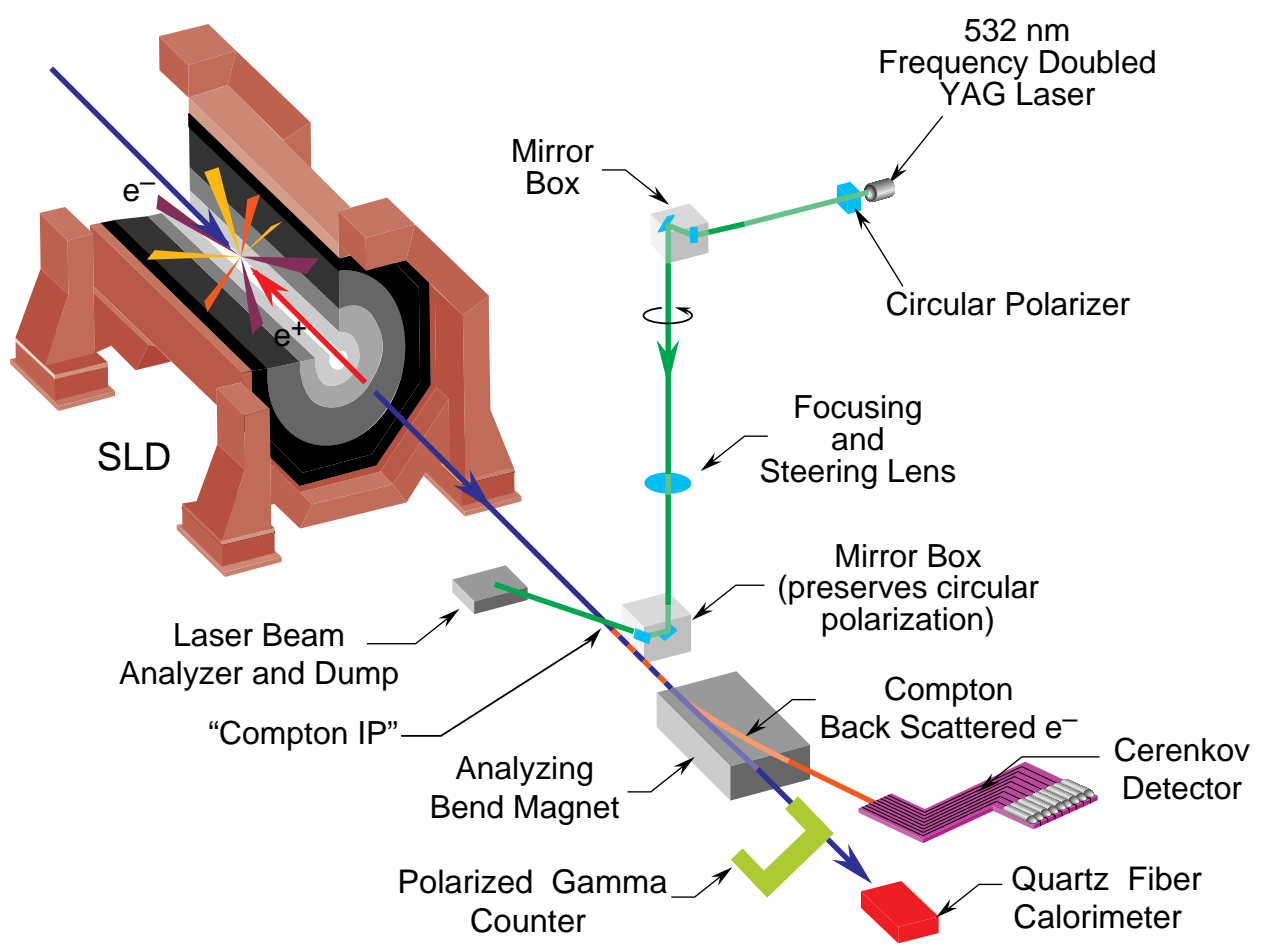

Figure 2.10: The components of the Compton Polarimeter are shown.

Q-switched, frequency doubled Nd:YAG laser running at approximately $17 \mathrm{~Hz}^{11}$.

The circular polarization state of the photon target is selected with a pair of Pockels Cells similar to those used at the electron source, and the polarization of the laser at production is measured with a series of diagnostic photo diodes installed on the laser bench. The laser beam is brought down into the SLC South final focus tunnel through a vent shaft by means of a transport line consisting of four pairs of

\footnotetext{
${ }^{11}$ To avoid possible synchronization effects with the SLC machine the Compton laser is fired at every 7 th beam pulse with occasional increase in the laser rate (every sixth beam pulse) such as to match two beam pulses with 5 beam pulses in between.
} 


\section{The Accelerator}

phase-compensated mirrors. The photons enter the SLC beam line through a low birefringence window, and collide with the outgoing electrons at a crossing angle of $10 \mathrm{mRad}$. The timing of the $8 \mathrm{~ns}$ long laser pulse must be carefully maintained to optimally coincide with the $3 \mathrm{ps}$ long electron bunch, and a series of movable lenses and mirrors are used to keep the $\sim 1 \mathrm{~mm}$ diameter photon beam centered on the outgoing $\sim(1.0 \times 0.4) \mathrm{mm}^{2}$ electron bunch. The laser beam continues out of the SLC vacuum enclosure and into an analysis box in the SLC tunnel where the polarization of the photons can be analyzed by a series of optical polarization filters.

\subsubsection{Electron Transport}

Due to the large boost from the incident $45.6 \mathrm{GeV}$ electron bunch, the scattered electrons remain with the main outgoing beam until the first bending element of the SLC South arc is reached. The Compton scattering cone in the lab frame of $\sim$ $10 \mu \mathrm{Rad}$ is, in fact, insignificant when compared to the $\sim 80 \mu \mathrm{Rad}$ horizontal and $\sim$ $25 \mu \mathrm{Rad}$ vertical beam divergence at the Compton interaction point. Two beamline dipoles and one beamline quadrupole magnets introduce a momentum dependent kink in the outgoing electron trajectories. The off-energy Compton electrons are thus swept out of the main SLC beam line, exiting the SLC vacuum enclosure through a thin stainless steel window, and into the Compton Čerenkov Detector (CKV). The 


\subsection{Compton Polarimeter}

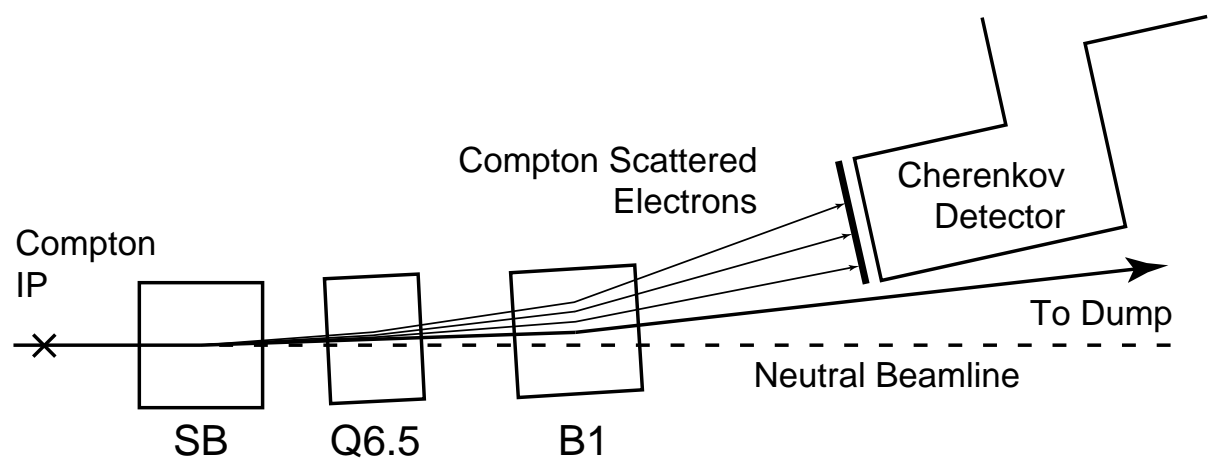

Figure 2.11: The spectrometer used in the Compton polarimeter is shown. The Q6.5 quadrupole was added before the 1994-95 run as part of an SLC final focus upgrade. This figure is not drawn to scale.

path of the Compton scattered electrons through the spectrometer elements and into the CKV detector is shown schematically in Figure 2.11.

\subsubsection{Compton Čerenkov Detector}

Shown in Figure 2.12, the CKV is a segmented threshold Čerenkov counter with nine readout channels instrumented with Hamamatsu R1398 photo multiplier tubes. Originally filled with cis- and trans-2-butene, the radiating gas was changed near the start of the 1994 run to propane which provides better resistance to radiation damage and polymerization which was believed to be degrading the detector. At a slight overpressure of 1.1 atmospheres, this gas provides a Čerenkov threshold for relativistic electrons at roughly $10 \mathrm{MeV}$ which is crucial for avoiding the abundance 


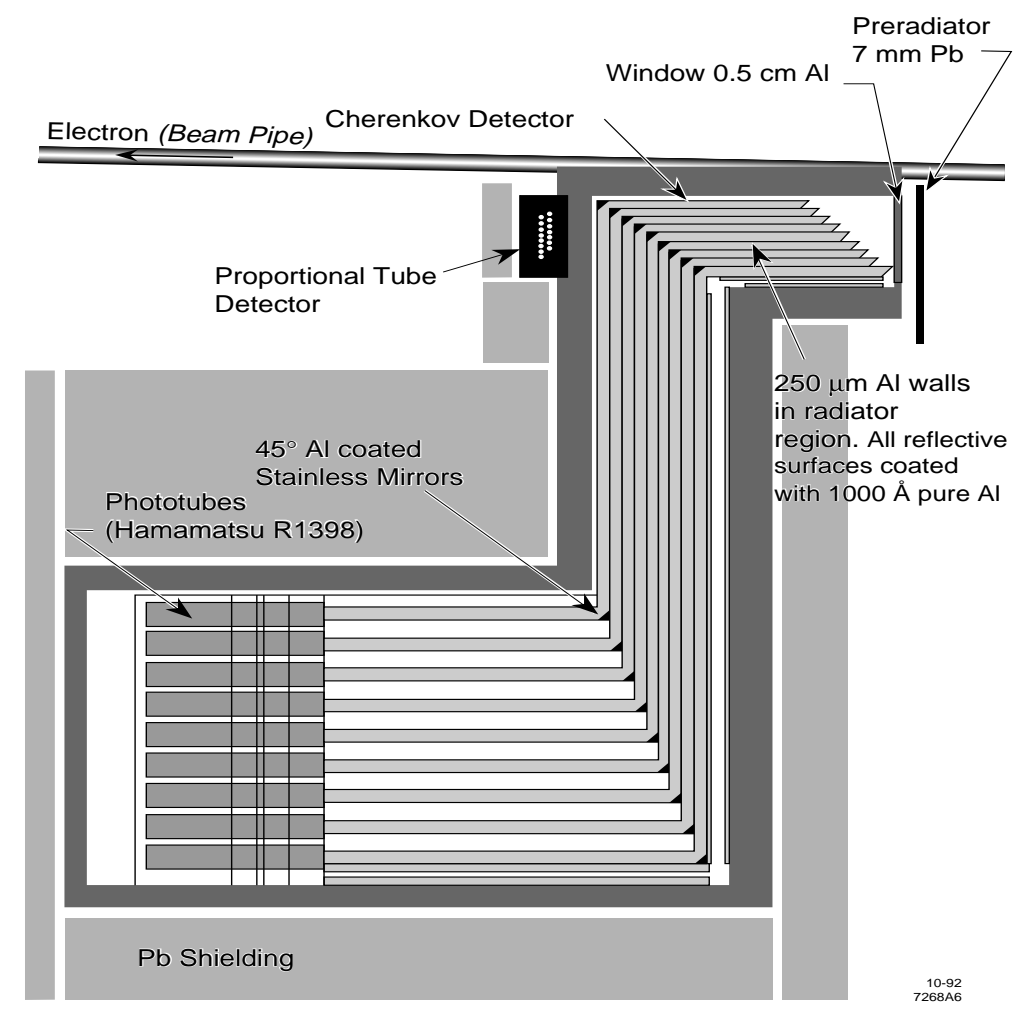

Figure 2.12: The layout of the multi-channel Čerenkov detector for the Compton Polarimeter is shown.

of low energy $(<2 \mathrm{MeV}$ ) background associated with the main electron beam. Relativistic electrons passing through the CKV detector produce UV Čerenkov photons at a characteristic polar angle of $55 \mathrm{mRad}$ which are reflected by thin aluminum vanes away from the beam line and into the heavily shielded photo multiplier tubes. To boost the signal seen in the CKV, a $6.86 \mathrm{~mm}$ preradiator is placed immediately in front of the detector face, providing a signal amplification factor of $\sim 4$.

The two additional detectors in Figure 2.12, the polarized gamma counter [50] 


\subsection{Compton Polarimeter}

(PGC) and the quartz fiber calorimeter [51] (QFC), makes use of the Compton backscattered photons in measuring the electron beam polarization. Due to large sensitivity to beamstrahlung photons from the $e^{+} e^{-}$collisions these detectors only operate during dedicated electron only runs and provide a $0.4 \%$ cross-check on the CKV detector polarization measurement.

\subsubsection{The Polarization Measurement}

The Compton asymmetry in each CKV detector channel $A_{C}^{i}$ is defined to be the asymmetry in the scattering cross section when the electrons and photons collide with their polarization vectors aligned forming a $J_{z}=3 / 2$ spin state, and collisions with their polarization vectors opposed forming a $J_{z}=1 / 2$ spin state. This asymmetry can be written as

$$
\begin{aligned}
A_{C}^{i} & \equiv \frac{\sigma^{i}\left(J_{z}=3 / 2\right)-\sigma^{i}\left(J_{z}=1 / 2\right)}{\sigma^{i}\left(J_{z}=3 / 2\right)+\sigma^{i}\left(J_{z}=1 / 2\right)} \\
& =\left|P_{e}\right|\left|P_{\gamma}\right| a^{i},
\end{aligned}
$$

where it is understood that $P_{e}$ denotes the longitudinal electron polarization, and $a^{i}$ is the analyzing power of each CKV channel which is given by

$$
a^{i}=\frac{\left.\int \frac{d \sigma}{d x}\right|_{\text {unpol }} A_{e \gamma}^{z}(x) R^{i}(x) d x}{\left.\int \frac{d \sigma}{d x}\right|_{\text {unpol }} R^{i}(x) d x} .
$$




\section{The Accelerator}

This analyzing power $a^{i}$ is the cross section weighted asymmetry integrated across the response function $R^{i}(x)$ for the particular channel, normalized to the total channel response.

From Equation 2.3, the measurement of the electron polarization requires four components:

- The raw asymmetry $A_{C}^{i}$ measured in a particular CKV detector channel;

- the theoretical Compton asymmetry $A_{e \gamma}^{z}(x)$ as well as the unpolarized Compton cross-section $\sigma_{\text {unpol, }}$ precisely calculable within QED;

- the channel response function $R^{i}(x)$, determined by a calibration procedure;

- the circular polarization $P_{\gamma}$ of the incident laser beam.

Figure 2.13 shows the Compton asymmetry expected if the electron beam were $100 \%$ polarized (dashed line) and similarly for $77 \%$ electron polarization (solid line). The circular points show the approximate positions of the seven active Cerenkov channels, the ones within reach of the Compton kinematics between 17 and 45 $\mathrm{GeV}$. The polarization of the electron beam is then found independently by the various channels as essentially the ratio of the measured asymmetry to the expected measurement if the beam were $100 \%$ polarized. 


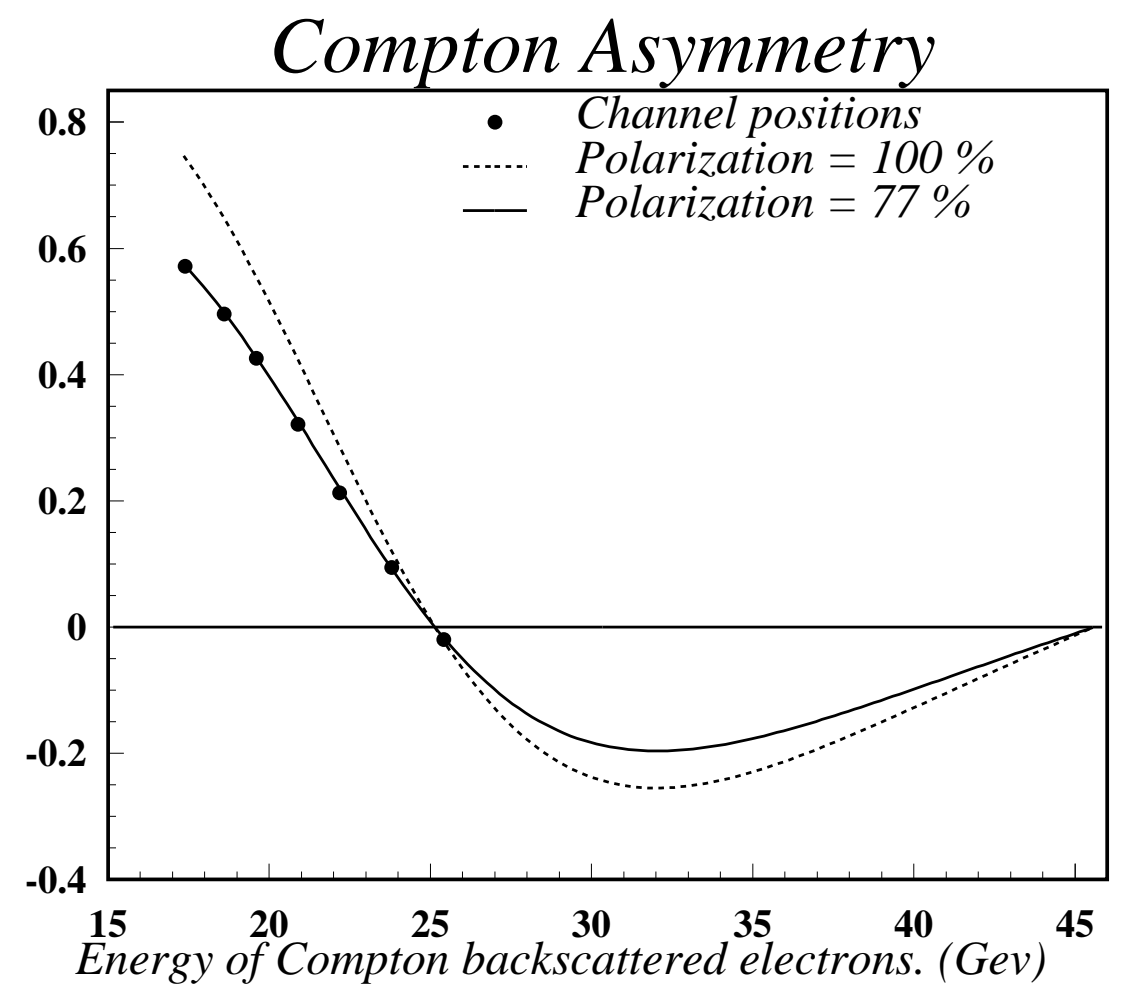

Figure 2.13: The Compton asymmetry versus the energy of the back-scattered Compton electrons is shown for $100 \%$ and $77 \%$ polarization.

The polarization measurements run parasitically with SLD collisions and one measurement takes three minutes. In order to measure the polarization for intervals that involves production of Z's, a luminosity weighted polarization average is formed. Each hadronic event ${ }^{12}$ is associated with a unique polarization value. This is the polarization measurement closest in time to the relevant hadronic event. It requires that a polarization measurement can be found within \pm 1 hour time limit and it

\footnotetext{
${ }^{12}$ Defined here as the events used in the left-right polarization asymmetry $\left(A_{l r}\right)$ analysis $[6]$.
} 


\section{The Accelerator}

is also required that the individual polarization error is less than $3 \%$ for it to be considered in this matching. If no matching is possible the $Z$ event is dropped. The arithmetic ${ }^{13}$ average of all such polarization measurements (one for each hadronic event) then forms the luminosity weighted average. With the improved luminosity at the SLC for 1997-98, which was partly due to improved beam focusing at the SLP IP, the Compton polarimeter has seen larger backgrounds compared to previous runs. Many improvements were done to enhance the signal to noise ratio, including increased Compton laser intensities, improved beam transport from the SLD IP to the Compton IP to minimize beam losses and the execution of dedicated runs without the positron beam, roughly every one hour under noisy conditions. Even with the mentioned improvements the background levels are fairly extensive during parts of the 1997-98 run and large statistical fluctuations in the measured polarization can be seen. In order to accommodate such variations the luminosity weighted polarization average is now calculated as the arithmetic average of the following polarization values

$$
\begin{aligned}
P_{\text {hadronic event }} & =\frac{1}{\Lambda} \sum_{i} \frac{e^{-\frac{|\Delta t|^{2}}{2(\delta t)^{2}}} \times P_{i}}{\left(\delta P_{i}\right)^{2}}, \\
\Lambda & =\sum_{i} \frac{e^{-\frac{|\Delta t|^{2}}{2(\delta t)^{2}}}}{\left(\delta P_{i}\right)^{2}},
\end{aligned}
$$

\footnotetext{
${ }^{13}$ Arithmetic average is simply the unweighted average $\langle x\rangle=1 / N \sum_{i=1}^{N} x_{i}$.
} 


\subsection{Compton Polarimeter}

\begin{tabular}{clllll}
\hline 1992 & $P_{e}=0.224$ & \pm 0.006 & \\
1993 & $P_{e}=0.626$ & \pm 0.012 & \\
$1994-95$ & $P_{e}=0.772$ & \pm 0.005 & \\
1996 & $P_{e}=0.765$ & \pm 0.005 & \\
1997 & $P_{e}=0.733$ & \pm 0.008 & Preliminary \\
1998 & $P_{e}=0.731$ & \pm 0.008 & Preliminary \\
\hline
\end{tabular}

Table 2.2: Luminosity weighted polarization results. The 1997-98 results are preliminary. where $P_{i}$ is all the individual ( 3 minutes) polarization measurements within $|\Delta t|$ (maximum one hour) of each hadronic $Z$ event and with statistical uncertainty $\delta P_{i}$ (maximum $10 \%)$. The Gaussian function ( $\delta t=20$ minutes) provides for time filtering by giving more weights to measurements that are close in time to the measured hadronic events. Hence, for each hadronic $Z$ event we form a time weighted polariza-

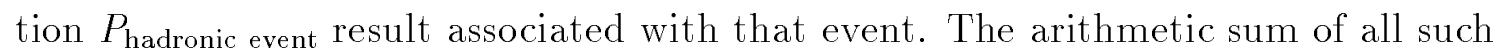
Gaussian weighted polarization values forms the luminosity weighted polarization average. Table 2.2 lists the weighted electron polarization results. Statistical uncertainties are here negligible and the uncertainties listed are strictly from systematic effects. The analysis for the 1997-98 run has yet to be finalized and the uncertainties are kept conservatively large.

Table 2.3 lists the various sources to the uncertainties. The 'Compton IP $\leftrightarrow$ SLD IP' contribution is a combination of the uncertainty in the chromaticity effect (Section 2.1.4), as well as a small correction for spin precession in the outgoing final 


$$
\text { Polarization Uncertainty }\left(\frac{\delta P_{e}}{P_{e}} \times 10^{2}\right)
$$

\begin{tabular}{ccccccc}
\hline Uncertainty & 92 & 93 & $94-95$ & 96 & 1997 & 1998 \\
\hline Laser Polarization & 2.0 & 1.0 & 0.20 & 0.20 & 0.20 & 0.10 \\
Detector Linearity & 1.5 & 1.0 & 0.50 & 0.50 & 0.50 & 0.50 \\
Detector Calibration & 0.4 & 0.5 & 0.29 & 0.30 & 0.30 & 0.30 \\
Electronic Noise & 0.4 & 0.2 & 0.20 & 0.20 & 0.20 & 0.20 \\
Interchannel Consistency & 0.9 & 0.5 & - & - & 0.80 & 0.80 \\
\hline Total Polarimeter Uncertainty & 2.7 & 1.6 & 0.64 & 0.64 & 1.03 & 1.01 \\
\hline Compton IP \& SLD IP & - & 1.1 & 0.17 & 0.18 & 0.07 & 0.08 \\
\hline Total $P_{e}$ Uncertainty & 2.7 & 1.9 & 0.67 & 0.67 & 1.03 & 1.01 \\
\hline
\end{tabular}

Table 2.3: A detailed list of the systematic uncertainties for the polarization measurement. The 1997-98 results are preliminary.

focus quadupoles. It has been shown in a recent study [49] that the detector linearity uncertainty are less than $0.2 \%$ and the final numbers will be adjusted accordingly to this. Further improvements for the interchannel consistency are expected to bring the uncertainty on the $1997-98$ result in line with the 1996 result. For further reading on the Compton polarimeter see [52]. 


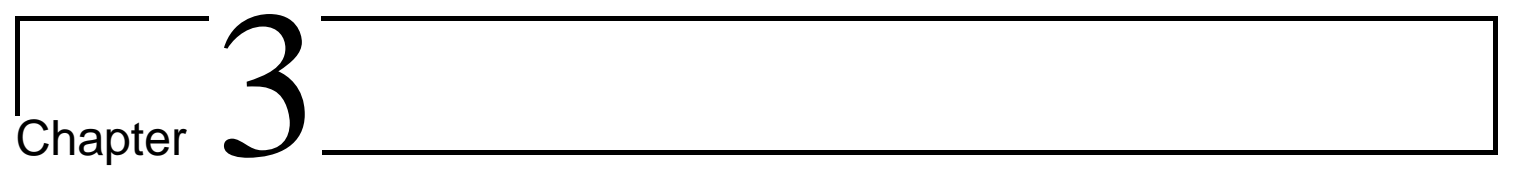

\section{The SLD Detector}

The goal of the SLC Large Detector (SLD) is the physics analysis of $Z$ particles produced with the SLC, in sufficient quantity and quality to make significant contributions to the understanding of particle physics. Originally proposed in 1984 [53] and completed in 1991, SLD is a state-of the art general purpose solenoidal particle detector designed to exploit the capabilities of the SLC in order to study physics at the $Z$ peak. An isometric view of the SLD is shown in Figure 3.1 and a quadrant view is shown in Figure 3.2 .

The various SLD subsystems shown provide simultaneous measurements of the charge, momentum, energy and species of the observable particles created by the 


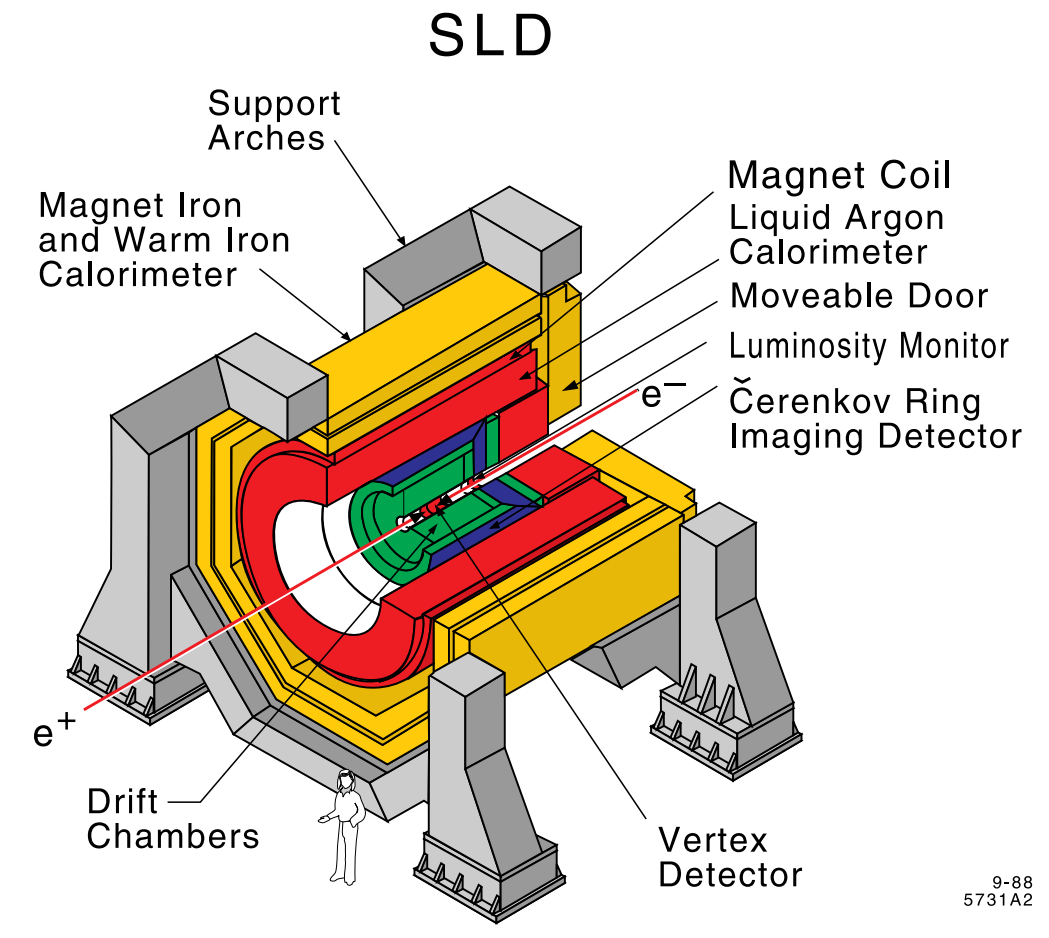

Figure 3.1: The SLD Detector is shown in a cutaway view with the endcaps removed for clarity.

decay of $Z$ bosons. The luminosity measurement is done with the appropriately named Luminosity Monitor which measures the well understood small angle Bhabha scattering rate, which is calculable in QED. Particle identification of pions, kaons and protons are achieved with the Čerenkov Ring Imaging Detector by prior knowledge of the track momenta combined with a measurement of the track velocity via the radius of the Čherenkov cone. On a general note, only muons penetrate the bulk of the detector, and are therefore identified in the Warm Iron Calorimeter. The Vertex 


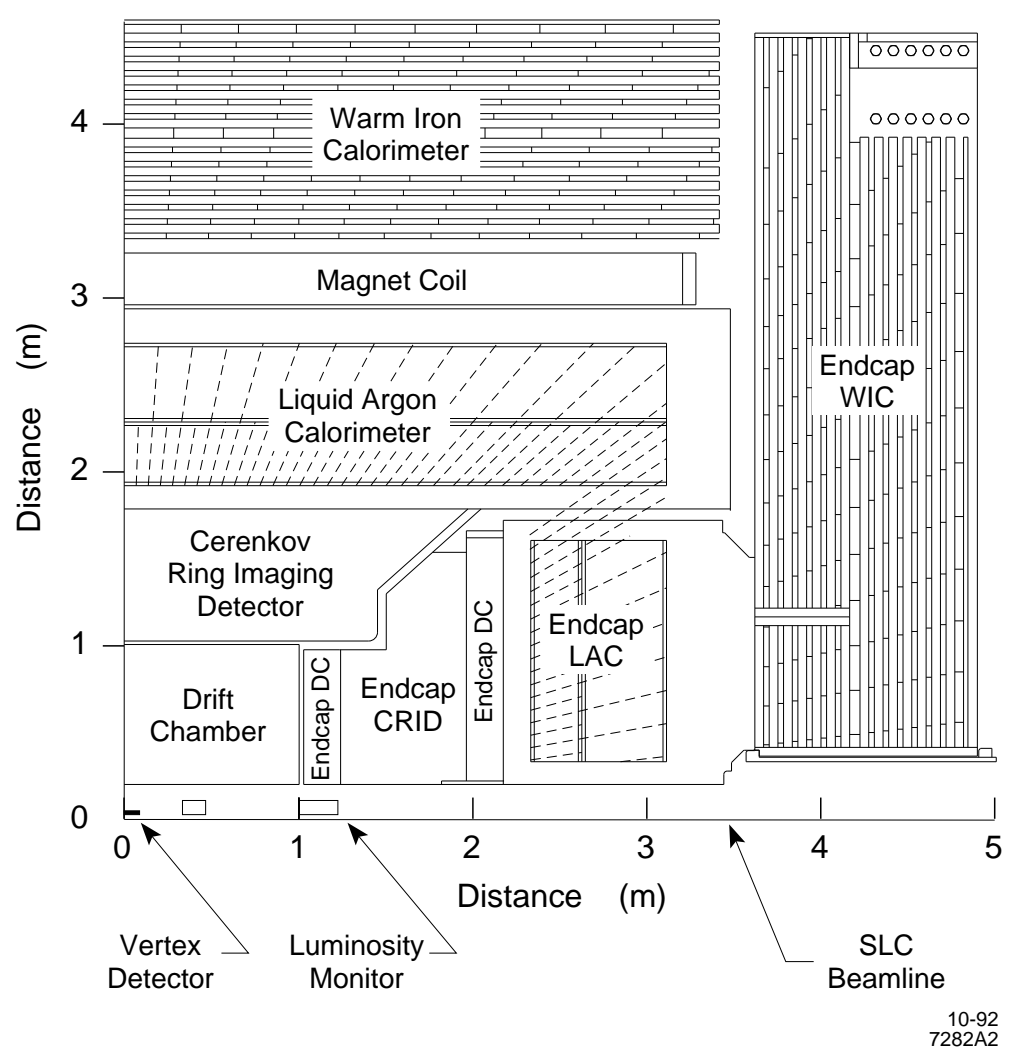

Figure 3.2: The SLD detector is shown in quadrant view with the IP at the lower left corner. The detector is designed to be both radially and longitudinally symmetric.

Detector and the Drift Chamber reconstruct charged track momenta and trajectories in the 0.6 Tesla field from the Magnet Coil. The Liquid Argon Calorimeter provides an energy measurement of all tracks except neutrinos and muons by measuring the absorbed energy when the particle showers in the calorimeter.

This chapter will give an overview of the various sub detectors. To some degree extra emphasis is put on the charged track reconstruction for its importance to the 


\section{The SLD Detector}

analysis described in this thesis.

\subsection{Luminosity Monitor}

As the SLD data size has increased significantly, statistics necessary to make competitive measurements of absolute cross sections has been achieved. The luminosity information has therefore moved from merely being a diagnostic tool to an vital input to physics results.

The SLD luminosity monitor small angle tagger (LMSAT) is a pair of compact, finely segmented silicon-tungsten calorimeters installed immediately around the beam pipe on either side of the SLD IP [54]. The LUM is used to identify low-angle Bhabha scattering between $23 \mathrm{mr}$ and $68 \mathrm{mr}$ in polar angle, which is dominated by the well understood electromagnetic (QED) process of $e^{+} e^{-}$t-channel

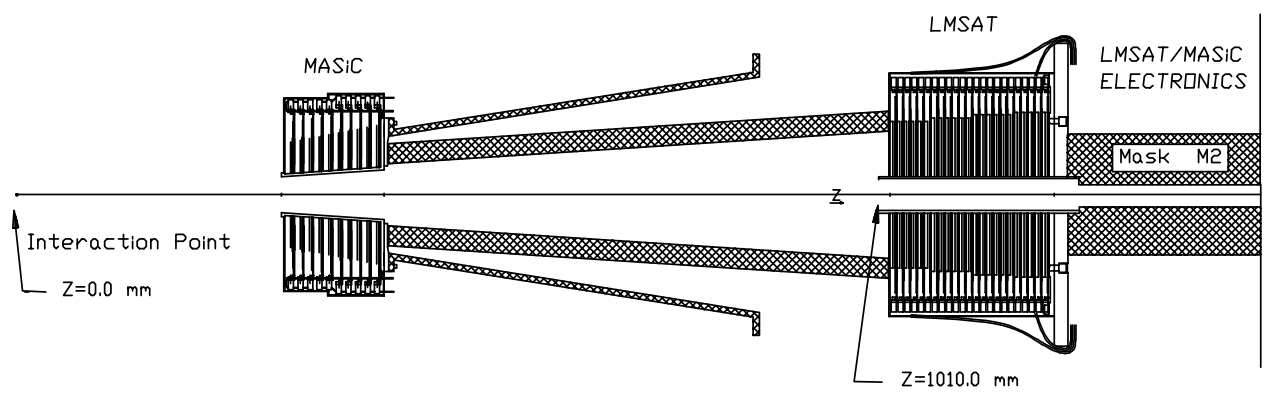

Figure 3.3: A side view of the Luminosity Monitor Small Angle Tagger (LMSAT) and the Medium Angle Silicon Calorimeter (MASC). 


\subsection{Luminosity Monitor}

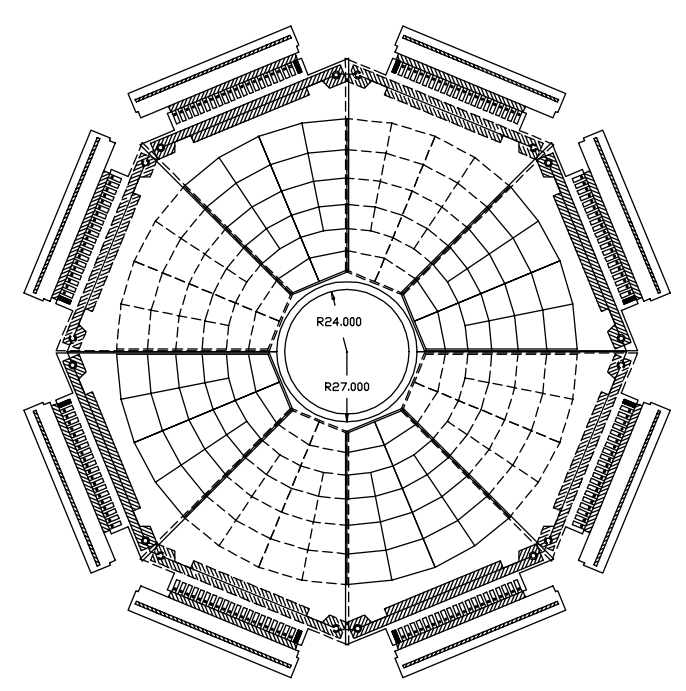

Figure 3.4: One silicon layer of the SLD Luminosity Monitor Small Angle Tagger as seen from the interaction point.

photon exchange. The LUM is triggered and read out at $120 \mathrm{~Hz}$ as part of the LAC data acquisition to provide a dead-time free count of the number of low-angle bhabha events which can then be used to estimate the total luminosity delivered by the SLC.

The LMSAT is shown in Figure 3.3. It consists of 23 longitudinal layers of alternating silicon detectors and tungsten radiators. Electromagnetic showers which develop in the tungsten create electron/hole pairs in the fully depleted silicon detectors which are then collected by charge sensitive preamplifiers. The silicon samples $1.54 \%$ of the shower, based on the $\mathrm{dE} / \mathrm{dx}$ sampling fraction for minimum ionizing particles. The total depth of the calorimeter is 21 radiation lengths, containing > 


\section{The SLD Detector}

\begin{tabular}{cccc} 
& \multicolumn{3}{c}{ Integrated Luminosity $\left(\mathrm{pb}^{-1}\right)$} \\
\hline 1993 & 1.777 & $\pm 0.005($ stat $)$ & $\pm 0.018($ sys $)$ \\
$1994-95$ & 3.699 & $\pm 0.007($ stat $)$ & $\pm 0.037($ sys $)$ \\
\hline VXD2 & 5.476 & $\pm 0.004($ stat $)$ & $\pm 0.055($ sys $)$ \\
\hline 1996 & 1.860 & $\pm 0.005($ stat $)$ & $\pm 0.019($ sys $)$ \\
1997 & 3.790 & $\pm 0.007($ stat $)$ & $\pm 0.037($ sys $)$ \\
1998 & 8.321 & $\pm 0.011($ stat $)$ & $\pm 0.083($ sys $)$ \\
\hline VXD3 & 13.971 & $\pm 0.006($ stat $)$ & $\pm 0.140($ sys $)$ \\
\hline
\end{tabular}

Table 3.1: Listed are the SLC luminosity results for the various run periods. The combined results for the periods with the vertex detectors VXD2 and VXD3 are also shown.

$99.5 \%$ of a $45 \mathrm{GeV}$ electromagnetic shower. Also shown in Figure 3.3 is the Medium Angle Silicon Calorimeter (MASC). This calorimeter, which has not been used, was intended to extend the coverage of SLD from the lower coverage of the LAC at 200 mRad to 68 mrad. Figure 3.4 shows one of the 23 silicon layers in the LMSAT as seen from the interaction point. The octagonal shaped layer is made up from eight segments each of 20 silicon wafers.

The Bhabha event selection makes use of the narrowness of electromagnetic showers, as well as the fact that the two-body final state will be collinear. The systematic uncertainty is $\pm 1 \%$ with $\pm 0.88 \%$ from background contamination and event selection procedure, and $\pm 0.5 \%$ from theoretical uncertainty in the Bhabha cross-section. Table 3.1 lists the luminosity results for the separate SLD runs and combined results for the two periods with different vertex detectors installed. 


\subsection{The Vertex Detectors}

\subsection{The Vertex Detectors}

At the heart of the SLD, wrapped immediately around the beam pipe at the interaction point (IP), is the SLD silicon vertex detector [55].

After the invention of the charge-coupled device (CCD) in 1970, the first paper to explore the possibility of using such devices as high precision detectors of minimumionizing particles appeared in 1981 [56]. It was already then realized that CCD's offered the possibility of extraordinary physics performance in the $e^{+} e^{-}$linear collider environment, superior to what would be achievable with silicon microstrips detectors. The very small beam spots create an environment for a possible high precision vertexing if the detector has a small inner radius. Due to high background per bunch crossing, likely to be found in a linear collider, a coarsely segmented device, such as silicon microstrips, would need the inner barrel to be pushed out to much larger radius, with consequent degradation in the performance. A highly segmented pixel structure would comfortably absorb high backgrounds and with a long interval between bunch crossings the integrated time for each readout would be only $\sim 10$ bunch crossings $(80 \mathrm{~ms})$. With a typical trigger rate of $0.3-1.0 \mathrm{~Hz}$, the random coincidence rate for hits from overlapping triggers is extremely low.

In practice, the majority of tracks are generally rather low in momentum, so 


\section{The SLD Detector}

that the precision is limited by scattering in the material of the vertex detector and the beampipe, rather than by its intrinsic resolution. In these circumstances, the usefulness of the detector for physics is dependent on achieving a small inner radius, a spacing between layers similar to that (allowing a good lever-arm for extrapolation to the IP) and a smallest possible layer thickness.

\subsubsection{VXD2}

After tests with a prototype detector VXD1, consisting of a few ladders, the 120 Mpixel detector VXD2 was installed for physics runs starting in January 1992. With an intrinsic $\sim 5$ micron resolution, built out of 480 individual $9 \mathrm{~mm}$ by $13 \mathrm{~mm}$ CCD wafers with $(22 \mu \mathrm{m})^{2}$ pixel size, the VXD2 consisted of 60 ladders ( 8 CCDs per ladder) arranged in four radial layers to provide position measurements from a radius of $3.0 \mathrm{~cm}$ to $4.2 \mathrm{~cm}$ from the incoming beam axis. In VXD2, compromises were forced on the detector design due to the background conditions and the design of the off-the-shelf CCDs. The resulting design gave $50 \%$ azimuthal coverage for each of the closely packed layers and a polar angle coverage of $|\cos \theta|<0.75$.

In VXD2 a typical track would hit two (on average $2.3 \mathrm{CCDs}$ ) of the four radial layers with sometimes a radial separation between hits of only $4 \mathrm{~mm}$. The space-point precision on each hit was approximately $5.4 \mu \mathrm{m}$, but due to the poor 


\subsection{The Vertex Detectors}

VXD2

VXD3
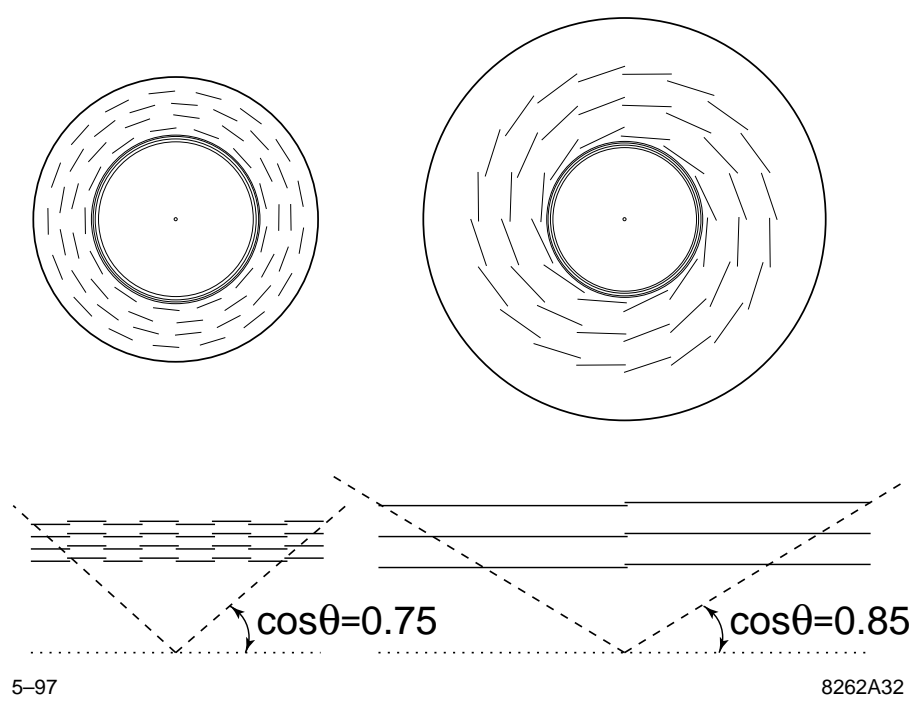

Figure 3.5: The radial layout of SLD Vertex Detectors is shown.

lever-arm the resulting precision $\sigma$ on the impact parameter at the SLD IP was strongly degraded. The precision is inversely proportional to the lever-arm, inversely proportional to the momentum and proportional to the square root of the thickness of the layers. The layer thickness for VXD2 corresponds to 0.012 radiation lengths per layer. The resulting $r-\phi$ and $r-z$ impact parameter resolution can be approximated by

$$
\begin{aligned}
\sigma_{r \phi} & =11 \oplus \frac{70}{p \sin ^{\frac{3}{2}} \theta} \mu \mathrm{m} \\
\sigma_{r z} & =38 \oplus \frac{70}{p \sin ^{\frac{3}{2}} \theta} \mu \mathrm{m},
\end{aligned}
$$

for a track of momentum $p \mathrm{GeV}$ and polar angle $\theta$ w.r.t. the $z$-axis (positron beam 


\section{The SLD Detector}

direction), $\oplus$ reflects that the individual terms are uncorrelated and that they must be added in quadrature. In Figure 3.6 the resolution parameters are plotted versus the track momentum seperately for VXD2 and VXD3 (to be described below). These numbers are similar to that of the best silicon micro strip vertex detector system at LEP (Delphi). Nevertheless, SLD has benefited from two major advantages with respect to LEP. The sub-micron beam spot size and stability in the $r \phi$ view implies that one can determine the interaction point IP extremely well by averaging the fitted IP over the last 20 interactions. Further, the very fine segmentation of the CCD detector (2500 pixels per $\mathrm{mm}^{2}$ ) means that, despite the high hit density from backgrounds and particles in a jet, the level of cluster merging is truly negligible.

As a result, the understanding of the impact parameter distribution agrees well between Monte Carlo and data over four orders of magnitude, paving the way for an elaborate interplay between data and Monte Carlo.

\subsubsection{VXD3}

As a result of rapid advances in CCD technology over the past 10 years, fueled in part by the multi-disciplinary user base, the opportunity arose for replacing VXD2 with a much more powerful vertex detector. By 1993, a great advance was the availability of affordable fully customized devices, allowing the user to tailor the 


\subsection{The Vertex Detectors}
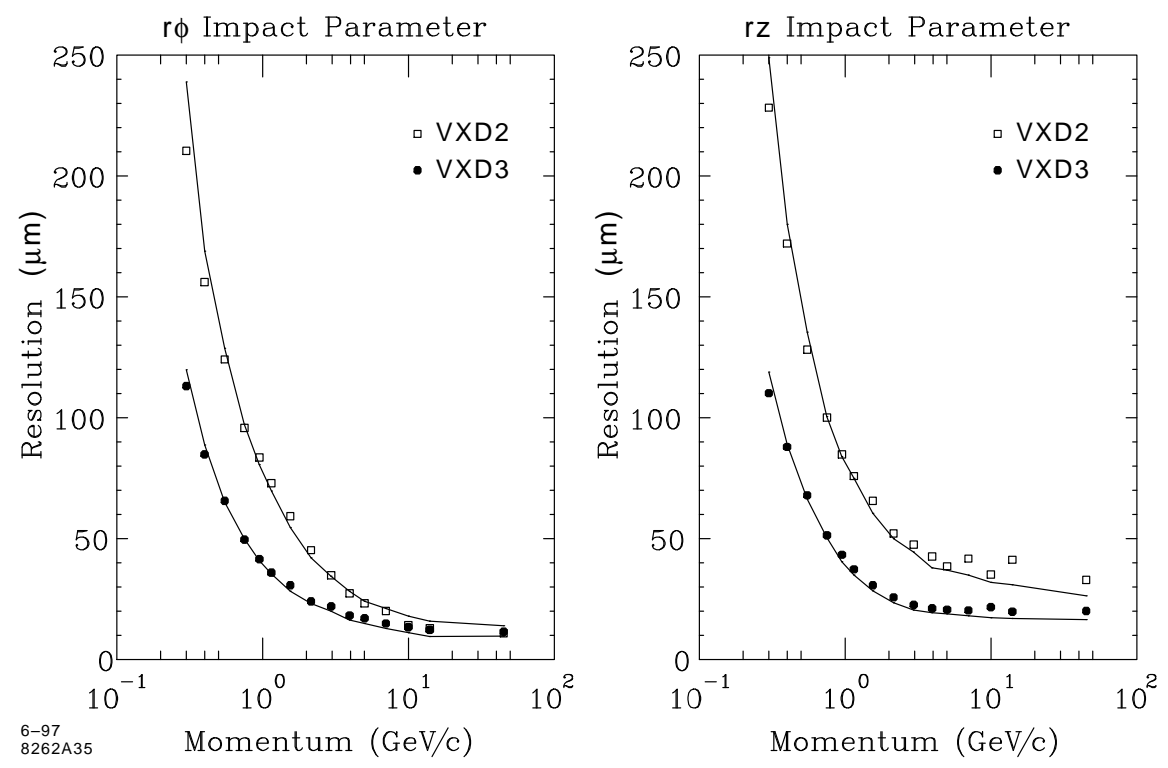

Figure 3.6: The track impact resolution as a function of momentum. The results for the VXD2 and the VXD3 detectors are shown separately.

design to the specific detector requirements. Furthermore, an important advance was the availability of much larger scientific grade devices of 5 inch diameter wafers, reflecting an enormous progress in quality control on the part of the manufactures, allowing for the transport of signals of a few hundred electrons reliably across more than 2000 pixels.

These improvements allowed for full flexibility regarding the layout of circuitry around the imaging area and it became possible to eliminate large inactive regions. Improved readout techniques allowed for an order of magnitude increase in the active area without any increase in readout time. The proposal to build this upgraded 


\section{The SLD Detector}

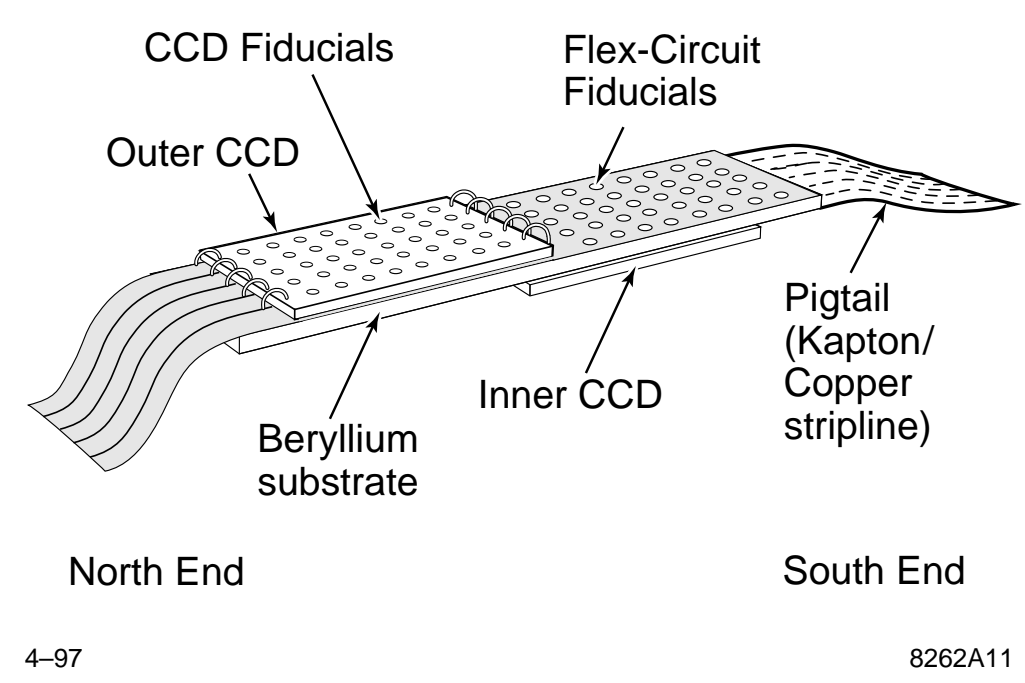

Figure 3.7: A closeup of a single VXD3 ladder showing the two CCDs.

detector VXD3 was approved in March 1994, and it was installed in January 1996, just days before the startup of the 1996 run.

The 307 Mpixel VXD3 detector has three active overlapping layers, each with a number of beryllium ladders that lie parallel to the beam direction, and provide position measurements from a radius of $2.8 \mathrm{~cm}$ to $4.8 \mathrm{~cm}$. There are a total of 48 ladders which each carry 2 CCDs, one on the top (northern hemisphere) and one on the bottom (southern hemisphere). See Figure 3.7 for a display of one of the 48 VXD3 ladders. Each CCD contains $4000 \times 800$ pixels of size $(20 \mu \mathrm{m})^{2}$ each. The layer thickness is reduced to 0.004 radiation lengths, greatly reducing the effects of multiple scattering, while the active layer length along the beam axis is increased 


\subsection{The Vertex Detectors}

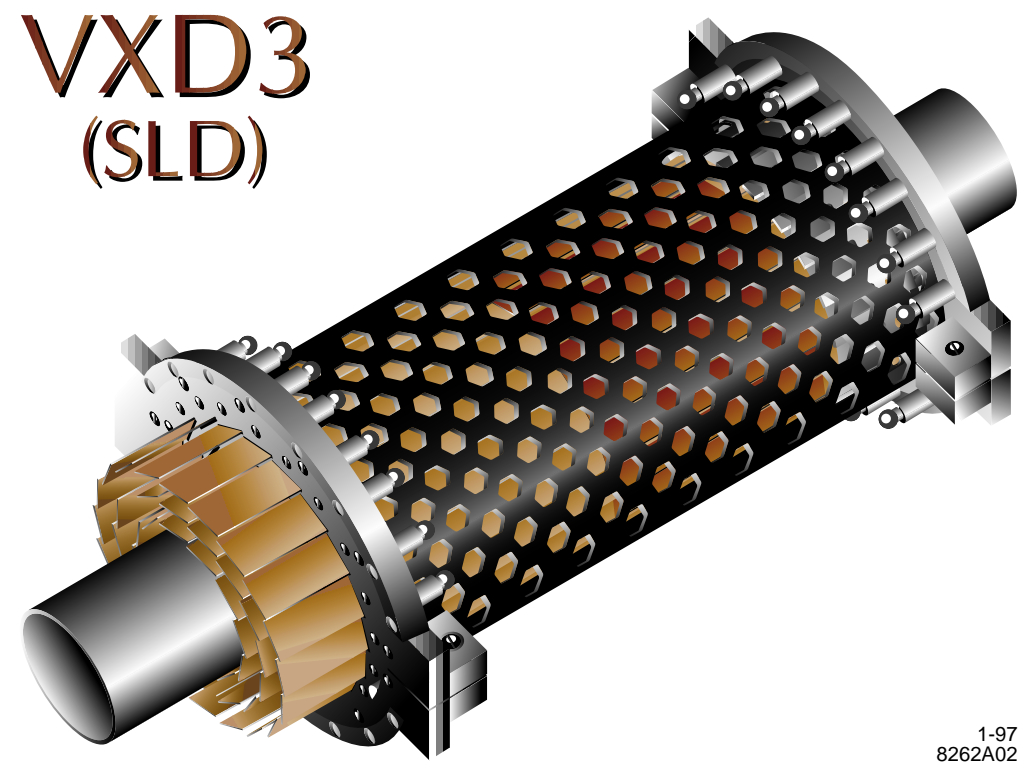

Figure 3.8: The VXD3 detector surrounding the beam line.

by a factor of 1.7 to $16 \mathrm{~cm}$ allowing angular coverage out to $|\cos \theta|=0.85$. Figure 3.8 shows VXD3 in an isometric view.

The overall impact parameter resolution of VXD3, deduced from data, is approximated by

$$
\begin{aligned}
\sigma_{r \phi} & =14.0 \oplus \frac{33}{p \sin ^{\frac{3}{2}} \theta} \mu \mathrm{m}, \\
\sigma_{r z} & =26.5 \oplus \frac{33}{p \sin ^{\frac{3}{2}} \theta} \mu \mathrm{m},
\end{aligned}
$$

and is a substantial improvement compared to VXD2 (Figure 3.6). There remains only a small discrepancy with respect to the Monte Carlo expectations mostly in the 


\section{The SLD Detector}

$r z$ view for high momentum tracks. It has been shown that this discrepancy is caused mainly by a problem with the central drift chamber tracks in the combined fit. If only VXD3 is used in the analysis, the constant term is reduced from $26.5 \mu \mathrm{m}$ to $15.9 \mu \mathrm{m}$. This problem is being fixed at the moment of writing, but unfortunately this analysis will not benefit from this improvement, and this becomes a source of systematic error for the analysis described in this thesis. For $\sigma_{r \phi}$ and the multiple scattering term ( $p$ dependent) the detector is already delivering its design performance.

\subsection{Central Drift Chamber}

Immediately surrounding the Vertex Detector is the Central Drift Chamber (CDC) which provides charge and momentum measurements for each of the charged tracks present in an event [57]. Two planar endcap drift chambers (ECDC) closes the cylindrical CDC to extend the reach of the SLD tracking, however the analysis of the data from these chambers has proven to be difficult due to large beam-induced backgrounds and will not be considered further. The CDC covers roughly $80 \%$ of the $4 \pi$ solid angle and operates in a uniform solenoidal magnetic field of 0.6 Tesla. Its shape is that of a cylindrical annulus. It is $2 \mathrm{~m}$ long with its inner radius at 20 $\mathrm{cm}$ while the outer radius is $100 \mathrm{~cm}$. The CDC has 10 superlayers consisting of 640 


\subsection{Central Drift Chamber}

drift cells each. Each drift cell is sensitive to a volume of roughly $6 \mathrm{~cm}$ width and 5 $\mathrm{cm}$ height in cross section along the entire $2 \mathrm{~m}$ length of the chamber, to measure the curvature of a charged track, and hence its momentum, as it passes through the 0.6 Tesla solenoidal magnetic field provided by the SLD's $6.6 \mathrm{kAmp}, 5.0$ MWatt superconductive magnetic coil. The superlayers are staggered in orientation, with the drift cells oriented along the cylinder axis for the axial superlayers and with the drift cells tilted by an angle of $41 \mathrm{mrad}$ in the stereo superlayers (Figure 3.9), providing longitudinal as well as axial information.

Each drift cell contains field wires that define a nearly constant electric field across each cell. See Figure 3.10 for the layout of the drift cells. A charged particle passing through the drift chamber will ionize the drift chamber gas along its path, depositing on the order of $5 \mathrm{keV} / \mathrm{cm}$ for a minimum ionizing track. The charge liberated in the ionization process drifts with a uniform velocity of 8 microns/ns within the $0.9 \mathrm{kV} / \mathrm{cm}$ field towards a plane of the sense wires. Immediately on either side of the sense wires are guard wires which define the boundary of the drift cell. A voltage difference of $3 \mathrm{kV}$ between the guard and sense wires provides some signal amplification as the individual electrons avalanche down onto the sense wires. The CDC gas is a mixture of $75 \%$ carbon dioxide, $21 \%$ Argon, $4 \%$ isobutane, and $0.3 \%$ water chosen to provide good ionization properties and drift velocity, while 


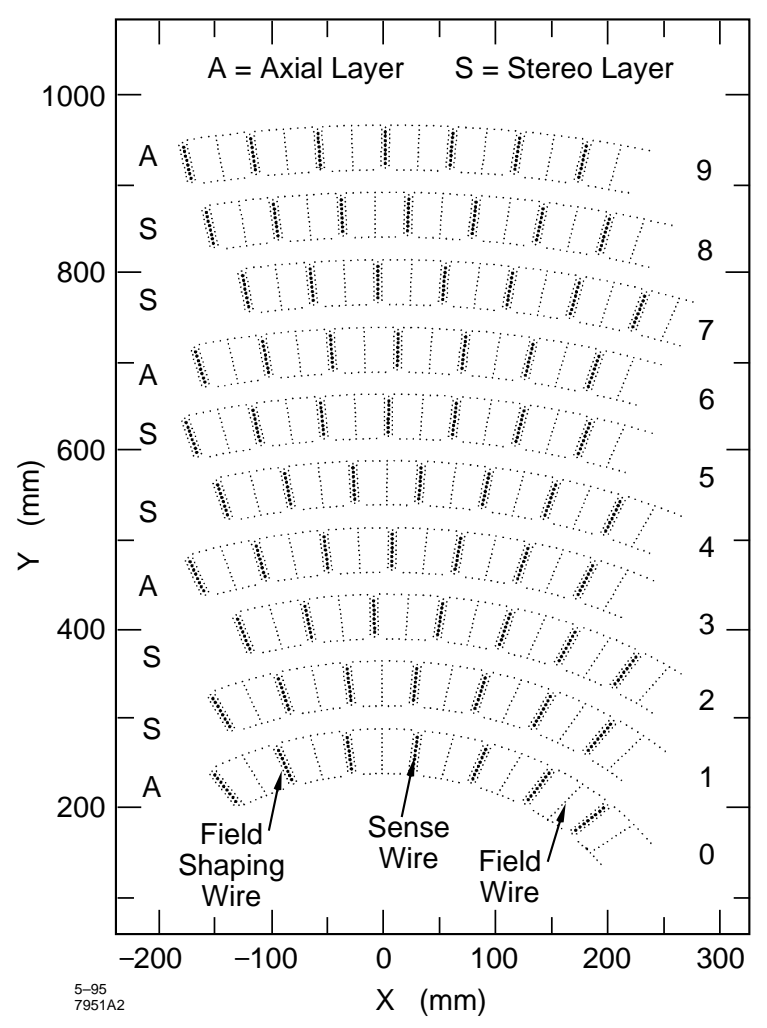

Figure 3.9: The CDC superlayers are shown with the belonging drift cells. The Field Shaping Wires are also named guard wires.

being resistant to electrical breakdown and charge diffusion. See Figure 3.11 for a map of the electric field (left figure) and the trajectory of the electrons from an ionizing particle (right figure).

The electrical pulse resulting from the charge deposited on both ends of each sense wire is sampled at $119 \mathrm{MHz}$ and stored in a 512 channel switched capacitor array. A discriminated charge sum for each wire is also provided on every beam crossing to the FASTBUS based trigger logic algorithm. For triggered events, these waveforms 


\subsection{Central Drift Chamber}

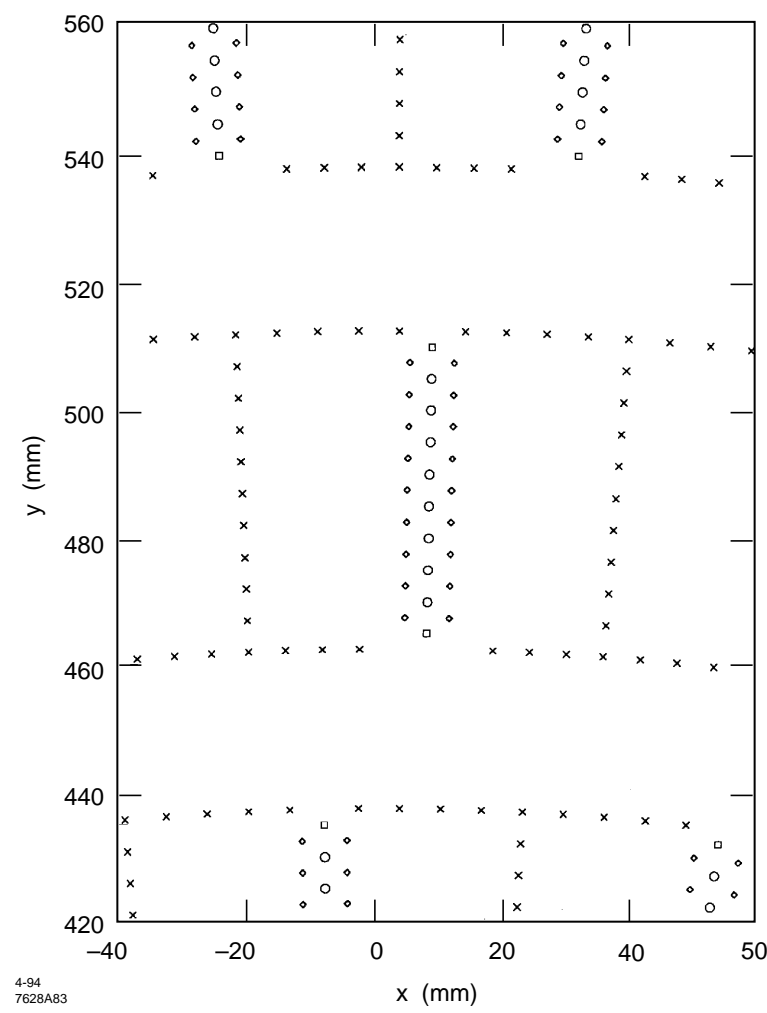

Figure 3.10: A closeup of a single drift cell in the CDC is shown. Voltage applied to the field wires (crosses) defines a nearly constant electric field running the length of the cell towards the guard wires (diamonds). Only the eight sense wires (circles) are instrumented for readout.

are digitized by 12 bit Analog to Digital Converters (ADCs) and shipped to the FASTBUS based waveform analyzing processor (WASM). This processor calculates the time, charge, pulse height, and pulse width of the observed waveform which, when combined with the known drift time of the gas and detector geometry, allows a track position in space to be reconstructed. Double hits observed on a single wire can be resolved with reasonable efficiency down to a transverse track separation of 1 


\section{The SLD Detector}
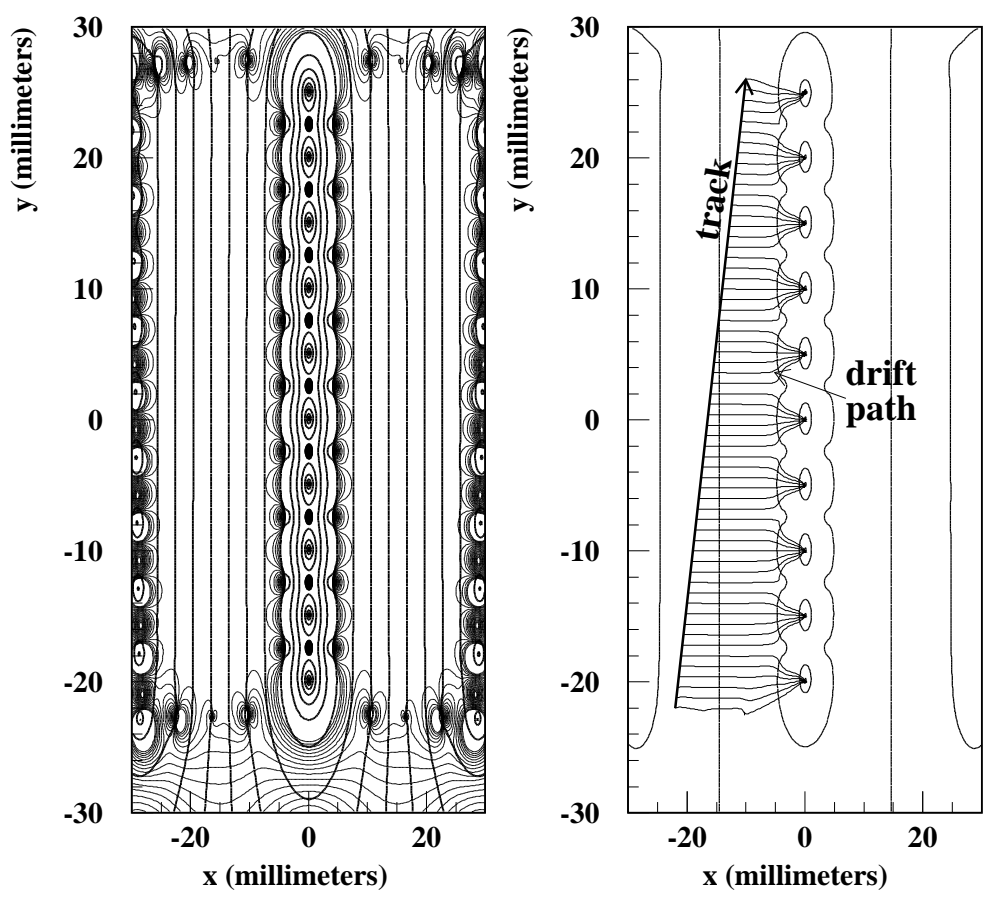

Figure 3.11: The field map for a drift cell of the CDC. The left figure shows lines of constant potential (bold) and lines of constant field strength (thin) within a CDC cell. The right figure shows a drift path of charges caused by the passing of a charge track through the cell.

$\mathrm{mm}$. The CDC data acquisition requires $80 \mathrm{~ms}$ (10 beam crossings) to fully digitize, read out, and analyze the CDC waveforms. Due primarily to budgetary constraints, multiple CDC events cannot be buffered, and during this readout time the CDC electronics are insensitive to any new incoming data.

The transverse distance of a track from an individual sense wire is measured to an intrinsic resolution of approximately 70 microns in each cell, although uncertainties 


\subsection{Central Drift Chamber}

in the wire locations and changes in the drift velocity degrade this to an effective resolution of around 100 microns. While the longitudinal location of a track can be roughly estimated to a few centimeters by the amount of current produced at either end of the highly resistive tungsten wires, a more precise determination is made by combining the data from several layers, including stereo information.

\subsubsection{Track Reconstruction}

Track reconstruction is accomplished via four steps in SLD. The first of these steps is to find the raw hits. The double-ended readout enables the $z$ position of each hit along the sense wire to be estimated by calculating the asymmetry of the charge division of the pulses, while the drift time information can be used to determine the distance of the hit from the sense wire via the time to distance relationship ${ }^{1}$. Certain thresholds were set to discriminate the noise from the signal pulse.

The second step is to use hits within a cell to form into track segments, usually called vector hits (VH). There must be at least three hits present in a cell to form a track segment, which is characterized by a space point $\vec{x}$ and a two dimensional direction $\vec{t}$ in the $x-y$ plane. The hit positions are then corrected for the effects of

\footnotetext{
${ }^{1}$ For each hit in a drift cell, the elapsed time between a beamcrossing to the time when the hit is monitored, is used for calculating the radial distance of a hit to the sense wire.
} 


\section{The SLD Detector}

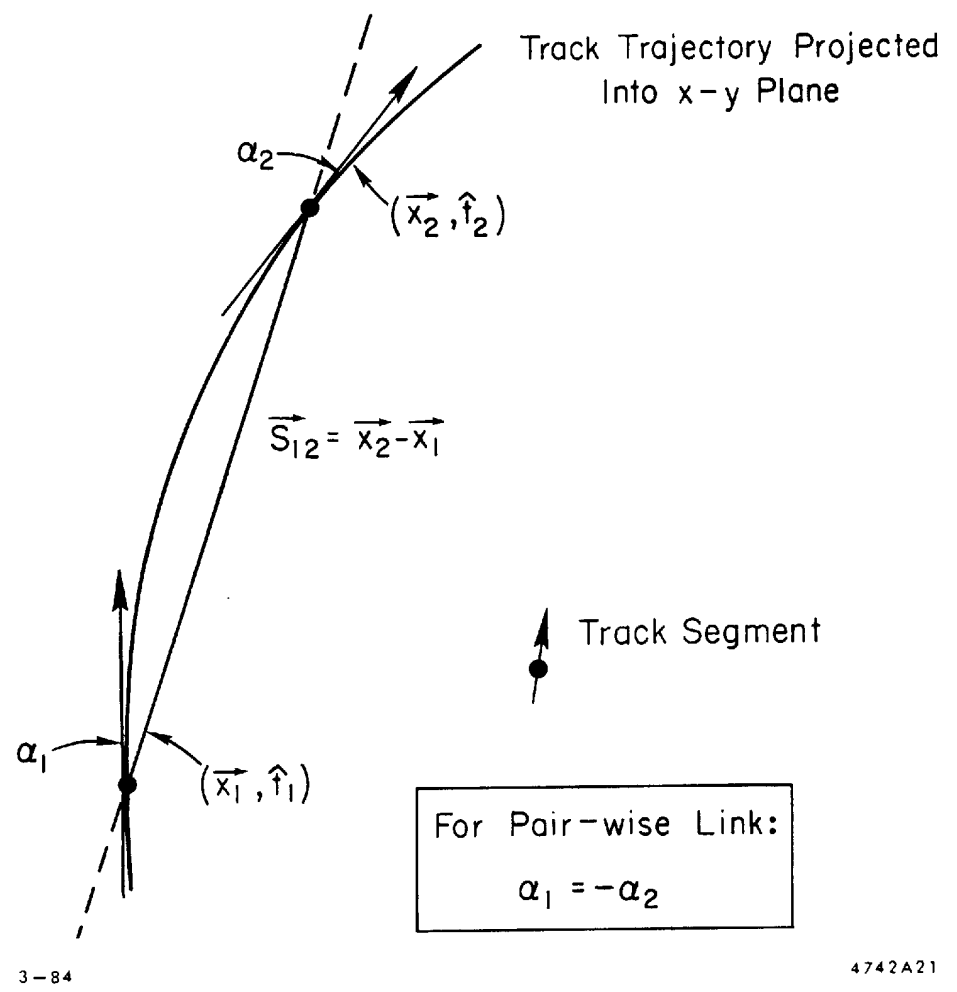

Figure 3.12: This shows the method for linking track segments by using the opposite angles of two segments relative to the straight line in between them.

the relative angle between the track segment and the sense wire plane through the time to distance relationship as mentioned above.

The third step is to link these track segments together, a process also called pattern recognition. The linking algorithm operates in the $x-y$ plane, as shown in Figure 3.12. If two track segments belong to the same track then they must have equal (but opposite-signed) angles with respect to the vector joining the spatial coordinates of the track segments. Initially, the combination of VHs on axial layers 


\subsection{Central Drift Chamber}

are formed by fitting them into circles. The VHs from stereo layers are then added if they fit on these circles. The $z$ information from the charge division measurement is used to project the stereo VHs onto the circles. In the first instance, only tracks with $10 \mathrm{VHs}$ are considered, and the one with the best $\chi^{2}$ is taken as a candidate track. Its VHs are removed from further consideration, and the process is repeated until all tracks of at least three VHs are found.

The last step is to process all track candidates by an iterative track fitter. The fitter starts with the estimated track parameters from the pattern recognition. It then swims a helical trajectory through the detector material, taking into account the variation of the magnetic field, energy loss and multiple scattering. The fitter uses the individual hits of the candidate tracks, and may add or delete hits as the iterations proceed. Finally, a best set of helix parameters describing the track is obtained.

A track passing through all CDC layers is ideally expected to have a hit in each wire layer, thus making 80 hits in total. Shown in Figure 3.13 is the distribution of the number of hits found on a track with a comparison to MC. Figure 3.14 shows the hit-finding efficiency as a function of wire layer, where layer 0 is the innermost layer. The lower efficiency on the inner layers is attributed to the finite two-hit resolution as the tracks become closer, and the higher backgrounds in this region. Figure 3.15 


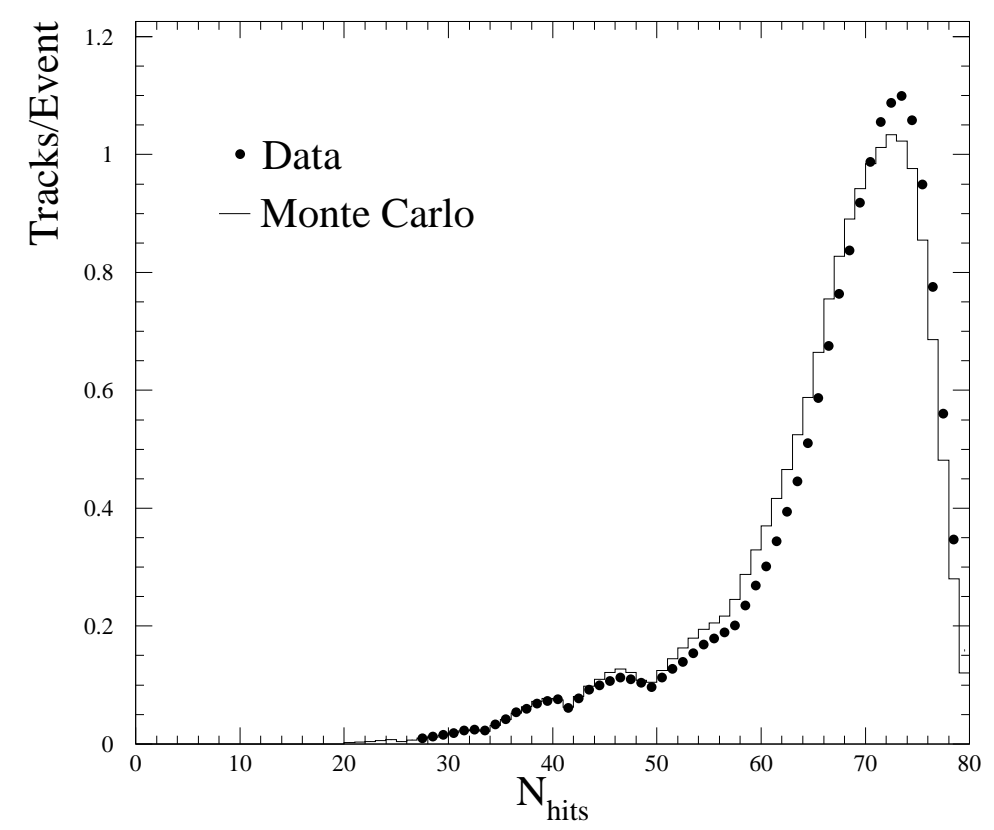

Figure 3.13: Distribution of number of hits on track.

shows the drift distance resolution measured as a function of the drift distance. A distinction is made between local and global resolutions. The local resolution is determined from the width of the distribution of the residuals to the vector hits in each cell, while the global resolution is determined from the width of the distribution of the residuals to the fitted track. In the region of linear field the resolution follows the curve expected from diffusion $(68 \mu \mathrm{m}$ at $1 \mathrm{~cm}$ and varying as $\sqrt{d})$. As it is seen, 


\subsection{Central Drift Chamber}

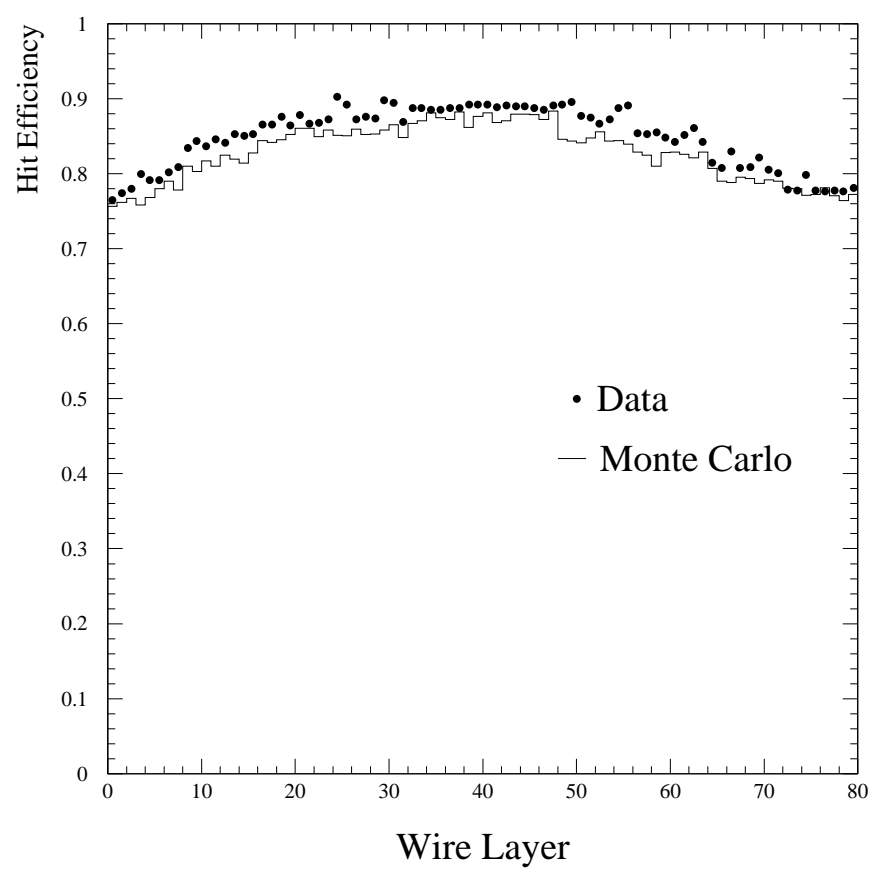

Figure 3.14: Hit-finding efficiency as a function of wire layer.

the spatial resolution has a strong dependence on the distance to the sense wire. Note the degradation of the resolution in the regions near the sense wire and field wires. This is mainly due to the increasing drift velocity and non-uniformity of the drift fields in these regions.

The relative momentum resolution provided by the CDC alone has been measured to be $\sigma_{p} / p=0.0095 \oplus 0.0049 p$, while the relative resolution of the combined CDC and 


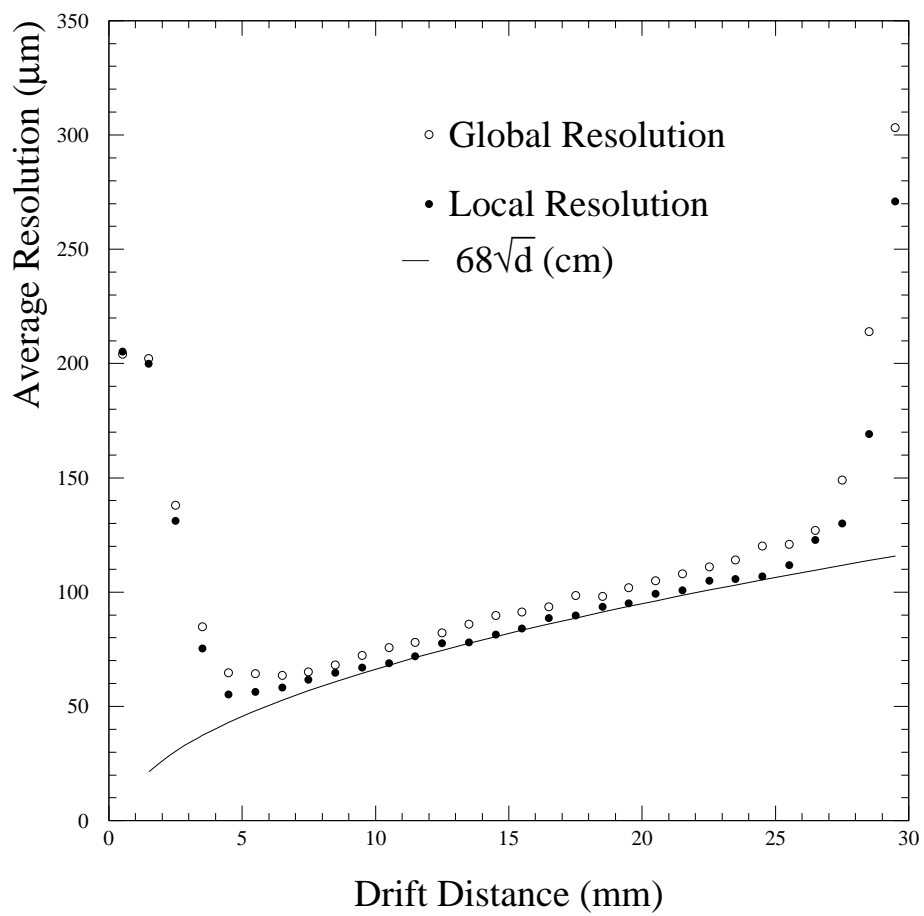

Figure 3.15: Drift distance resolution in CDC measured as a function of the drift distance.

VXD systems has been measured to be $\sigma_{p} / p=0.0095 \oplus 0.0026 p[58]$ for VXD. The first term is due to multiple scattering and the added information from the vertex detector does not improve this resolution ${ }^{2}$. For $|\cos \theta|<0.65$, the CDC can detect tracks with a uniform $96 \%$ efficiency with a momentum above $4 \mathrm{GeV}$, degrading only to $93 \%$ at $100 \mathrm{MeV}$. The geometry of the CDC provides uniform acceptance

\footnotetext{
${ }^{2}$ For high momentum tracks the vertex detector is used to constrain the parameters from the reconstructed tracks in the $\mathrm{CDC}$ by providing high precision impact parameters. In the case when multiple scattering occurs between the vertex detector and the drift chamber, such added information is less beneficial due to the unknown path of the track at the boundary.
} 


\subsection{Central Drift Chamber}

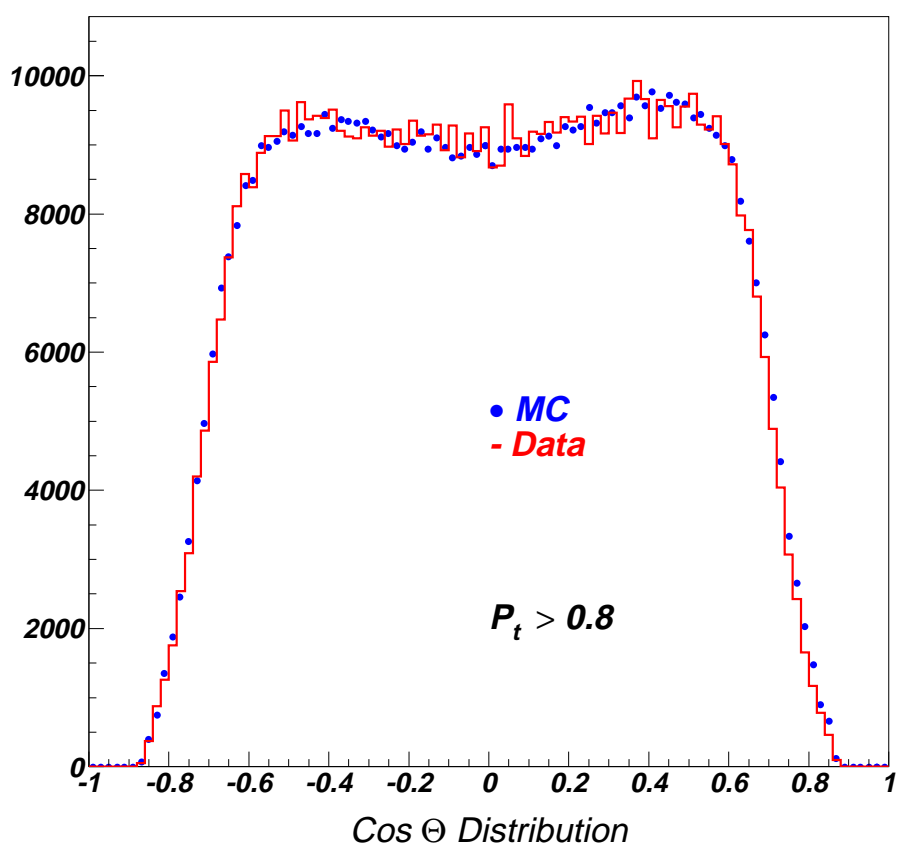

Figure 3.16: The $\cos \theta$ distribution is shown for the reconstructed charged tracks in the $R_{b} B$ sample for both $\mathrm{MC}$ and data. Tracks with transverse momentum less than $0.8 \mathrm{GeV}$ is not included in the distribution.

out to a production angle of $|\cos \theta|<0.65$, and some tracks can be reconstructed out to a production angle of $|\cos \theta|<0.87$. Figure 3.16 shows the $\cos \theta$ distribution for all reconstructed charged tracks above $800 \mathrm{MeV}$ in the $B$-meson events used in the $R_{b}$ analysis.

Drift chambers are somewhat sensitive devices in that excessive charge deposition can weaken and eventually break the fine sense wires. This would be a catastrophic 
The SLD Detector

failure in a chamber the size of the CDC, and some care is taken not to apply voltage to the chamber when SLC beam conditions are particularly bad. For this reason, the CDC was turned on for only 95\% of the 1993-98 SLC running period ${ }^{3}$.

\section{4 Čerenkov Ring Imaging Detector}

Particle identification is one of the most challenging experimental problems in any particle physics experiment. The high momentum tracks produced in $Z$ boson decays are particularly ill suited to conventional techniques which measure the energy loss $(d E / d x)$ or time of flight of an observed track in an attempt to identify the particle type through an estimate of its mass. Situated just outside of the CDC, the Čerenkov Ring Imaging Detector (CRID) identifies particles by observing the cone of Čerenkov light ${ }^{4}$ produced by the track as it traverses either a gas or liquid radiating medium [59]. The radius of this cone of Čerenkov light is used to extract the track velocity, which together with a matching momentum information from the central drift chamber provides information of the particle mass, thus identifying the

\footnotetext{
${ }^{3}$ In September 1997 the experiment was halted for a period of a month when the location and subsequent removale of a broken wire took place.

${ }^{4}$ Particles traveling faster than the speed of light in a given medium emit a shock wave of Cerenkov photons with an opening angle $\cos \theta_{c}=1 / n \beta$ for index of refraction $n$ and particle speed $\beta=v / c$. The threshold velocity $\beta_{\text {threshold }}=1 / n$. The index of refraction is 1.2780 and 1.0026 for the liquid and the gas radiator respectively.
} 


\section{4 Čerenkov Ring Imaging Detector}

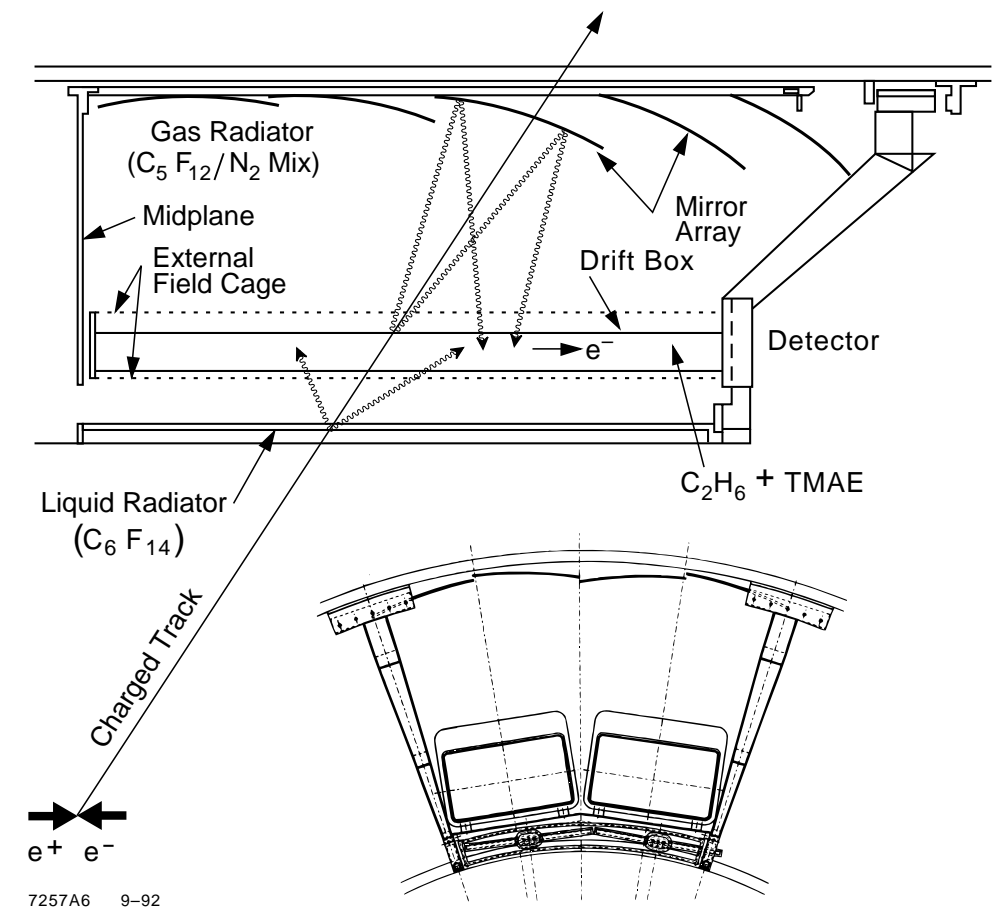

Figure 3.17: The geometry of a single CRID module is shown. In the upper plot (axial view), photoelectrons from both the liquid and gas radiator are detected on opposite sides of the same TPC. In the lower plot (radial view) the two TPC's in each sector is shown as the boxes.

particle species.

In order to extend the useful momentum range of the device, two radiating materials are used. A liquid radiator composed of $\mathrm{C}_{6} \mathrm{~F}_{14}$ with an index of refraction of 1.2780 provides good $\pi / K / p$ particle identification in the momentum range from 0.5 to $3.0 \mathrm{GeV}$, while a gas radiator composed of $\mathrm{C}_{5} \mathrm{~F}_{12}$ gas with an index of refraction of 1.0026 covers the higher momentum range up to $35 \mathrm{GeV}[60]$.

The CRID mechanical layout structure observes the general SLD partition into 


\section{The SLD Detector}

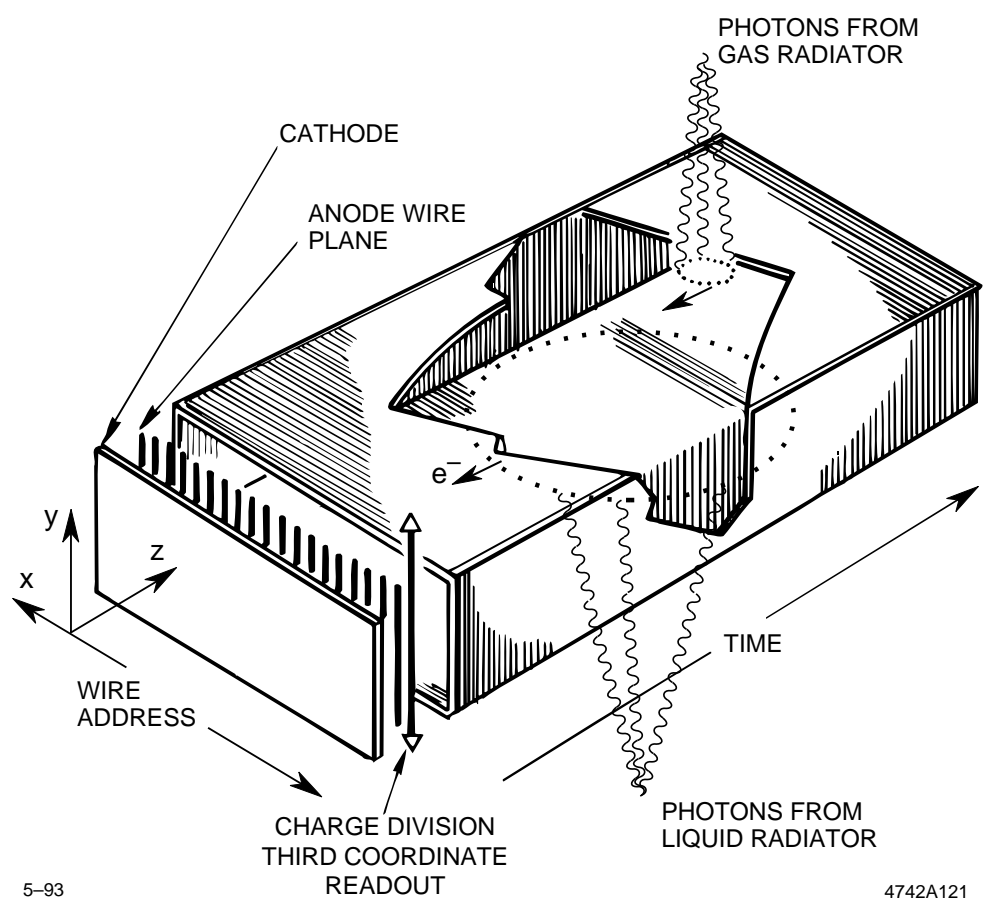

Figure 3.18: Schematic view of a TPC and MWPC detector for the barrel CRID. Also shown is the standard TPC coordinate system employed in the CRID reconstruction.

a Barrel and a End-Cap system. The Barrel CRID is segmented into 20 sectors, 10 each for the south (negative $z$ direction) and north (positive $z$ direction) side of the detector. Each sector consists of two liquid Čerenkov radiator trays, two TPC boxes (which convert Č herenkov photons into electrons via the photoelectric effect), and 20 mirrors. A gaseous radiator vessel embeds all 20 sectors. The two back-to-back 1.2 meter long drift boxes in the barrel, provide particle ID coverage out to a production angle of $|\cos \theta|<0.72$ which is fairly well matched to the CDC tracking coverage. 


\section{4 Čerenkov Ring Imaging Detector}

As shown in Figure 3.17, the Čerenkov photons from both radiators are detected in a time proportional chamber (TPC) which is essentially just a long drift chamber. Each quartz-windowed TPC cell is filled with an ethane drift gas doped with a $0.1 \%$ concentration of the photo reactive substance tetrakis (dimethylamino) ethylene (TMAE), which provides good quantum efficiency for converting Cerenkov photons into electrons. These electrons drift the length of the TPC and are detected by multi-wire proportional chamber (MWPC) detectors located at the outer end of the TPCs. A sketch of the TPC - MWPC setup is shown in Figure 3.18.

A typical track will have on the order of 10 detected photoelectrons from which to reconstruct a ring radius and make a particle identification, although the high quantum efficiency of the TMAE doped gas makes these chambers extremely sensitive to beam-related backgrounds. The possibility of high backgrounds breaking CRID sense wires is a constant concern during SLD operations, and while not catastrophic in the same sense that breaking a CDC wire would be, it does put that particular CRID module out of commission until it can be fixed. This was observed to happen a couple of times during the 1994-98 running period, and to protect the device it was typically turned off during periods of particularly bad backgrounds. For this reason, CRID data is only available for $80 \%$ of all data taken with the drift chamber on. The CRID data has a comparable readout time as that of the CDC. 


\section{The SLD Detector}

The endcap CRID modules, sandwiched between the endcap drift chambers, were designed to extend the particle ID capabilities of the SLD into the forward region. Unfortunately, without a mature endcap tracking system these chambers are largely unusable, and will not be considered in this analysis.

\subsection{Liquid Argon Calorimeter}

Located just inside the SLD coil from $1.8 \mathrm{~m}$ to $2.9 \mathrm{~m}$ of radius in the barrel, the Liquid Argon Calorimeter (LAC) is a sampling calorimeter which provides an energy measurement of neutral and charged hadronic as well as electromagnetic particles [61]. A calorimeter works by putting enough radiating material in the way of an incoming particle to stop that particle through interactions and energy loss. In a sampling calorimeter, instrumented layers of some ionizing medium are interspersed with the radiator material to sample the energy deposited as a function of shower depth. The sum of this sampled energy is proportional to the total energy of the incident particle.

The choice of liquid argon and lead was made to provide the most cost effective large area coverage with uniform energy response, good radiation resistance, and fine spatial segmentation. 


\subsection{Liquid Argon Calorimeter}

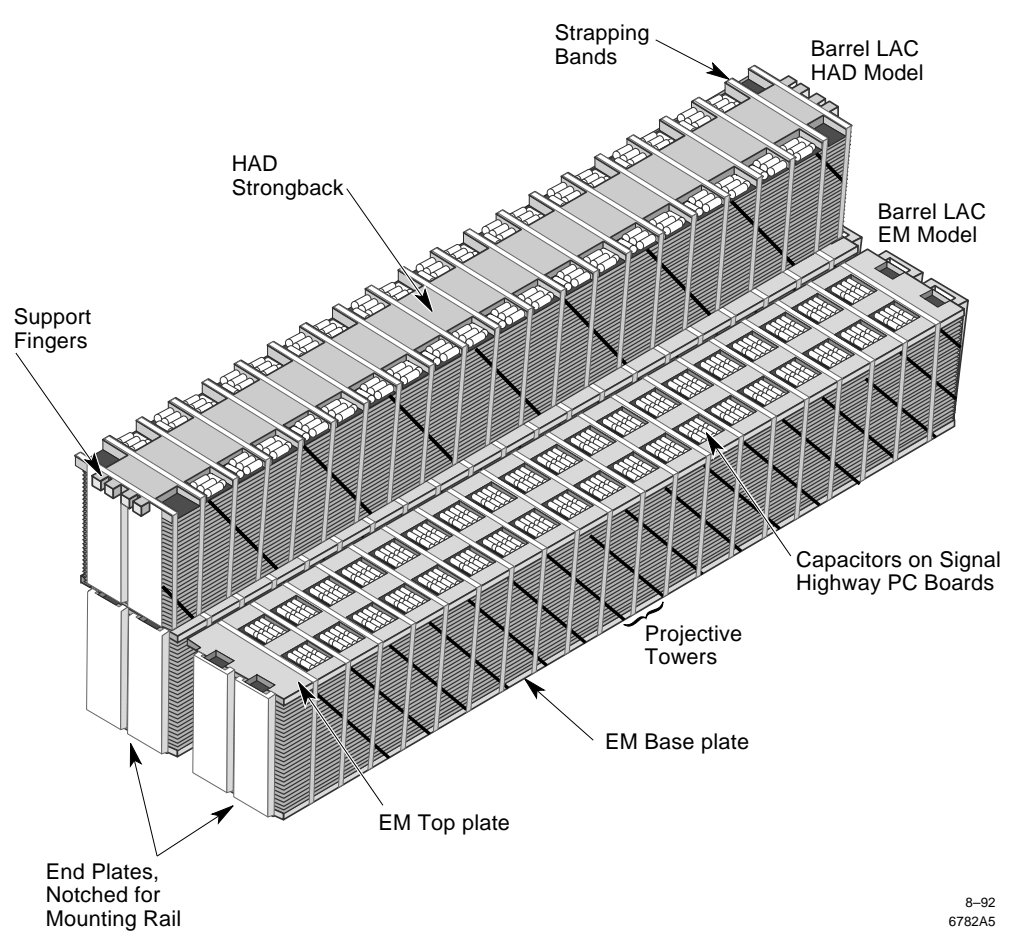

Figure 3.19: The geometry of a LAC module is shown. One HAD module is shown on top of two EM modules. The longitudinal segmentation of the Lead-Argon sandwich is ganged together into four logical readout layers to reduce the total number of detector channels.

The LAC is composed of a cylindrical barrel calorimeter and two endcap calorimeters, each housed in a separate cryostat containing a combined 35,000 liters of liquid argon. The barrel section covers the region $|\cos \theta|<0.84$ and is composed of 288 modules. In the radial direction (as seen from the beam line), two separate modules are mounted on top of each other, the electromagnetic (EM) modules and hadronic (HAD) modules (Figure 3.19). The endcap calorimeters cover the region $0.82<|\cos \theta|<0.99$ and are made from 16 wedge-shaped modules, each module also 
The SLD Detector

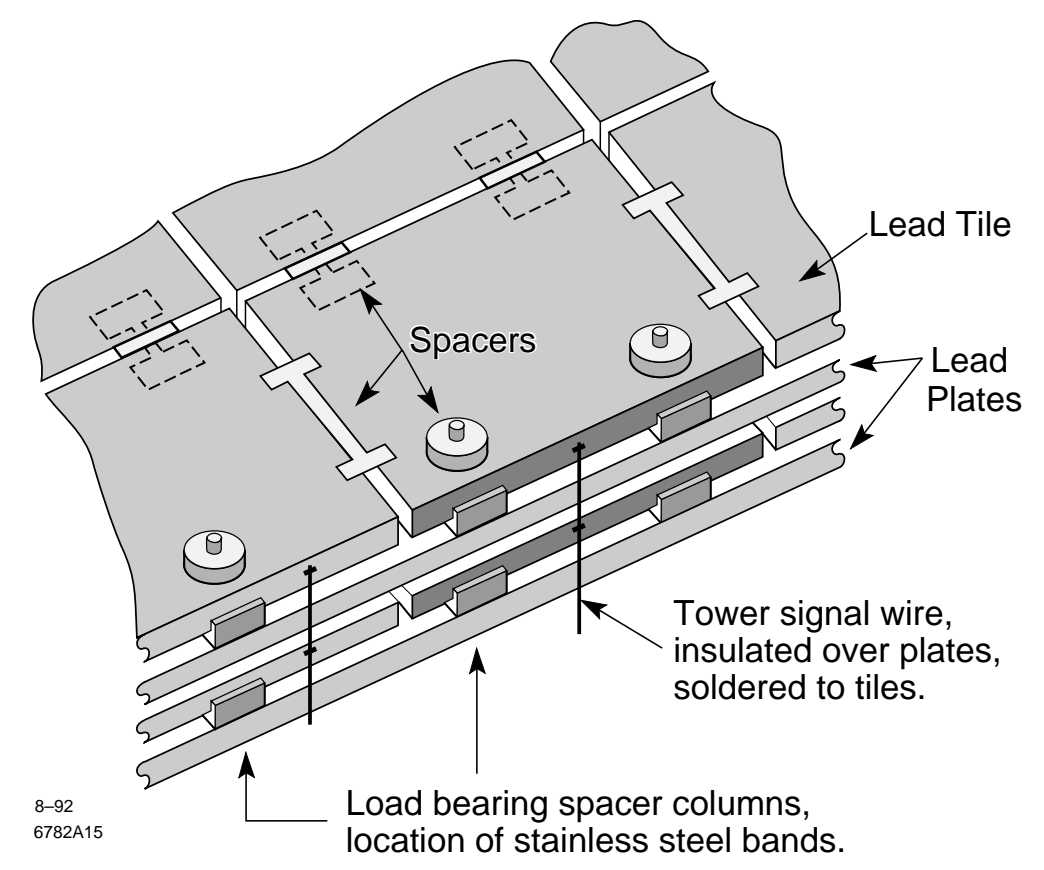

Figure 3.20: The arrangement of lead sheets and tiles which make up the LAC are shown. Voltage is applied to the lead sheets and signals are read out on the lead tiles. The geometry of the individual tiles defines the transverse segmentation of the LAC.

containing an EM and HAD section. Endcap modules differ from barrel modules in the geometry and layout, but are functionally identical (Figure 3.21).

Each LAC module is constructed out of lead sheets and tiles as shown in Figure 3.20. The innermost module consists of 56 lead plates, each $2 \mathrm{~mm}$ thick, spaced $2.75 \mathrm{~mm}$ apart to provide space for the liquid argon to fill the active layer. This electromagnetic (EM) section provides a total of 21 radiation lengths of material which will absorb $98 \%$ of the energy from a $50 \mathrm{GeV}$ electron. High voltage $(\sim 2000$ 


\subsection{Liquid Argon Calorimeter}

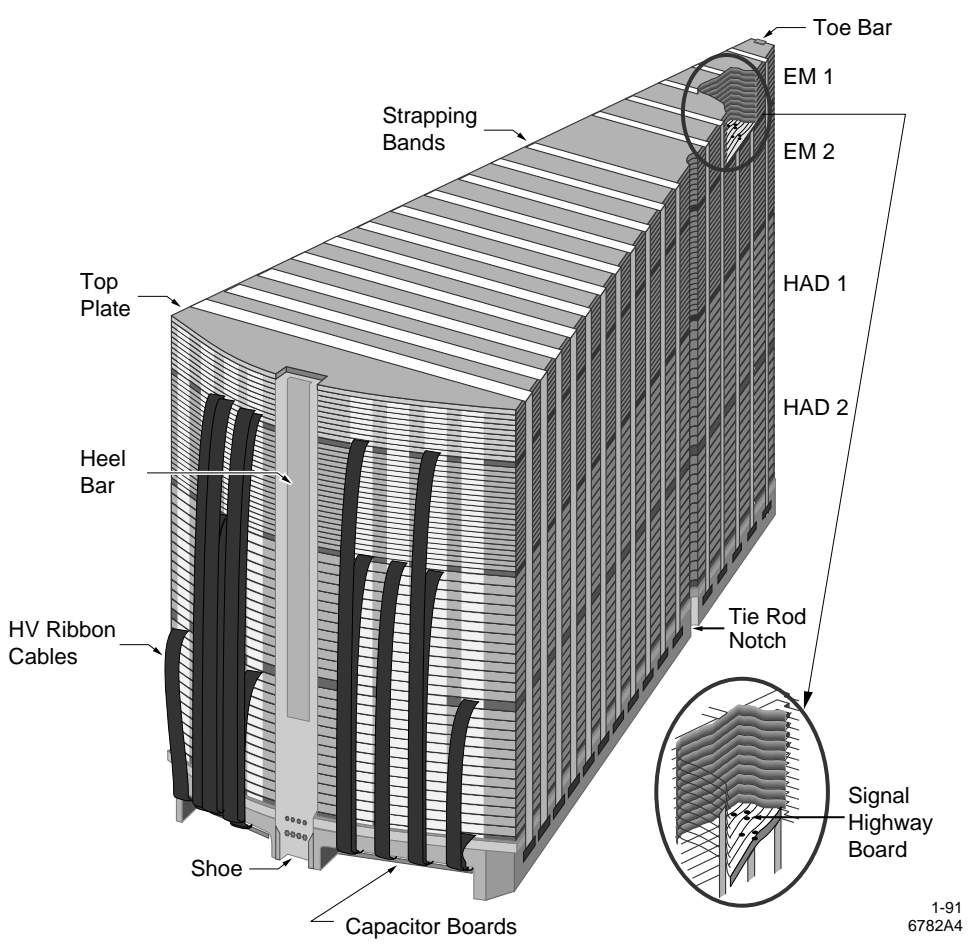

Figure 3.21: View of a LAC endcap module, showing the inner EM section (top) and the outer HAD section (bottom).

V) applied between the lead sheets and tiles collects the ionization from the liquid argon onto the lead tiles which are electrically connected to form a single readout layer. In the EM section, the first 16 and remaining 40 planes are connected in this way to provide two longitudinal readout layers of 6 radiation lengths and 15 radiation lengths respectively. The energy resolution of the EM section to either electrons or photons is measured to be $15 \% / \sqrt{E(\mathrm{GeV})}$.

The outer module consists of 26 lead plates, each $6 \mathrm{~mm}$ thick, spaced $2.75 \mathrm{~mm}$ 


\section{The SLD Detector}

apart. This hadronic (HAD) section is evenly divided into two longitudinal readout layers of 1 absorption length each, which when combined with the EM section provides a total of 2.8 absorption length of material. This calorimeter will contain around $90 \%$ of the energy from a hadronic shower, providing a hadronic energy resolution of $65 \% / \sqrt{E(\mathrm{GeV})}$.

The spatial resolution of the LAC is determined by the segmentation of the readout tiles, which are arranged in a roughly projective tower geometry. The $6 \mathrm{~m}$ long barrel LAC is divided into 96 polar and 192 azimuthal towers in the EM section with each EM tower subtending an angle of $\delta \phi=33 \mathrm{mRad}$ in azimuth and ranging from $\delta \theta=36 \mathrm{mRad}$ in polar angle at the center of the barrel to $\delta \theta=21 \mathrm{mRad}$ at the end of the barrel to maintain a constant projective area. The HAD towers match the projective geometry of the EM section, although they are more coarsely segmented by a factor of two in both the polar and azimuthal dimensions.

There are a total of 32,448 individual channels in the barrel LAC with an additional 8,640 channels in the endcap. Each channel is digitized after a dual gain charge sensitive preamplifier by a multiplexed 12 bit ADC to provide an effective 15 bit dynamic range. The digitized data, along with baseline information, is then shipped by serial fiber link to one of the 32 FASTBUS based Calorimetry Data Modules (CDMs) where the raw data is converted into a single measured energy per 


\subsection{Warm Iron Calorimeter}

channel. Energy sums are also calculated by detector region and readout layer to be used in the trigger decision. The entire LAC can be read out and analyzed in $\sim 4$ ms providing a nearly dead-time free acquisition system.

\subsection{Warm Iron Calorimeter}

Outside of the magnetic coil is the Warm Iron Calorimeter (WIC) [62]. The massive WIC structure provides muon tracking with 18 layers of Iarrochi tubes sandwiched between the $5 \mathrm{~cm}$ plates of WIC steel, some amount of additional calorimetry information, as well as a flux return path for the solenoidal magnetic field (Figure 3.22). The Iarrochi tubes used are small $1 \mathrm{~cm}$ square plastic wire chambers operated in limited streamer mode with a single wire running the length of the $6.8 \mathrm{~m}$ barrel modules. The wires themselves are not instrumented, but rather capacitive strips and pads immediately outside the Iarrochi tubes detect the current streamers produced by muons passing through the tubes. In the barrel, there are 14 layers of longitudinal strips parallel to the beam axis, and 4 layers of transverse strips used to track muons as they pass through the WIC steel. The endcap modules have a similar thickness with half of the tubes running vertically and the other half running horizontally. The WIC strips are a binary system, with each strip being discriminated 


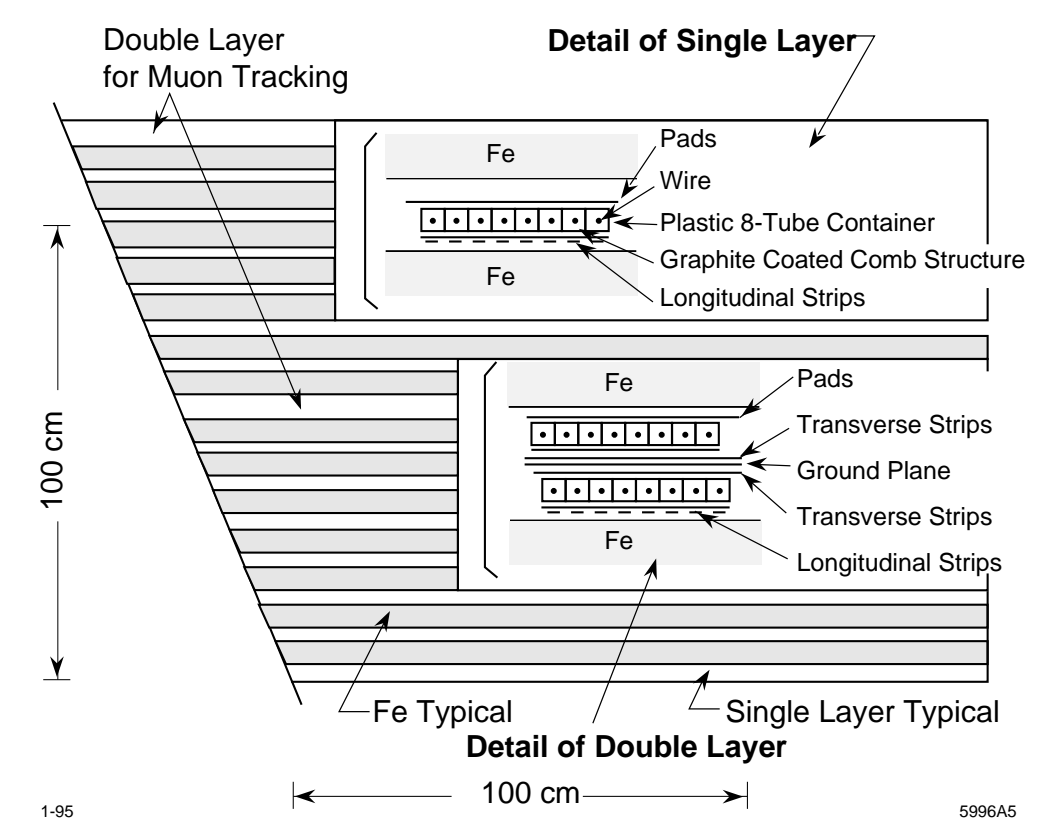

Figure 3.22: Placed on one side of each Iarroci tube are pads for calorimeter readout, and on the other side strips for muon identification. Transverse strips, placed in special double layers, supply coordinate information perpendicular to the Iarroci tubes for muon identification.

to provide a true or false hit record on every beam crossing.

The barrel chambers provide an overall efficiency of $85 \%$ for detecting penetrating muons (above about $2.5 \mathrm{GeV}$ ) out to a production angle of $|\cos \theta|<0.6$. Due to constraints imposed by strict earthquake standards at SLAC, there is something of a hole in the WIC coverage before the endcap module becomes useful between $0.74<|\cos \theta|<0.95$. For this reason, muon identification in the interim region must rely upon LAC shower information only, with a corresponding loss in efficiency. 


\subsection{Data Acquisition}

\subsection{Data Acquisition}

Monitoring and control of the SLD data acquisition, as well as other detector functions, is performed 'on-line' by various independent processes running on the SLD Vax cluster. The real work of the SLD data acquisition, however, is performed 'below-line' by an assortment of FASTBUS based processing modules which typically provide both event buffering and processing by means of embedded Motorola 68020 CPUs [63]. While the details of the data acquisition system vary somewhat for each SLD subsystem, in general the analog signals are conditioned and digitized by hybrid front end electronics modules mounted directly on the detector, and this data is then shipped serially over optical fiber links to the FASTBUS based processing modules. These FASTBUS modules apply various calibrations to the raw data, and then perform a wide variety of basic analysis tasks including waveform hit finding, rudimentary particle tracking, and the calculation of other useful quantities to provide information for the trigger decision. A trigger decision is made on every SLC beam crossing, and for all triggered interactions the data is collected from the various subsystems, packaged into an event, and written to a shared event pool on the SLDACQ Vax. At this stage, the various on-line processes can access this information to provide monitoring information and graphical one-event displays, and 


\section{The SLD Detector}

from this pool the events are eventually written to tape.

The FASTBUS modules for the various subsystems generate and store their own calibration constants by injecting reference charges into the front end electronics modules to generate an observed channel response curve. This calibration procedure cannot be performed while the detector is taking data, but rather the detector subsystems are recalibrated on a roughly daily basis when the opportunity presents itself.

Since the beginning of the 1994-95 SLD run the below-line data acquisition system has been fully pipelined, allowing the various subsystems to acquire data in an autonomous fashion so that the faster subsystems and the trigger do not incur any appreciable dead-time from the slower wire chamber subsystems which can take many beam crossings to complete the processing of a triggered event.

\subsection{Detector Simulation}

Due to the complexity of modern particle physics detectors, it has become common practice to generate simulated data, generally referred to as Monte Carlo, to aid in the understanding of a variety of instrumental and systematic effects. The process of generating Monte Carlo data is typically divided into three distinct phases. 


\subsection{Detector Simulation}

The first step, known as the generation phase, involves simulating some real physical process by generating momentum four vectors representing the produced particles from a set of theoretical and empirical cross sections. A number of packages are available to simulate the most common physics processes at the $\mathrm{Z}$ pole. The one used in this analysis is the Jetset 7.4 generator which simulates both the production and decay of hard hadron-hadron, hadron-lepton and lepton-lepton interactions [64].

The second step, known as the simulation phase, involves tracking these produced particles through a model of the SLD detector and simulating the interactions between these particles and the various detector components. Most of the work of the simulation phase is performed by the widely used GEANT detector simulation package [65]. For any simulated particle traversing an instrumented region of the detector, simulated raw data is generated at the individual hit level to match as closely as possible what would be observed in the real detector.

In the third step, known as the reconstruction phase, the simulated detector hits are overlayed with real detector noise sampled from a set of random detector triggers to produce realistic real life events. One random trigger is taken every 20 seconds during normal SLD data logging and provides a snapshot of the typical beam-related background present in the detector. These simulated events are then run through the full SLD reconstruction package so that a direct comparison can be 


\section{The SLD Detector}

made between the reconstructed or 'observed' quantities and the generated or 'true' values produced by the event generator.

In searches for rare decays, very few real data events, if any at all, will be seen and self calibrating techniques, where data itself is used for efficiency and purity studies, cannot be implemented. For this analysis, the SLD Monte Carlo provides a crucial and accurate tool for understanding the sensitivity and the expected background for the rare decay signals. Efforts are made to constrain MC properties with actual data wherever appropriate and possible as described in Chapter 4. 


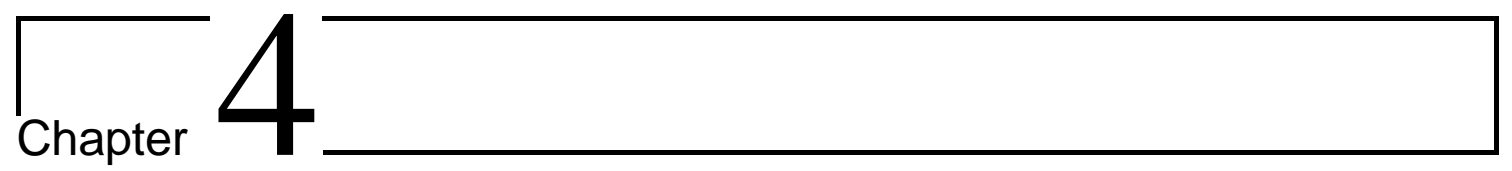

\section{Candidate Vertices and MC Calibration}

The SLD is a general purpose particle detector designed to efficiently detect all interesting physics processes generated by $e^{+} e^{-}$collisions near the $Z$ pole energy. For the most part, the triggering and reconstruction of the events observed by the SLD is shared among the various physics analyses performed on this data. From this common pool of interesting 'physics' events, an event selection is then performed by each particular analysis to isolate only that set of data which is useful in each particular instance. This chapter will briefly summarize the general detector triggering and reconstruction process, as well as describe the particular selection used in this analysis to identify and classify a preliminary event selection (candidate vertices) in 


\section{Candidate Vertices and MC Calibration}

rare hadronic $B$ decays. The last section will dwell on a set of corrections that have been applied on MC generated events.

\subsection{Detector Trigger}

The expected rate of interesting physics events produced by the SLC is on the order of two per minute. With a collision rate of $120 \mathrm{~Hz}$, it is infeasible to record every beam crossing seen by the SLD, and some amount of detector triggering logic is employed to reduce the amount of data written to tape. Compared to other particle physics experiments, the demands on the SLD trigger logic is quite mild, and a straight forward set of criteria are sufficient to reduce the detector trigger rate to an acceptable $0.3-2 \mathrm{~Hz}$. A variety of different triggers are independently evaluated to ensure the efficient detection of interesting physics events. As the trigger decision is made on every beam crossing, there is only a rudimentary amount of information available on which to base the trigger algorithm.

- The energy trigger requires minimum $8 \mathrm{GeV}$ of total deposited energy to be recorded by the electromagnetic (EM) and/or hadronic (HAD) calorimeter towers in the LAC. To eliminate noise, only towers above a certain threshold are counted in this energy sum. In the EM section this threshold is $60 \mathrm{ADC}$ 
counts $(246 \mathrm{MeV})$ and in the HAD section the threshold is 120 ADC counts (1296 MeV). To greatly reduce beam induced muons the energy trigger is vetoed if there are more than 1000 towers above a low threshold in the EM section. The low threshold is $8 \mathrm{ADC}$ counts in the EM section and $12 \mathrm{ADC}$ counts in the HAD section. Since October 1994 the entire detector is read out if the energy trigger is flagged. Prior to this only the LAC, WIC and LUM were read out.

- The tracking trigger requires minimum two charged tracks separated by at least $120^{\circ}$ in the CDC. At the trigger level, a set of hits is defined as a track if nine out of the possible ten CDC layers contain hits on a least six of the possible eight sense wires. This sequence of hits must also match a lookup table stored in the memory of the below-line FASTBUS modules. The table is comprised of all possible trajectories originating at the SLD IP with transverse momentum $>250 \mathrm{MeV}$. The track trigger will be vetoed if more than 275 of the 640 CDC cells satisfy the six hit criterion.

- The hadron trigger is a hybrid of the energy and track triggers, and requires at least one track plus over $2 \mathrm{GeV}$ of LAC energy. 


\section{Candidate Vertices and MC Calibration}

- The wide angle Bhabha trigger is designed to flag two back-to-back charged tracks in the CDC. Unlike the tracking trigger, this trigger allows for short track stubs, aimed at flagging wide angle Bhabha events $\left(e^{+} e^{-}\right.$events) to the maximum of the detector acceptance.

- The muon trigger requires one charged track to be present with 9 CDC layer hits. In addition, the opposite WIC octant must have calorimetric hits.

- The Bhabha trigger is aimed for the small angle Bhabha events seen in the luminosity monitor and only the LUM and the MASiC detectors are read out. It requires a total energy of minimum $12.5 \mathrm{GeV}$ (EM scale) in both the north and the south outer EM section of the luminosity monitor. The tower threshold is $1.25 \mathrm{GeV}$.

- The random trigger runs independently of the detector status and reads out the entire detector every 20 seconds for background overlays to be used in the MC generated event sample.

Unless otherwise stated the entire SLD detector was read out at the activation of either of the above triggers. Every triggered event observed by the SLD is written to tape for future processing. It has been estimated that the combination of the triggers listed above are intrinsically $100 \%$ efficient for triggering hadronic events 
[66]. However, due to the readout time of the CDC $(80 \mathrm{~ms})$, roughly $0.5 \%$ of the physics events will not be seen by the CDC. This together with the $4 \%$ down time of the CDC (voltage off) gives a maximum CDC efficiency of 95-96\%.

The rate of triggered data which is written to tape is still a factor of at least 30 larger than the expected interesting physics rate. Due to the approximately 2 seconds CPU time to fully reconstruct an event (mainly due to the tracking reconstruction) a filter stage is implemented where further requirements are imposed to reduce the event sample before a full reconstruction takes place. The energy filter is build on the energy trigger philosophy. The energy sum described under the trigger description will be named Ehi. Another energy sum which is the sum of all towers above a low threshold will be named Elo, where the low thresholds are 8 ADC counts in the EM section and $12 \mathrm{ADC}$ counts in the HAD section. The energy filter requires that the high energy sum Ehi must be larger than $15 \mathrm{GeV}$ (was $8 \mathrm{GeV}$ for the trigger), the low energy sum must be less than $140 \mathrm{GeV}$, the relationship Elo $<\frac{2}{3} \mathrm{Ehi}+70 \mathrm{GeV}$ must be satisfied and the number of towers above the low threshold must be larger than 9 . This greatly reduces the low energy and the high energy backgrounds while keeping essentially all physics events. To enhance the tagging efficiency of physics events that comes together with large backgrounds (events that would not pass the Elo < $140 \mathrm{GeV}$ and Elo $<\frac{2}{3} \mathrm{Ehi}+70 \mathrm{GeV}$ requirements) a separate tau filter is added. The 


\section{Candidate Vertices and MC Calibration}

tau filter is satisfied if the scalar momentum sum from all observed charged tracks is larger than $1 \mathrm{GeV}^{1}$. Essentially all the hadronic physics events passes either of these two filters, while the total event sample is reduced to around twice the physics sample. A full reconstruction is now applied to the remaining events by the off line reconstruction package.

The process of reconstruction involves turning the observed raw detector hits into physically motivated objects like tracks representing the passage of charged particles. Each detector component is first reconstructed individually: tracks are found in the CDC, energy clusters are formed in the LAC, Čerenkov rings are found in the CRID, etc. After all of these fundamental objects are found, the data from the various detectors which appear to be related to the same physical particle are connected, or linked, across all of the detector components into one logically grouped data structure.

The analysis herein only uses tracking information, and the algorithm for reconstructing tracks in the CDC is given in subsection 3.3.1. These tracks are then matched with hits in the vertex detector by extrapolation of the tracks from the CDC. If matching hits are found in the vertex detector this added information will

\footnotetext{
${ }^{1}$ At the filter stage, a somewhat more sophisticated fast tracking algorithm is applied compared to the one at the trigger level, although still far short of the full tracking reconstruction that will eventually be performed.
} 
113

\subsection{Candidate Vertices, a A Second Stage Filter}

be included in the overall track parameter fit.

\subsection{Candidate Vertices, a A Second Stage Filter}

Up to here the reconstruction is generic to all analyses and the basic physics information of interest is now available to select and classify the rare $B$ decays. This includes track information of the momentum, position and the charge. Everything else of interest for this analysis will then be reconstructed. In order to further reduce the event sample a second stage filter is implemented specifically for rare hadronic $B$ decays and events passing this filter will be named candidate vertices as explained in this section. The main analysis is performed on candidate vertices and is described in Chapter 5. The construction of the candidate vertices and the main analysis is fully based upon MC generated events.

The basic algorithm is to loop over all two track, three track and four track combinations for $B \rightarrow P P, B \rightarrow P V$ and $B \rightarrow V V$ modes respectively, where the total charge of the combinations must be 0 for the two and four track combinations and \pm 1 for the three track combinations. To reduce the possible combinations, each hadronic event is divided into two hemispheres as defined by the thrust axis [68]. Figure 4.1 shows a two jet event caused by the production of a quark anti-quark 


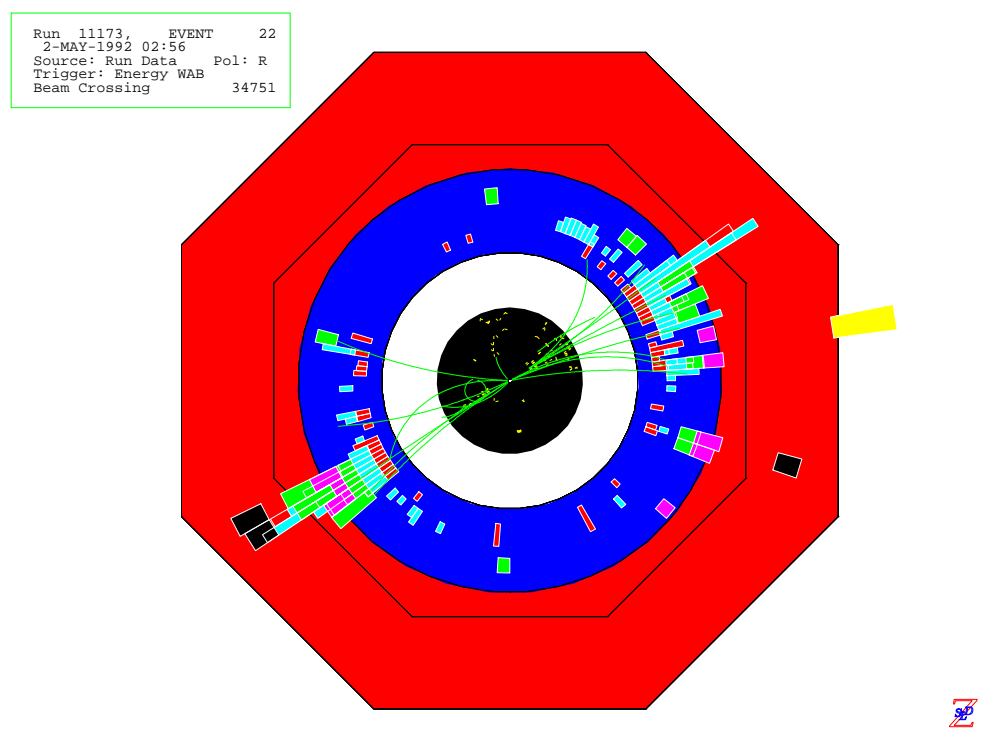

Figure 4.1: A typical $e^{+} e^{-} \rightarrow$ quark anti-quark event. Each event is divided into two hemispheres, separating the jets from each other. The candidate vertices are formed from track combinations that belong to the same hemisphere.

pair. Each jet will then lie in a separate hemisphere and only tracks from the same jet will be considered when the candidate vertices are constructed. To ensure that only well defined tracks are included a few requirements are imposed:

- Each track must have a momentum larger than $200 \mathrm{MeV}$.

- Each track must have at least 25 hits in the CDC.

- Each track must be matched to at least one hit in the vertex detector.

- The track must be within $5 \mathrm{~cm}$ of the SLD IP in the beam line direction when extrapolated to the point of closest approach to the SLD IP (POCA). 


\subsection{Candidate Vertices, a A Second Stage Filter}

- The chisquare of the fitted track in the CDC is required to be less than 15 per degree of freedom (number of CDC hits). For the combined vertex and CDC fit the chisquare must also be less than 15 for each degree of freedom. Here the CDC parameters counts as 5 degrees of freedom and each vertex hit counts as two degrees of freedom².

On average there are around 10 charged tracks in each hemisphere and the number of possible combinations that satisfy the charge requirement mentioned above are fairly high. If we assume that these 10 tracks are equally distributed between negative and positive charge there are 25 possible two track combinations, 100 possible three track combinations and 200 possible four track combinations for each hemisphere.

The next preselection requirements follow from a consideration based on the $B$ meson fragmentation function, which is the probability that a hadron will be created with a certain fractional energy of a quark. For hadrons consisting of a charm quark or a bottom quark the fragmentation function that very well models the measured data is the Peterson function [67], which is shown in Figure 4.2. A parameter $\varepsilon$ is set to 0.06 for charm hadrons and 0.006 for bottom hadrons. These parameters have been measured in semileptonic and partial reconstructed $B$ decays at SLD and LEP.

\footnotetext{
${ }^{2} \mathrm{~A}$ reconstructed CDC track at the point of closest approach to the SLD IP is parameterized by its curvature, azimuthal angle, polar angle, radial position and axial position. Each vertex hit is parameterized by azimuthal and polar angle coordinates.
} 


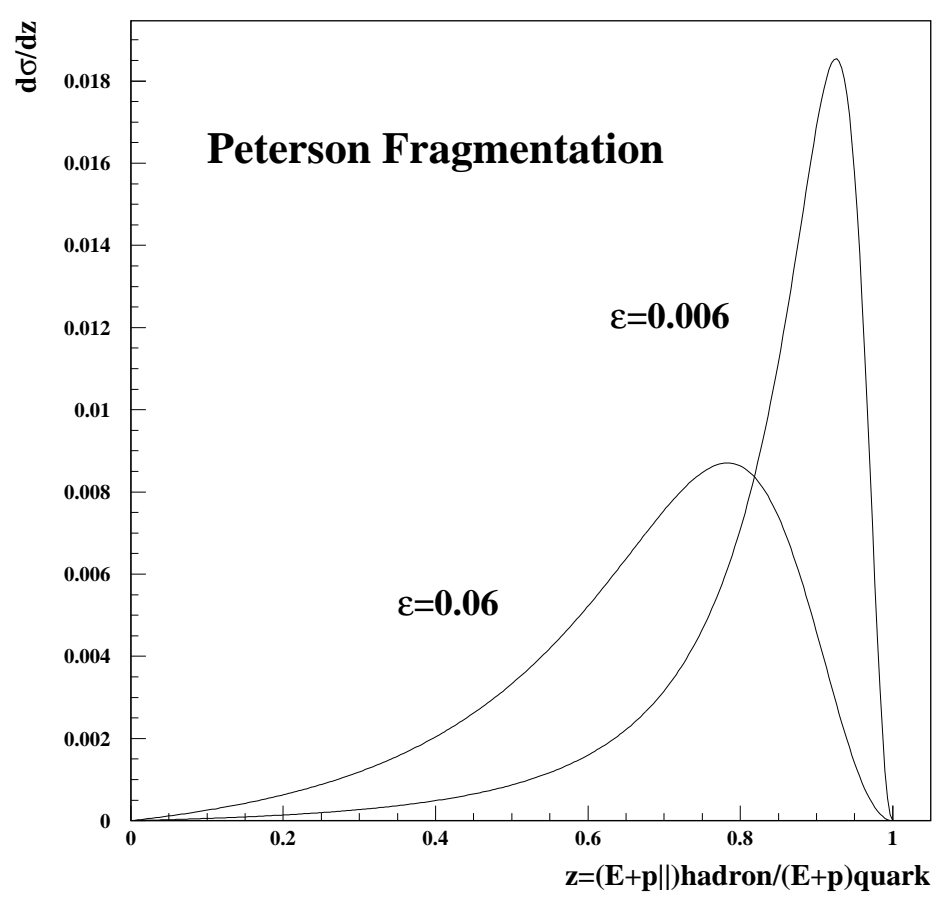

Figure 4.2: Peterson fragmentation function is shown for $c$ hadrons $(\varepsilon=0.06)$ and $b$ hadrons $(\varepsilon=0.006)$.

Translated into the corresponding mesons average fraction of the beam energy the mean values are 0.5 for charm hadrons and 0.7 for bottom hadrons. This tells us that the $B$ meson will be heavily boosted with an average energy of around $30 \mathrm{GeV}$ $(0.7 \times$ beam energy $)$. Thus, any decay products of a $B$ will be boosted forward in the $B$ flight direction with a small spread between the decay products. Figure 4.3 shows the fragmentation parameter and the maximum angle between two tracks for a set of fully reconstructed $B$ meson MC events. The left plot in Figure 4.4 shows 


\subsection{Candidate Vertices, a A Second Stage Filter}
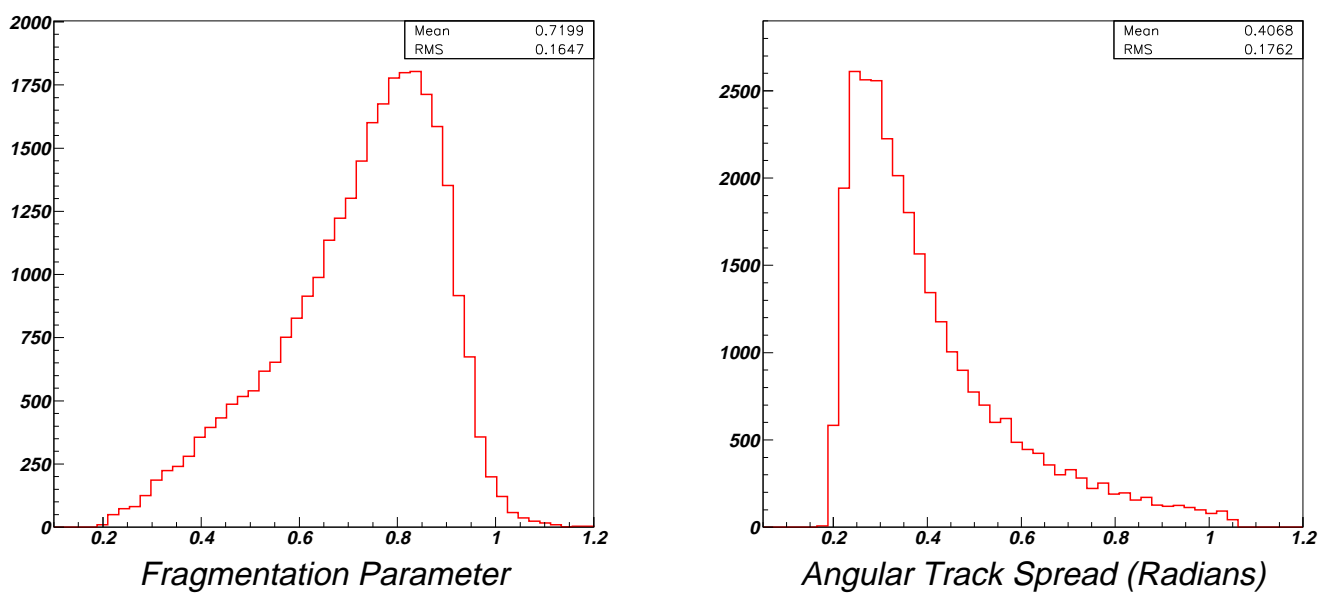

Figure 4.3: The fragmentation parameter and the largest angular spread between any tracks in a fully reconstructed $B$ meson MC event is shown.

the momentum distribution of the stiffest track.

The first preselection cuts are then

- a cut on the maximum angle between any tracks that combines to form a candidate vertex. The cut is set at $\pi / 3$ radians,

- the candidate vertex must at least have $20 \%$ of the beam energy,

- the momentum of the stiffest track must exceed $4 \mathrm{GeV}$.

The next set of preselection requirements is based on the reconstructed $B$ meson mass and the vector mesons, where the $B$ meson mass is calculated from the individual tracks $i$ that form a vertex candidate, 


\section{Candidate Vertices and MC Calibration}

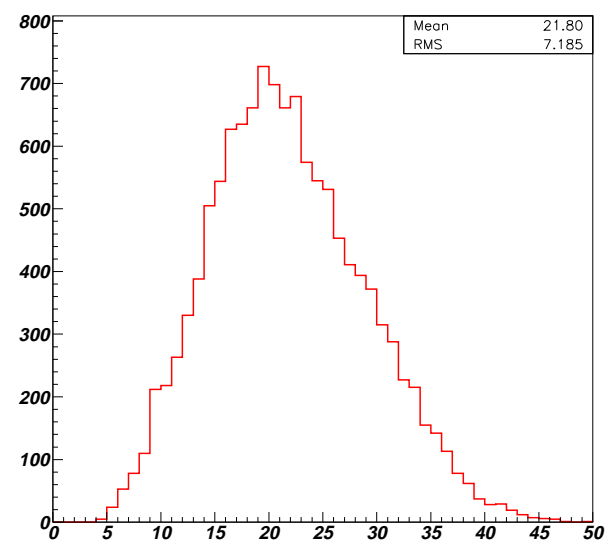

Stiffest Track Momentum (GeV)

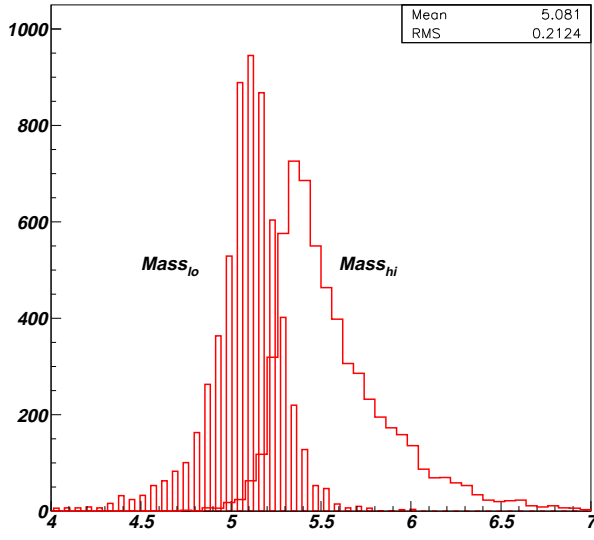

Reconstructed Vertex Mass (GeV)

Figure 4.4: The momentum distribution for the stiffest track and the vertex mass distribution in a fully reconstructed $B$ meson MC event is shown for $B^{+} \rightarrow$ $K^{* 0} K^{+}$.

$$
M_{B}^{2}=\left(\sum_{i} \sqrt{m_{i}^{2}+P_{i}^{2}}\right)^{2}-\left(\sum_{i} P_{x i}\right)^{2}-\left(\sum_{i} P_{y i}\right)^{2}-\left(\sum_{i} P_{z i}\right)^{2}
$$

where $m_{i}$ is the mass of track $i, P_{i}$ is the total momentum of each track and $P_{x i}$, for example, is the $\mathrm{x}$ momentum component of track $i$. For the various modes there is a specific combination of pions and/or kaons in the final states, but at this stage no assignment has been attempted on a track by track basis. Two vertex masses are instead formed, one where all tracks are assigned a pion mass masslo and one where all tracks are assigned a kaon mass mass h . The right plot in Figure 4.4 shows the reconstructed vertex masses $\left(\right.$ mass $_{l o}$ and mass $\left._{h i}\right)$ for $B^{+} \rightarrow K^{* 0} K^{+}$.

- It is required that mass $s_{l o}$ is less than $6.4 \mathrm{GeV}$ and that mass $s_{h i}$ is larger than 


\subsection{Candidate Vertices, a A Second Stage Filter}

4.7 GeV. For the three and four track vertices a requirement of mass $l_{0}>4.0$ $\mathrm{GeV}$ is also included. With three or four tracks the difference between masslo and mass $s_{h i}$ can become quite substantial for background events and justifies a lower cut on both mass $l_{l o}$ and mass $s_{h i}$. This difference is less apparent for two track modes and the lower cut on mass lo $_{0}$ is not applied since the lower cut on mass $s_{h i}$ only is sufficient to reduce large portions of background events.

Most of the modes contain a vector meson, and two of the tracks (with total charge zero) in a candidate vertex must combine to give the vector mass. For $B^{+} \rightarrow P^{+} V^{0}$ it is possible by a straightforward calculation to show that out of the two possible two track combinations, it is always the combination with the smallest mass that is the right one $\mathrm{e}^{3}$. This combination is then used to calculate the vector mass, first when both tracks are assigned pion masses $m_{l o}$ and secondly when the tracks are assigned kaon masses $m_{h i}$. A preselection cut is then made for the three track vertices,

- $m_{l o}$ must be less than $1.5 \mathrm{GeV}$ and $m_{h i}$ must be larger than $0.4 \mathrm{GeV}$.

This is well within the vector mass range $\left(m_{\rho}=0.7 \mathrm{GeV}, m_{K^{*}}=0.9 \mathrm{GeV}\right.$ and $\left.m_{\phi}=1.0 \mathrm{GeV}\right)$. For the four track vertices the possible combinations are somewhat

\footnotetext{
${ }^{3}$ This result is valid when light vector mesons are in consideration. For other modes with a heavier vector, for example $B^{+} \rightarrow D^{0} \pi^{+} \rightarrow K^{-} \pi^{+} \pi^{+}$, it is not necessarily correct.
} 


\section{Candidate Vertices and MC Calibration}
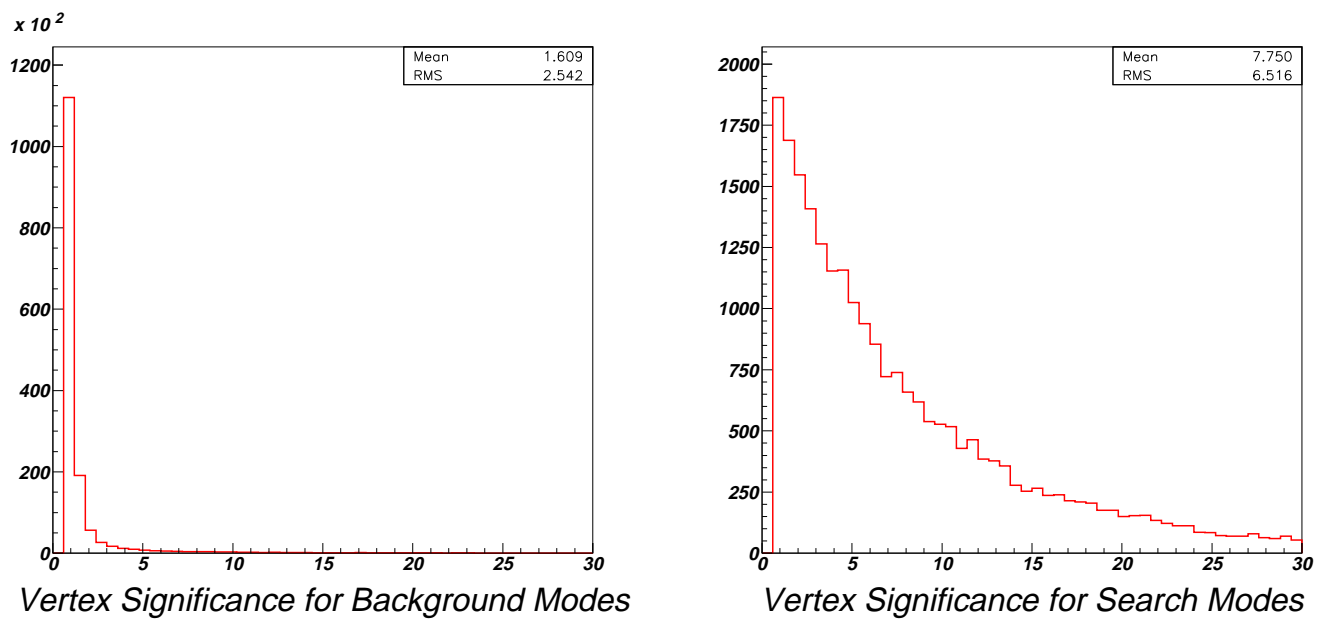

Figure 4.5: Vertex significance for background and search modes are shown after the cut at 0.6. Most of the background events are expected to originate at the SLD IP, and a cut at 0.6 greatly reduces the background sample.

more complex and a corresponding cut on the vector masses is not applied at this stage.

The cuts mentioned so far reduce the sample of the search modes to a very small extent, while greatly reducing background events. The last two cuts in this preliminary event selection, which follows, are of a slightly different nature in that they reduce the signal size somewhat. The 'vertex significance' is the $B$ vertex normalized flight distance from the SLD IP (Decay Length/Uncertainty). With a typical $B$ decay length of $2 \mathrm{~mm}$ and an uncertainty in the SLD IP position of only a few microns it is expected that this parameter will separate out events that originate directly 


\subsection{Candidate Vertices, a A Second Stage Filter}

at the SLD IP as opposed to $B$ mesons, in which the $B$ is expected to travel some distance before decaying $(2 \mathrm{~mm})$. The added uncertainty from the reconstructed $B$ vertex position will degrade this separation somewhat, but still, at SLD an excellent vertex significance resolution is achieved due to the high resolution vertex detector and small beam spots as explained in Chapter 3 . The vertex significance is shown for non-search modes (background) and the search modes in Figure 4.5 and a cut is made which reduces the number of background events by a many orders of magnitude,

- the vertex significance parameter must be larger than 0.6.

A last cut is made on the vertex probability,

- the probability that the candidate tracks come from the same vertex must be larger than $1 \times 10^{-5}$.

Although this is a somewhat loose cut it still discards a large number of background vertices for which the candidate tracks do not actually come from a common origin. Vertices that pass the above second stage filter are named candidate vertices and are written to separate tapes for further analysis. Table 4.1 lists the number of $\mathrm{MC}$ generated events for the search modes and the fraction that remains after the implementation of this stage in the analysis. The events that are lost for the 


\section{Candidate Vertices and MC Calibration}

\begin{tabular}{|l|r|r||l|r|c|}
\hline \hline Type & $\begin{array}{r}\text { Events } \\
\text { Generated }\end{array}$ & $\begin{array}{c}\text { Fraction } \\
\text { Left }\end{array}$ & Type & $\begin{array}{r}\text { Events } \\
\text { Generated }\end{array}$ & $\begin{array}{c}\text { Fraction } \\
\text { Left }\end{array}$ \\
\hline$B \rightarrow P P$ & & & $B^{0} \rightarrow V V$ & & \\
\hline$B^{0} \rightarrow \pi^{+} \pi^{-}$ & 9355 & 0.53 & $B^{0} \rightarrow \rho^{0} \rho^{0}$ & 21251 & 0.39 \\
$B^{0} \rightarrow K^{-} \pi^{+}$ & 9298 & 0.53 & $B^{0} \rightarrow \bar{K}^{* 0} \rho^{0}$ & 14023 & 0.40 \\
$B^{0} \rightarrow K^{+} K^{-}$ & 28495 & 0.56 & $B^{0} \rightarrow \bar{K}^{* 0} K^{* 0}$ & 3523 & 0.41 \\
$B_{s} \rightarrow \pi^{+} \pi^{-}$ & 3224 & 0.56 & $B^{0} \rightarrow \phi \rho^{0}$ & 3936 & 0.36 \\
$B_{s} \rightarrow K^{+} \pi^{-}$ & 5218 & 0.56 & $B^{0} \rightarrow \phi \bar{K}^{* 0}$ & 2654 & 0.38 \\
$B_{s} \rightarrow K^{+} K^{-}$ & 5187 & 0.53 & $B^{0} \rightarrow \phi \phi$ & 1945 & 0.34 \\
\hline$B \rightarrow P V$ & & & $B_{s} \rightarrow V V$ & & \\
\hline$B^{+} \rightarrow \rho^{0} \pi^{+}$ & 25439 & 0.44 & $B_{s} \rightarrow \rho^{0} \rho^{0}$ & 1868 & 0.38 \\
$B^{+} \rightarrow \rho^{0} K^{+}$ & 13718 & 0.46 & $B_{s} \rightarrow \bar{K}^{* 0} \rho^{0}$ & 1734 & 0.41 \\
$B^{+} \rightarrow K^{* 0} \pi^{+}$ & 17150 & 0.45 & $B_{s} \rightarrow \bar{K}^{* 0} K^{* 0}$ & 1744 & 0.38 \\
$B^{+} \rightarrow K^{* 0} K^{+}$ & 17226 & 0.44 & $B_{s} \rightarrow \phi \rho^{0}$ & 1502 & 0.38 \\
$B^{+} \rightarrow \phi \pi^{+}$ & 8763 & 0.40 & $B_{s} \rightarrow \phi K^{* 0}$ & 1375 & 0.36 \\
$B^{+} \rightarrow \phi K^{+}$ & 8727 & 0.41 & $B_{s} \rightarrow \phi \phi$ & 1428 & 0.36 \\
\hline
\end{tabular}

Table 4.1: The modes that are searched for and the corresponding amount of MC generated events. Also shown are the fraction of the search modes that remains after the second stage filtering is implemented. The efficiencies quoted are for the charged final states only and for modes involving a vector meson that also decays into neutral tracks, the overall efficiency will be reduced accordingly (subsection 1.3.1).

various modes are primarily due to decays of the $B$ outside the fiducial volume of the detector. When a $B$ is produced towards the endcap region (large $|\cos \theta|$ with $\theta$ being the polar angle) the probability for reconstructing the final tracks becomes small. This effect increases with the number of tracks in the final state. Only to a smaller degree do the above cuts lower the search mode sample. From a sample of 2.5 million multi hadronic MC generated background events (approximately four times the data sample), there are around 7000,45000 and 150000 candidate vertices 


\subsection{Calibration of the MC}

for the two track modes, three track modes and four track modes respectively.

\subsection{Calibration of the MC}

MC generated events are at some level used by most of the analyses at SLD, and depending on the nature of the particular study, the MC becomes more or less important. In some analyses a large and well separated signal can be found in data and the calibration of the analyses can be done directly on the data itself. In the search for rare $B$ decays, however, very few events will be seen in the data, if any, and any form of self calibration techniques will be out of the question. Hence, the analysis relies heavily on the accuracy of the $\mathrm{MC}$ generated events and this section will address the performance of the MC.

At SLD the MC has a history of excellent performance, and discrepancies between data and MC are small. This is particularly true for the track reconstruction in the central drift chamber, causing this analysis to start at a level of high confidence. In section 3.3 a few plots were shown of the comparison between MC and data for several distributions relevant to CDC. Figures 3.13 and 3.14 shows that there are good agreement for hits in the CDC. 


\section{Candidate Vertices and MC Calibration}

\subsubsection{Tracking Efficiency}

Of great importance to this analysis is the CDC and VXD track reconstruction efficiency. If a single track reconstruction efficiency is $T$ the efficiency for reconstructing a vertex with $n$ tracks is $T^{n}$ (if correlations are ignored). Any discrepancy between MC and data will then be amplified with increasing number of tracks and a detailed understanding of the $\mathrm{MC}$ versus the data efficiencies are necessary.

At SLD there exists a set of standard routines that are routinely used by many groups in a wide range of analyses. The typical approach is to bin the track distribution versus polar angle and track momentum and throw away a small fraction of the reconstructed MC tracks in order to match the tracking efficiencies seen in the data bin by bin. The standard routines deal mainly with tracks in the momentum region $0-2 \mathrm{GeV}$ and to a lesser degree addresses the tracking efficiency issue for tracks with large momentum. The analysis herein deals essentially with hadronic events with largely boosted tracks and it turns out that the analysis suffers unfairly when the standard routines are applied. Even for a four-body decay the mean track momentum is almost $10 \mathrm{GeV}$. Thus, a separate study was performed for the tracking efficiency with an approach more tailored to high momentum tracks, as follows.

In a $B$ event at the SLD there are two types of tracks, the tracks associated 


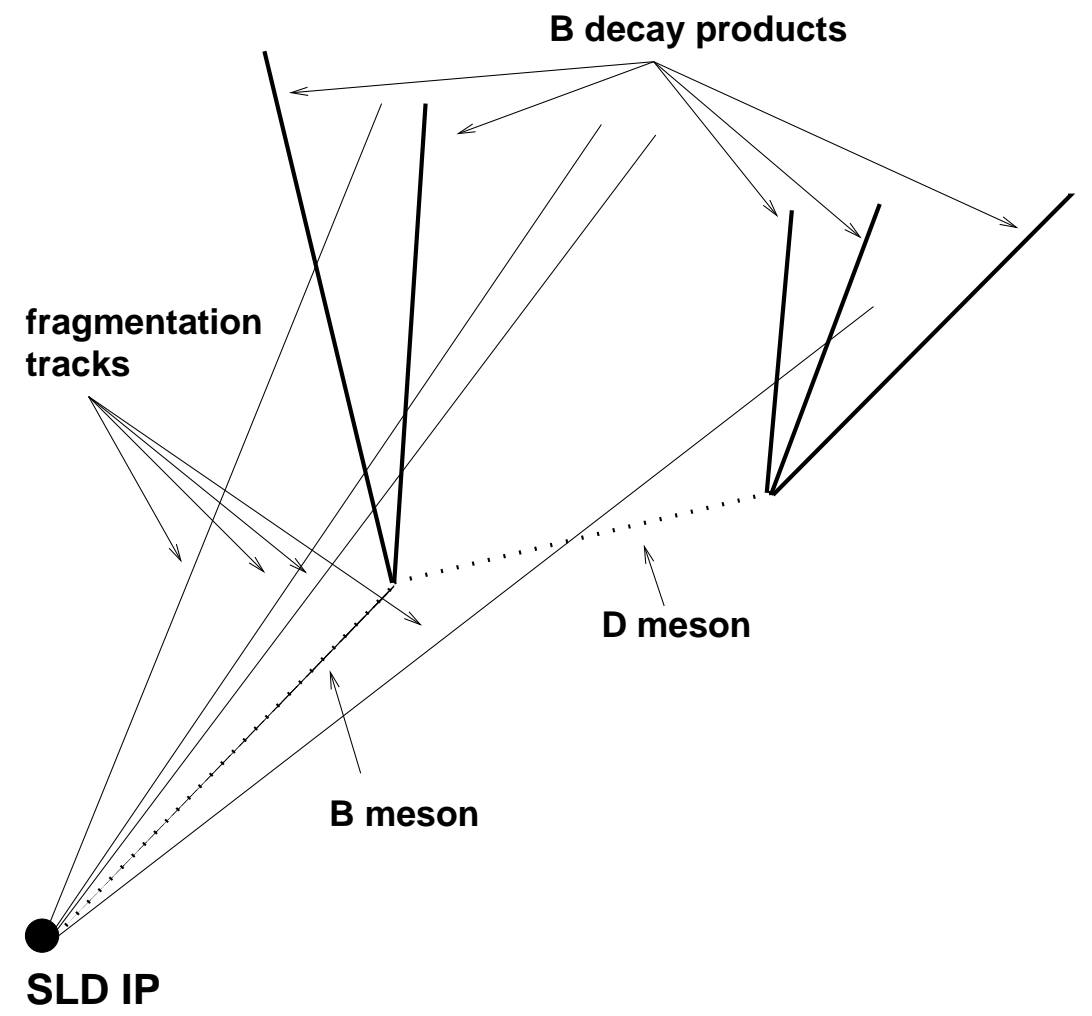

Figure 4.6: A track is classified as a fragmentation track or a track from the $B$ decay.

with the $B$ event, and the generally prompt fragmentation tracks which are a result of the general hadronization process and are not associated with a $B$ decay. This classification is shown in Figure 4.6 for a $B \rightarrow D X$ event. The thin lines illustrate fragmentation tracks while the thicker lines are associated with the $B$ meson decay. We split this study into the VXD2 and the VXD3 periods due to the different performance of the track reconstruction for these periods. 


\section{Candidate Vertices and MC Calibration}

\begin{tabular}{||l|c|c|l||} 
Track type & VXD2 & VXD3 & Info from \\
\hline From B & 0.97 & 0.95 & CLEO \\
\hline Overall & 1.02 & 1.00 & SLD,OPAL,DELPHI \\
\hline Fragmentation & 1.06 & 1.05 & Deduced \\
\hline
\end{tabular}

Table 4.2: Weight Constants Applied to the MC Tracks

The idea behind the study is to use CLEO measurements [27] to constrain the number of tracks coming from the decay of the $B$ hadron, and $Z^{0}$ data on the total multiplicity of $b \bar{b}$ events $\left(\mathrm{SLD}^{4}\right.$ [28], DELPHI [29] and OPAL [30]) to constrain the number of fragmentation tracks in $b \bar{b}$ events. Constraining the $\mathrm{MC}$ at the generator level in this way provides a well understood comparison between tracking multiplicity in data and MC for identified $Z \rightarrow b \bar{b}$ events. Table 4.2 shows the corrections applied to the generator level MC in order to get a good agreement with the CLEO and the $Z^{0}$ data. It is found that the generated $B$ hadron decay multiplicity is $3 \%(5 \%)$ too high for the VXD2 (VXD3) MC, while the generated fragmentation multiplicity is $6 \%(5 \%)$ too low for the VXD2 (VXD3).

The next step in this study is to investigate the track multiplicity in the SLD reconstructed $b \bar{b}$ sample. This sample is also used in the $R_{b}$ analysis and is reconstructed from topological vertexing and a $2 \mathrm{GeV}$ mass tag, giving a $98 \%$ pure $b \bar{b}$

\footnotetext{
${ }^{4}$ Measured with added information of the total tracking multiplicity which is measured at LEP to $1 \%$ precision.
} 


\subsection{Calibration of the MC}
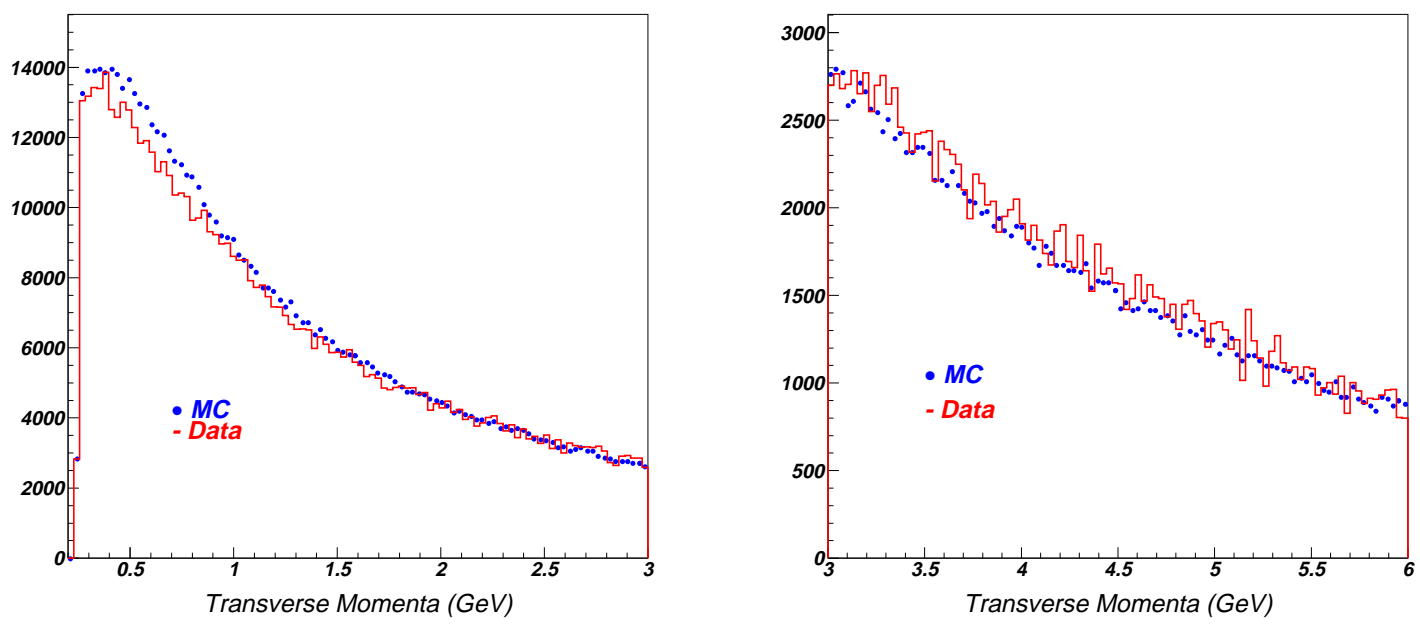

Figure 4.7: Shown are the track multiplicity in the $1996-97 R_{b} b \bar{b}$ sample. Data and MC are compared versus the track momentum.

sample [31]. Figures 4.7 and 4.8 compares distributions of tracks reconstructed in MC (with the above re-weights applied) and data for the $b \bar{b}$ events. The left plot shows a $6 \%$ excess of tracks in the VXD3 MC for tracks with $p_{t}$ less than $0.8 \mathrm{GeV}$, relatively evenly distributed over the $\cos \theta$ range. Above $p_{t}$ of $0.8 \mathrm{GeV}$ good agreement is seen between data and MC. Based on this study, and a similar study for the VXD2 sample, it was decided to prepare the MC by throwing away $6 \%$ of the MC tracks below $1.5 \mathrm{GeV}$ and $0.8 \mathrm{GeV}$ for the VXD2 and the VXD3 sample respectively. 


\section{Candidate Vertices and MC Calibration}
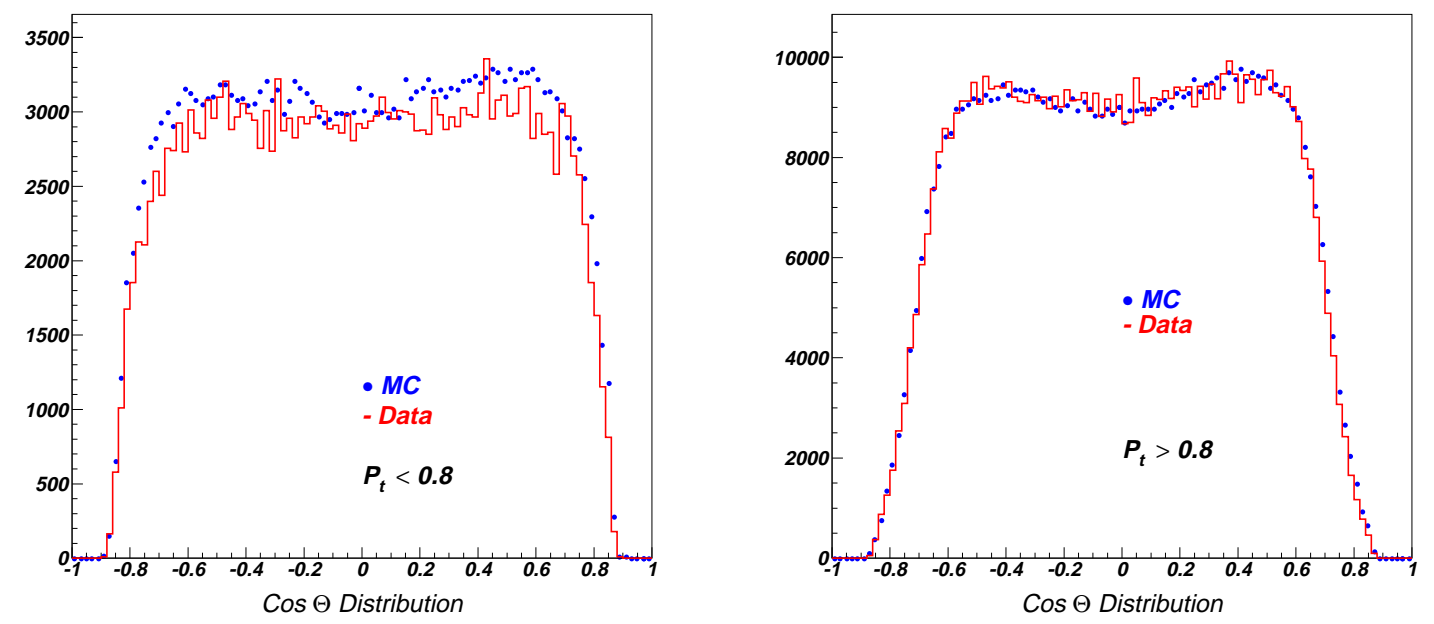

Figure 4.8: Shown are the track multiplicity in the 1996-97 $R_{b} b \bar{b}$ sample. Data and MC are compared versus the track polar angle $\cos \theta$ for two regions of transverse momentum.

\subsubsection{Momentum Smearing}

A issue of great importance is the understanding of the momentum resolution for reconstructed tracks. Any mismatch between data and $\mathrm{MC}$ would lead to differences in the reconstructed vertex mass and the vector masses to which this analysis is very sensitive. The $D^{+} \rightarrow K^{-} \pi^{+} \pi^{+}$event sample provides a well understood system for comparison between data and MC. The standard SLD $D^{+} \rightarrow K^{-} \pi^{+} \pi^{+}$event selection is described in [28]. The idea is to look at the mass width of the reconstructed $D$ mesons, and to use this as a measure for possible discrepancies in momentum 


\subsection{Calibration of the MC}
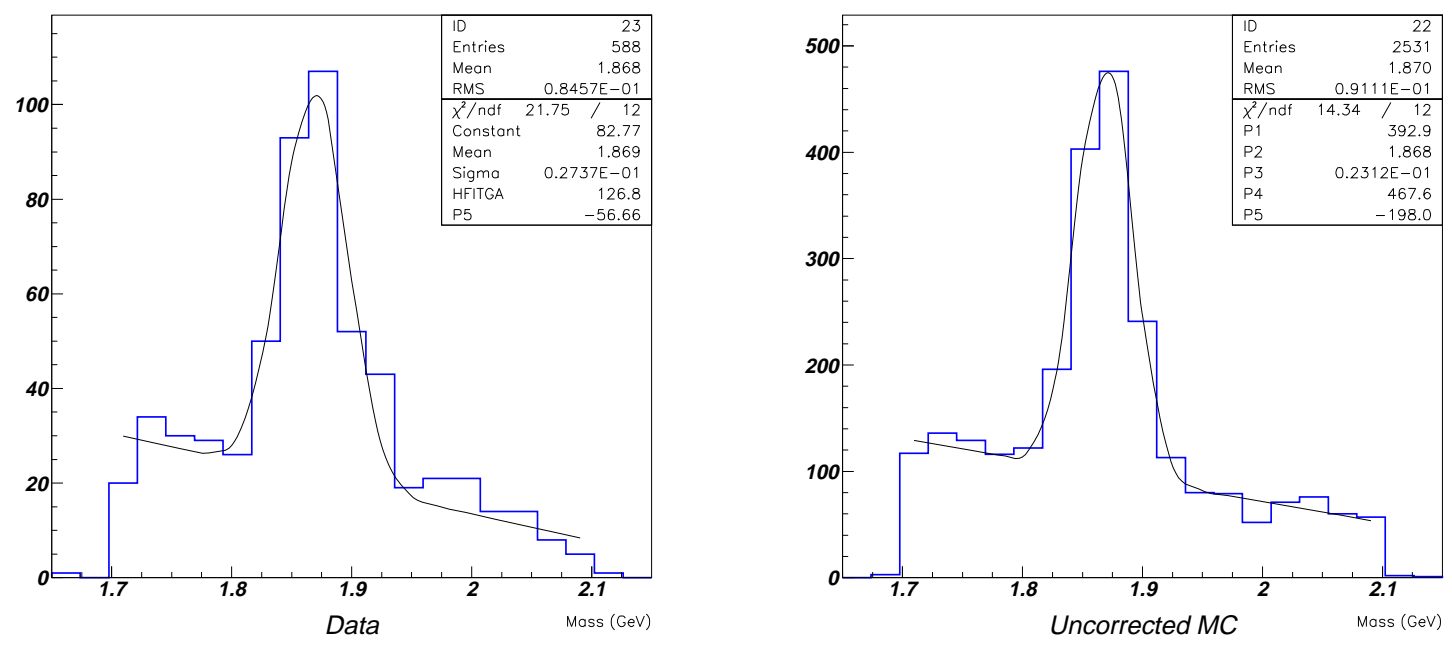

Figure 4.9: The $D^{+} \rightarrow K^{-} \pi^{+} \pi^{+}$mass width is shown for both data and uncorrected $\mathrm{MC}$.

resolution between data and MC. Figure 4.9 shows the reconstructed vertex mass $M_{d}$ for data and uncorrected MC (1996-97 event sample). The fit curve shown is a straight line (background) plus a Gaussian (signal),

$$
\text { Fit Curve }=P_{1} e^{-\frac{\left(M_{d}-P_{2}\right)^{2}}{2 P_{3}^{2}}}+P_{4}+P_{5} M_{d}
$$

and the parameters $P_{i}$ can be seen in the Figure. A discrepancy in the mass width of around $4 \mathrm{MeV}$ can be seen. The momentum resolution, which is determined by the single hit resolution and random alignment error, is Gaussian in $1 / p_{t}$ (radius of curvature). By adding additional smearing to the MC transverse momentum for each track, it is found that the mass distribution of the respective mode agrees well. 


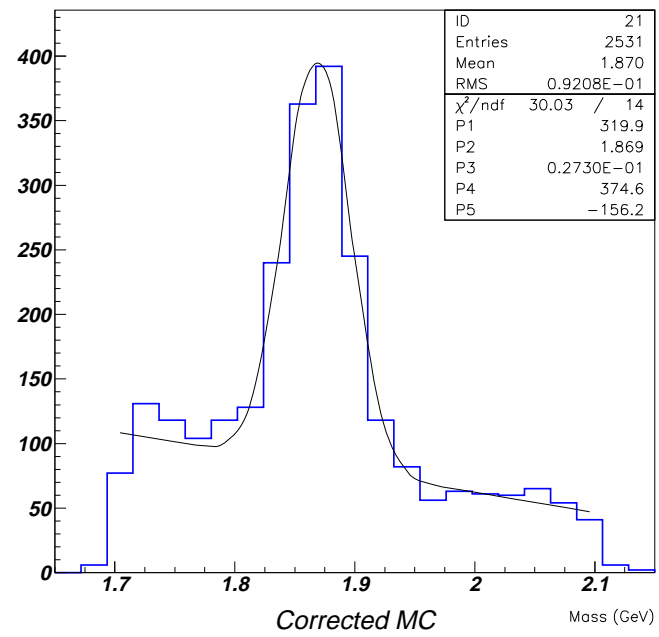

Figure 4.10: The $D^{+} \rightarrow K^{-} \pi^{+} \pi^{+}$mass width is shown for corrected MC.

The smearing of the transverse momentum $p_{t}$ follows the rule

$$
\frac{1}{p_{t}} \rightarrow \frac{1}{p_{t}}+\text { Gaussian }
$$

where Gaussian is a random Gaussian variable with width 0.001 . A similar study was done on VXD2 events, and the MC and the data agrees if a Gaussian variable with width 0.002 is used. Figure 4.10 shows the MC mass distribution for the 1996 97 sample after the correction. As a cross check of this result, the MC and data mass distributions was compared for the $K_{s}$ event sample. It was found that the $K_{s}$ sample is insensitive to this due to low track momentum, and that the MC and data agrees well. This smearing is consistent with similar results found by other 


\subsection{Calibration of the MC}
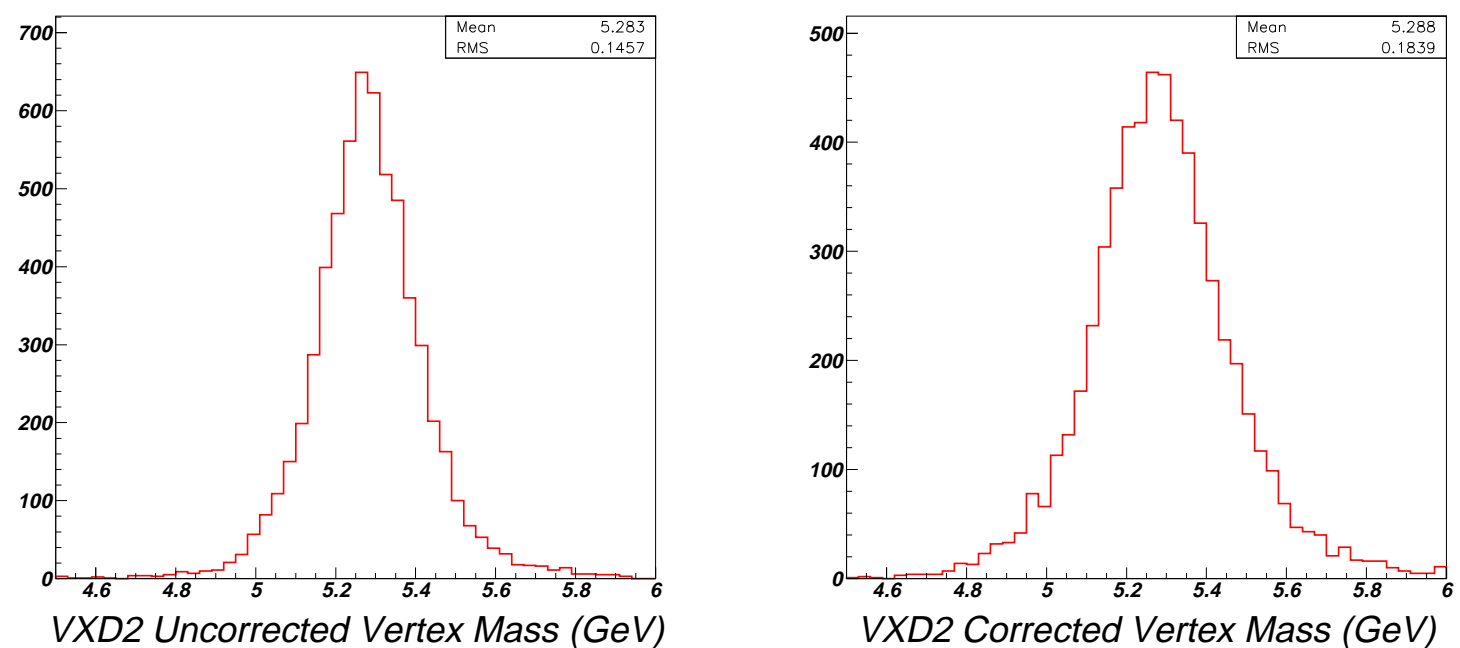

Figure 4.11: The reconstructed $B$ meson mass is shown for the $B^{+} \rightarrow \rho^{0} \pi^{+}$mode during the VXD2 run period. The left plot shows the reconstructed mass when no additional $p_{t}$ smearing is applied and in the right plot the mass is shown after $p_{t}$ smearing.

collaborators at SLD and ultimately increases the width of reconstructed $B$ meson masses in rare $B$ decays. For the VXD2 period this effect is largest and for the $B^{+} \rightarrow \rho^{0} \pi^{+}$mode an increase in the mass width from $146 \mathrm{MeV}$ to $184 \mathrm{MeV}$ is observed (Figure 4.11). A similar effect is seen for the vector meson masses.

\subsubsection{Vertexing Resolution}

By investigating the full hadronic event sample a comparison can be done on the impact parameters at the point of closest approach. Figure 4.12 shows a track 


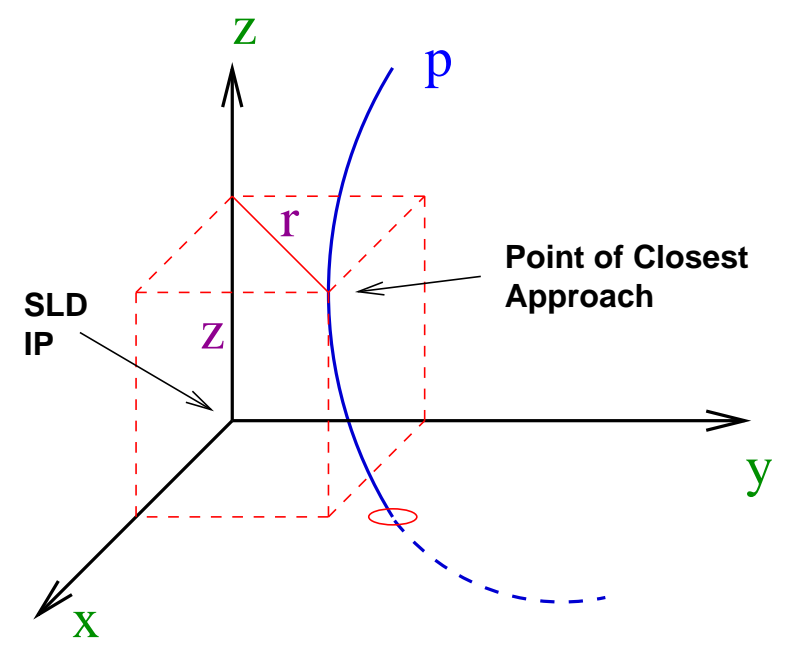

Figure 4.12: A track with cylindrical coordinates $r$ and $z$ at the point of closest approach.

with cylindrical coordinates $r$ and $z$ at the point of closest approach. It was found after a comparison between data and MC of the width of $r$ and $z$ distributions, that the resolution agrees if a convoluted set of smearing is applied on $r$ and $z$. Standard SLD routines are applied for this. Figure 4.13 shows the normalized track impact parameter, which will be discussed in the next chapter, before and after the vertexing resolution corrections for the $B^{+} \rightarrow \rho^{0} \pi^{+}$mode during the VXD3 run period. A three percent increase in this parameter can be seen resulting from the vertex resolution smearing. 
133

\subsection{Calibration of the MC}
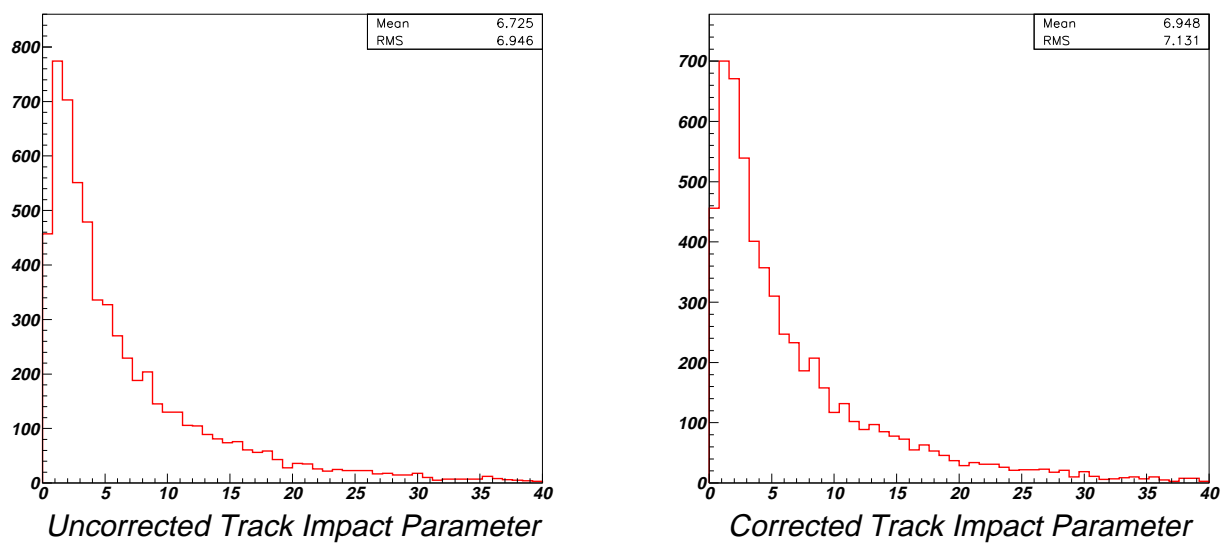

Figure 4.13: The reconstructed normalized track impact parameter is shown for the $B^{+} \rightarrow \rho^{0} \pi^{+}$mode during the VXD3 run period. The left plot shows this parameter when no additional vertex resolution smearing is applied and in the right plot the impact parameter is shown after the smearing. 



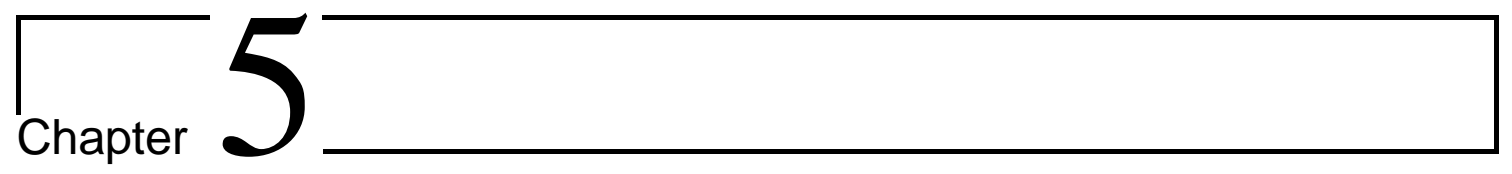

\section{The Rare Decay Event Selection}

\section{Algorithm}

This chapter will describe the main event selection for tagging rare $B$ decays. The starting point is the candidate vertices as defined in Chapter 4, where the candidate vertices satisfy a minimum set of requirements in the second stage filter. The simple filter allowed for a considerable reduction of background events while keeping a high efficiency for the search modes. This provides for a reduced and manageable event sample to be analyzed, which ultimately increases the speed of the fine tuning procedure herein. The role of the analysis discussed in this chapter 


\section{The Rare Decay Event Selection Algorithm}

is to further tighten the event selection to suppress the background level to as close to zero as possible, while maintaining as high efficiency as possible. This study will be based on MC generated events only and will be applied to the data in Chapter 6 .

Section 5.1 will give the relevant parameters, which are motivated by the signature of the search modes, and sample plots are displayed that show the distributions of these parameters for background events and search modes. From these distributions it is evident that a set of direct cuts can be conveniently applied to enhance the signal to background ratio. The direct cuts are discussed in section 5.2. To finalize the event selection it is neccesary to construct a discriminator function for the individual modes (section 5.3) and the optimal cuts on these discriminator functions are decided upon in section 5.4. In section 5.5 a cross check mode is studied which provides an independent evaluation and control of the methods used in the analysis. Section 5.6 argues for why the use of particle ID is not implemented in the analysis.

\subsection{Parameters}

As the first parameters to be discussed, the vertex mass and the mass of the vector mesons, when relevant, are calculated from the individual tracks which individually must be assigned a kaon mass $(493 \mathrm{MeV}$ ) or a pion mass (140 MeV) depending 


\section{$5.1 \quad$ Parameters}

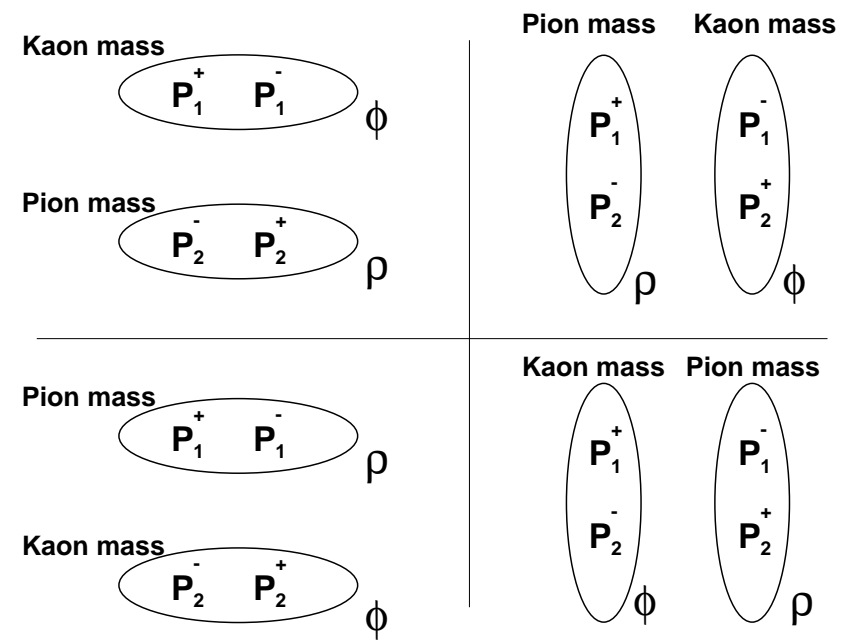

Figure 5.1: The four possible two track combinations are shown for $B^{0} \rightarrow \phi \rho^{0}$. The combination that gives the best reconstructed $\rho$ and $\phi$ masses are picked.

on the mode in question. In section 5.6 it will be explained why the analysis has not implemented the possible particle identifications that can be achieved for pions, kaons and protons in the CRID, muons in the WIC and electrons in the LAC. For each mode then the mass assignment is based upon a combinatorial game where all possible combinations are compared to each other and the one is picked that satisfies a rule depending on the mode.

For the modes $B^{0} \rightarrow \pi^{+} \pi^{-}, B_{s} \rightarrow \pi^{+} \pi^{-}, B^{+} \rightarrow \rho^{0} \pi^{+}, B^{0} \rightarrow \rho^{0} \rho^{0}$ and $B_{s} \rightarrow$ $\rho^{0} \rho^{0}$ this is a trivial task and all the tracks are assigned a pion mass. Similarly for $B^{0} \rightarrow K^{+} K^{-}, B_{s} \rightarrow K^{+} K^{-}, B^{+} \rightarrow \phi K^{+}, B^{0} \rightarrow \phi \phi$ and $B_{s} \rightarrow \phi \phi$, where all the tracks are assigned a kaon mass. The vertex mass is here given and for the modes 


\section{The Rare Decay Event Selection Algorithm}
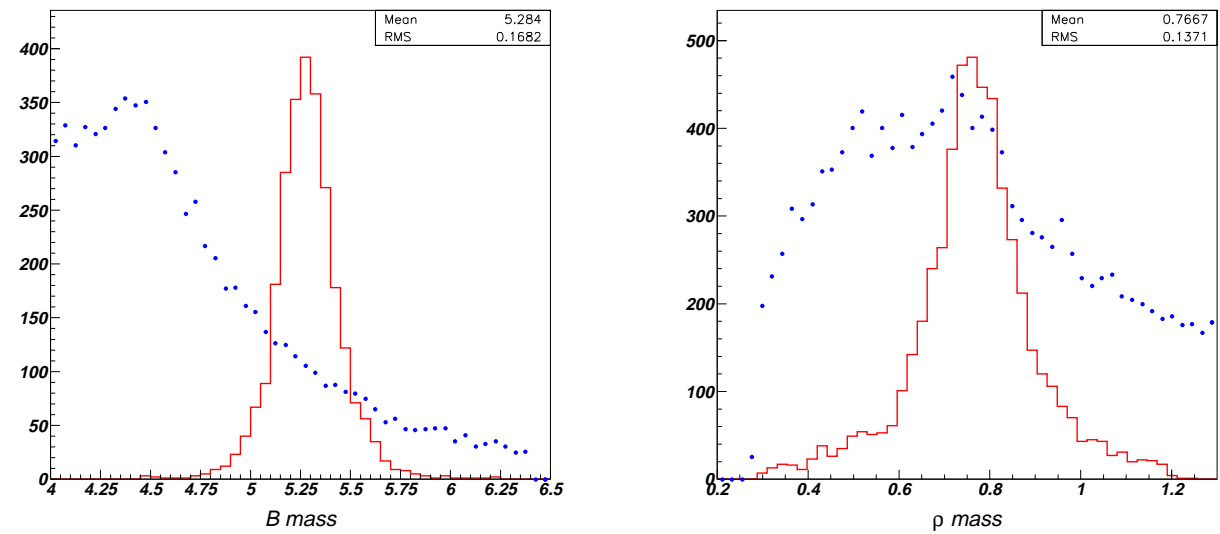

Figure 5.2: Shown are the reconstructed vertex mass and the vector masses for the $B^{+} \rightarrow \rho^{0} \pi^{+}$mode. The dots are the candidate vertices from MC background events and the solid histograms are the candidate vertices from MC generated events of rare $B$ decays. The relative scale between background and signal is arbitrary. The statistics shown are for the signal modes.

that involve a vector meson the reconstructed mass of the vector is taken from the two track combination that closest reproduces the known vector mass. For the modes $B^{0} \rightarrow K^{-} \pi^{+}$and $B_{s} \rightarrow K^{+} \pi^{-}$where one track is a kaon and the other a pion, the track mass assignment that gives a vertex mass closest to $5.28 \mathrm{GeV}\left(B^{0}\right)$ or $5.37\left(B_{s}\right)$ is picked. The remaining modes, all involving at least one vector, have a combination of kaons and pions in the final state. The combination that best reconstructs the vector meson mass(es) is picked. For example, in $B^{0} \rightarrow \phi \rho^{0}$ there are two pions and two kaons in the final state. Two of the tracks have positive charge $P_{1}^{+}, P_{2}^{+}$ and two tracks have negative charge $P_{1}^{-}, P_{2}^{-}$. Figure 5.1 shows the four possible 

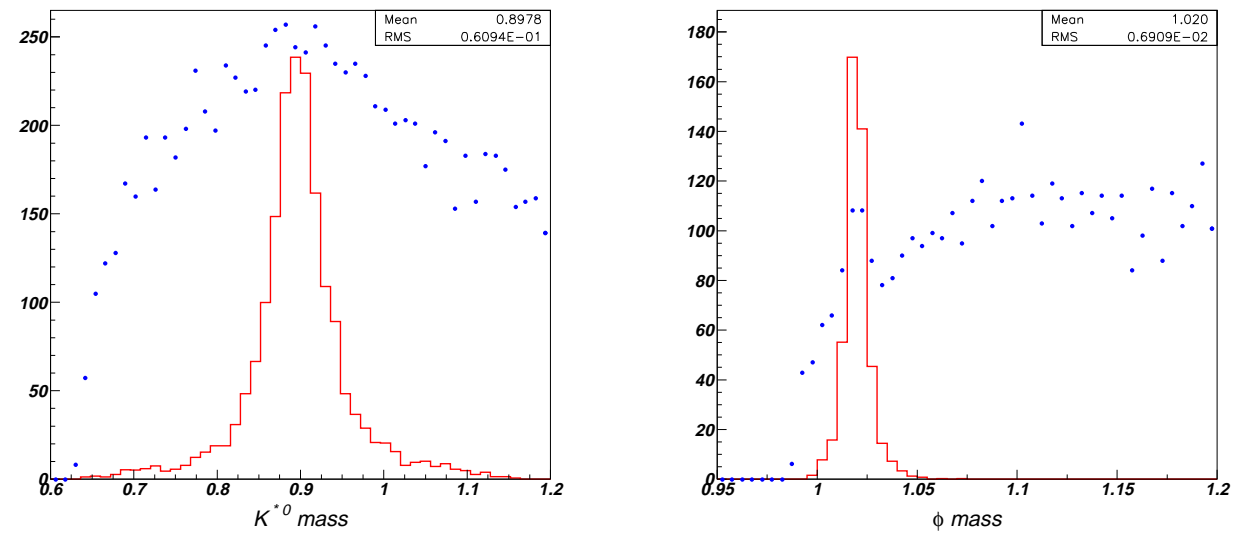

Figure 5.3: Shown are the reconstructed vector masses for the $B^{+} \rightarrow K^{* 0} \pi^{+}$and $B^{+} \rightarrow \phi \pi^{+}$modes. The dots are the candidate vertices from MC background events and the solid histograms are the candidate vertices from MC generated events of rare $B$ decays. The relative scale between background and signal is arbitrary. The statistics shown are for the signal modes.

combinations in this case and the combination that is picked is the minimal solution to the expression $\left|M_{\phi}-m_{\phi}\right|+\left|M_{\rho}-m_{\rho}\right|$ where $m$ denotes the reconstructed mass and $M$ denotes the true mass. The vertex mass $(B)$ is then calculated with the particular track mass assignments that were chosen ${ }^{1}$ as inputs. Figures 5.2 and 5.3 show the reconstructed vertex mass and the vector masses for candidate vertices (Chapter 4) in $B^{+} \rightarrow \rho^{0} \pi^{+}, B^{+} \rightarrow K^{* 0} \pi^{+}$and $B^{+} \rightarrow \phi \pi^{+}$modes. The dots are the MC background events and the solid histograms are the MC generated events of

\footnotetext{
${ }^{1}$ Being much lighter than the $B$ meson, the reconstructed vector masses are more sensitive to the track mass assignments and the reconstructed $B$ mass is not used as a measure for picking the best combination.
} 


\section{The Rare Decay Event Selection Algorithm}
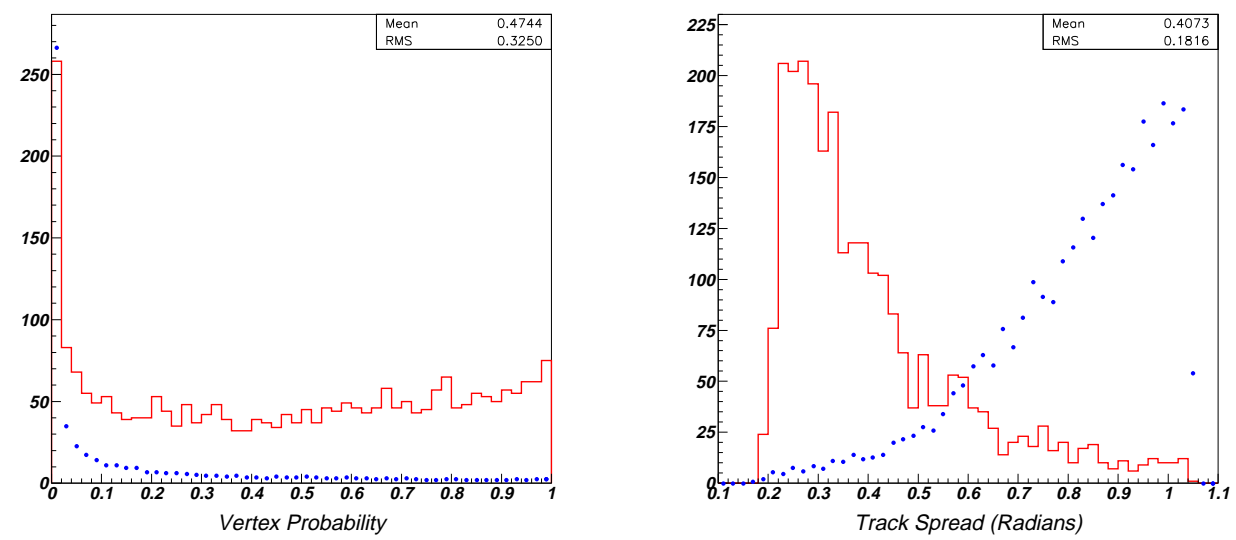

Figure 5.4: Shown are the vertex probability and the largest spread between any tracks in a candidate vertex for the $B^{+} \rightarrow \rho^{0} \pi^{+}$mode. The dots are the candidate vertices from MC background events and the solid histograms are the candidate vertices from MC generated events of rare $B$ decays. The relative scale between background and signal is arbitrary. The statistics shown are for the signal modes.

rare $B$ decays. The relative scale between background and signal is arbitrary. The width $\Gamma$ of the vector masses scales accordingly with their lifetimes $\tau, \Gamma=1 / \tau$, and is not limited by detector resolution.

The vertex probability, the vertex significance, the track spread and the stiffest track momentum have already been discussed in Chapter 4, and Figure 4.5 in the same chapter shows the significance distribution for signal and background. Figure 5.4 shows the vertex probability and the track spread for candidate vertices, background and signal events for the mode $B^{+} \rightarrow \rho^{0} \pi^{+}$. 


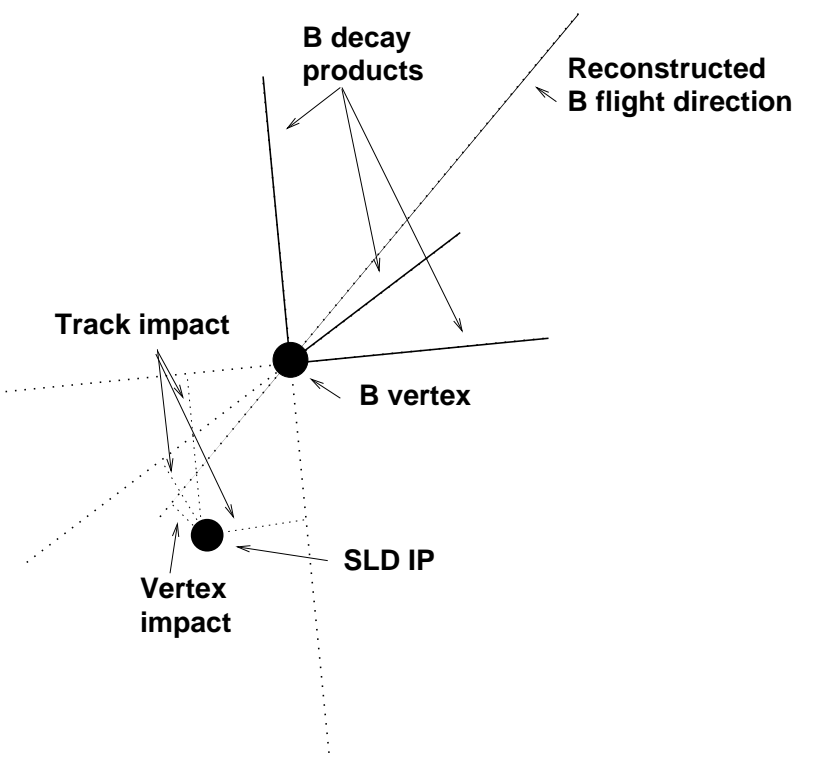

Figure 5.5: Shown are the vertex impact parameter and the track impact parameters. It is expected that the vertex impact parameter is small while the normalized track impact parameters are expected to be large enough to significantly separate them from the SLD IP.

The next two parameters to be discussed are related to the topology of the $B$ vertex. In Figure 4.6 an illustration is drawn of a $B$ meson decaying into a $D$ meson and some other decay products. Although not relevant to the modes herein (which contain no long lived decay products such as the $D$ ) it still shows a general feature of a $B$ decay. First of all, the sum of the decay product momenta should give the $B$ meson flight direction. By extrapolation, one expects this momentum sum to point back to the SLD IP. Secondly, the momenta of the individual tracks is expected not to point back to the SLD IP, unlike fragmentation tracks. This motivates two 


\section{The Rare Decay Event Selection Algorithm}
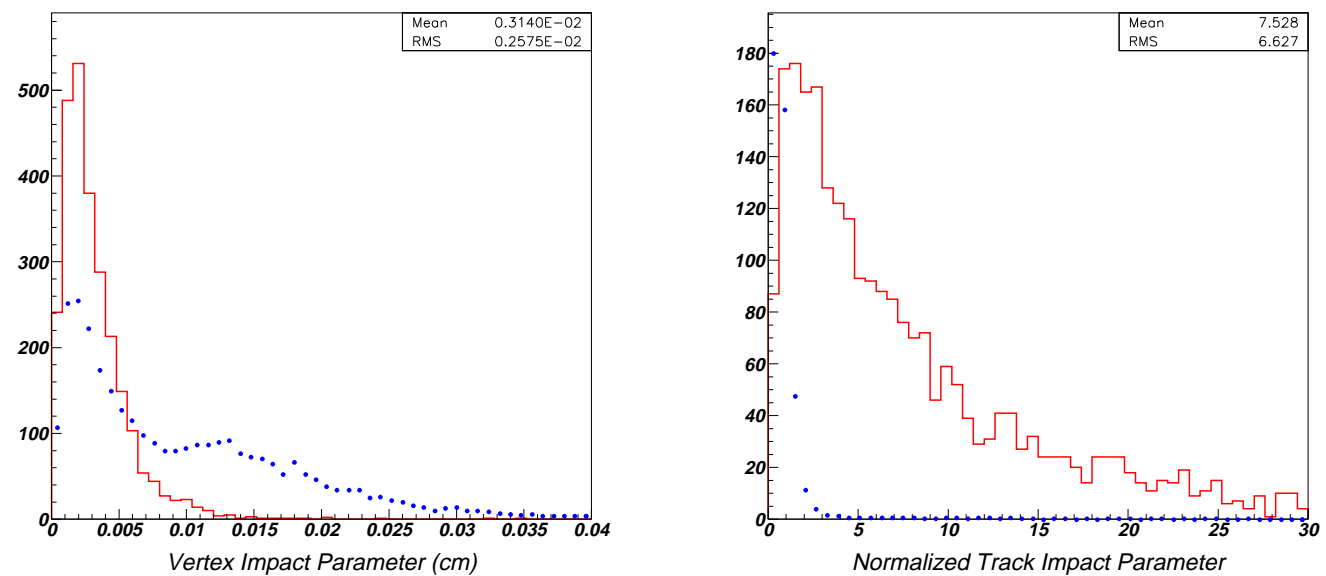

Figure 5.6: Shown are the reconstructed vertex impact parameter and the track impact parameters for the mode $B^{+} \rightarrow \rho^{0} \pi^{+}$. The dots are the candidate vertices from $\mathrm{MC}$ background events and the solid histograms are the candidate vertices from MC generated events of rare $B$ decays. The relative scale between background and signal is arbitrary. The statistics shown are for the signal modes.

parameters, the vertex impact parameter and the track impact parameter. Figure

5.5 shows a cartoon where these two parameters are illustrated. From now, when

the track impact parameter is mentioned, what is meant is the smallest normalized impact parameter of all the tracks in a candidate vertex. Figure 5.6 shows the track impact parameter and the vertex impact parameter for signal and background as found in MC events.

A special property is seen in $B \rightarrow P V$, where the vector meson (spin 1 ) is transverse polarized to preserve angular momentum ( $B$ and $P$ are spin 0 states). 

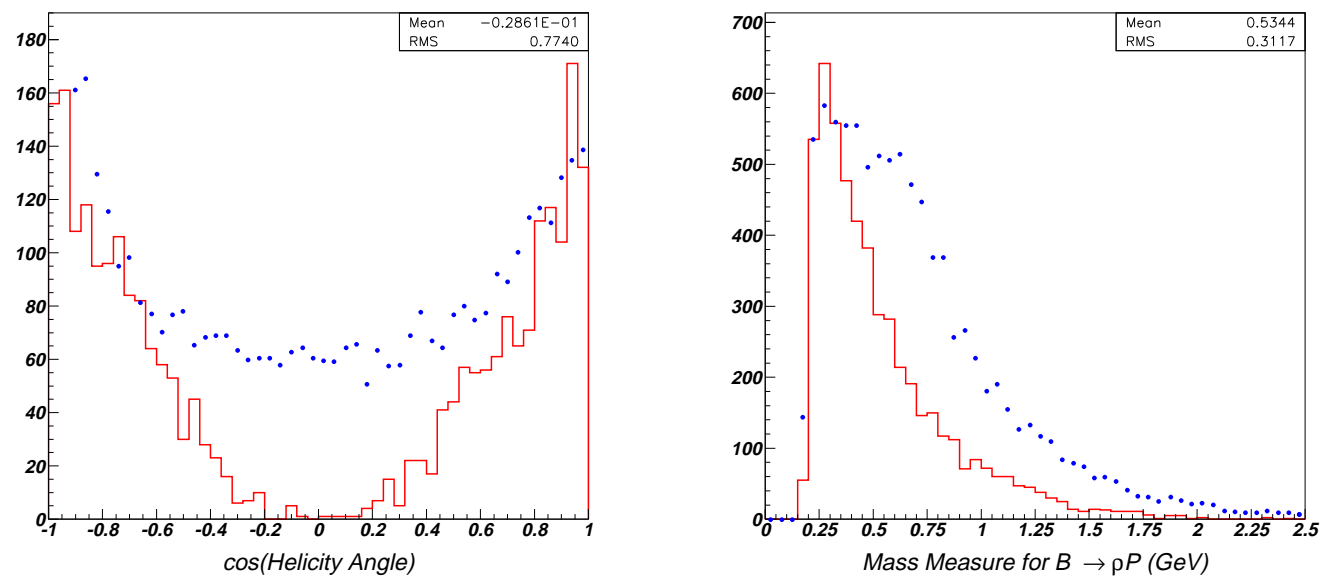

Figure 5.7: Shown are the reconstructed helicity angle and the mass measure parameters for the mode $B^{+} \rightarrow \rho^{0} \pi^{+}$. The dots are the candidate vertices from $\mathrm{MC}$ background events and the solid histograms are the candidate vertices from MC generated events of rare $B$ decays. The relative scale between background and signal is arbitrary. The statistics shown are for the signal modes.

The helicity angle is defined as the angle between the flight direction of the vector $V$ and the direction of its decay particles in the vector rest frame. Due to the transverse polarization of the vector meson the helicity angle $\theta_{h}$ follows a $\cos \theta_{h}$ distribution. Figure 5.7 shows a plot of the reconstructed helicity angle for signal and background. Also, shown are a mass measure parameter for $B \rightarrow P V$. This is the reconstructed vertex mass if all tracks are assigned a kaon mass minus the reconstructed vertex mass if all tracks are assigned a pion mass. A similar mass measure is implemented for $B \rightarrow P P$ where this mass measure is calculated for the modes that have at 


\section{4}

\section{The Rare Decay Event Selection Algorithm}

least one final kaon track. It is the correctly reconstructed vertex mass minus the reconstructed vertex mass if all tracks are assigned a pion mass. For $B \rightarrow P P$ and $B \rightarrow P V$ modes the mass measures exploit the sensitivity of the reconstructed vertex masses to the individual track mass assignments, and provide a parameter to suppress background events that artificially get a large vertex mass due to kaon assignments to the tracks.

For $B \rightarrow V V$ a different mass measure is constructed. It is the quantity $\mid M_{v 1}-$ $m_{v 1}|+| M_{v 2}-m_{v 2}|+| M_{B}-m_{B} \mid$, where capital $\mathrm{M}$ is the known mass of the vectors $(v 1, v 2)$ and the vertex $(B)$, and small $m$ is the corresponding reconstructed masses. This parameter measures the overall performance of the combined reconstructed vector and vertex masses. Figure 5.8 shows the mass measures for $B^{0} \rightarrow K^{+} K^{-}$and $B^{0} \rightarrow \bar{K}^{* 0} \rho^{0}$.

\section{$5.2 \quad$ Direct cuts}

It should be evident from the previous section that a set of hard cuts can be implemented in such a way that the signal to background ratio will be strongly enhanced. On the other hand, it is not possible to remove all the background with direct cuts while keeping a large signal. The direct cuts listed here are a compromise 


\subsection{Direct cuts}
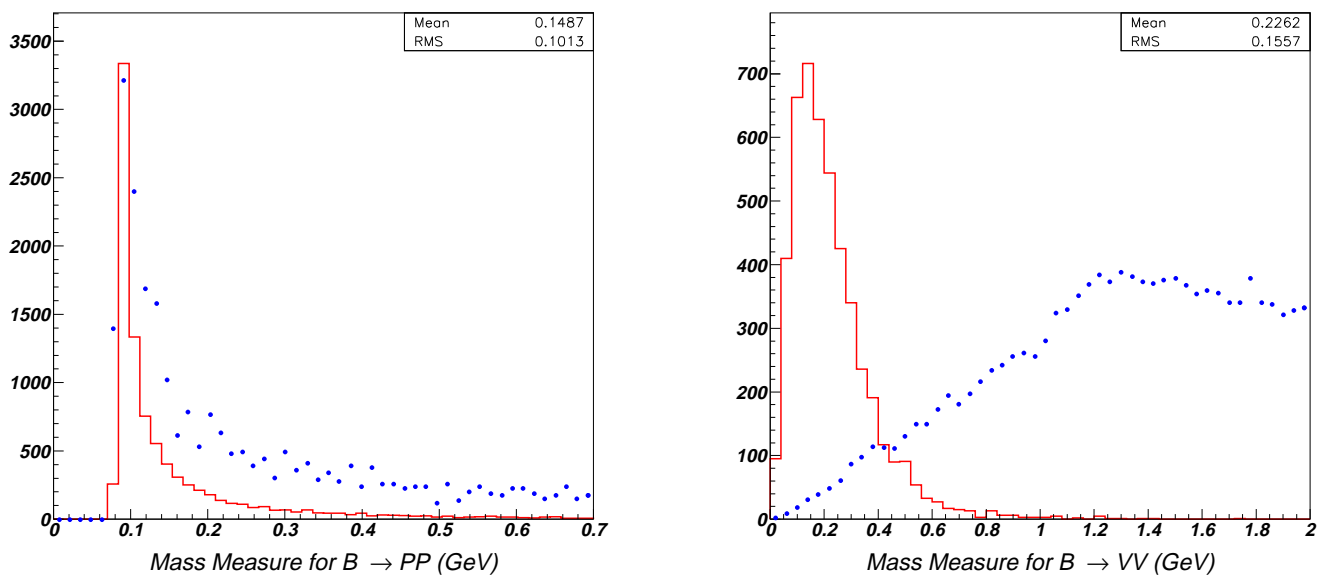

Figure 5.8: Shown are the mass measure parameters for the modes $B^{0} \rightarrow K^{+} K^{-}$and $B_{s} \rightarrow \bar{K}^{* 0} \rho^{0}$ as explained in the text. The dots are the candidate vertices from MC background events and the solid histograms are the candidate vertices from MC generated events of rare $B$ decays. The relative scale between background and signal is arbitrary. The statistics shown are for the signal modes.

between stiff cuts which would remove all the background versus the desire to keep the efficiency as high as possible. There will to some extent be correlation between the various parameters and each of the direct cuts are not necessarily set at an optimal point by themselves. The tuning of the direct cuts together with a discriminator function (to be described later) was done in an adhoc way to best separate signal from background. The overall performance has dictated the decision on which direct cuts to make.

All modes require that the reconstructed vertex mass, after the individual track 


\section{The Rare Decay Event Selection Algorithm}

mass assignment, is larger than $5.05 \mathrm{GeV}$ and $5.15 \mathrm{GeV}$ for $B^{+}, B^{0}$ modes and $B_{s}$ modes respectively. The other direct cuts are mode dependent.

- The direct cuts for $B \rightarrow P P$ modes require the vertex fit probability $P$ to be larger than $1 \%$ and the vertex significance $S$ to be larger than 1.0. It further includes a cut on the normalized track impact parameter $B$ which must be larger than 1.1. In addition, for the modes that have at least one track assigned a kaon mass, the reconstructed mass measure $\mathcal{M}$ cannot be larger than 300 $\mathrm{MeV}$.

- The direct cuts for the $B \rightarrow P V$ includes a cut on the reconstructed mass of the vector candidate $(\mathrm{V})$. The allowed ranges are $[0.2,1.1] \mathrm{GeV},[0.7,1.0] \mathrm{GeV}$ and $[1.000,1.035] \mathrm{GeV}$ for $\rho^{0}, K^{* 0}$ and $\phi$ respectively. Further, the helicity angle $\theta_{h}$ cut is given by $\left|\cos \left(\theta_{h}\right)\right|>0.3$. For the $B \rightarrow P V$ modes excluding the combination with a $\phi$ we apply the same cuts on the vertex probability (1\%), vertex significance (1.0) and normalized track impact parameter (1.1) as for the $B \rightarrow P P$ modes. For the $B \rightarrow P V$ mode where the vector particle is $\phi$ the vertex probability must be larger than $0.5 \%$ and the normalized impact parameter must be larger than 0.6. Here, the vertex significance cut is left untouched from the 0.6 cut applied in forming candidate vertices. For modes 


\subsection{Direct cuts}

\begin{tabular}{lccccc}
\hline Mode & $P$ & $S$ & $B$ & $\mathcal{M}$ & $|\cos h|$ \\
\hline$B \rightarrow P P$ & $>1 \%$ & $>1.0$ & $>1.1$ & $\leq 300 \mathrm{MeV}$ & \\
$B \rightarrow P \rho^{0}$ & $>1 \%$ & $>1.0$ & $>1.1$ & $\leq 1.2 \mathrm{GeV}$ & $>0.3$ \\
$B \rightarrow P K^{* 0}$ & $>1 \%$ & $>1.0$ & $>1.1$ & & $>0.3$ \\
$B \rightarrow P \phi$ & $>0.5 \%$ & $>0.6$ & $>0.6$ & & $>0.3$ \\
$B \rightarrow \rho \rho$ & $>0.5 \%$ & $>0.6$ & $>0.6$ & $<0.6 \mathrm{GeV}$ & \\
$B \rightarrow \rho V, K^{* 0} \bar{K}^{* 0}$ & $>0.5 \%$ & $>0.6$ & $>0.6$ & $<0.4 \mathrm{GeV}$ & \\
$B \rightarrow \phi \phi$ & $>0.5 \%$ & $>0.6$ & $>0.6$ & & \\
\hline Mass Cuts $(\mathrm{GeV})$ & $m_{\rho}$ & $m_{K^{* o}}$ & $m_{\phi}$ & $m_{B_{u, d}}$ & $m_{B_{s}}$ \\
\hline & $0.2-1.1$ & $0.7-1.0$ & $1.000-1.035$ & $>5.05$ & $>5.15$ \\
\hline
\end{tabular}

Table 5.1: Listed are the direct cuts as explained in the text.

involving a $\rho^{0}$, the mass measure must be less than $1.2 \mathrm{Gev}$.

- For the $B \rightarrow V V$ modes the vertex probability must be larger than $0.5 \%$ and the normalized impact parameter must be larger than 0.6 . The vertex significance cut is kept at the original 0.6 value. The allowed range for the reconstructed vector masses are the same as for the $B \rightarrow P V$ modes, $[0.2,1.1]$ $\mathrm{GeV},[0.7,1.0] \mathrm{GeV}$ and $[1.000,1.035] \mathrm{GeV}$ for $\rho^{0}, K^{* 0}$ and $\phi$ respectively. In the case of two $\rho^{0}$ 's the mass measure quantity must be less than $0.6 \mathrm{GeV}$, and in the case of one $\rho^{0}$ and either a $K^{* 0}$ or a $\phi$ this quantity must be less than $0.4 \mathrm{GeV}$, which is also the limit for two $K^{* 0}$ 's. Other $B \rightarrow V V$ combinations do not apply this mass measure cut.

This completes the list of the hard cuts and in Table 5.1 a summary is given. They 

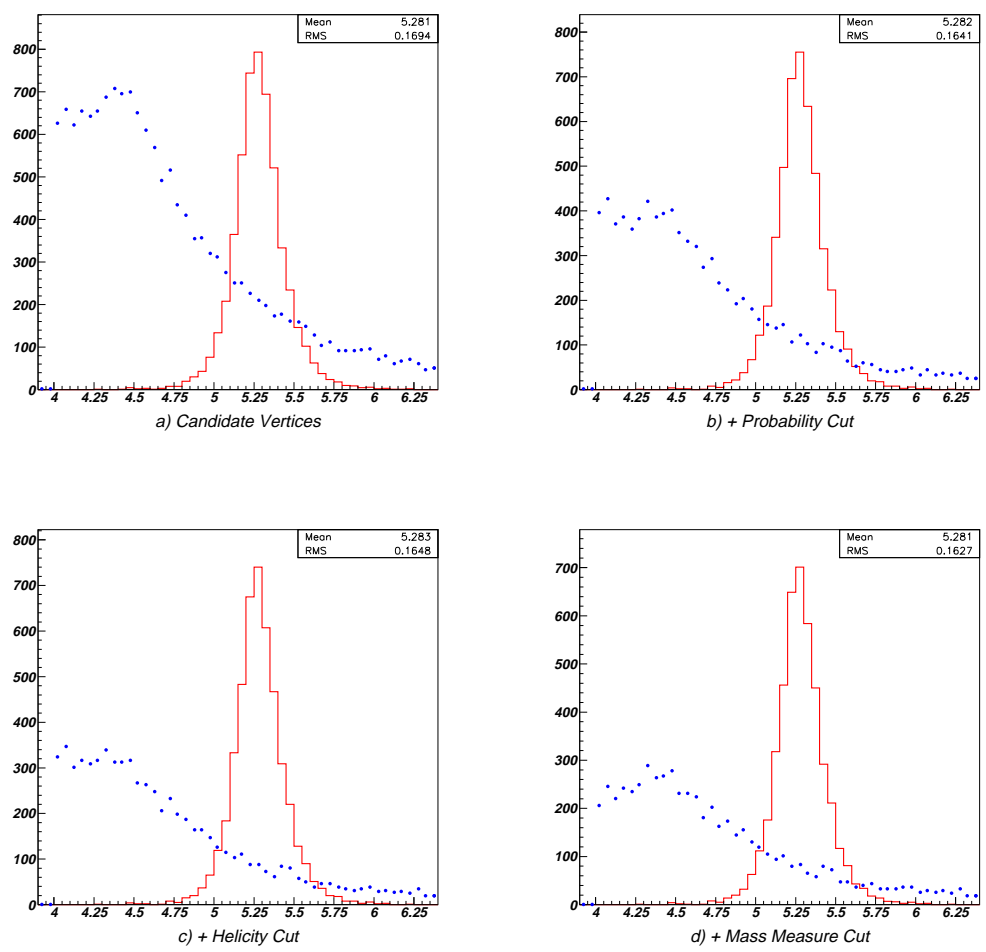

Figure 5.9: Shown are the evolution of the mass distribution for the $B^{+} \rightarrow \rho^{0} \pi^{+}$mode when the direct cuts are added for MC background (dots) and MC signal (solid). The statistics shown are for the signal events. The entries in plot a) are the candidate vertices with a non specified relative weight between signal and background. Cuts are added including plot h) (Figure 5.10).

reduce the combinatorial background significantly while leaving a large portion of the desired modes intact. The effect of the direct cuts varies from mode to mode and while the $B \rightarrow \phi \phi$ mode has no background left after these cuts other modes still need additional ways to separate background from signal. As an example of the effect of these direct cuts, Figures 5.9 and 5.10 shows the evolution of the mass 


\subsection{Direct cuts}
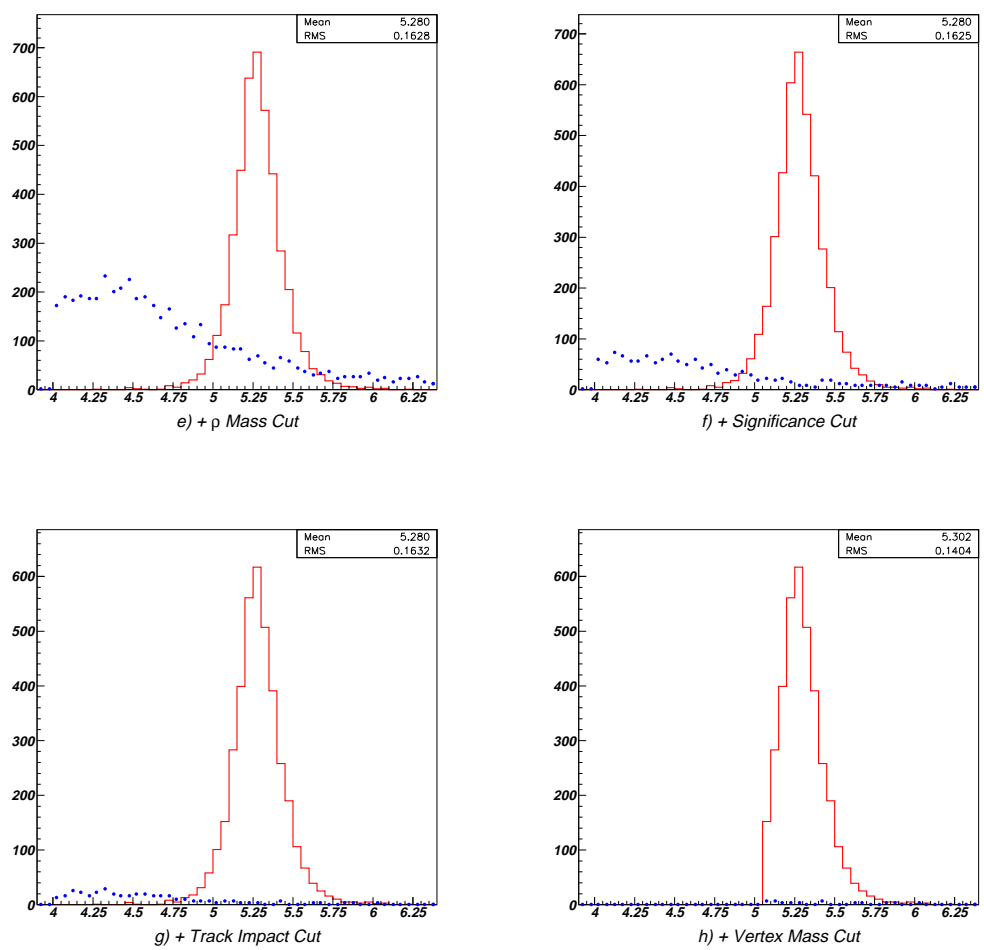

Figure 5.10: Shown are the evolution of the mass distribution for the $B^{+} \rightarrow \rho^{0} \pi^{+}$mode when the different direct cuts are added. The dots represents the $\mathrm{MC}$ background and the solid curves are the MC signal. The statistics shown are for the signal events. The entries in plot a) (Figure 5.9) are the candidate vertices with a non specified relative weight between signal and background.

distribution for the $B^{+} \rightarrow \rho^{0} \pi^{+}$mode when the various cuts are added. Figure a) shows the candidate vertices as defined in Chapter 4, b) is the mass distribution when the vertex probability cut at $1 \%$ is applied, c) is the mass distribution after the helicity $\operatorname{cut}\left(\left|\cos \left(\theta_{h}\right)\right|>0.3\right)$ is added, d) after the mass measure cut at $1.2 \mathrm{GeV}$, e) after the vector mass cut $\left.\left(m_{\rho} \in[0.2,1.1] \mathrm{GeV}\right), \mathrm{f}\right)$ after the vertex significance 
The Rare Decay Event Selection Algorithm

\begin{tabular}{||l|c|c|c|c||l|c|c|c|c||}
\hline \hline Mode & \multicolumn{2}{|c|}{ VXD2 } & \multicolumn{2}{|c||}{ VXD3 } & Mode & \multicolumn{2}{c||}{ VXD2 } & \multicolumn{2}{c||}{ VXD3 } \\
\hline & Bck. & $\epsilon \%$ & Bck. & $\epsilon \%$ & & Bck. & $\epsilon \%$ & Bck. & $\epsilon \%$ \\
\hline$B^{0} \rightarrow \pi^{+} \pi^{-}$ & 6.1 & 35 & 6.5 & 39 & $B^{0} \rightarrow \rho^{0} \rho^{0}$ & 43 & 33 & 24 & 35 \\
$B^{0} \rightarrow K^{-} \pi^{+}$ & 7.7 & 35 & 7.8 & 38 & $B^{0} \rightarrow \bar{K}^{* 0} \rho^{0}$ & 54 & 29 & 31 & 32 \\
$B^{0} \rightarrow K^{+} K^{-}$ & 6.9 & 34 & 8.9 & 40 & $B^{0} \rightarrow \bar{K}^{* 0} K^{* 0}$ & 52 & 32 & 26 & 36 \\
$B_{s} \rightarrow \pi^{+} \pi^{-}$ & 6.4 & 37 & 6.7 & 43 & $B^{0} \rightarrow \phi \rho^{0}$ & 9.7 & 28 & 3.3 & 34 \\
$B_{s} \rightarrow K^{+} \pi^{-}$ & 7.1 & 37 & 7.3 & 40 & $B^{0} \rightarrow \phi \bar{K}^{* 0}$ & 10 & 28 & 4.5 & 33 \\
$B_{s} \rightarrow K^{+} K^{-}$ & 6.1 & 33 & 7.7 & 35 & $B^{0} \rightarrow \phi \phi$ & 0.0 & 26 & 0.1 & 32 \\
\hline$B^{+} \rightarrow \rho^{0} \pi^{+}$ & 16 & 30 & 9.8 & 34 & $B_{s} \rightarrow \rho^{0} \rho^{0}$ & 37 & 30 & 20 & 36 \\
$B^{+} \rightarrow \rho^{0} K^{+}$ & 16 & 27 & 11 & 34 & $B_{s} \rightarrow \bar{K}^{* 0} \rho^{0}$ & 49 & 31 & 28 & 34 \\
$B^{+} \rightarrow K^{* 0} \pi^{+}$ & 11 & 30 & 8.6 & 34 & $B_{s} \rightarrow \bar{K}^{* 0} K^{* 0}$ & 46 & 29 & 24 & 35 \\
$B^{+} \rightarrow K^{* 0} K^{+}$ & 11 & 25 & 8.6 & 30 & $B_{s} \rightarrow \phi \rho^{0}$ & 9.1 & 28 & 4.2 & 34 \\
$B^{+} \rightarrow \phi \pi^{+}$ & 12 & 31 & 11 & 34 & $B_{s} \rightarrow \phi K^{* 0}$ & 9.0 & 27 & 4.7 & 29 \\
$B^{+} \rightarrow \phi K^{+}$ & 13 & 31 & 13 & 36 & $B_{s} \rightarrow \phi \phi$ & 0.1 & 25 & 0.0 & 34 \\
\hline \hline
\end{tabular}

Table 5.2: Shown are the expected background levels (number of events) and the expected efficiencies after the direct cuts are applied. The VXD2 and the VXD3 running periods are treated separately due to different tracking performance with the two vertex detectors.

cut at $1, \mathrm{~g})$ after the normalized track impact parameter cut at 1.1 and $\mathrm{h}$ ) finally the vertex mass cut.

Table 5.2 contains a comprehensive list of expected background levels and efficiencies after these cuts. The VXD2 and the VXD3 running period are displayed separately to show the improved performance with the VXD3. It can be seen that the VXD3 period in general gives higher efficiencies while the background level is about the same or less. This is significant given the larger statistics available for VXD3 and it motivates splitting the remaining event selection into the two run periods. 


\subsection{Discriminator Functions}

\subsection{Discriminator Functions}

A discriminator function is constructed for the individual modes to further improve the event selection. It intends to take advantage of the combined phase space of the signal and background events. Its form is motivated by the distributions of the parameters used in the event selection and the need of a large degree of freedom in choosing the relative weight of the parameters. The Fisher discriminator function [32] which is widely used in the literature requires the various parameters to have a close to Gaussian distribution and limits the set of parameters that can be included, and is not suitable for this analysis ${ }^{2}$. Another widely used method is the maximum likelihood method [33] which limits the possibility of tuning the the relative weights between the parameters. Hence, a totally empirical function is used, the form of which is decided by what works. The tuning of this function is done separately for the VXD2 and VXD3 MC generated events for signal and background. It is a function $\mathcal{F}$ of a set of reconstructed vertex parameters and takes the following form for $B \rightarrow P P$

$$
\begin{aligned}
\mathcal{F}_{P P}= & a_{0} e^{-\frac{\left(m_{b}-M_{b}\right)^{2}}{2\left(\delta m_{b}\right)^{2}}}-a_{1} e^{\frac{-m_{b}}{m_{1}}}-a_{2} e^{-\frac{S}{3}}-a_{3} e^{-\tilde{\lambda}}+a_{4} e^{-\frac{\lambda}{0.3}} \\
& -a_{5} e^{-\frac{P}{0.03}}-a_{6} e^{-\frac{B}{3}}-a_{7} e^{-\frac{\tilde{I}}{I_{0}}}+a_{8} e^{-\frac{I}{I_{0}}}-a_{9} e^{-\frac{X}{0.5}}+a_{10} e^{-\frac{\Delta M}{0.2 \mathrm{GeV}}},
\end{aligned}
$$

\footnotetext{
${ }^{2}$ The Fisher disriminator function does take advantage of correlations between parameters, while the method used herein does lose explicit information of such correlations.
} 
152

The Rare Decay Event Selection Algorithm

\begin{tabular}{|l|cccccccccccc|}
\hline \hline & $a_{0}$ & $a_{1}$ & $a_{2}$ & $a_{3}$ & $a_{4}$ & $a_{5}$ & $a_{6}$ & $a_{7}$ & $a_{8}$ & $a_{9}$ & $a_{10}$ & $\delta m_{b}$ \\
\hline $\mathrm{VXD} 2$ & & & & & & & & & & & & \\
\hline$B^{0} \rightarrow \pi^{+} \pi^{-}$ & 7 & 2 & 11 & 7 & 4 & 2 & 5 & 10 & 3 & 3 & 0 & 0.15 \\
$B^{0} \rightarrow K^{-} \pi^{+}$ & 10 & 4 & 14 & 10 & 9 & 0 & 9 & 10 & 3 & 3 & 0 & 0.15 \\
$B^{0} \rightarrow K^{+} K^{-}$ & 11 & 2 & 10 & 7 & 8 & 0 & 4 & 10 & 6 & 3 & 2 & 0.16 \\
$B_{s} \rightarrow \pi^{+} \pi^{-}$ & 14 & 2 & 10 & 10 & 9 & 0 & 8 & 10 & 3 & 3 & 0 & 0.15 \\
$B_{s} \rightarrow K^{+} \pi^{-}$ & 14 & 3 & 9 & 13 & 7 & 0 & 14 & 5 & 3 & 3 & 0 & 0.13 \\
$B_{s} \rightarrow K^{+} K^{-}$ & 15 & 3 & 7 & 13 & 8 & 0 & 9 & 4 & 9 & 4 & 0 & 0.12 \\
\hline $\mathrm{VXD} 3$ & & & & & & & & & & & & \\
\hline$B^{0} \rightarrow \pi^{+} \pi^{-}$ & 16 & 3 & 10 & 17 & 6 & 0 & 13 & 6 & 3 & 3 & 0 & 0.10 \\
$B^{0} \rightarrow K^{-} \pi^{+}$ & 12 & 10 & 12 & 15 & 2 & 3 & 11 & 9 & 3 & 3 & 0 & 0.10 \\
$B^{0} \rightarrow K^{+} K^{-}$ & 11 & 2 & 10 & 7 & 8 & 0 & 4 & 10 & 6 & 3 & 2 & 0.16 \\
$B_{s} \rightarrow \pi^{+} \pi^{-}$ & 13 & 2 & 14 & 19 & 5 & 1 & 9 & 14 & 3 & 3 & 0 & 0.15 \\
$B_{s} \rightarrow K^{+} \pi^{-}$ & 14 & 1 & 15 & 16 & 6 & 0 & 11 & 9 & 3 & 3 & 0 & 0.12 \\
$B_{s} \rightarrow K^{+} K^{-}$ & 17 & 2 & 10 & 11 & 7 & 0 & 14 & 8 & 2 & 3 & 0 & 0.12 \\
\hline
\end{tabular}

Table 5.3: A list of the parameters that are used in the discriminator functions for $B \rightarrow$ $P P$

where the reconstructed vertex parameters are as follows. $m_{b}$ is the vertex mass, $S$ is the vertex significance parameter, $\lambda$ is the largest angle between any tracks belonging to the vertex $(\tilde{\lambda}=1 / \lambda-0.9), P$ is the vertex probability from the vertex fit, $B$ is the track impact parameter, $I$ is the vertex impact parameter $(\tilde{I} \times 1000+24=$ $1 /(I+0.001)), X$ is the vertex fragmentation parameter and finally $\Delta M$ is the difference in the reconstructed vertex mass when all particles are assigned a pion mass and when all particles are assigned a kaon mass. The rest of the variables are parameters which are tuned for the individual modes. Terms that come in with a minus sign in front of the coefficients $a_{i}$ generally suppress the background while 


\subsection{Discriminator Functions}

\begin{tabular}{|l|ccccccccccc|}
\hline \hline & $a_{0}$ & $a_{1}$ & $a_{2}$ & $a_{3}$ & $a_{4}$ & $a_{5}$ & $a_{6}$ & $a_{7}$ & $a_{8}$ & $a_{9}$ & $a_{11}$ \\
\hline VXD2 & & & & & & & & & & & \\
\hline$B^{+} \rightarrow \rho^{0} \pi^{+}$ & 12 & 4 & 10 & 17 & 3 & 0 & 20 & 11 & 6 & 0 & 13 \\
$B^{+} \rightarrow \rho^{0} K^{+}$ & 12 & 4 & 10 & 17 & 3 & 0 & 20 & 11 & 6 & 0 & 13 \\
$B^{+} \rightarrow K^{* 0} \pi^{+}$ & 8 & 10 & 6 & 15 & 3 & 5 & 22 & 25 & 3 & 2 & 9 \\
$B^{+} \rightarrow K^{* 0} K^{+}$ & 8 & 10 & 6 & 15 & 3 & 5 & 22 & 25 & 3 & 2 & 9 \\
$B^{+} \rightarrow \phi \pi^{+}$ & 9 & 10 & 10 & 20 & 3 & 5 & 10 & 18 & 3 & 2 & 10 \\
$B^{+} \rightarrow \phi K^{+}$ & 13 & 10 & 13 & 19 & 4 & 5 & 13 & 18 & 3 & 2 & 9 \\
\hline$V^{+} 3$ & & & & & & & & & & & \\
\hline$B^{+} \rightarrow \rho^{0} \pi^{+}$ & 11 & 7 & 4 & 15 & 6 & 0 & 4 & 25 & 2 & 0 & 10 \\
$B^{+} \rightarrow \rho^{0} K^{+}$ & 6 & 10 & 2 & 14 & 2 & 0 & 4 & 25 & 2 & 0 & 8 \\
$B^{+} \rightarrow K^{* 0} \pi^{+}$ & 11 & 10 & 5 & 14 & 4 & 8 & 5 & 27 & 3 & 1 & 10 \\
$B^{+} \rightarrow K^{* 0} K^{+}$ & 11 & 10 & 5 & 14 & 4 & 8 & 5 & 27 & 3 & 1 & 10 \\
$B^{+} \rightarrow \phi \pi^{+}$ & 10 & 8 & 10 & 20 & 3 & 3 & 10 & 15 & 3 & 2 & 10 \\
$B^{+} \rightarrow \phi K^{+}$ & 12 & 7 & 10 & 18 & 4 & 3 & 10 & 15 & 3 & 2 & 10 \\
\hline \hline
\end{tabular}

Table 5.4: A list of the parameters that are used in the discriminator functions for $B \rightarrow$ $P V$.

terms with a positive sign enhance the signal. The vertex mass $M_{b}$ is set at the known values, $5.28 \mathrm{GeV}$ for $B^{0}$ or $B^{+}$and $5.37 \mathrm{GeV}$ for $B_{s}$. For all $B \rightarrow P P$ modes $m_{1}=6 \mathrm{GeV}, \tilde{I}_{0}=0.05 \mathrm{~cm}^{-1}$ and $I_{0}=0.005 \mathrm{~cm}$. Table 5.3 shows the remaining parameters.

For $B \rightarrow P V$, the discriminator function looks like

$$
\mathcal{F}_{P V}=\left.\mathcal{F}_{P P}\right|_{a_{10=0}}+a_{11} e^{-\frac{\left(m_{v}-M_{v}\right)^{2}}{2\left(\delta m_{v}\right)^{2}}}+(1-\cos (h \pi))
$$

where $m_{v}$ is the reconstructed mass of the relevant vector and $h$ is the $\cos \left(\theta_{h}\right)$ with $\theta_{h}$ being the reconstructed helicity angle. The known vector masses $M_{v}$ equals 0.77 $\mathrm{GeV}, 0.89 \mathrm{GeV}$ and $1.02 \mathrm{GeV}$ for the $\rho, K^{* 0}$ and $\phi$ respectively. The vector mass 
154

The Rare Decay Event Selection Algorithm

\begin{tabular}{|c|c|c|c|c|c|c|c|c|c|c|c|c|c|}
\hline & $a_{0}$ & $a_{1}$ & $a_{2}$ & $a_{3}$ & $a_{4}$ & $a_{5}$ & $a_{6}$ & $a_{7}$ & $a_{8}$ & $a_{9}$ & $a_{11}$ & $a_{12}$ & $a_{13}$ \\
\hline \multicolumn{14}{|l|}{ VXD2 } \\
\hline$B^{0} \rightarrow \rho^{0} \rho^{0}$ & 8 & 10 & 7 & 28 & 5 & 7 & 15 & 16 & 4 & 1 & 5 & 5 & 8 \\
\hline$B^{0} \rightarrow \bar{K}^{* 0} \rho^{0}$ & 10 & 9 & 10 & 32 & 3 & 6 & 25 & 12 & 2 & 1 & 5 & 9 & 8 \\
\hline$B^{0} \rightarrow \bar{K}^{* 0} K^{* 0}$ & 10 & 9 & 9 & 32 & 3 & 6 & 22 & 11 & 2 & 1 & 7 & 7 & 8 \\
\hline$B^{0} \rightarrow \phi \rho^{0}$ & 10 & 9 & 9 & 28 & 5 & 0 & 20 & 10 & 2 & 1 & 7 & 10 & 8 \\
\hline$B^{0} \rightarrow \phi \bar{K}^{\star 0}$ & 10 & 8 & 10 & 28 & 6 & 1 & 20 & 10 & 2 & 1 & 9 & 11 & 8 \\
\hline$B^{0} \rightarrow \phi \phi$ & 10 & 8 & 10 & 28 & 6 & 1 & 20 & 10 & 2 & 1 & 11 & 11 & 8 \\
\hline \multicolumn{14}{|l|}{ VXD3 } \\
\hline$B^{0} \rightarrow \rho^{0} \rho^{0}$ & 8 & 12 & 14 & 25 & 4 & 3 & 14 & 14 & 5 & 1 & 5 & 5 & 8 \\
\hline$B^{0} \rightarrow \bar{K}^{* 0} \rho^{0}$ & 10 & 9 & 8 & 28 & 3 & 6 & 23 & 12 & 2 & 0 & 5 & 9 & 8 \\
\hline$B^{0} \rightarrow \bar{K}^{* 0} K^{* 0}$ & 10 & 9 & 9 & 29 & 3 & 6 & 22 & 10 & 2 & 1 & 7 & 7 & 8 \\
\hline$B^{0} \rightarrow \phi \rho^{0}$ & 11 & 8 & 9 & 24 & 6 & 0 & 18 & 10 & 2 & 1 & 7 & 10 & 9 \\
\hline$B^{0} \rightarrow \phi \bar{K}^{\star 0}$ & 10 & 8 & 10 & 28 & 6 & 1 & 20 & 10 & 2 & 1 & 9 & 11 & 8 \\
\hline$B^{0} \rightarrow \phi \phi$ & 10 & 8 & 10 & 28 & 6 & 1 & 20 & 10 & 2 & 1 & 11 & 11 & 8 \\
\hline
\end{tabular}

Table 5.5: A list of the parameters that are used in the discriminator functions for $B^{0} \rightarrow$ $V V$

widths are further set to $\delta m_{\rho}=0.1 \mathrm{GeV}, \delta m_{K^{* 0}}=0.08 \mathrm{GeV}$ and $\delta m_{\phi}=0.006 \mathrm{GeV}$ and the vertex mass width $\delta m_{b}$ is set to $=0.1 \mathrm{Gev}$ for the modes involving a $\rho$ and it is set to $0.08 \mathrm{GeV}$ for modes involving a $K^{* 0}$ or a $\phi$. For modes excluding the ones with a $\rho$ vector, $m_{1}=6 \mathrm{GeV}, \tilde{I}_{0}=0.1 \mathrm{~cm}^{-1}$ and $I_{0}=0.04 \mathrm{~cm}$. They are $m_{1}=5$ $\mathrm{GeV}, \tilde{I}_{0}=0.05 \mathrm{~cm}^{-1}$ and $I_{0}=0.005 \mathrm{~cm}$ for modes with a $\rho$ vector. Table 5.4 shows the remaining parameters.

For $B \rightarrow V V$, the discriminator function looks like

$$
\mathcal{F}_{V V}=\left.\mathcal{F}_{P P}\right|_{a_{10=0}}+a_{11} e^{-\frac{\left(m_{v}^{(1)}-M_{v}^{(1)}\right)^{2}}{2\left(\delta m_{v}^{(1)}\right)^{2}}}+a_{12} e^{-\frac{\left(m_{v}^{(2)}-M_{v}^{(2)}\right)^{2}}{2\left(\delta m_{v}^{(2)}\right)^{2}}}+a_{13} e^{-\frac{\mathcal{M}}{0.4 \mathrm{GeV} V}},
$$

where $m_{v}^{(i)}$ is the reconstructed mass of vector $i$, ordered such that $m_{v}^{(1)} \leq m_{v}^{(2)}$ and 


\subsection{Discriminator Functions}

\begin{tabular}{|l|ccccccccccccc|}
\hline \hline & $a_{0}$ & $a_{1}$ & $a_{2}$ & $a_{3}$ & $a_{4}$ & $a_{5}$ & $a_{6}$ & $a_{7}$ & $a_{8}$ & $a_{9}$ & $a_{11}$ & $a_{12}$ & $a_{13}$ \\
\hline $\mathrm{VXD} 2$ & & & & & & & & & & & & & \\
\hline$B_{s} \rightarrow \rho^{0} \rho^{0}$ & 8 & 10 & 7 & 28 & 5 & 7 & 15 & 16 & 4 & 1 & 5 & 5 & 8 \\
$B_{s} \rightarrow \bar{K}^{* 0} \rho^{0}$ & 10 & 9 & 10 & 34 & 3 & 6 & 21 & 12 & 2 & 1 & 5 & 9 & 8 \\
$B_{s} \rightarrow \bar{K}^{* 0} K^{* 0}$ & 14 & 9 & 10 & 32 & 4 & 7 & 25 & 11 & 2 & 1 & 7 & 7 & 8 \\
$B_{s} \rightarrow \phi \rho^{0}$ & 10 & 9 & 9 & 28 & 5 & 0 & 20 & 10 & 2 & 1 & 7 & 10 & 8 \\
$B_{s} \rightarrow \phi K^{* 0}$ & 10 & 8 & 10 & 28 & 6 & 1 & 20 & 10 & 2 & 1 & 9 & 11 & 8 \\
$B_{s} \rightarrow \phi \phi$ & 10 & 8 & 10 & 28 & 6 & 1 & 20 & 10 & 2 & 1 & 11 & 11 & 8 \\
\hline$V X D 3$ & & & & & & & & & & & & & \\
\hline$B_{s} \rightarrow \rho^{0} \rho^{0}$ & 8 & 12 & 14 & 25 & 4 & 3 & 14 & 14 & 5 & 1 & 5 & 5 & 8 \\
$B_{s} \rightarrow \bar{K}^{* 0} \rho^{0}$ & 10 & 9 & 8 & 28 & 3 & 6 & 23 & 12 & 2 & 0 & 5 & 9 & 8 \\
$B_{s} \rightarrow \bar{K}^{* 0} K^{* 0}$ & 10 & 9 & 9 & 29 & 3 & 6 & 22 & 10 & 2 & 1 & 7 & 7 & 8 \\
$B_{s} \rightarrow \phi \rho^{0}$ & 11 & 8 & 9 & 24 & 6 & 0 & 18 & 10 & 2 & 1 & 7 & 10 & 9 \\
$B_{s} \rightarrow \phi K^{* 0}$ & 10 & 8 & 10 & 28 & 6 & 1 & 20 & 10 & 2 & 1 & 9 & 11 & 8 \\
$B_{s} \rightarrow \phi \phi$ & 10 & 8 & 10 & 28 & 6 & 1 & 20 & 10 & 2 & 1 & 11 & 11 & 8 \\
\hline \hline
\end{tabular}

Table 5.6: A list of the parameters that are used in the discriminator functions for $B_{s} \rightarrow$ $V V$

$\mathcal{M}$ is the mass measure described earlier. Here, $m_{1}=5 \mathrm{GeV}, \tilde{I}_{0}=0.1 \mathrm{~cm}^{-1}$ and $I_{0}$ $=0.01 \mathrm{~cm}$, and the vector mass widths are set to $\delta m_{\rho}=0.08 \mathrm{GeV}, \delta m_{K^{*} *}=0.06$ $\mathrm{GeV}$ and $\delta m_{\phi}=0.007 \mathrm{GeV}$ and the vertex mass width is set to $\delta m_{b}=0.1 \mathrm{Gev}$. Tables $5.5\left(B^{0}\right)$ and $5.6\left(B_{s}\right)$ show the remaining parameters,

In Figure 5.11 the distribition of the discriminator values for the $B^{0} \rightarrow \pi^{+} \pi^{-}$ mode is displayed. The background events are shown as bar chart histograms and signal events are shown with solid curve histograms. In Figures B.1 to B.8 a comprehensive list of the discriminator values are plotted for all modes. Only events that pass the direct cuts are included, and a significant separation can be seen. The area 


\section{The Rare Decay Event Selection Algorithm}
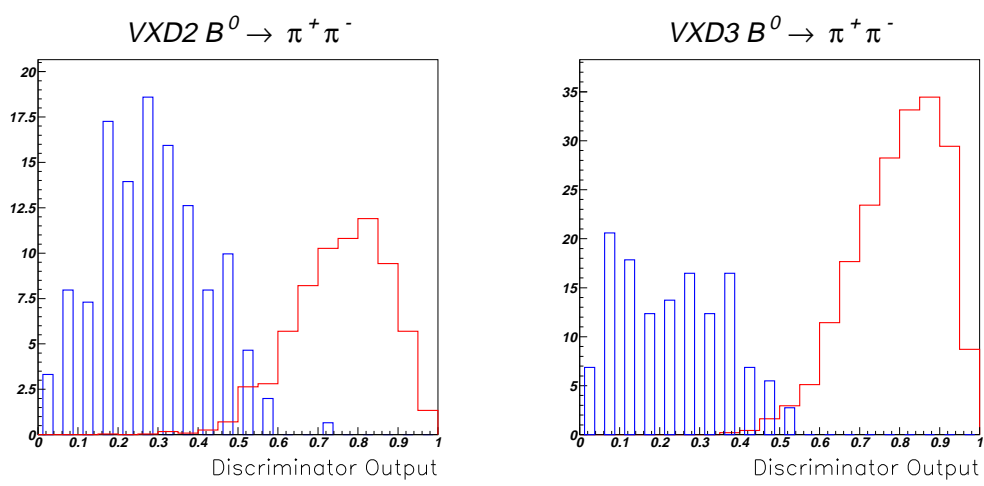

Figure 5.11: Shown are the discriminator values for the $B \rightarrow \pi^{+} \pi^{-}$mode. The bar chart histograms are the background $\mathrm{MC}$ events and the solid histograms are the signal MC events. The area of the background events corresponds to the number of background events to be seen in the data. The area of the signal events is the number of signal events that will be seen if there is $10(25)$ events totally produced at the SLD during the VXD2 (VXD3) period.

of the background distributions corresponds to the number of background events expected in the data. The area of the signal distributions corresponds to the number of events that would be found if there is 10 events (VXD2) or 25 events (VXD3) totally produced in the data. This would correspond to a branching ratio of $3 \cdot 10^{-4}$ for each of the $B^{0}$ and $B^{+}$rare decay modes and a branching ratio of $1 \cdot 10^{-3}$ for each of the $B_{s}$ modes. 


\subsection{Optimal Point of Analysis}
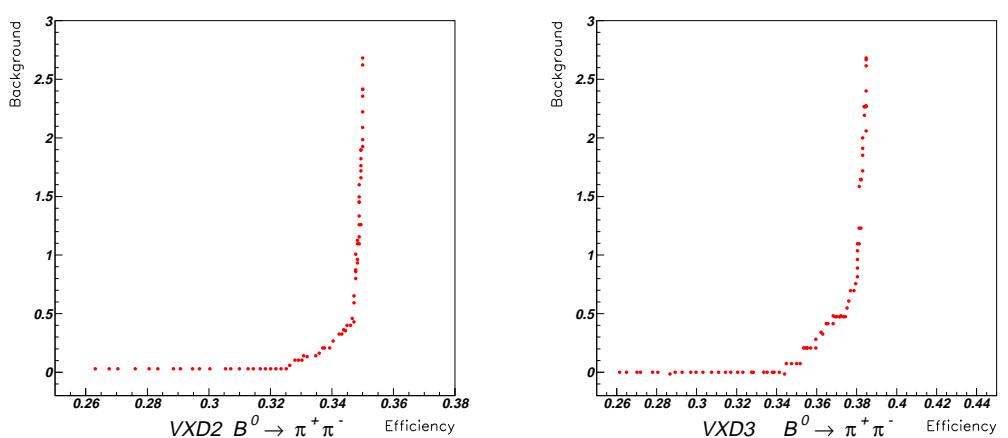

Figure 5.12: Shown are the background versus the efficiency for the $B \rightarrow \pi^{+} \pi^{-}$mode.

\subsection{Optimal Point of Analysis}

At this stage the tuning of the event selection is essentially fixed and what now follows is a procedure for finding the most useful cut on the various discriminator functions. By scanning the discriminator function values for each mode separately, a map of the efficiency versus the expected background can be found. Figure 5.12 shows the background plotted on the vertical axis versus the expected efficiency on the horizontal axis exemplified by $B \rightarrow \pi^{+} \pi^{-}$. Figures B.9 to B.16 includes a comprehensive list. Of interest is the optimal point of analysis for the entire SLD run (combined VXD2 and VXD3), and since this does not necessarily correspond to the most optimal point of analysis for the two VXD subsets independently, the combined efficiency values and the combined background values are calculated. While the 


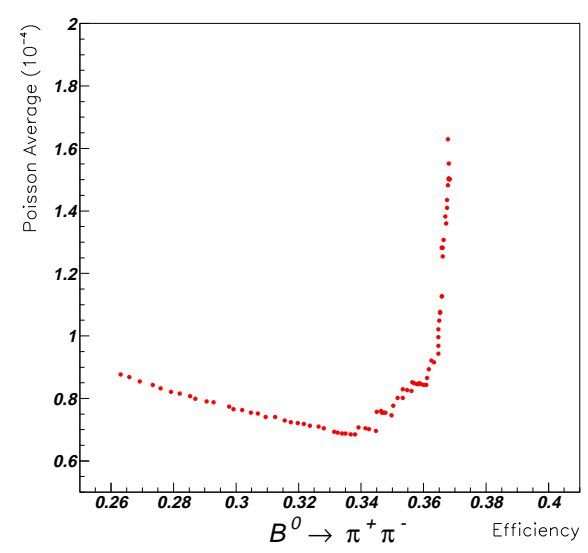

Figure 5.13: Shown are the combined Poisson average versus the efficiency for the $B^{0} \rightarrow$ $\pi^{+} \pi^{-}$mode.

combined background $u$ is simply the sum of the backgrounds from the two periods, the combined efficiency $\varepsilon$ is related to the individual efficiencies by

$$
\varepsilon=\frac{1}{1+r}\left\{\varepsilon_{V X D 2}+r \varepsilon_{V X D 3}\right\}
$$

where $r$ is the ratio of the VXD3 data sample to the VXD2 data sample, $r=2.55$. According to the theoretical branching ratios it is unlikely that any signal events will show up in the data, and the average upper limit (90\% CL) on the branching ratio can then be estimated from the MC efficiencies and backgrounds in the case of zero signal events. This limit, the average Poisson upper limit $\mathcal{P}$, is given by

$$
\mathcal{P}=\sum_{i=0}^{\infty} P(u, i) B r_{i}(\varepsilon)
$$




\subsection{Optimal Point of Analysis}

\begin{tabular}{l||cc|cc|ccc||}
\multicolumn{1}{l||}{} & \multicolumn{2}{c|}{ VXD2 } & \multicolumn{2}{c|}{ VXD3 } & \multicolumn{3}{c||}{ Combined } \\
\hline Mode & $\epsilon$ & cut & $\epsilon$ & cut & $\epsilon$ & bckg. & $\langle B r\rangle \times 10^{4}$ \\
\hline$B^{0} \rightarrow \pi^{+} \pi^{-}$ & 0.325 & 0.575 & 0.344 & 0.550 & 0.338 & 0.033 & 0.680 \\
$B^{0} \rightarrow K^{-} \pi^{+}$ & 0.319 & 0.565 & 0.356 & 0.565 & 0.345 & 0.135 & 0.712 \\
$B^{0} \rightarrow K^{+} K^{-}$ & 0.298 & 0.610 & 0.358 & 0.630 & 0.341 & 0.135 & 0.721 \\
$B_{s} \rightarrow \pi^{+} \pi^{-}$ & 0.316 & 0.615 & 0.403 & 0.565 & 0.379 & 0.033 & 2.298 \\
$B_{s} \rightarrow K^{+} \pi^{-}$ & 0.317 & 0.605 & 0.343 & 0.605 & 0.335 & 0.102 & 2.714 \\
$B_{s} \rightarrow K^{+} K^{-}$ & 0.297 & 0.525 & 0.316 & 0.565 & 0.311 & 0.204 & 3.121 \\
\hline$B^{+} \rightarrow \rho^{0} \pi^{+}$ & 0.240 & 0.600 & 0.285 & 0.645 & 0.272 & 0.339 & 1.017 \\
$B^{+} \rightarrow \rho^{0} K^{+}$ & 0.216 & 0.590 & 0.283 & 0.685 & 0.264 & 0.407 & 1.088 \\
$B^{+} \rightarrow K^{* 0} \pi^{+}$ & 0.227 & 0.645 & 0.308 & 0.635 & 0.285 & 0.170 & 1.322 \\
$B^{+} \rightarrow K^{* 0} K^{+}$ & 0.192 & 0.645 & 0.270 & 0.635 & 0.248 & 0.170 & 1.518 \\
$B^{+} \rightarrow \phi \pi^{+}$ & 0.280 & 0.615 & 0.309 & 0.600 & 0.301 & 0.069 & 1.596 \\
$B^{+} \rightarrow \phi K^{+}$ & 0.271 & 0.580 & 0.340 & 0.560 & 0.321 & 0.137 & 1.564 \\
\hline
\end{tabular}

Table 5.7: The preferred cuts and the corresponding efficiencies and backgrounds for the $B \rightarrow P P$ and $B \rightarrow P V$ modes. The optimal point measure $\langle B r\rangle$, as described in the text, is also listed.

where $P(u, i)$ is the Poisson probability for finding $i$ events given a background of $u$, and $\operatorname{Br}_{i}(\varepsilon)$ is the $90 \%$ CL on the upper limit for the branching ratio if $i$ events are found. Note that $\operatorname{Br}(\varepsilon) \propto 1 / \varepsilon$. Thus, this Poisson average, or expected average 90\% CL upper limit for the branching ratio in the limit of 0 signal (but taking background into account), is a measure of how a particular efficiency and background setting (corresponding to a unique cut on the discriminator function) will influence the found upper limit if no signal events are found in data. The optimal point is then chosen as the minimal solution to this average Poisson upper limit.

Figure 5.13 shows the combined Poisson average for several cuts on the VXD2 
160

The Rare Decay Event Selection Algorithm

\begin{tabular}{l||cc|cc|ccc||}
\multicolumn{1}{c||}{} & \multicolumn{2}{c|}{ VXD2 } & \multicolumn{2}{c|}{ VXD3 } & \multicolumn{3}{c||}{ Combined } \\
\hline Mode & $\epsilon$ & cut & $\epsilon$ & cut & $\epsilon$ & bckg. & $\langle B r\rangle \times 10^{4}$ \\
\hline$B^{0} \rightarrow \rho^{0} \rho^{0}$ & 0.245 & 0.565 & 0.279 & 0.560 & 0.270 & 0.306 & 1.008 \\
$B^{0} \rightarrow \bar{K}^{* 0} \rho^{0}$ & 0.194 & 0.600 & 0.284 & 0.480 & 0.259 & 0.478 & 1.724 \\
$B^{0} \rightarrow \bar{K}^{* 0} K^{* 0}$ & 0.245 & 0.600 & 0.338 & 0.475 & 0.312 & 0.272 & 1.925 \\
$B^{0} \rightarrow \phi \rho^{0}$ & 0.256 & 0.470 & 0.324 & 0.415 & 0.305 & 0.137 & 1.645 \\
$B^{0} \rightarrow \phi \bar{K}^{* 0}$ & 0.253 & 0.560 & 0.322 & 0.440 & 0.302 & 0.137 & 2.488 \\
$B^{0} \rightarrow \phi \phi$ & 0.258 & 0.135 & 0.316 & 0.255 & 0.300 & 0.000 & 3.116 \\
\hline$B_{s} \rightarrow \rho^{0} \rho^{0}$ & 0.239 & 0.555 & 0.301 & 0.555 & 0.284 & 0.272 & 3.553 \\
$B_{s} \rightarrow \bar{K}^{* 0} \rho^{0}$ & 0.228 & 0.590 & 0.299 & 0.555 & 0.279 & 0.343 & 5.640 \\
$B_{s} \rightarrow \bar{K}^{* 0} K^{* 0}$ & 0.208 & 0.620 & 0.298 & 0.545 & 0.272 & 0.170 & 7.361 \\
$B_{s} \rightarrow \phi \rho^{0}$ & 0.236 & 0.505 & 0.321 & 0.465 & 0.297 & 0.069 & 6.112 \\
$B_{s} \rightarrow \phi K^{* 0}$ & 0.254 & 0.450 & 0.280 & 0.465 & 0.272 & 0.137 & 10.437 \\
$B_{s} \rightarrow \phi \phi$ & 0.254 & 0.205 & 0.340 & 0.270 & 0.316 & 0.000 & 11.182 \\
\hline
\end{tabular}

Table 5.8: The preferred cuts and the corresponding efficiencies and backgrounds for the $B \rightarrow V V$ modes. The optimal point measure $\langle B r\rangle$, as described in the text, is also listed.

and the VXD3 discriminator functions for the $B \rightarrow \pi^{+} \pi^{-}$mode. The combination of VXD2 and VXD3 does give a 2-d space, but to simplify the visual presentation for the combined result a selected set of favorable cut points are choosen to be included in the plot.

Figures B.17 to B.20 show a comprehensive list. In Tables 5.7 and 5.8 the most preferred cuts on the discriminator functions are listed with the efficiency for the VXD2 and the VXD3 run periods, and for the combined event sample, the estimated background and efficiency are listed together with the Poisson average. The actual limit will improve if no events are found in the data and vise versa, it will increase 


\subsection{Optimal Point of Analysis}

\begin{tabular}{|lc|lc|} 
Mode & Background & Mode & Background \\
\hline$B^{0} \rightarrow \pi^{+} \pi^{-}$ & None & $B^{0} \rightarrow \rho^{0} \rho^{0}$ & $I^{(0.81)}$ \\
$B^{0} \rightarrow K^{-} \pi^{+}$ & $A^{(0.74)}$ & $B^{0} \rightarrow \bar{K}^{* 0} \rho^{0}$ & $I^{(0.86)}, J^{(0.70)}$ \\
$B^{0} \rightarrow K^{+} K^{-}$ & $A^{(0.77)}$ & $B^{0} \rightarrow \bar{K}^{* 0} K^{* 0}$ & $I^{(0.86)}, K^{(0.62)}, L^{(0.63)}$ \\
$B_{s} \rightarrow \pi^{+} \pi^{-}$ & None & $B^{0} \rightarrow \phi \rho^{0}$ & $M^{(0.63)}, N^{(0.67)}$ \\
$B_{s} \rightarrow K^{+} \pi^{-}$ & $A^{(0.69)}$ & $B^{0} \rightarrow \phi \bar{K}^{* 0}$ & $M^{(0.66)}, N^{(0.75)}$ \\
$B_{s} \rightarrow K^{+} K^{-}$ & $A^{(0.67)}, B^{(0.60)}$ & $B^{0} \rightarrow \phi \phi$ & None \\
\hline$B^{+} \rightarrow \rho^{0} \pi^{+}$ & $A^{(0.74)}, C^{(0.77)}, D^{(0.82)}$ & $B_{s} \rightarrow \rho^{0} \rho^{0}$ & None \\
$B^{+} \rightarrow \rho^{0} K^{+}$ & $C^{(0.74)}, D^{(0.81)}, E^{(0.70)}, F^{(0.76)}$ & $B_{s} \rightarrow \bar{K}^{* 0} \rho^{0}$ & $I^{(0.82)}, J^{(0.68)}$ \\
$B^{+} \rightarrow K^{* 0} \pi^{+}$ & $F^{(0.69)}$ & $B_{s} \rightarrow \bar{K}^{* 0} K^{* 0}$ & $I^{(0.87)}$ \\
$B^{+} \rightarrow K^{* 0} K^{+}$ & $F^{(0.69)}$ & $B_{s} \rightarrow \phi \rho^{0}$ & $N^{(0.62)}$ \\
$B^{+} \rightarrow \phi \pi^{+}$ & $G^{(0.78)}$ & $B_{s} \rightarrow \phi K^{* 0}$ & $M^{(0.61)}, N^{(0.68)}$ \\
$B^{+} \rightarrow \phi K^{+}$ & $G^{(0.78)}, H^{(0.63)}$ & $B_{s} \rightarrow \phi \phi$ & None \\
\hline
\end{tabular}

Table 5.9: From a total of $1.0 \mathrm{M} b \bar{b}$ MC events a total of 14 events pass the discriminator cuts. Most of these events show up in several modes.

if events are seen. It can be seen that the efficiencies are in the range $25 \%$ to $37 \%$, where the $B_{s} \rightarrow \pi^{+} \pi^{-}$mode has a particularly high efficiency. With only two pion tracks, it is hard for two track combinations in the background events to pass the $B_{s}$ mass cut, and the resulting event selection allows for somewhat loose cuts without any increase in the expected background.

The background estimates are based upon a MC $b \bar{b}$ background sample of $1.1 \mathrm{M}$ and 1.4 M events for VXD2 and VXD3 respectively. The expected levels are in the range from 0.0 to 0.5 and while this is quite low and satisfactory, it can be useful to study the few background events that do pass the cuts to see if further improvements can be made. A $1.0 \mathrm{M} b \bar{b}$ MC background sample was studied for the VXD3 period 


\section{The Rare Decay Event Selection Algorithm}

\begin{tabular}{|c|c|c|}
\hline Event & Tagged tracks & \\
\hline$A$ & $B^{-} \rightarrow \underline{\pi}^{+} \bar{D}^{0} \rightarrow \underline{\pi}^{+}\left(K^{+} \rho^{-}\right) \rightarrow \underline{\pi}^{+} K^{+}\left(\underline{\pi}^{-} \pi^{0}\right)$ & Two tracks \\
\hline$A$ & $B^{-} \rightarrow \underline{\pi}^{+} \bar{D}^{0} \rightarrow \underline{\pi}^{+}\left(\underline{K}^{+} \rho^{-}\right) \rightarrow \underline{\pi}^{+} \underline{K}^{+}\left(\underline{\pi}^{-} \pi^{0}\right)$ & Three tracks \\
\hline$B$ & $B^{0} \rightarrow \bar{\nu}_{\mu} \mu^{-} D^{* 0} ; f \rightarrow \underline{\pi}^{+}$ & \\
\hline$C$ & $B^{+} \rightarrow \underline{K}^{-} J / \psi \rightarrow \underline{K}^{-}\left(\underline{\mu}^{+} \underline{\mu}^{-}\right)$ & \\
\hline$D$ & $B_{s} \rightarrow \underline{\pi}^{-} D_{s} \rightarrow \underline{\pi}^{-}\left(\underline{\pi}^{+} \bar{f}\right) \rightarrow \underline{\pi}^{-} \underline{\pi}^{+}\left(\underline{\pi}^{-} \pi^{+}\right)$ & \\
\hline$E$ & $B^{0} \rightarrow \underline{\pi}^{+} D^{-} \rightarrow \underline{\pi}^{+}\left(\underline{K}^{+} \underline{\pi}^{-} \pi^{-}\right)$ & \\
\hline$F$ & $B^{-} \rightarrow \underline{\pi}^{-} \bar{D}^{* 0} \rightarrow \underline{\pi}^{-}\left(\pi^{0} D^{0}\right) \rightarrow \underline{\pi}^{-} \pi^{0}\left(\underline{K}^{-} \underline{\pi}^{+}\right)$ & \\
\hline G & $B^{-} \rightarrow \underline{\pi}^{-} D^{0} \rightarrow \underline{\pi}^{-}\left(\pi^{0} \phi\right) \rightarrow \underline{\pi}^{-} \pi^{0}\left(\underline{K}^{+} \underline{K}^{-}\right)$ & \\
\hline$H$ & $\bar{B}_{s} \rightarrow \underline{\pi}^{+} D_{s} \rightarrow \underline{\pi}^{+}\left(\pi^{-} \phi\right) \rightarrow \underline{\pi}^{+} \pi^{-}\left(\underline{K}^{+} \underline{K}^{-}\right)$ & \\
\hline$I$ & $\bar{B}^{0} \rightarrow \underline{K}^{+} \underline{\pi}^{-} J / \psi \rightarrow \underline{K}^{+} \underline{\pi}^{-}\left(\underline{e}^{+} \underline{e}^{-}\right)$ & \\
\hline$J$ & $\bar{B}^{0} \rightarrow \underline{K}^{+} \underline{\pi}^{-} J / \psi \rightarrow \underline{K}^{+} \underline{\pi}^{-}\left(\underline{\mu}^{+} \underline{\mu}^{-}\right)$ & \\
\hline$K$ & $\bar{\Lambda}_{c} \rightarrow \underline{P}^{+} \underline{K}^{-} \pi^{+} \pi^{0}$ & \\
\hline$L$ & $B_{s} \rightarrow \underline{\pi}^{-} \rho^{0} D_{s} \rightarrow \underline{\pi}^{-}\left(\underline{\pi}^{+} \pi^{-}\right)\left(\underline{\pi}^{+} f\right) \rightarrow \underline{\pi}^{-} \underline{\pi}^{+} \pi^{-} \underline{\pi}^{+}\left(\underline{\pi}^{-} \pi^{+}\right)$ & \\
\hline$M$ & $a_{1}^{+} \rightarrow \underline{\pi}^{-} \rho^{0} \rightarrow \underline{\pi}^{-}\left(\underline{\pi}^{+} \underline{\pi}^{-}\right): \quad D_{s} \rightarrow \underline{\pi}^{+} K^{0}$ & \\
\hline$N$ & $B^{+} \rightarrow \underline{K}^{+} f \rightarrow \underline{K}^{+}\left(\underline{\pi}^{-} \pi^{+} J / \psi\right) \rightarrow \underline{K}^{+} \underline{\pi}^{-} \pi^{+}\left(\underline{e}^{+} \underline{e}^{-}\right)$ & \\
\hline
\end{tabular}

Table 5.10: Detailed list of the background events are shown. The tagged particles are underlined. $f$ is an unspecified fragmentation string [64].

and Table 5.9 shows the MC events $(A, B, C, D, E, F, G, H, I, J, K, L, M$ and $N$ ) that pass the cuts with the corresponding discriminator values indicated in the brackets. The MC background events $(A, \ldots)$ are shown in Table 5.10 with the tagged particles being underlined. A total of 14 distinct events pass the discriminator cuts and most of these show up in several modes. Section 5.6 will discuss the possible use of particle ID, its impact on the efficiency and purity, and a conclusion of whether particle ID will be used or not is made. 


\subsection{Cross Check Mode}

\subsection{Cross Check Mode}

In the search for rare $B$ decays there are for obvious reasons a lack of data to use in comparison with the MC used in the study. In order to cross check the efficiency calculations and generally the integrity of the methods, the $D^{+} \rightarrow K^{-} \pi^{+} \pi^{+}$mode is studied. The idea is to reinvent the reconstruction of this benchmark mode using similar techniques as is used for the rare $B$ decay analysis. For this, the entire 1996-98 data sample and a MC sample 1.76 times as large is investigated.

Similar to rare $B$ decay analysis, three track combinations are formed and a kaon mass is assigned to the track with the opposite charge compared to the vertex charge. A set of direct cuts is applied. The reconstructed vertex mass must be within 1.72.1 GeV, the vertex significance must be larger than 1.5 and the normalized track impact parameter must be at least 0.08 . The vertex probability must be at least 0.1 $\%$ and at least one of the tracks must have a momentum larger than $1 \mathrm{GeV}$.

The discriminator function takes the following form

$$
\mathcal{F}_{D}=-9 e^{-\frac{S}{4.0}}-3 e^{-p_{s}}-3 e^{-\frac{p_{l}}{3}}-e^{-\frac{P}{0.1}}-2 e^{-\frac{B}{2}}-5 e^{-\frac{X}{0.4}}+4 e^{-\frac{I}{0.002}}-4 e^{\frac{I}{0.04}}
$$

where two parameters are used in addition to the ones for the rare $B$ decay study. They are the smallest $p_{s}$ and largest $p_{l}$ track momentum in a vertex.

Figure 5.14 shows the mass distributions of the MC and the data events. The 


\section{The Rare Decay Event Selection Algorithm}
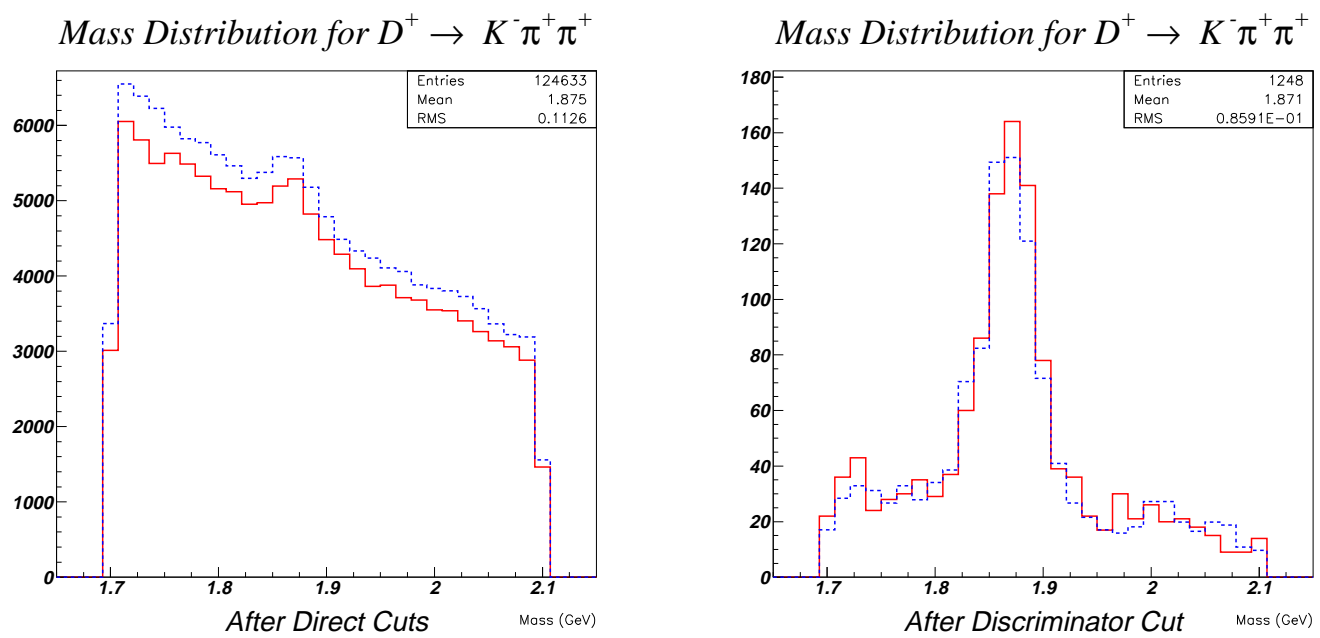

Figure 5.14: Shown are the benchmark mode $D^{+} \rightarrow K^{-} \pi^{+} \pi^{+}$mass distributions after the direct cuts and whit a added cut on the discriminator values. Data are shown in solid histograms while MC are plotted with dashed histograms. The statistics are for the data.

left plot shows this distribution after the direct cuts are applied and the right plot shows the same distribution when an additional cut is made on the distribution function. The data is shown as a solid histogram while the MC is shown as dashed histogram. Before the discriminator cut a small excess in the MC can be seen. After the discriminator cut the data and $\mathrm{MC}$ agree within $3 \%$ and is well within the experimental understanding of the branching ratio for $D^{+} \rightarrow K^{-} \pi^{+} \pi^{+}$and the $D$ meson production rate.

This shows that the MC modeling of the $D^{+} \rightarrow K^{-} \pi^{+} \pi^{+}$mode is well understood and the event selection method seems reliable. The corrections explained in Chapter 


\subsection{The Case Against Using Particle ID}

4 are applied to the MC prior to this study.

\subsection{The Case Against Using Particle ID}

In Table 5.10 a set of background events are listed with information of the particular process and the tagged tracks. The 14 events show up in several modes with a total count of 33 occurrences. In the case of a $100 \%$ positively identification efficiency most of the background events can be removed and only 4 distinct background events will remain with a total of 5 occurrences $^{3}$. As a preliminary study the benchmark mode $D^{+} \rightarrow K^{-} \pi^{+} \pi^{+}$will be investigated with respect to pion, kaon and proton identification in the CRID. Particle ID is done with the SLD 'CKID' package [69].

The $D^{+} \rightarrow K^{-} \pi^{+} \pi^{+}$event sample contains approximately $50 \%$ true events and $50 \%$ background events. The first step will be to compare data and MC for consistency.

- If CKID returns a track as a positively defined proton the corresponding vertex will be rejected. The remaining fraction in the data and the $\mathrm{MC}$ are then $95.9 \%$ and $95.8 \%$ respectively.

\footnotetext{
${ }^{3}$ The remaining background events are $D$ in mode $B^{+} \rightarrow \rho^{0} \pi^{+}, E$ and $F$ in mode $B^{+} \rightarrow \rho^{0} K^{+}$, $F$ in mode $B^{+} \rightarrow K^{* 0} \pi^{+}$and $G$ in mode $B^{+} \rightarrow \phi \pi^{+}$.
} 


\section{The Rare Decay Event Selection Algorithm}

- If a track is assigned a kaon mass and the CKID returns the track as a positively defined pion the event will be rejected. The remaining fraction in the data and the $\mathrm{MC}$ are then $88.9 \%$ and $90.8 \%$ respectively, and the combined effect of the pion rejection and proton rejection gives a remaining fraction of $84.8 \%$ (data) and $86.9 \%(\mathrm{MC})$.

- If a track is assigned a pion mass and the CKID returns the track as a positively defined kaon the event will be rejected. The remaining fraction in the data and the $\mathrm{MC}$ are then $91.2 \%$ and $92.9 \%$ respectively. The combined effect of the kaon rejection, pion rejection and proton rejection gives a remaining fraction of $77.0 \%$ (data) and $80.0 \%$ (MC).

These results indicates that the MC models the CRID particle ID fairly well (within $4 \%$ ) and the MC event sample will therefore be used for more detailed studies. Table 5.11 shows the tagging purity and tagging efficiency for pion, kaons and protons as found from the MC benchmark mode. CorrectID is the fraction when a particular particle is correctly identified, and MisID is the fraction when a particular particle is positively identified, but the identification is wrong. Based upon these numbers it is clear that an event selection scheme that aims for a reconstruction of only positively identified tracks will drastically lower the efficiency. For the $B^{0} \rightarrow \rho^{0} \rho^{0}$ mode, 


\subsection{The Case Against Using Particle ID}

\begin{tabular}{ccc} 
& CorrectID & MisID \\
\hline$\pi$ & $32 \%$ & $1.6 \%$ \\
$\mathrm{~K}$ & $23 \%$ & $6.9 \%$ \\
$\mathrm{P}$ & $22 \%$ & $23 \%$
\end{tabular}

Table 5.11: Shown are the efficiencies (CorrectID) and purity (MisID) for the pion, kaon and proton tracks.

for example, with four pions in the final state the efficiency would be reduced to approximately $1 \%\left(=0.32^{4}\right)$ of the already reduced sample making up the candidate vertices. However, another and feasible approach of particle ID that will be investigated is the possible use of particle veto. Again, for the $B^{0} \rightarrow \rho^{0} \rho^{0}$ mode, the veto idea will be to throw away events where any track is positively identified as a proton or a kaon. According to the above Table, around $6.4 \%(4.6 \%)$ of the true events will then be lost, but the hope is that the background rejection will be significantly stronger such that the overall analysis will benefit.

The particle veto algorithm is as follows. For all the modes, events are thrown away if any of the tracks is identified as a proton, and for modes that only involve one type of particle, kaon or pion rejection is rather straightforward to implement, as explained above for kaon rejection in the $B^{0} \rightarrow \rho^{0} \rho^{0}$ mode. For other modes with a combination of kaons and pions in the final state some complications naturally occur due to the track assignment procedure described in Chapter 5. For example, for the two tracks $i, j$ in the $B^{0} \rightarrow K^{-} \pi^{+}$mode, the reconstructed vertex mass $m_{B}$ 
The Rare Decay Event Selection Algorithm

\begin{tabular}{l|cc|cc|c} 
& VXD2 & & VXD3 & & $\delta\langle B r\rangle \times 10^{4}$ \\
& bckg. & signal & bckg. & signal & \\
\hline \hline$B^{0} \rightarrow \pi^{+} \pi^{-}$ & 0.910 & 0.959 & 0.819 & 0.976 & 0.016 \\
\hline$B^{0} \rightarrow K^{-} \pi^{+}$ & 0.957 & 0.966 & 0.817 & 0.963 & 0.021 \\
\hline$B^{0} \rightarrow K^{+} K^{-}$ & 0.612 & 0.963 & 0.458 & 0.938 & 0.010 \\
\hline$B_{s} \rightarrow \pi^{+} \pi^{-}$ & 0.915 & 0.961 & 0.786 & 0.971 & 0.069 \\
\hline$B_{s} \rightarrow K^{+} \pi^{-}$ & 0.969 & 0.973 & 0.815 & 0.957 & 0.073 \\
\hline$B_{s} \rightarrow K^{+} K^{-}$ & 0.609 & 0.969 & 0.482 & 0.942 & -0.017 \\
\hline \hline$B^{+} \rightarrow \rho^{0} \pi^{+}$ & 0.709 & 0.934 & 0.748 & 0.954 & 0.006 \\
\hline$B^{+} \rightarrow \rho^{0} K^{+}$ & 0.932 & 0.969 & 0.868 & 0.957 & 0.016 \\
\hline$B^{+} \rightarrow K^{* 0} \pi^{+}$ & 0.949 & 0.948 & 0.874 & 0.947 & 0.053 \\
\hline$B^{+} \rightarrow K^{* 0} K^{+}$ & 0.857 & 0.957 & 0.764 & 0.928 & 0.066 \\
\hline$B^{+} \rightarrow \phi \pi^{+}$ & 0.812 & 0.960 & 0.801 & 0.916 & 0.100 \\
\hline$B^{+} \rightarrow \phi K^{+}$ & 0.575 & 0.930 & 0.519 & 0.893 & 0.088 \\
\hline \hline
\end{tabular}

Table 5.12: The effect of using the CRID particle ID in a veto mode for $B \rightarrow P P$ or $P V$. Shown are the remaining fraction of background events and signal events after this veto. The change in the average upper limit $(90 \% \mathrm{CL})$ is shown and there is improvement to be seen in only the $B_{s} \rightarrow K^{+} K^{-}$mode.

is given by

$$
m_{B}^{2}=m_{i}^{2}+m_{j}^{2}+2 \sqrt{p_{i}^{2}+m_{i}^{2}} \sqrt{p_{j}^{2}+m_{j}^{2}}-2 \underline{p}_{i} \cdot \underline{p}_{j}
$$

where $\{i, j\}=\{\pi, K\}$ or $\{i, j\}=\{K, \pi\}$. If the total momentum of the individual tracks are equal or close to equal the track mass assignment is rather random since both possible combinations can give close to identical vertex masses. Then, for such modes the rejection algorithm will not be based upon a track to track identification, but rather, an event is rejected if a particular particle is found more often than what is allowed. For $B^{0} \rightarrow K^{-} \pi^{+}$,for example, this implies that the event can be rejected 
169

\subsection{The Case Against Using Particle ID}

\begin{tabular}{l|ll|ll|c} 
& VXD2 & & VXD3 & & $\delta\langle B r\rangle \times 10^{4}$ \\
& bckg. & signal & bckg. & signal & \\
\hline \hline$B^{0} \rightarrow \rho^{0} \rho^{0}$ & 0.813 & 0.932 & 0.720 & 0.939 & 0.020 \\
\hline$B^{0} \rightarrow \bar{K}^{* 0} \rho^{0}$ & 0.958 & 0.947 & 0.883 & 0.941 & 0.058 \\
\hline$B^{0} \rightarrow \bar{K}^{* 0} K^{* 0}$ & 0.908 & 0.963 & 0.857 & 0.914 & 0.124 \\
\hline$B^{0} \rightarrow \phi \rho^{0}$ & 0.925 & 0.955 & 0.796 & 0.915 & 0.095 \\
\hline$B^{0} \rightarrow \phi K^{* 0}$ & 0.709 & 0.928 & 0.621 & 0.889 & 0.189 \\
\hline$B^{0} \rightarrow \phi \phi$ & - & 0.922 & 0.333 & 0.843 & 0.513 \\
\hline \hline$B_{s} \rightarrow \rho^{0} \rho^{0}$ & 0.801 & 0.934 & 0.734 & 0.948 & 0.067 \\
\hline$B_{s} \rightarrow \bar{K}^{* 0} \rho^{0}$ & 0.960 & 0.941 & 0.872 & 0.927 & 0.289 \\
\hline$B_{s} \rightarrow \bar{K}^{* 0} K^{* 0}$ & 0.902 & 0.973 & 0.871 & 0.914 & 0.543 \\
\hline$B_{s} \rightarrow \phi \rho^{0}$ & 0.912 & 0.950 & 0.778 & 0.892 & 0.560 \\
\hline$B_{s} \rightarrow \phi K^{* 0}$ & 0.701 & 0.931 & 0.609 & 0.896 & 0.645 \\
\hline$B_{s} \rightarrow \phi \phi$ & - & 0.940 & - & 0.807 & 2.042 \\
\hline \hline
\end{tabular}

Table 5.13: The effect of using the CRID particle ID in a veto mode for $B \rightarrow V V$. Shown are the remaining fraction of background events and signal events after this veto. The change in the average upper limit $(90 \% \mathrm{CL})$ is shown and no improvements are expected by using the CRID.

if the particle ID suggests that both tracks are pions or that both are kaons.

Tables 5.12 and 5.13 show the effect of particle ID veto on vertices that pass the direct cuts. These results are then used to scale the efficiencies and background levels accordingly and the corresponding optimal point of analysis is then re-calculated. Tables 5.12 and 5.13 show the change in the Poisson average compared to the results listed in Tables 5.7 and 5.8. A minus sign indicates that the average upper limit will improve (decrease) compared to the limit expected if no CRID particle ID is used. It can be seen that there is only one mode $\left(B_{s} \rightarrow K^{+} \pi^{-}\right)$where some marginal 


\section{The Rare Decay Event Selection Algorithm}

improvement is expected. All the other modes show a marginal to significant degrading of the expected upper limits. These are the arguments for why the analysis does not use CRID particle ID as a classification method of rare $B$ decays.

A similar study was done to investigate the possible use of muon and electron rejection, using a standard set of SLD routines[70]. Table 5.14 lists the results and it is concluded that the analysis will not in general benefit from the implementation of electron or muon rejection. Again, this study was performed on MC generated events. 


\subsection{The Case Against Using Particle ID}

\begin{tabular}{l|cc|cc|c} 
& VXD2 & & VXD3 & $\delta\langle B r\rangle \times 10^{4}$ \\
& bckg. & signal & bckg. & signal & \\
\hline \hline$B^{0} \rightarrow \pi^{+} \pi^{-}$ & 0.832 & 0.984 & 0.813 & 0.986 & 0.008 \\
\hline$B^{0} \rightarrow K^{-} \pi^{+}$ & 0.816 & 0.984 & 0.855 & 0.982 & 0.003 \\
\hline$B^{0} \rightarrow K^{+} K^{-}$ & 0.817 & 0.988 & 0.832 & 0.985 & -0.001 \\
\hline$B_{s} \rightarrow \pi^{+} \pi^{-}$ & 0.823 & 0.992 & 0.808 & 0.987 & 0.017 \\
\hline$B_{s} \rightarrow K^{+} \pi^{-}$ & 0.836 & 0.986 & 0.829 & 0.984 & 0.008 \\
\hline$B_{s} \rightarrow K^{+} K^{-}$ & 0.848 & 0.989 & 0.825 & 0.989 & -0.020 \\
\hline \hline$B^{+} \rightarrow \rho^{0} \pi^{+}$ & 0.804 & 0.977 & 0.814 & 0.980 & -0.015 \\
\hline$B^{+} \rightarrow \rho^{0} K^{+}$ & 0.804 & 0.985 & 0.802 & 0.981 & 0.008 \\
\hline$B^{+} \rightarrow K^{* 0} \pi^{+}$ & 0.843 & 0.978 & 0.877 & 0.981 & 0.008 \\
\hline$B^{+} \rightarrow K^{* 0} K^{+}$ & 0.843 & 0.979 & 0.877 & 0.982 & 0.008 \\
\hline$B^{+} \rightarrow \phi \pi^{+}$ & 0.885 & 0.977 & 0.866 & 0.982 & 0.021 \\
\hline$B^{+} \rightarrow \phi K^{+}$ & 0.885 & 0.974 & 0.868 & 0.981 & 0.022 \\
\hline \hline$B^{0} \rightarrow \rho^{0} \rho^{0}$ & 0.896 & 0.971 & 0.896 & 0.981 & 0.004 \\
\hline$B^{0} \rightarrow K^{* 0} \rho^{0}$ & 0.906 & 0.971 & 0.917 & 0.976 & 0.006 \\
\hline$B^{0} \rightarrow K^{* 0} K^{* 0}$ & 0.906 & 0.985 & 0.937 & 0.977 & 0.020 \\
\hline$B^{0} \rightarrow \phi \rho^{0}$ & 0.868 & 0.975 & 0.959 & 0.969 & 0.045 \\
\hline$B^{0} \rightarrow \phi \bar{K}^{* 0}$ & 0.892 & 0.994 & 0.986 & 0.960 & 0.079 \\
\hline$B^{0} \rightarrow \phi \phi$ & 1.000 & 0.976 & 1.000 & 0.994 & 0.033 \\
\hline \hline$B_{s} \rightarrow \rho^{0} \rho^{0}$ & 0.896 & 0.983 & 0.886 & 0.979 & 0.017 \\
\hline$B_{s} \rightarrow \bar{K}^{* 0} \rho^{0}$ & 0.907 & 0.989 & 0.918 & 0.974 & 0.066 \\
\hline$B_{s} \rightarrow K^{* 0} K^{* 0}$ & 0.904 & 0.967 & 0.947 & 0.972 & 0.193 \\
\hline$B_{s} \rightarrow \phi \rho^{0}$ & 0.870 & 0.981 & 0.915 & 0.978 & 0.105 \\
\hline$B_{s} \rightarrow \phi K^{* 0}$ & 0.901 & 1.000 & 0.972 & 0.958 & 0.295 \\
\hline$B_{s} \rightarrow \phi \phi$ & 1.000 & 0.985 & 1.000 & 0.980 & 0.206 \\
\hline \hline
\end{tabular}

Table 5.14: Shown are the remaining fraction of background events and signal events after electron and muon veto. The change in the average upper limit (90\% CL) is shown and in general no improvements are expected by the use of the electron and muon ID. A minus sign would indicate an expected improvement. 



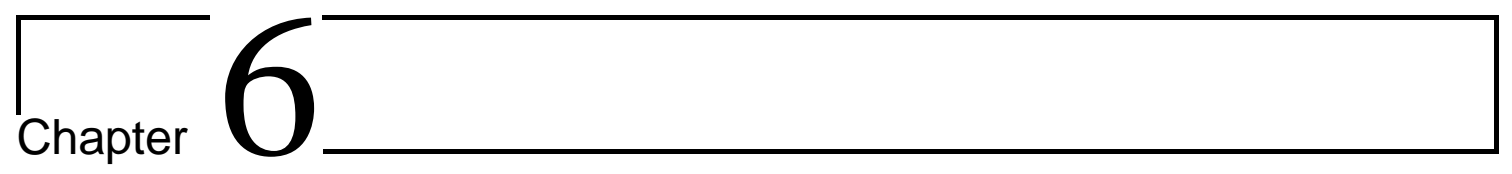

\section{Data and Final Results}

The various versions of the rare decay event selection algorithm are described in Chapter 5. This Chapter will apply these algorithms to the SLD VXD2 and VXD3 data and subsequently turn the outcome into branching ratio results. Section 6.1 will give the events in the data, if any, that pass the cuts and a decision will be made on whether a statistically significant signal is seen or not. Section 6.2 will discuss the statistical methods used and in connection with this the systematic and statistical uncertainties will be estimated. The last section (6.3) will give the final results followed by a summary. 


\section{Data and Final Results}

\begin{tabular}{lcc|lccc} 
Mode & Bckg. & Data & Mode & Bckg. & Data & Event \\
\hline$B^{0} \rightarrow \pi^{+} \pi^{-}$ & 0.03 & 0 & $B^{0} \rightarrow \rho^{0} \rho^{0}$ & 0.31 & 1 & $E_{1}$ \\
$B^{0} \rightarrow K^{-} \pi^{+}$ & 0.14 & 0 & $B^{0} \rightarrow \bar{K}^{* 0} \rho^{0}$ & 0.49 & 2 & $E_{1}, E_{2}$ \\
$B^{0} \rightarrow K^{+} K^{-}$ & 0.14 & 0 & $B^{0} \rightarrow \bar{K}^{* 0} K^{* 0}$ & 0.27 & 3 & $E_{1}, E_{2}, E_{3}$ \\
$B_{s} \rightarrow \pi^{+} \pi^{-}$ & 0.03 & 0 & $B^{0} \rightarrow \phi \rho^{0}$ & 0.14 & 0 & \\
$B_{s} \rightarrow K^{+} \pi^{-}$ & 0.10 & 0 & $B^{0} \rightarrow \phi \bar{K}^{* 0}$ & 0.14 & 1 & $E_{4}$ \\
$B_{s} \rightarrow K^{+} K^{-}$ & 0.20 & 0 & $B^{0} \rightarrow \phi \phi$ & 0.00 & 0 & \\
\hline$B^{+} \rightarrow \rho^{0} \pi^{+}$ & 0.34 & 0 & $B_{s} \rightarrow \rho^{0} \rho^{0}$ & 0.27 & 0 & \\
$B^{+} \rightarrow \rho^{0} K^{+}$ & 0.41 & 0 & $B_{s} \rightarrow \bar{K}^{* 0} \rho^{0}$ & 0.34 & 1 & $E_{1}$ \\
$B^{+} \rightarrow K^{* 0} \pi^{+}$ & 0.17 & 0 & $B_{s} \rightarrow \bar{K}^{* 0} K^{* 0}$ & 0.17 & 2 & $E_{1}, E_{3}$ \\
$B^{+} \rightarrow K^{* 0} K^{+}$ & 0.17 & 0 & $B_{s} \rightarrow \phi \rho^{0}$ & 0.07 & 0 & \\
$B^{+} \rightarrow \phi \pi^{+}$ & 0.07 & 0 & $B_{s} \rightarrow \phi K^{* 0}$ & 0.14 & 0 & \\
$B^{+} \rightarrow \phi K^{+}$ & 0.14 & 0 & $B_{s} \rightarrow \phi \phi$ & 0.00 & 0 &
\end{tabular}

Table 6.1: A total of 4 distinct events show up in the data, $E_{i}$ with $\mathrm{i}=(1,2,3,4)$, with an average multiplicity of 2.5 .

\subsection{Data}

Table 6.1 lists the result of the search which shows no events in the data for the two track $(B \rightarrow P P)$ and the three track $(B \rightarrow P V)$ modes. A few events are seen in the four track modes $(B \rightarrow V V)$ and they are all from the VXD3 (1996-98) run period. Each distinct event $i$ is denoted $E_{i}$ and there are 4 of these events with a total occurrence of 10 times. The expected background level is also listed, and in the limit of no correlation between the various modes, a total of 0.6 distinct background events is expected in $B \rightarrow P P, 1.3$ events in $B \rightarrow P V$ and 2.3 events in $B \rightarrow V V$. However, due to the overlap as pointed out in Chapter 5 , these numbers will be reduced somewhat, and if the amount of correlation in Table 5.10 is a correct 


\subsection{Data}

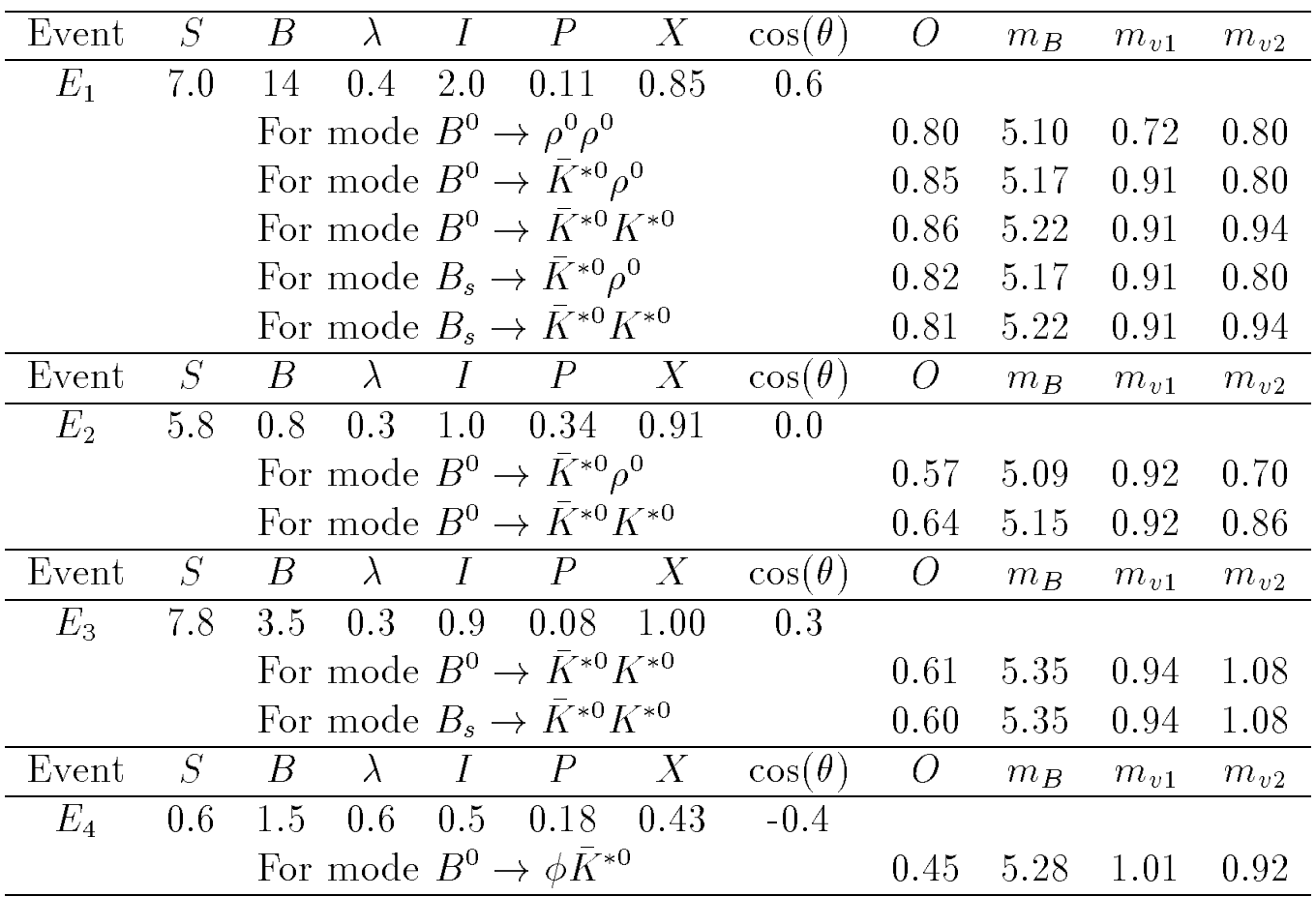

Table 6.2: Shown are the reconstructed parameters for the data events.

measure, the number of distinct background events is reduced to $0.2,0.8$ and 0.9 for $B \rightarrow P P, B \rightarrow P V$ and $B \rightarrow V V$ respectively, giving a total of 1.9 different events. In view of this, the overall number of events (4) is not tremendously off from what is expected from background, however, the four events being located to the $B \rightarrow V V$ modes only is somewhat high compared to the expected 0.9 for these modes.

Table 6.2 lists the reconstructed parameters associated with each event. The notation is the same as in Chapter $5 . S$ is the vertex significance, $B$ is the smallest 


\section{Data and Final Results}

normalized track impact parameter, $\lambda$ is the angular track spread, $I$ is the vertex impact parameter in units of $10^{-3} \mathrm{~cm}, P$ is the vertex probability, $X$ is the fragmentation parameter, $m_{B}$ is the vertex mass and $m_{v i}$ are the vector masses, with $m_{v 1}$ being associated with the heaviest vector meson when relevant, all in units of $\mathrm{GeV}$. The polar angle of the $B$ flight direction $\theta$ is also shown together with the discriminator function output $O$. All events are well within the detector acceptance. Event $E_{4}$ does barely pass the significance cut at 0.6 and the discriminator cut at 0.445, while the other events do comfortably pass the event selection. The statistical significance of these events can be quantified by the accumulated Poisson probability for finding a number of events equal to or larger to what has been found. Mode $B^{0} \rightarrow \bar{K}^{* 0} K^{* 0}$ shows the strongest deviation from the expected background level (0.27) with its three events, and the probability $P$ for finding three or more events is

$$
P=1-\sum_{i=0}^{2} \frac{e^{-0.27}(0.27)^{i}}{i !}=0.27 \%
$$

which is identical with the three sigma limit $(0.27 \%)$ when Gaussian variables are considered. Of course, this calculation relies heavily on the expected background level and the corresponding probability is $8 \%$ if the background level is 0.5 instead of 0.27 . An issue of interest then is to understand how well the background is mod- 

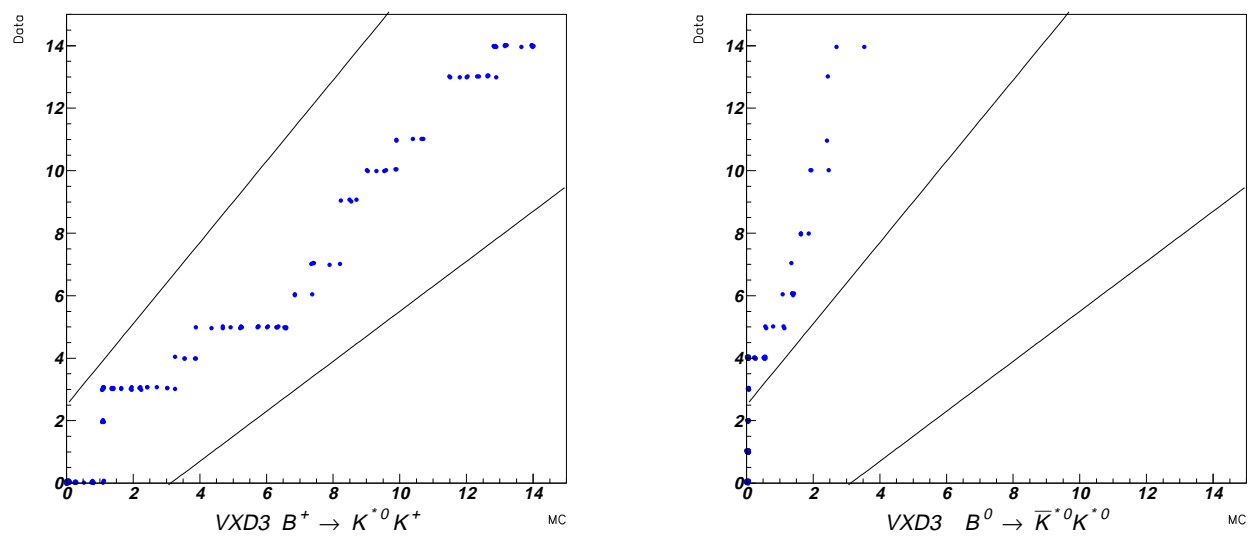

Figure 6.1: Shown are the MC expected background level plotted versus the actual number of events seen in the data. The lines indicates an approximately $90 \%$ confidence interval. A good agreement is seen in the left plot and the MC seems to model this background well. A mismatch is seen in the right plot and it indicates a poor MC modeling of the background level.

eled. For such a study the cut on the discriminator function is gradually made looser and the expected background level is compared to what is actually seen in the data. Figure 6.1 shows two plots from the VXD3 period where expected background (horizontal axis) is compared to data (vertical axis). The lines indicate approximately a $90 \%$ confidence interval. The left plot shows a very well matched behavior for $B^{+} \rightarrow K^{* 0} K^{+}$and it seems that the modeling in MC does a very good job. This is generally true for all the $B \rightarrow P P$ and $B \rightarrow P V$ modes as can be seen in Appendix B Figures B.21 to B.26. The right plot in 6.1, however, shows a rather poor match between data and MC and it seems as if the estimated background is 


\section{Data and Final Results}

too low. Although this plot which is for $B^{0} \rightarrow \bar{K}^{* 0} K^{* 0}$, is rather extreme in its deviation between data and MC it shows a general mismatch for the $B \rightarrow V V$ as can be seen in Appendix B Figures B.23 to B.28 Based on the above considerations, no measurements of rare $B$ decays will be claimed and a upper limit branching ratio will be calculated for each mode in section 6.3 .

A closer look at the data will still be given and as a starter, neither of the tracks in the events are positively identified as protons, muons or electrons. Two of the tracks in $E_{1}$ are identified as pions and in $E_{2}$ two tracks are positively identified, one as a pion and one as a kaon. Further, there is one pion in $E_{3}$ and for $E_{4}$ there are two kaons and one pion. Hence, these identifications are not sufficient to rule out any of the events and the decision of not using the particle ID in a veto mode is thus reinforced.

Table 5.10 in Chapter 5 lists a set of background events as found in the MC. Three of these have two leptons tagged in the $J / \psi(1 s)$ decay which are used in the reconstruction of the $B$ vertex. If any of the data events are of a similar type, two of the tracks will combine to give the $J / \psi$ mass $(3.1 \mathrm{GeV})$. In $E_{1}$, the two track combination that gives a possible $J / \psi$ mass comes out as $3.4 \mathrm{GeV}$, for either muon or electron masses assigned to the tracks. Given the full width $\Gamma=87 \mathrm{KeV}$ for $J / \psi$ this is an unlikely scenario, but it depends somewhat of how well the $J / \psi$ mass is 

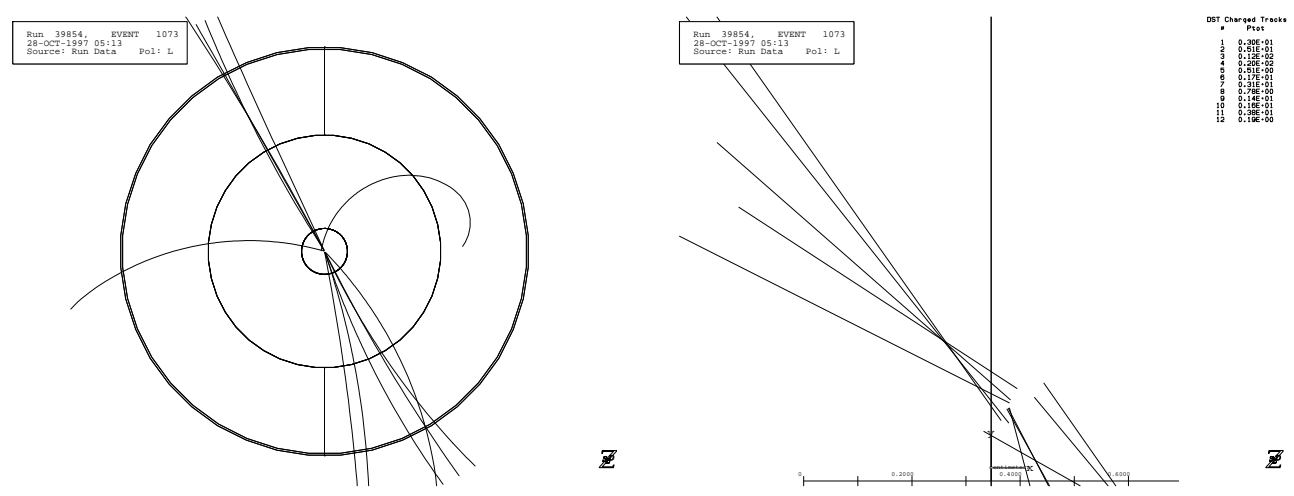

Figure 6.2: Shown are the reconstructed data event $E_{1}$. In the left plot the vertex detector, the DC and the LAC are indicated by the circles. A total of twelve charged tracks are reconstructed for the event and the four tracks in the candidate event move outward in the upper left direction. The right plot show these four tracks coming from the same vertex with a separation from the SLD IP.

reconstructed at SLD. A two track mass combination for the $E_{3}$ gives $2.9 \mathrm{GeV}$ and a $J / \psi$ decay is not necessarily ruled out. For $E_{2}$ and $E_{4}$ no combinations give a mass within $1 \mathrm{GeV}$ of $3.1 \mathrm{GeV}$.

The $E_{1}$ event shows a rather strong character of being a rare $B$ decay and it is displayed in Figure 6.2. In the left plot, the vertex detector, the DC and the LAC are indicated by the circles. A total of twelve charged tracks are reconstructed for the event and the four tracks in the candidate event move outward in the upper left direction. The right plot show these four tracks coming from the same vertex with a clear separation from the SLD IP. This mode has been further investigated where some other scenarios are looked at. These scenarios are not directly supported by 


\section{Data and Final Results}

the MC background events and are somewhat hypothetical of nature. If it is a $B^{0} \rightarrow$ $\pi^{-} D^{+} \rightarrow \pi^{-}\left(K^{-} \pi^{+} \pi^{+}\right)$decay, the $D$ meson mass $(1.9 \mathrm{GeV})$ will be reconstructed from a three track combination. The closest such combination gives $2.5 \mathrm{GeV}$ and is ruled out by the $20 \mathrm{MeV}$ width of the $D$ mass reconstruction at SLD. If it is a $B^{0} \rightarrow \pi^{+} D^{*-} \rightarrow \pi^{+}\left(\pi^{-} \bar{D}^{0}\right) \rightarrow \pi^{+} \pi^{-}\left(K^{-} \pi^{+}\right)$decay, a two track combination will give the $D$ mass. The closest such combination gives a mass of $1.6 \mathrm{GeV}$ and this decay scenario is also ruled out. Other hypothetical modes that have been looked at are a set of $B$ decays into a $D$ meson where the $D$ meson further decays into one neutral track which is missed in the analysis. Neutral tracks are reconstructed in the LAC and a total of 40 such tracks are found in the $E_{1}$ event. The reconstruction of neutrals are fairly poor but by taking the outcome for granted, one can look at possible combinations where a neutral track is added to the already four charged tracks. One favorable combination gives a $D$ mass of $1.8 \mathrm{GeV}$ and a $B$ mass of 5.6 $\mathrm{GeV}$ where the $D$ mass is reconstructed from two of the charged tracks and one of the neutral tracks. A corresponding $B$ decay can be $B^{0} \rightarrow \pi^{+} D^{*-} \rightarrow \pi^{+}\left(\pi^{-} D^{0}\right) \rightarrow$ $\pi^{+} \pi^{-}\left(K^{+} \pi^{-} \pi^{0}\right), \pi^{+} \pi^{-}\left(K^{0} \pi^{+} \pi^{-}\right)$or $B^{0} \rightarrow \rho^{0} D^{0} \rightarrow\left(\pi^{+} \pi^{-}\right)\left(K^{0} \pi^{+} \pi^{-}\right)$.

Although, the existence of a neutral track among the decay products is not ruled out and an overall significant signal in general is not seen, it is still the case that $E_{1}$ is a strong candidate for a rare haronic $B$ decay into two vectors, and may serve as 


\subsection{Limits and Uncertainties}

a hint that $B \rightarrow V V$ decays could eventually be seen at a rate higher than current theoretical predictions. However, no claim can be made on this single event.

\subsection{Limits and Uncertainties}

A great deal of literature exists on how to translate experimental results into upper limits, lower limits or regions of confidence intervals. An approach that will be followed here is recommended by the Particle Data Group [1]. It is a two fold approach where the first result to be reported is the Bayesian upper limit that will be evaluated following a rather traditional technique which is slightly modified by including uncertainties of the measured quantities [71]. The second approach will give a $90 \%$ confidence interval based on the classical method (different from a Bayesian interval) with a new ordering principle [72]. The first subsection (6.2.1) will give a short overview of the statistical methods to be used, subsection 6.2 .2 will give a list of systematic uncertainties and section 6.3 will give the results. 


\section{Data and Final Results}

\subsubsection{Statistical Methods}

The underlying probability for finding $n$ events given a true value $\alpha$ for how many events there really are, follows the Poisson probability $P$,

$$
P=\frac{e^{-\alpha} \alpha^{n}}{n !}
$$

In the Bayesian approach then, in the presence of a measurement where $n_{0}$ events are found, this probability collapses into a probability for the true value $\alpha$,

$$
P_{\alpha}=\frac{e^{-\alpha} \alpha^{n_{0}}}{n_{0} !}
$$

which equals $e^{-\alpha}$ if no events are found. The Bayesian confidence interval with 1- $\epsilon$ probability for the true $\alpha$ to be in a region $\left[\alpha_{1}, \alpha_{2}\right]$ is then given by

$$
1-\epsilon=\int_{\alpha_{1}}^{\alpha_{2}} P_{\alpha} d \alpha
$$

where $\epsilon$ is the probability for $\alpha$ to lie outside the interval. The $90 \%$ lower limit $\alpha_{1}$ is found by letting $\alpha_{2} \rightarrow \infty$ and substituting $\epsilon$ with 0.1 . The naive belief embedded in the Bayesian method of a determined probability for the true underlying parameter $\alpha$ has caused large controversies on the philosophical level. Besides this, there is an obvious flaw if zero events are found. In this case the $90 \% \mathrm{CL}$ lower limit $\left(\alpha_{1}=0.105\right)$ is larger than the measurement and is clearly not describing the data very well. The Bayesian upper limit has caused far fewer controversies and is frequently used in 


\subsection{Limits and Uncertainties}

quoting experimental upper limits as allowed for in [1]. Although, it may not be in principle the right way for quoting a result as argued in [72], the Bayesian upper limit will here be given to make comparison possible to previous results and to results yet to come, which will undoubtedly for still some time cast the results into Bayesian upper limits. The $90 \%$ CL upper limit $\alpha_{2}$ is found by again letting $\epsilon$ equals 0.1 and by setting $\alpha_{1}$ equal to 0 ,

$$
0.9=\int_{0}^{\alpha_{2}} P_{\alpha} d \alpha=1-e^{-\alpha_{2}} \sum_{i=0}^{n_{0}} \frac{\alpha_{2}^{i}}{i !} .
$$

It is not always possible to calculate $\alpha_{2}$ analytically from this, but instead $\alpha_{2}$ is generally found by repeated evaluations of the above expression until the $\alpha_{2}$ that gives the best result is determined. In the presence of a mean expected background $u$ the Bayesian probability $P_{\alpha}$ is modified to $P_{\alpha+u}$ and the above expression becomes

$$
\begin{aligned}
0.9 & =\frac{1}{\mathcal{K}} \int_{0}^{\alpha_{2}} P_{\alpha+u} d \alpha=1-\frac{1}{\mathcal{K}} e^{-\left(\alpha_{2}+u\right)} \sum_{i=0}^{n_{0}} \frac{\left(\alpha_{2}+u\right)^{i}}{i !}, \\
\mathcal{K} & =\int_{0}^{\infty} P_{\alpha+u} d \alpha=e^{-u} \sum_{i=0}^{n_{0}} \frac{u^{i}}{i !}
\end{aligned}
$$

where the denominator $\mathcal{K}$ is the normalization, forcing the total probability to equal one. Still, $n_{0}$ is the number of events found by the experiment and $\alpha_{2}$ is the upper limit to be calculated. In the case of zero found events the Bayesian upper limit is insensitive to the level of background and a full understanding of the background 


\section{Data and Final Results}

level is strictly speaking not necessary. In the cases where events are found in the data the upper limit will decrease with an increase in the background level. A general conservative estimate then, is to make sure that the background level is not set too high in the calculation. For the events seen in this analysis the data indicates that the background level is higher than predicted from MC and it is therefore safe to apply the MC estimated background level (Table 6.1) in calculating the limits. A last piece to put together is the inclusion of uncertainties in an upper limit calculation. The upper limit $\alpha_{2}$ is related to the upper limit branching ratio $B r$ through the sensitivity $S$

$$
\alpha_{2}=S \cdot B r
$$

where the sensitivity is the efficiency for tagging an event multiplied by the number of $B$ mesons present in the search sample. The prescription as given in [71] treats the uncertainty in $\alpha_{2}$ as statistical (which is the reason for why the upper limit is calculated from a Poisson distribution in the first place), and the uncertainties in $S$ is treated as a systematic one. The overall uncertainty for the upper limit branching ratio is then found as the solution to the following expression

$$
0.9=\frac{1}{\mathcal{K}} \frac{1}{\sqrt{2 \pi \sigma_{s}^{2}}} \int_{-\infty}^{\infty}\left(\int_{0}^{\alpha_{2}} P_{\alpha+u} d \alpha\right) e^{-\frac{\left(S-S_{0}\right)^{2}}{2 \sigma_{s}^{2}}} d S
$$




\subsection{Limits and Uncertainties}

where $\alpha_{2}=S \cdot B r . \sigma_{S}$ is the uncertainty in $S$ and must be significantly smaller than $S$ itself to justify the integration range $(-\infty, \infty)$. In the limit of $\sigma_{s} \rightarrow 0$ this reproduces equation 6.6 , and in general this weighted probability satisfies the equation with a solution for $\alpha_{2}$ (or $B r$ ) which is larger than the corresponding solution to equation 6.6. In the case when no events are found, equation 6.8 can be solved exactly and gives for the upper limit $\mathrm{Br}$

$$
B r=\frac{S_{0}-\sqrt{S_{0}^{2}-2 \cdot 2.3 \sigma_{s}^{2}}}{\sigma_{s}^{2}}, \quad \lim _{\sigma_{s} \rightarrow 0} B r=\frac{2.3}{S_{0}},
$$

and reproduces the expected result if the uncertainty is negligible. By integrating equation 6.8 a form suitable for numerical computation can be found,

$$
\begin{aligned}
0.9= & 1-\frac{1}{\mathcal{K}} e^{-\left(u+S_{0} B r-B r^{2} \sigma_{s}^{2} / 2\right)} \\
& -\frac{1}{\mathcal{K}} \frac{1}{\sqrt{2 \pi \sigma_{s}^{2}}} \frac{1}{B r} e^{-p\left(u+S_{0} B r\right)^{2}} \frac{1}{p} \sqrt{\frac{\pi}{p}} \sum_{i=1}^{n_{0}} \frac{1}{i !} \frac{1}{2^{i-1}} \frac{d^{i-1}}{d q^{i-1}}\left(q e^{\frac{q^{2}}{p}}\right)
\end{aligned}
$$

where $p^{-1}=\left(2 \sigma_{s}^{2} B r^{2}\right)$ and $q=p\left(u+S_{0} B r\right)-1 / 2$. If zero events are seen $\left(n_{0}=0\right)$ equation 6.9 can readily be reproduced.

A second approach which is called the 'classical method' in the literature will be used in calculating $90 \%$ confidence intervals. It addresses the question that naturally arises in the Bayesian method: How can a measurement determine the probability distribution of the true underlying parameter $\alpha$ ? This question is the 


\section{Data and Final Results}

main argument against the Bayesian method which strongly relies on a knowledge of the true parameter $\alpha$. The classical method avoids this fundamental question alltogether by constructing confidence intervals in a less naive way. Traditionally, the classical confidence intervals have been the accepted way in which high energy physicists report errors on experimental results.

In a paper by Feldman and Cousins [72] the underlying Neyman method [73] is modified with an ordering principle, avoiding certain problems when the results are near physical boundaries of the probability distribution function. This has led the PDG to recommend the classical method to be reinstated when calculating confidence intervals. A short description of the classical method will be given here while the complete algorithm can be found in [72]. Figure 6.3 shows an illustration of the algorithm for the construction of classical confidence intervals. For each true value $\alpha$ (vertical axis) a horizontal line is drawn which covers $90 \%$ of the possible outcomes (horizontal axis). Due to the discreteness of the Poisson distribution each line will not exactly correspond to $90 \%$ intervals but the construction is made conservative by requiring a $90 \%$ or larger interval. These confidence intervals depends on the expected background and the illustration shown in Figure 6.3 includes a 0.3 background level. The endpoints in each interval are not uniquely given by the defining Neyman method and it is here the debate has been centered. In paper [72] an or- 


\subsection{Limits and Uncertainties}

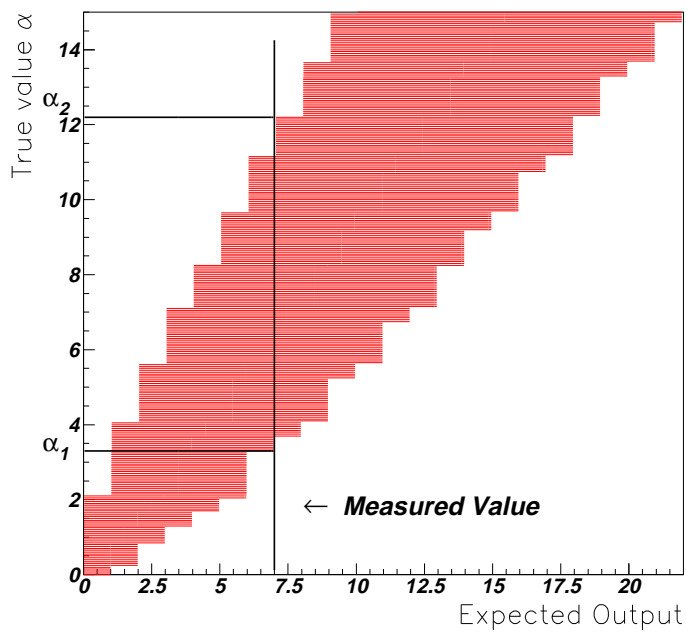

Figure 6.3: Shown are an illustration for the construction of classical confidence intervals in the case of a Poisson distribution.

dering principle is given that seems to address most concerns, if not all, with the classical boundary problem and it can be seen in the Figure that the lower limit is zero including a true value up to 2.1 (with 0.3 background). In the realization of a measurement which in the Figure is exemplified by 7 events, a vertical line is drawn and the lower intersection $\alpha_{1}$ and the upper intersection $\alpha_{2}$ do then satisfy (by construction)

$$
\alpha \in\left[\alpha_{1}, \alpha_{2}\right]
$$

at $90 \%$ confidence and is a statement of the true value $\alpha$. In this example the lower limit $\alpha_{1}=3.30$ and the upper limit $\alpha_{2}=12.2$. 


\section{Data and Final Results}

In the next subsection the sensitivity $S$ and $\sigma_{s}$ will be given for the individual modes and in section 6.3 the Bayesian upper limits will be quoted together with the classical confidence intervals.

\subsubsection{Uncertainties}

The sensitivity as defined in the previous subsection is the product of the efficiency and the number of $B$ mesons produced (Table 1.3), and it remains to estimate the uncertainty on the efficiency numbers (Tables 5.7 and 5.8). The efficiencies are calculated from a limited set of MC generated events which in itself causes a statistical uncertainty. For the VXD3 period and for the $B^{0} \rightarrow \pi^{+} \pi^{-}$mode, for example, there are 4582 events generated from which 1576 events pass the cuts. According to the binomial distribution then, the statistical uncertainty associated with these numbers is

$$
\delta \epsilon=\frac{1}{4582} \sqrt{1576\left(1-\frac{1576}{4582}\right)}=0.007
$$

with $\epsilon=1576 / 4582=0.344$ giving a relative uncertainty of $2 \%$. A second effect causing uncertainties in the efficiency numbers comes from the MC modeling of the modes. Section 4.3 describes a set of corrections applied to the MC events and as a measure for the modeling uncertainties these corrections will be undone. Tables 6.3 


\subsection{Limits and Uncertainties}

\begin{tabular}{lcccccccc}
\multicolumn{3}{c}{ VXD2 } & \multicolumn{1}{c}{ VXD3 } \\
Mode & $\mathcal{N}$ & $p_{t}$ & $\mathcal{T}$ & $\mathcal{R}$ & $\mathcal{N}$ & $p_{t}$ & $\mathcal{T}$ & $\mathcal{R}$ \\
\hline$B^{0} \rightarrow \pi^{+} \pi^{-}$ & 37.8 & 34.4 & 34.3 & 32.5 & 37.3 & 36.3 & 36.1 & 34.4 \\
$B^{0} \rightarrow K^{-} \pi^{+}$ & 36.2 & 33.7 & 33.5 & 31.9 & 38.9 & 37.8 & 37.6 & 35.6 \\
$B^{0} \rightarrow K^{+} K^{-}$ & 35.2 & 32.2 & 32.0 & 29.8 & 38.2 & 36.9 & 36.5 & 35.8 \\
$B_{s} \rightarrow \pi^{+} \pi^{-}$ & 36.0 & 34.1 & 39.9 & 31.6 & 42.7 & 41.2 & 40.6 & 40.3 \\
$B_{s} \rightarrow K^{+} \pi^{-}$ & 36.5 & 33.7 & 33.4 & 31.7 & 36.6 & 35.4 & 35.1 & 34.3 \\
$B_{s} \rightarrow K^{+} K^{-}$ & 34.7 & 31.8 & 31.4 & 29.7 & 33.7 & 32.2 & 31.8 & 31.6 \\
\hline$B^{+} \rightarrow \rho^{0} \pi^{+}$ & 27.7 & 26.1 & 25.6 & 24.0 & 31.2 & 30.1 & 29.3 & 28.5 \\
$B^{+} \rightarrow \rho^{0} K^{+}$ & 24.9 & 23.1 & 22.2 & 21.6 & 30.7 & 29.7 & 29.0 & 28.3 \\
$B^{+} \rightarrow K^{* 0} \pi^{+}$ & 26.4 & 24.7 & 24.4 & 22.7 & 33.3 & 32.4 & 31.6 & 30.8 \\
$B^{+} \rightarrow K^{* 0} K^{+}$ & 25.0 & 23.5 & 21.9 & 19.2 & 29.5 & 28.5 & 27.9 & 27.0 \\
$B^{+} \rightarrow \phi \pi^{+}$ & 31.3 & 29.7 & 29.4 & 28.0 & 33.1 & 32.3 & 31.8 & 30.9 \\
$B^{+} \rightarrow \phi K^{+}$ & 31.4 & 28.7 & 27.6 & 27.1 & 35.8 & 34.8 & 34.5 & 34.0 \\
\hline
\end{tabular}

Table 6.3: Shown are the effects on the efficiencies as a result of the corrections applied on the MC generated events. The $\mathcal{N}$ column shows the efficiencies if no corrections are applied and the $\mathcal{R}$ shows the efficiencies after all corrections. The difference between the endpoints $(\mathcal{N}-\mathcal{R})$ will be used as systematic uncertainty for the final efficiency $(\mathcal{R})$.

and 6.4 show the effect of applying the various corrections. First, the uncorrected MC efficiencies are listed $\mathcal{N}$, second when the $p_{t}$ smearing is applied, third when the tracking corrections $\mathcal{T}$ are added and last when the resolution corrections $\mathcal{R}$ are applied. The difference between the endpoints $(\mathcal{N}-\mathcal{R})$ will be used as systematic uncertainty for the final efficiency $(\mathcal{R})$. Tables 6.5 and 6.6 lists this systematic uncertainty $\delta \epsilon_{s y s}$ together with the statistical uncertainty $\delta \epsilon_{\text {sta }}$ which is caused by the limited MC sample as described above. These are then added in quadrature, combined together for VXD2 and VXD3 and cast into an combined uncertainty 


\section{Data and Final Results}

\begin{tabular}{lcccccccc} 
& VXD2 & \multicolumn{7}{c}{ VXD3 } \\
Mode & $\mathcal{N}$ & $p_{t}$ & $\mathcal{T}$ & $\mathcal{R}$ & $\mathcal{N}$ & $p_{t}$ & $\mathcal{T}$ & $\mathcal{R}$ \\
\hline$B^{0} \rightarrow \rho^{0} \rho^{0}$ & 27.6 & 26.5 & 26.2 & 24.5 & 30.3 & 29.3 & 28.4 & 27.9 \\
$B^{0} \rightarrow \bar{K}^{* 0} \rho^{0}$ & 22.0 & 21.5 & 21.3 & 19.4 & 30.8 & 29.9 & 28.8 & 28.4 \\
$B^{0} \rightarrow \bar{K}^{* 0} K^{* 0}$ & 27.4 & 27.2 & 26.7 & 24.5 & 36.3 & 35.2 & 34.2 & 33.8 \\
$B^{0} \rightarrow \phi \rho^{0}$ & 29.3 & 28.4 & 27.8 & 25.6 & 34.9 & 33.8 & 32.9 & 32.4 \\
$B^{0} \rightarrow \phi \bar{K}^{* 0}$ & 27.1 & 26.9 & 26.2 & 25.3 & 34.1 & 33.2 & 32.5 & 32.2 \\
$B^{0} \rightarrow \phi \phi$ & 27.2 & 27.0 & 26.5 & 25.8 & 33.1 & 32.9 & 32.6 & 31.6 \\
\hline$B_{s} \rightarrow \rho^{0} \rho^{0}$ & 27.1 & 26.8 & 26.4 & 23.9 & 33.2 & 32.6 & 31.1 & 30.1 \\
$B_{s} \rightarrow \bar{K}^{* 0} \rho^{0}$ & 26.3 & 24.5 & 23.9 & 22.8 & 32.4 & 31.0 & 30.2 & 29.9 \\
$B_{s} \rightarrow \bar{K}^{* 0} K^{* 0}$ & 22.3 & 22.0 & 21.4 & 20.8 & 30.9 & 30.2 & 30.0 & 29.8 \\
$B_{s} \rightarrow \phi \rho^{0}$ & 27.9 & 26.4 & 26.3 & 23.6 & 33.2 & 32.6 & 32.4 & 32.1 \\
$B_{s} \rightarrow \phi K^{* 0}$ & 28.4 & 27.6 & 27.4 & 25.4 & 30.6 & 29.8 & 29.2 & 28.0 \\
$B_{s} \rightarrow \phi \phi$ & 28.8 & 28.1 & 27.6 & 25.4 & 35.5 & 35.2 & 34.6 & 34.0 \\
\hline
\end{tabular}

Table 6.4: Shown are the effects on the efficiencies as a result of the corrections applied on the MC generated events. The $\mathcal{N}$ column shows the efficiencies if no corrections are applied and the $\mathcal{R}$ shows the efficiencies after all corrections. The difference between the end points $(\mathcal{N}-\mathcal{R})$ will be used as systematic uncertainty for the final efficiency $(\mathcal{R})$.

$\delta \epsilon$. The uncertainty $\sigma_{s}$ for the sensitivity $S$ is then listed where the uncertainty from the $B$ sample size is included (Table 1.3 in Chapter 1). Further, for modes including a vector meson decaying into a combinations of charged and neutral modes the sensitivity has been adjusted due to the insensitivity of tagging neutral modes (subsection 1.3.1). For $B_{s}$ modes the $B$ sample size uncertainty contributes by around the same amount as the efficiency uncertainties while for other modes the $\sigma_{s}$ is mainly dominated by $\delta \epsilon$. 


\subsection{Limits and Uncertainties}

\begin{tabular}{lccccccc} 
& VXD2 & \multicolumn{3}{c}{ VXD3 } & \multicolumn{3}{c}{ Combined } \\
Mode & $\delta \epsilon_{\text {sys }}$ & $\delta \epsilon_{\text {sta }}$ & $\delta \epsilon_{\text {sys }}$ & $\delta \epsilon_{\text {sta }}$ & $\delta \epsilon$ & $S$ & $\sigma_{s}$ \\
\hline$B^{0} \rightarrow \pi^{+} \pi^{-}$ & 5.3 & 0.7 & 2.9 & 0.7 & 2.7 & 34615 & 3097 \\
$B^{0} \rightarrow K^{-} \pi^{+}$ & 4.3 & 0.7 & 3.3 & 0.7 & 2.8 & 35323 & 3201 \\
$B^{0} \rightarrow K^{+} K^{-}$ & 5.4 & 0.4 & 2.4 & 0.4 & 2.4 & 34866 & 2844 \\
$B_{s} \rightarrow \pi^{+} \pi^{-}$ & 4.4 & 1.4 & 2.4 & 1.1 & 2.4 & 10232 & 1644 \\
$B_{s} \rightarrow K^{+} \pi^{-}$ & 4.8 & 1.2 & 2.3 & 0.8 & 2.3 & 9094 & 1481 \\
$B_{s} \rightarrow K^{+} K^{-}$ & 5.0 & 1.2 & 2.1 & 0.8 & 2.3 & 8398 & 1379 \\
\hline$B^{+} \rightarrow \rho^{0} \pi^{+}$ & 3.7 & 0.4 & 2.7 & 0.4 & 2.3 & 27836 & 2599 \\
$B^{+} \rightarrow \rho^{0} K^{+}$ & 3.3 & 0.8 & 2.4 & 0.4 & 2.1 & 26998 & 2378 \\
$B^{+} \rightarrow K^{* 0} \pi^{+}$ & 3.7 & 0.6 & 2.5 & 0.4 & 2.2 & 19434 & 1683 \\
$B^{+} \rightarrow K^{* 0} K^{+}$ & 5.8 & 0.6 & 2.5 & 0.4 & 2.5 & 16902 & 1837 \\
$B^{+} \rightarrow \phi \pi^{+}$ & 3.3 & 0.8 & 2.2 & 0.6 & 2.0 & 15098 & 1171 \\
$B^{+} \rightarrow \phi K^{+}$ & 4.3 & 0.8 & 1.8 & 0.6 & 2.0 & 16089 & 1176 \\
\hline
\end{tabular}

Table 6.5: Shown are the efficiency uncertainties, the sensitivity $S$ with its standard deviation $\sigma_{s}$ fot $B \rightarrow P P$ and $B \rightarrow P V$.

\begin{tabular}{lccccccc} 
& VXD2 & \multicolumn{5}{c}{ VXD3 } & \multicolumn{3}{c}{ Combined } \\
Mode & $\delta \epsilon_{\text {sys }}$ & $\delta \epsilon_{\text {sta }}$ & $\delta \epsilon_{\text {sys }}$ & $\delta \epsilon_{\text {sta }}$ & $\delta \epsilon$ & $S$ & $\sigma_{s}$ \\
\hline$B^{0} \rightarrow \rho^{0} \rho^{0}$ & 3.1 & 0.7 & 2.4 & 0.3 & 2.0 & 27539 & 2356 \\
$B^{0} \rightarrow \bar{K}^{* 0} \rho^{0}$ & 2.6 & 0.9 & 2.4 & 0.4 & 2.0 & 17626 & 1529 \\
$B^{0} \rightarrow \bar{K}^{* 0} K^{* 0}$ & 2.9 & 1.1 & 2.5 & 1.0 & 2.2 & 14165 & 1163 \\
$B^{0} \rightarrow \phi \rho^{0}$ & 3.7 & 1.1 & 2.5 & 1.0 & 2.3 & 15300 & 1308 \\
$B^{0} \rightarrow \phi \bar{K}^{* 0}$ & 1.8 & 1.3 & 1.9 & 1.2 & 1.8 & 10123 & 737 \\
$B^{0} \rightarrow \phi \phi$ & 1.4 & 1.5 & 1.5 & 1.4 & 1.7 & 7384 & 512 \\
\hline$B_{s} \rightarrow \rho^{0} \rho^{0}$ & 3.2 & 1.4 & 3.1 & 1.5 & 2.7 & 7665 & 1349 \\
$B_{s} \rightarrow \bar{K}^{* 0} \rho^{0}$ & 3.5 & 1.7 & 2.5 & 1.4 & 2.4 & 5028 & 857 \\
$B_{s} \rightarrow \bar{K}^{* 0} K^{* 0}$ & 1.5 & 1.8 & 1.1 & 1.3 & 1.5 & 3276 & 515 \\
$B_{s} \rightarrow \phi \rho^{0}$ & 4.3 & 1.8 & 1.1 & 1.5 & 2.0 & 3943 & 638 \\
$B_{s} \rightarrow \phi K^{* 0}$ & 3.0 & 2.0 & 2.6 & 1.5 & 2.5 & 2413 & 416 \\
$B_{s} \rightarrow \phi \phi$ & 3.4 & 1.9 & 1.5 & 1.6 & 2.0 & 2058 & 331 \\
\hline
\end{tabular}

Table 6.6: Shown are the efficiency uncertainties, the sensitivity $S$ with its standard deviation $\sigma_{s}$ for $B \rightarrow V V$. 


\section{Data and Final Results}

\subsection{Results}

In table 6.7 the Bayesian $90 \%$ confidence level upper limit for the branching ratios are shown in units of $10^{-4}$. The limits range from $0.6 \cdot 10^{-4}$ for $B^{0} \rightarrow P P$ to $17 \cdot 10^{-4}$ for $B_{s} \rightarrow \bar{K}^{* 0} K^{* 0}$, where the two events in the data combined with a low sensitivity forces the upper limit to be large. The decrease in these limit is shown if the uncertainty in the sensitivity is ignored $\left(\sigma_{s}=0\right)$. The relative effect on the upper limit of a nonzero $\sigma_{s}$ varies between $1-4 \%$ for all modes and is significantly smaller than the relative size of $\sigma_{s}$ to $S$. Hence, this shows that the errors are not

\begin{tabular}{|c|c|c|c|c|c|c|c|}
\hline Mode & $\begin{array}{l}\left(\times 10^{4}\right) \\
\text { UL } B r\end{array}$ & $\begin{array}{c}\sigma_{s}=0 \\
\delta B r\end{array}$ & $\begin{array}{c}u=0 \\
\delta B r\end{array}$ & Mode & $\begin{array}{l}\left(\times 10^{4}\right) \\
\text { UL } B r\end{array}$ & $\begin{array}{c}\sigma_{s}=0 \\
\delta B r\end{array}$ & $\begin{array}{c}u=0 \\
\delta B r\end{array}$ \\
\hline$B^{0} \rightarrow \pi^{+} \pi^{-}$ & 0.67 & -0.01 & 0.00 & $B^{0} \rightarrow \rho^{0} \rho^{0}$ & 1.33 & -0.01 & +0.10 \\
\hline$B^{0} \rightarrow K^{-} \pi^{+}$ & 0.66 & -0.01 & 0.00 & $B^{0} \rightarrow \bar{K}^{* 0} \rho^{0}$ & 2.79 & -0.03 & +0.27 \\
\hline$B^{0} \rightarrow K^{+} K^{-}$ & 0.66 & -0.01 & 0.00 & $B^{0} \rightarrow \bar{K}^{* 0} K^{* 0}$ & 4.58 & -0.06 & +0.18 \\
\hline$B_{s} \rightarrow \pi^{+} \pi^{-}$ & 2.32 & -0.07 & 0.00 & $B^{0} \rightarrow \phi \rho^{0}$ & 1.52 & -0.01 & 0.00 \\
\hline$B_{s} \rightarrow K^{+} \pi^{-}$ & 2.61 & -0.08 & 0.00 & $B^{0} \rightarrow \phi \bar{K}^{* 0}$ & 3.75 & -0.03 & +0.03 \\
\hline$B_{s} \rightarrow K^{+} K^{-}$ & 2.83 & -0.09 & 0.00 & $B^{0} \rightarrow \phi \phi$ & 3.13 & -0.02 & 0.00 \\
\hline$B^{+} \rightarrow \rho^{0} \pi^{+}$ & 0.83 & -0.01 & 0.00 & $B_{s} \rightarrow \rho^{0} \rho^{0}$ & 3.12 & -0.12 & 0.00 \\
\hline$B^{+} \rightarrow \rho^{0} K^{+}$ & 0.86 & -0.01 & 0.00 & $B_{s} \rightarrow \bar{K}^{* 0} \rho^{0}$ & 7.48 & -0.31 & +0.61 \\
\hline$B^{+} \rightarrow K^{* 0} \pi^{+}$ & 1.19 & -0.01 & 0.00 & $B_{s} \rightarrow \bar{K}^{* 0} K^{* 0}$ & 16.40 & -0.69 & +0.23 \\
\hline$B^{+} \rightarrow K^{* 0} K^{+}$ & 1.38 & -0.02 & 0.00 & $B_{s} \rightarrow \phi \rho^{0}$ & 6.02 & -0.19 & 0.00 \\
\hline$B^{+} \rightarrow \phi \pi^{+}$ & 1.53 & -0.01 & 0.00 & $B_{s} \rightarrow \phi K^{* 0}$ & 9.88 & -0.35 & 0.00 \\
\hline$B^{+} \rightarrow \phi K^{+}$ & 1.44 & -0.01 & 0.00 & $B_{s} \rightarrow \phi \phi$ & 11.54 & -0.35 & 0.00 \\
\hline
\end{tabular}

Table 6.7: The Bayesian 90\% confidence level upper limit for the branching ratios are shown in units of $10^{-4}$. The decrease in this limit is shown if the uncertainty in the sensitivity is ignored $\left(\sigma_{s}=0\right)$. Modes that do see events in data are responsive to the background level and the increase in the upper limit is shown if the background is set to zero $(u=0)$. 


\subsection{Results}

\begin{tabular}{lccc} 
& Bckg. $=u$ & Bckg.=u/2 & Bckg.=4u \\
Mode & $B r$ & $B r$ & $B r$ \\
$B^{0} \rightarrow \pi^{+} \pi^{-}$ & $0.00-0.69$ & $0.00-0.69$ & $0.00-0.66$ \\
$B^{0} \rightarrow K^{-} \pi^{+}$ & $0.00-0.65$ & $0.00-0.67$ & $0.00-0.54$ \\
$B^{0} \rightarrow K^{+} K^{-}$ & $0.00-0.66$ & $0.00-0.67$ & $0.00-0.54$ \\
$B_{s} \rightarrow \pi^{+} \pi^{-}$ & $0.00-2.35$ & $0.00-2.35$ & $0.00-2.25$ \\
$B_{s} \rightarrow K^{+} \pi^{-}$ & $0.00-2.56$ & $0.00-2.62$ & $0.00-2.45$ \\
$B_{s} \rightarrow K^{+} K^{-}$ & $0.00-2.66$ & $0.00-2.77$ & $0.00-2.04$ \\
\hline$B^{+} \rightarrow \rho^{0} \pi^{+}$ & $0.00-0.75$ & $0.00-0.81$ & $0.00-0.51$ \\
$B^{+} \rightarrow \rho^{0} K^{+}$ & $0.00-0.75$ & $0.00-0.83$ & $0.00-0.47$ \\
$B^{+} \rightarrow K^{* 0} \pi^{+}$ & $0.00-1.16$ & $0.00-1.21$ & $0.00-0.92$ \\
$B^{+} \rightarrow K^{* 0} K^{+}$ & $0.00-1.34$ & $0.00-1.39$ & $0.00-1.06$ \\
$B^{+} \rightarrow \phi \pi^{+}$ & $0.00-1.56$ & $0.00-1.59$ & $0.00-1.42$ \\
$B^{+} \rightarrow \phi K^{+}$ & $0.00-1.42$ & $0.00-1.47$ & $0.00-1.17$ \\
\hline
\end{tabular}

Table 6.8: Shown are the $90 \%$ classical confidence intervals with three choices for the background level Bckg. $u$ is the MC estimated value as listed in Table 6.1

propagated through in the calculation in the same way as one expects for a central value result. Modes that do have events in the data are responsive to the background level and the increase in the upper limit is shown if the background is set to zero $(u=0)$. As argued before, the estimated background level appears to be smaller than what is indicated by data and the upper limit results for the modes sensitive to background is conservatively set with respect to the influence from backgrounds.

Tables 6.8 and 6.9 show the classical $90 \%$ confidence intervals. Due to the lower and the upper boundary of the interval, the background levels must in principle be accurately measured since there is no conservative way of treating the background. If the background is set too high the upper value of the interval becomes too small 


\section{Data and Final Results}

\begin{tabular}{lccc} 
& Bckg. $=u$ & Bckg. $=u / 2$ & Bckg. $=4 u$ \\
Mode & $B r$ & $B r$ & $B r$ \\
$B^{0} \rightarrow \rho^{0} \rho^{0}$ & $0.00-1.47$ & $0.00-1.53$ & $0.00-1.14$ \\
$B^{0} \rightarrow \bar{K}^{* 0} \rho^{0}$ & $0.03-3.08$ & $0.17-3.22$ & $0.00-2.26$ \\
$B^{0} \rightarrow \bar{K}^{* 0} K^{* 0}$ & $0.59-5.05$ & $0.68-5.14$ & $0.01-4.47$ \\
$B^{0} \rightarrow \phi \rho^{0}$ & $0.00-1.50$ & $0.00-1.54$ & $0.00-1.23$ \\
$B^{0} \rightarrow \phi \bar{K}^{* 0}$ & $0.00-4.17$ & $0.04-4.23$ & $0.00-3.75$ \\
$B^{0} \rightarrow \phi \phi$ & $0.00-3.29$ & $0.00-3.29$ & $0.00-3.29$ \\
\hline$B_{s} \rightarrow \rho^{0} \rho^{0}$ & $0.00-2.82$ & $0.00-2.99$ & $0.00-2.02$ \\
$B_{s} \rightarrow \bar{K}^{* 0} \rho^{0}$ & $0.00-7.98$ & $0.00-8.31$ & $0.00-5.99$ \\
$B_{s} \rightarrow \bar{K}^{* 0} K^{* 0}$ & $1.13-17.52$ & $1.37-17.77$ & $0.00-15.96$ \\
$B_{s} \rightarrow \phi \rho^{0}$ & $0.00-5.99$ & $0.00-6.09$ & $0.00-5.45$ \\
$B_{s} \rightarrow \phi K^{* 0}$ & $0.00-9.49$ & $0.00-9.78$ & $0.00-7.79$ \\
$B_{s} \rightarrow \phi \phi$ & $0.00-11.81$ & $0.00-11.81$ & $0.00-11.81$ \\
\hline
\end{tabular}

Table 6.9: Shown are the $90 \%$ classical confidence intervals with three choices for the background level Bckg. $u$ is the MC estimated value as listed in Table 6.1

and if the background level is set too low the lower value of the interval becomes too high. To estimate this effect, three different intervals are calculated based upon three different background levels, one where the background is as estimated by the MC, second where the background is reduced by a factor of 2 and third, where the background is increased by a factor of 4 . This covers the range of mismatch as is seen between data and $\mathrm{MC}$ when the cuts are made looser (Figures B.21 to B.28). As opposed to the Bayesian method, it can be seen that the modes that do not have any observed events are also sensitive to the background level. The effect of the uncertainty in the sensitivity is not included since it gives a relatively small effect which should be picked up by the larger effect from changing the background levels. 


\subsection{Results}

Due to the uncertainty in the estimated background as indicated from data in certain modes a conservative quoted classical confidence interval that allows for a range of background levels will then be the union of the three intervals given in Tables 6.8 and 6.9 .

A point of particular interest is the Delphi result for the combined branching ratio for the modes $B^{+} \rightarrow \rho^{0} \pi^{+}$and $B^{+} \rightarrow K^{* 0} \pi^{+}$which is quoted as

$$
\operatorname{Br}\left(B^{+} \rightarrow \rho \pi^{+}, K^{* 0} \pi^{+}\right)=\left(17_{-8}^{+12} \pm 2\right) 10^{-5}
$$

A relevant consideration is thus the degree of overlap between the two results. The Delphi result is based upon three events in the data with near zero background and gives a sensitivity of $S_{D}=3 \cdot 10^{4} / 1.7=17647$. The corresponding Bayesian probability distribution is given by

$$
P_{D}\left(B r_{D}\right)=\frac{e^{-B r_{D} S_{D}}\left(B r_{D} S_{D}\right)^{r} S_{D}}{r !}
$$

where $r=3$ and the probability distribution has been formulated as a probability distribution for the Branching ratio at Delphi $B r_{D}$. At SLD the combined sensitivity for these modes is $S_{S}=23635$ and the probability distribution for the branching ratio is

$$
P_{S}=e^{-B r_{S} S_{S}} S_{S}
$$




\section{Data and Final Results}

Now, by change of coordinates

$$
\begin{aligned}
& y=R_{D}-R_{S} \quad y \in\langle-\infty, \infty\rangle, \\
& \rho=\frac{R_{D}+R_{S}}{2}, \quad \rho \in\left[\frac{|y|}{2}, \infty\right\rangle,
\end{aligned}
$$

a distribution function $\mathcal{F}(y)$ of the difference $y$ between the two branching ratio results at SLD and Delphi can be constructed

$$
\mathcal{F}(y)=\int_{\frac{|y|}{2}}^{\infty} P_{S}\left(R_{S}\right) P_{D}\left(R_{D}\right) d \rho=\int_{\frac{|y|}{2}}^{\infty} P_{S}\left(\frac{2 \rho-y}{2}\right) P_{D}\left(\frac{2 \rho+y}{2}\right) d \rho .
$$

The solution to this integral is

$$
\begin{array}{ll}
\mathcal{F}(y)=\frac{S_{D}^{(r+1)} S_{S} e^{S_{S} y}}{\left(S_{S}+S_{D}\right)^{r+1}} & y<0 \\
\mathcal{F}(y)=\frac{S_{D}^{(r+1)} S_{S} e^{-S_{D} y}}{\left(S_{S}+S_{D}\right)^{r+1}} \sum_{i=0}^{r} \frac{\left[\left(S_{S}+S_{D}\right) y\right]^{i}}{i !} & y>0,
\end{array}
$$

with $r=3$, and can be used to quantify the degree the SLD result excludes the Delphi result. The mean difference $\langle y\rangle$ is given by

$$
\langle y\rangle=\int_{-\infty}^{\infty} \mathcal{F}(y) y d y=\frac{r S_{S}+S_{S}-S_{D}}{S_{S} S_{D}}=1.84 \cdot 10^{-4}
$$

and in Figure 6.4 this value is shown in a plot of $\mathcal{F}(y)$. The amount of exclusion between the two measurements are then the two areas $A_{1}(y \in[0,\langle y\rangle])$ and $A_{2}$ $(y \in[\langle y\rangle, 2\langle y\rangle])$. This approach is in direct analog with the treatment of Gaussian 


\subsection{Results}

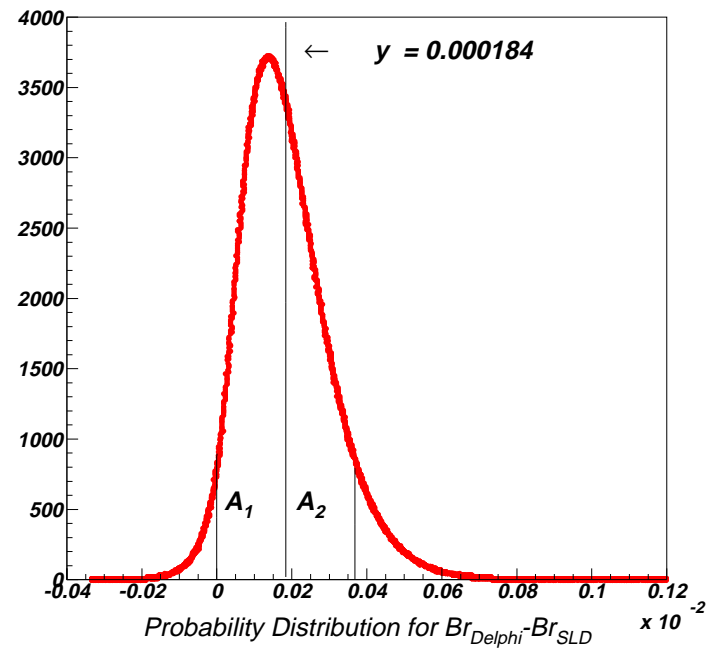

Figure 6.4: Shown are the probability distribution for the Delphi branching ratio minus the SLD branching ratio for the combined process $B^{+} \rightarrow \rho \pi^{+}, K^{* 0} \pi^{+}$.

variables when the amount of exclusion is calculated ${ }^{1}$ and it is found that the indicated area equals $89.9 \%$. Hence, the SLD result excludes the Delphi result with $90 \%$ confidence giving more weight to the CLEO result for these modes. In the Gaussian analog the difference between the SLD and Delphi measurements is $1.6 \sigma$.

\subsubsection{Summary}

The SLD experiment has provided competitive upper limit branching ratios for hadronic rare $B$ decays. This has become possible by exploiting the excellent ver-

\footnotetext{
${ }^{1}$ The direct interpretation of this approach can be view as a quantitative statement of the probability for a change in $y$ such that the measurements agree.
} 


\section{Data and Final Results}

texing at SLD and by use of a novel event selection algorithm, utilizing direct cuts together with discriminator functions. Efficiencies around $30 \%$ have been achieved with near zero background. This greatly compensates for a relative low statistical data sample and new and improved upper limits for $B$ decays into two vector particles have been given. The results are consistent with theoretical predictions and a Delphi measurement for the combined decay of $B^{+}$into $\rho^{0} \pi^{+}$and $K^{* 0} \pi^{+}$has been excluded with $90 \%$ confidence. Table 6.10 summaries the result of this search with both Bayesian 90\% confidence level upper limits (CL UL) and classical $90 \%$ confidence level intervals (CL I). The expected background levels are also listed together with the efficiencies. The listed efficiencies are for charged final states only and must be reduced for modes that include $\phi$ or $K^{* 0}$ as explained in Chapter 1 . 


\subsection{Results}

\begin{tabular}{lccccc} 
& & & Events & Classical 90\% & Bayesian $90 \%$ \\
Mode & $\varepsilon$ & Bckg. & in Data & CL I $\left(\times 10^{4}\right)$ & CL UL $\left(\times 10^{4}\right)$ \\
\hline$B^{0} \rightarrow \pi^{+} \pi^{-}$ & 0.338 & 0.03 & 0 & $0.00-0.69$ & 0.67 \\
$B^{0} \rightarrow K^{-} \pi^{+}$ & 0.345 & 0.14 & 0 & $0.00-0.67$ & 0.66 \\
$B^{0} \rightarrow K^{+} K^{-}$ & 0.341 & 0.14 & 0 & $0.00-0.67$ & 0.66 \\
$B_{s} \rightarrow \pi^{+} \pi^{-}$ & 0.379 & 0.03 & 0 & $0.00-2.35$ & 2.32 \\
$B_{s} \rightarrow K^{+} \pi^{-}$ & 0.335 & 0.10 & 0 & $0.00-2.62$ & 2.61 \\
$B_{s} \rightarrow K^{+} K^{-}$ & 0.311 & 0.20 & 0 & $0.00-2.77$ & 2.83 \\
\hline$B^{+} \rightarrow \rho^{0} \pi^{+}$ & 0.272 & 0.34 & 0 & $0.00-0.81$ & 0.83 \\
$B^{+} \rightarrow \rho^{0} K^{+}$ & 0.264 & 0.41 & 0 & $0.00-0.83$ & 0.86 \\
$B^{+} \rightarrow K^{* 0} \pi^{+}$ & 0.285 & 0.17 & 0 & $0.00-1.21$ & 1.19 \\
$B^{+} \rightarrow K^{* 0} K^{+}$ & 0.248 & 0.17 & 0 & $0.00-1.39$ & 1.38 \\
$B^{+} \rightarrow \phi \pi^{+}$ & 0.301 & 0.07 & 0 & $0.00-1.59$ & 1.53 \\
$B^{+} \rightarrow \phi K^{+}$ & 0.321 & 0.14 & 0 & $0.00-1.47$ & 1.44 \\
\hline$B^{0} \rightarrow \rho^{0} \rho^{0}$ & 0.270 & 0.31 & 1 & $0.00-1.53$ & 1.33 \\
$B^{0} \rightarrow \bar{K}^{* 0} \rho^{0}$ & 0.259 & 0.49 & 2 & $0.00-3.22$ & 2.79 \\
$B^{0} \rightarrow \bar{K}^{* 0} K^{* 0}$ & 0.312 & 0.27 & 3 & $0.01-5.14$ & 4.58 \\
$B^{0} \rightarrow \phi \rho^{0}$ & 0.305 & 0.14 & 0 & $0.00-1.54$ & 1.52 \\
$B^{0} \rightarrow \phi \bar{K}^{* 0}$ & 0.302 & 0.14 & 1 & $0.00-4.23$ & 3.75 \\
$B^{0} \rightarrow \phi \phi$ & 0.300 & 0.00 & 0 & $0.00-3.29$ & 3.13 \\
\hline$B_{s} \rightarrow \rho^{0} \rho^{0}$ & 0.284 & 0.27 & 0 & $0.00-2.99$ & 3.12 \\
$B_{s} \rightarrow \bar{K}^{* 0} \rho^{0}$ & 0.279 & 0.34 & 1 & $0.00-8.31$ & 7.48 \\
$B_{s} \rightarrow \bar{K}^{* 0} K^{* 0}$ & 0.272 & 0.17 & 2 & $0.00-17.77$ & 16.40 \\
$B_{s} \rightarrow \phi \rho^{0}$ & 0.297 & 0.07 & 0 & $0.00-6.09$ & 6.02 \\
$B_{s} \rightarrow \phi K^{* 0}$ & 0.272 & 0.14 & 0 & $0.00-9.78$ & 9.88 \\
$B_{s} \rightarrow \phi \phi$ & 0.316 & 0.00 & 0 & $0.00-11.81$ & 11.54 \\
\hline & & & & & \\
\hline
\end{tabular}

Table 6.10: Shown are the final results of the search. The number of events found in the data are displayed with Bayesian upper limits and classical confidence intervals as explained in the text. The expected background levels are also listed together with the efficiencies. The listed efficiencies are for charged final states only and must be reduced for modes that include $\phi$ or $K^{* 0}$ as explained in Chapter 1. 

$\prod_{\text {Appendix }}$

\section{The SLD Collaboration}

Kenji Abe, ${ }^{(21)}$ Koya Abe, ${ }^{(33)}$ T. Abe, ${ }^{(29)}$ I. Adam, ${ }^{(29)}$ T. Akagi, ${ }^{(29)}$ H. Akimoto, ${ }^{(29)}$

N.J. Allen, ${ }^{(5)}$ W.W. Ash, ${ }^{(29)}$ D. Aston, ${ }^{(29)}$ K.G. Baird, ${ }^{(17)}$ C. Baltay, ${ }^{(40)}$

H.R. Band, ${ }^{(39)}$ M.B. Barakat, ${ }^{(16)}$ O. Bardon, ${ }^{(19)}$ T.L. Barklow, ${ }^{(29)}$

G.L. Bashindzhagyan, ${ }^{(20)}$ J.M. Bauer, ${ }^{(18)}$ G. Bellodi, ${ }^{(23)}$ A.C. Benvenuti, ${ }^{(3)}$

G.M. Bilei, ${ }^{(25)}$ D. Bisello, ${ }^{(24)}$ G. Blaylock, ${ }^{(17)}$ J.R. Bogart, ${ }^{(29)}$ G.R. Bower, ${ }^{(29)}$

J.E. Brau, ${ }^{(22)}$ M. Breidenbach, ${ }^{(29)}$ W.M. Bugg, ${ }^{(32)}$ D. Burke, ${ }^{(29)}$ T.H. Burnett, ${ }^{(38)}$

P.N. Burrows, ${ }^{(23)}$ A. Calcaterra, ${ }^{(12)}$ D. Calloway, ${ }^{(29)}$ B. Camanzi, ${ }^{(11)}$

M. Carpinelli, ${ }^{(26)}$ R. Cassell, ${ }^{(29)}$ R. Castaldi, ${ }^{(26)}$ A. Castro, ${ }^{(24)}$ M. Cavalli-Sforza, ${ }^{(35)}$

A. Chou, ${ }^{(29)}$ E. Church, ${ }^{(38)}$ H.O. Cohn, ${ }^{(32)}$ J.A. Coller, ${ }^{(6)}$ M.R. Convery, ${ }^{(29)}$

V. Cook, ${ }^{(38)}$ R.F. Cowan, ${ }^{(19)}$ D.G. Coyne, ${ }^{(35)}$ G. Crawford, ${ }^{(29)}$ C.J.S. Damerell, ${ }^{(27)}$

M.N. Danielson, ${ }^{(8)}$ M. Daoudi, ${ }^{(29)}$ N. de Groot, ${ }^{(4)}$ R. Dell'Orso, ${ }^{(25)}$ P.J. Dervan, ${ }^{(5)}$

R. de Sangro, ${ }^{(12)}$ M. Dima, ${ }^{(10)}$ A. D'Oliveira, ${ }^{(7)}$ D.N. Dong, ${ }^{(19)}$ M. Doser, ${ }^{(29)}$

R. Dubois, ${ }^{(29)}$ B.I. Eisenstein, ${ }^{(13)}$ V. Eschenburg, ${ }^{(18)}$ E. Etzion, ${ }^{(39)}$ S. Fahey, ${ }^{(8)}$ 
The SLD Collaboration

D. Falciai, ${ }^{(12)}$ C. Fan, ${ }^{(8)}$ J.P. Fernandez, ${ }^{(35)}$ M.J. Fero, ${ }^{(19)}$ K. Flood, ${ }^{(17)}$ R. Frey, ${ }^{(22)}$ J. Gifford, ${ }^{(36)}$ T. Gillman, ${ }^{(27)}$ G. Gladding, ${ }^{(13)}$ S. Gonzalez, ${ }^{(19)}$ E.R. Goodman, ${ }^{(8)}$

E.L. Hart, ${ }^{(32)}$ J.L. Harton, ${ }^{(10)}$ A. Hasan, ${ }^{(5)}$ K. Hasuko, ${ }^{(33)}$ S.J. Hedges, ${ }^{(6)}$ S.S. Hertzbach, ${ }^{(17)}$ M.D. Hildreth, ${ }^{(29)}$ J. Huber, ${ }^{(22)}$ M.E. Huffer, ${ }^{(29)}$

E.W. Hughes, ${ }^{(29)}$ X. Huynh, ${ }^{(29)}$ H. Hwang, ${ }^{(22)}$ M. Iwasaki, ${ }^{(22)}$ D.J. Jackson, ${ }^{(27)}$

P. Jacques, ${ }^{(28)}$ J.A. Jaros, ${ }^{(29)}$ Z.Y. Jiang, ${ }^{(29)}$ A.S. Johnson, ${ }^{(29)}$ J.R. Johnson, ${ }^{(39)}$

R.A. Johnson, ${ }^{(7)}$ T. Junk, ${ }^{(29)}$ R. Kajikawa, ${ }^{(21)}$ M. Kalelkar, ${ }^{(28)}$ Y. Kamyshkov, ${ }^{(32)}$

H.J. Kang, ${ }^{(28)}$ I. Karliner, ${ }^{(13)}$ H. Kawahara, ${ }^{(29)}$ Y.D. Kim, ${ }^{(30)}$ M.E. King, ${ }^{(29)}$

R. King, ${ }^{(29)}$ R.R. Kofler, ${ }^{(17)}$ N.M. Krishna, ${ }^{(8)}$ R.S. Kroeger, ${ }^{(18)}$ M. Langston, ${ }^{(22)}$

A. Lath, ${ }^{(19)}$ D.W.G. Leith, ${ }^{(29)}$ V. Lia, ${ }^{(19)}$ C.Lin, ${ }^{(17)}$ M.X. Liu, ${ }^{(40)}$ X. Liu, ${ }^{(35)}$

M. Loreti, ${ }^{(24)}$ A. Lu, ${ }^{(34)}$ H.L. Lynch, ${ }^{(29)}$ J. Ma, ${ }^{(38)}$ G. Mancinelli, ${ }^{(28)}$ S. Manly, ${ }^{(40)}$

G. Mantovani, ${ }^{(25)}$ T.W. Markiewicz, ${ }^{(29)}$ T. Maruyama, ${ }^{(29)}$ H. Masuda, ${ }^{(29)}$

E. Mazzucato, ${ }^{(11)}$ A.K. McKemey, ${ }^{(5)}$ B.T. Meadows, ${ }^{(7)}$ G. Menegatti, ${ }^{(11)}$

R. Messner, ${ }^{(29)}$ P.M. Mockett, ${ }^{(38)}$ K.C. Moffeit, ${ }^{(29)}$ T.B. Moore, ${ }^{(40)}$ M.Morii, ${ }^{(29)}$

D. Muller, ${ }^{(29)}$ V. Murzin, ${ }^{(20)}$ T. Nagamine, ${ }^{(33)}$ S. Narita, ${ }^{(33)}$ U. Nauenberg, ${ }^{(8)}$

H. Neal, ${ }^{(29)}$ M. Nussbaum, ${ }^{(7)}$ N. Oishi, ${ }^{(21)}$ D. Onoprienko, ${ }^{(32)}$ L.S. Osborne, ${ }^{(19)}$

R.S. Panvini, ${ }^{(37)}$ C.H. Park, ${ }^{(31)}$ T.J. Pavel, ${ }^{(29)}$ I. Peruzzi, ${ }^{(12)}$ M. Piccolo, ${ }^{(12)}$

L. Piemontese, ${ }^{(11)}$ K.T. Pitts, ${ }^{(22)}$ R.J. Plano, ${ }^{(28)}$ R. Prepost, ${ }^{(39)}$ C.Y. Prescott, ${ }^{(29)}$

G.D. Punkar, ${ }^{(29)}$ J. Quigley, ${ }^{(19)}$ B.N. Ratcliff, ${ }^{(29)}$ T.W. Reeves, ${ }^{(37)}$ J. Reidy, ${ }^{(18)}$

P.L. Reinertsen, ${ }^{(35)}$ P.E. Rensing, ${ }^{(29)}$ L.S. Rochester, ${ }^{(29)}$ P.C. Rowson, ${ }^{(9)}$

J.J. Russell, ${ }^{(29)}$ O.H. Saxton, ${ }^{(29)}$ T. Schalk, ${ }^{(35)}$ R.H. Schindler, ${ }^{(29)}$ B.A. Schumm, ${ }^{(35)}$

J. Schwiening, ${ }^{(29)}$ S. Sen, ${ }^{(40)}$ V.V. Serbo, ${ }^{(29)}$ M.H. Shaevitz, ${ }^{(9)}$ J.T. Shank, ${ }^{(6)}$

G. Shapiro, ${ }^{(15)}$ D.J. Sherden, ${ }^{(29)}$ K.D. Shmakov, ${ }^{(32)}$ C. Simopoulos, ${ }^{(29)}$

N.B. Sinev, ${ }^{(22)}$ S.R. Smith, ${ }^{(29)}$ M.B. Smy, ${ }^{(10)}$ J.A. Snyder ${ }^{(40)}$ H. Staengle, ${ }^{(10)}$

A. Stahl, ${ }^{(29)}$ P. Stamer, ${ }^{(28)}$ H. Steiner, ${ }^{(15)}$ R. Steiner, ${ }^{(1)}$ M.G. Strauss, ${ }^{(17)}$ D. Su, ${ }^{(29)}$ 
The SLD Collaboration

F. Suekane, ${ }^{(33)}$ A. Sugiyama, ${ }^{(21)}$ S. Suzuki, ${ }^{(21)}$ M. Swartz, ${ }^{(14)}$ A. Szumilo, ${ }^{(38)}$

T. Takahashi, ${ }^{(29)}$ F.E. Taylor, ${ }^{(19)}$ J. Thom, ${ }^{(29)}$ E. Torrence, ${ }^{(19)}$ N.K. Toumbas, ${ }^{(29)}$

T. Usher, ${ }^{(29)}$ C. Vannini, ${ }^{(26)}$ J. Va'vra, ${ }^{(29)}$ E. Vella, ${ }^{(29)}$ J.P. Venuti, ${ }^{(37)}$

R. Verdier, ${ }^{(19)}$ P.G. Verdini, ${ }^{(26)}$ D.L. Wagner, ${ }^{(8)}$ S.R. Wagner, ${ }^{(29)}$ A.P. Waite, ${ }^{(29)}$

S. Walston, ${ }^{(22)}$ S.J. Watts, ${ }^{(5)}$ A.W. Weidemann, ${ }^{(32)}$ E. R. Weiss, ${ }^{(38)}$

J.S. Whitaker, ${ }^{(6)}$ S.L. White, ${ }^{(32)}$ F.J. Wickens, ${ }^{(27)}$ B. Williams, ${ }^{(8)}$ D.C. Williams, ${ }^{(19)}$

S.H. Williams, ${ }^{(29)}$ S. Willocq, ${ }^{(17)}$ R.J. Wilson, ${ }^{(10)}$ W.J. Wisniewski, ${ }^{(29)}$

J. L. Wittlin, ${ }^{(17)}$ M. Woods, ${ }^{(29)}$ G.B. Word, ${ }^{(37)}$ T.R. Wright, ${ }^{(39)}$ J. Wyss, ${ }^{(24)}$

R.K. Yamamoto, ${ }^{(19)}$ J.M. Yamartino, ${ }^{(19)}$ X. Yang, ${ }^{(22)}$ J. Yashima, ${ }^{(33)}$ S.J. Yellin, ${ }^{(34)}$

C.C. Young, ${ }^{(29)}$ H. Yuta, ${ }^{(2)}$ G. Zapalac, ${ }^{(39)}$ R.W. Zdarko, ${ }^{(29)}$ J. Zhou. ${ }^{(22)}$

\section{(The SLD Collaboration)}

(1) Adelphi University, Garden City, New York 11530,

(2) Aomori University, Aomori, 030 Japan,

(3) INFN Sezione di Bologna, I-40126, Bologna, Italy,

(4) University of Bristol, Bristol, U.K.,

${ }^{(5)}$ Brunel University, Uxbridge, Middlesex, UB8 3PH United Kingdom,

(6) Boston University, Boston, Massachusetts 02015,

(7) University of Cincinnati, Cincinnati, Ohio 45201,

(8) University of Colorado, Boulder, Colorado 80309,

(9) Columbia University, New York, New York 10533,

${ }^{(10)}$ Colorado State University, Ft. Collins, Colorado 80523,

(11) INFN Sezione di Ferrara and Universita di Ferrara, I-44100 Ferrara, Italy,

${ }^{(12)}$ INFN Lab. Nazionali di Frascati, I-00044 Frascati, Italy,

${ }^{(13)}$ University of Illinois, Urbana, Illinois 61801,

(14) Johns Hopkins University, Baltimore, Maryland 21218-2686,

${ }^{(15)}$ Lawrence Berkeley Laboratory, University of California, Berkeley, California 94720, 


\section{The SLD Collaboration}

${ }^{(16)}$ Louisiana Technical University, Ruston, Louisiana 71272,

${ }^{(17)}$ University of Massachusetts, Amherst, Massachusetts 01003,

(18) University of Mississippi, University, Mississippi 3867\%,

(19) Massachusetts Institute of Technology, Cambridge, Massachusetts 02139,

${ }^{(20)}$ Institute of Nuclear Physics, Moscow State University, 119899, Moscow Russia,

(21) Nagoya University, Chikusa-ku, Nagoya, 464 Japan,

${ }^{(22)}$ University of Oregon, Eugene, Oregon 97403,

(23) Oxford University, Oxford, OX1 3RH, United Kingdom,

${ }^{(24)}$ INFN Sezione di Padova and Universita di Padova I-35100, Padova, Italy,

${ }^{(25)}$ INFN Sezione di Perugia and Universita di Perugia, I-06100 Perugia, Italy,

(26) INFN Sezione di Pisa and Universita di Pisa, I-56010 Pisa, Italy,

${ }^{(27)}$ Rutherford Appleton Laboratory, Chilton, Didcot, Oxon OX11 OQX United

Kingdom,

${ }^{(28)}$ Rutgers University, Piscataway, New Jersey 0885.5,

(29) Stanford Linear Accelerator Center, Stanford University, Stanford, California

$$
94309
$$

(30) Sogang University, Seoul, Korea,

(31) Soongsil University, Seoul, Korea 156-743,

${ }^{(32)}$ University of Tennessee, Knoxville, Tennessee 37996,

(33) Tohoku University, Sendai 980, Japan,

(34) University of California at Santa Barbara, Santa Barbara, California 93106,

(35) University of California at Santa Cruz, Santa Cruz, California 95064,

${ }^{(36)}$ University of Victoria, Victoria, British Columbia, Canada V8W 3P6,

(37) Vanderbilt University, Nashville, Tennessee 37235,

(38) University of Washington, Seattle, Washington 98105,

${ }^{(39)}$ University of Wisconsin, Madison, Wisconsin 53706,

${ }^{(40)}$ Yale University, New Haven, Connecticut 06511. 


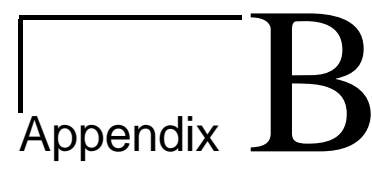

Backup Plots Associated with the Main<smiles>[Al+2]</smiles>

This appendix includes several plots which serve as backup for the main text. 


\section{Backup Plots Associated with the Main Text}
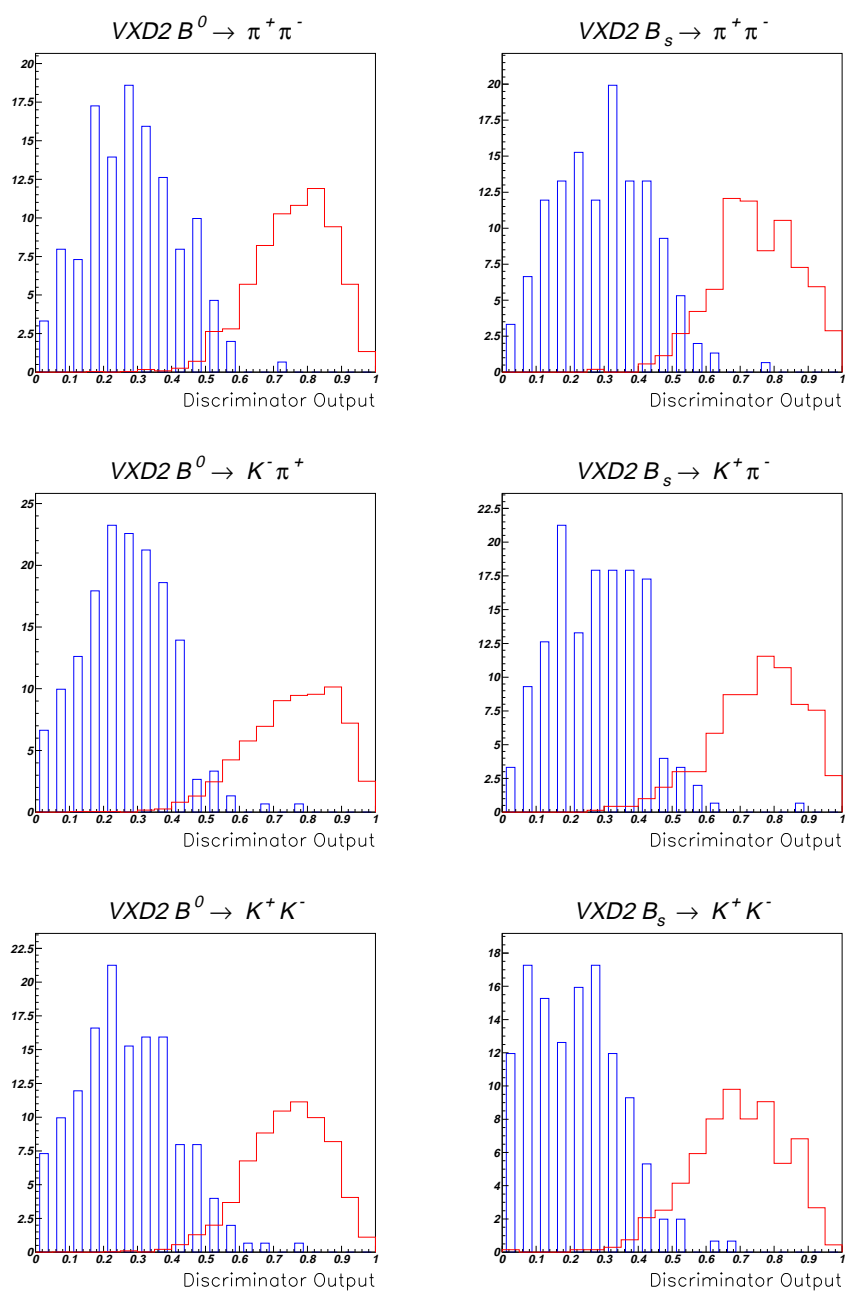

Figure B.1: Shown are the discriminator outputs for VXD2 running period and the $B \rightarrow$ $P P$ modes. The bar chart histograms are the background $\mathrm{MC}$ events and the solid histograms are the signal MC events. The area of the background events corresponds to the number of background events to be seen in the VXD2 data. The area of the signal events is the number of signal events that will be seen if there is 10 events totally produced at the SLD during the VXD2 period. 

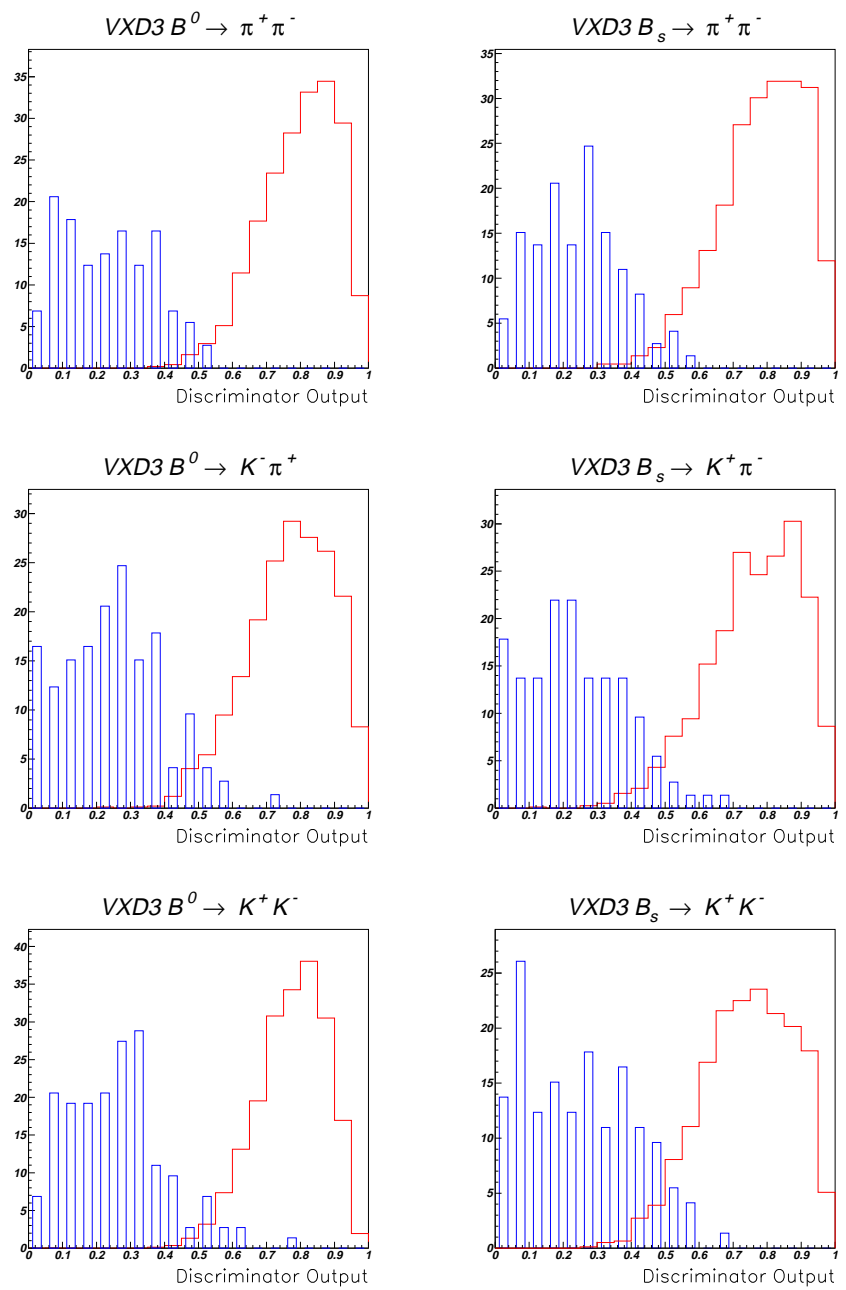

Figure B.2: Shown are the discriminator outputs for VXD3 running period and the $B \rightarrow$ PP modes. The bar chart histograms are the background $\mathrm{MC}$ events and the solid histograms are the signal MC events. The area of the background events corresponds to the number of background events to be seen in the VXD3 data. The area of the signal events is the number of signal events that will be seen if there is 25 events totally produced at the SLD during the VXD3 period. 


\section{Backup Plots Associated with the Main Text}
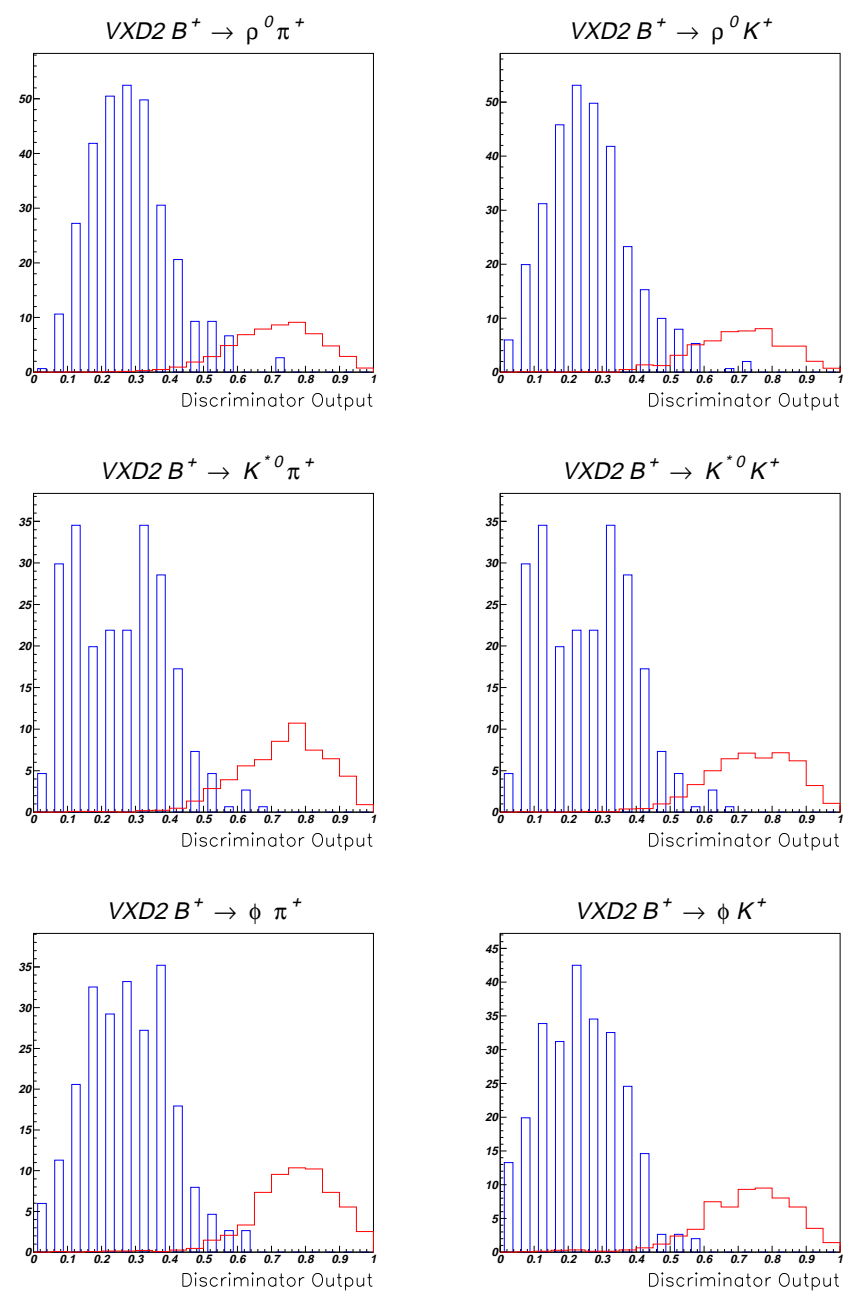

Figure B.3: Shown are the discriminator outputs for VXD2 running period and the $B \rightarrow$ $P V$ modes. The bar chart histograms are the background $\mathrm{MC}$ events and the solid histograms are the signal MC events. The area of the background events corresponds to the number of background events to be seen in the VXD2 data. The area of the signal events is the number of signal events that will be seen if there is 10 events totally produced at the SLD during the VXD2 period. 

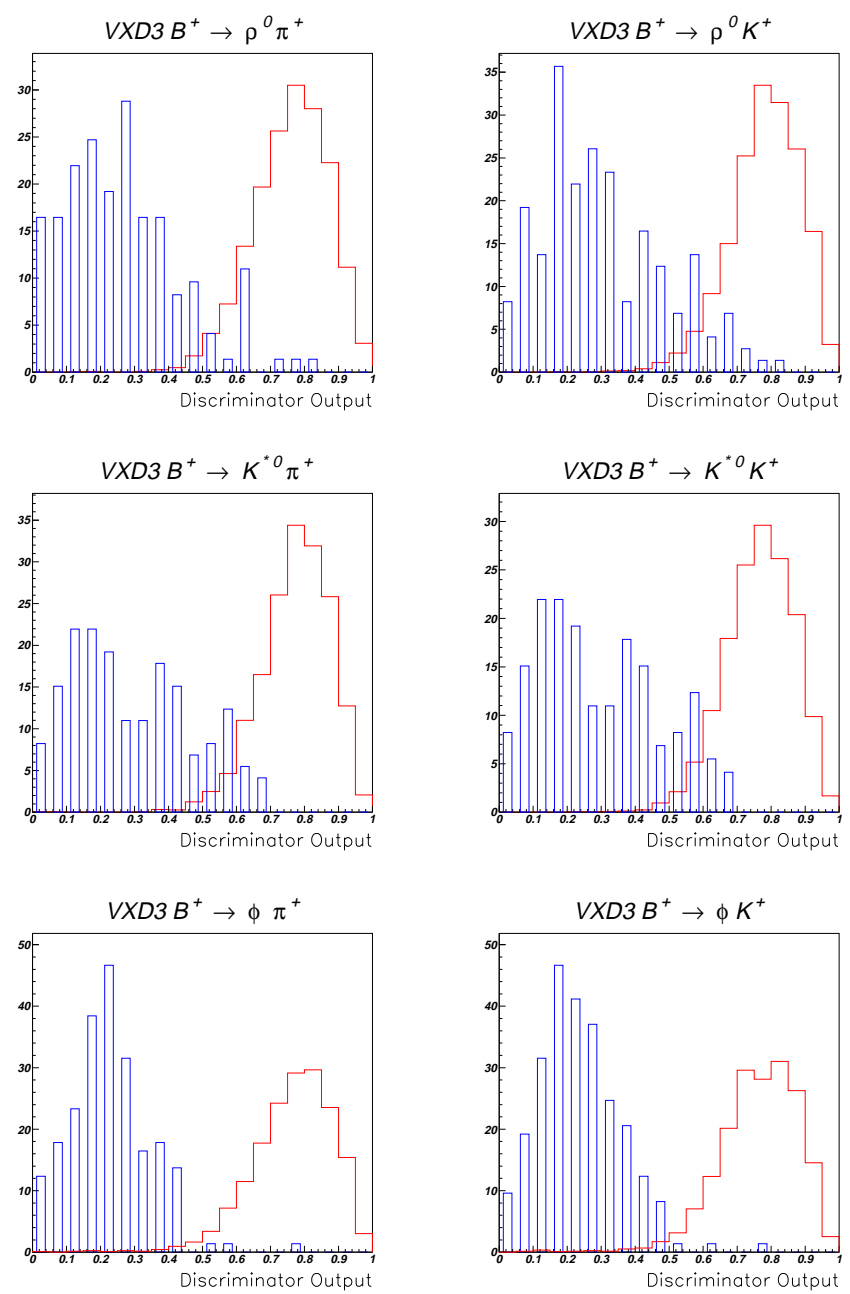

Figure B.4: Shown are the discriminator outputs for VXD3 running period and the $B \rightarrow$ $P V$ modes. The bar chart histograms are the background $\mathrm{MC}$ events and the solid histograms are the signal MC events. The area of the background events corresponds to the number of background events to be seen in the VXD3 data. The area of the signal events is the number of signal events that will be seen if there is 25 events totally produced at the SLD during the VXD3 period. 


\section{Backup Plots Associated with the Main Text}
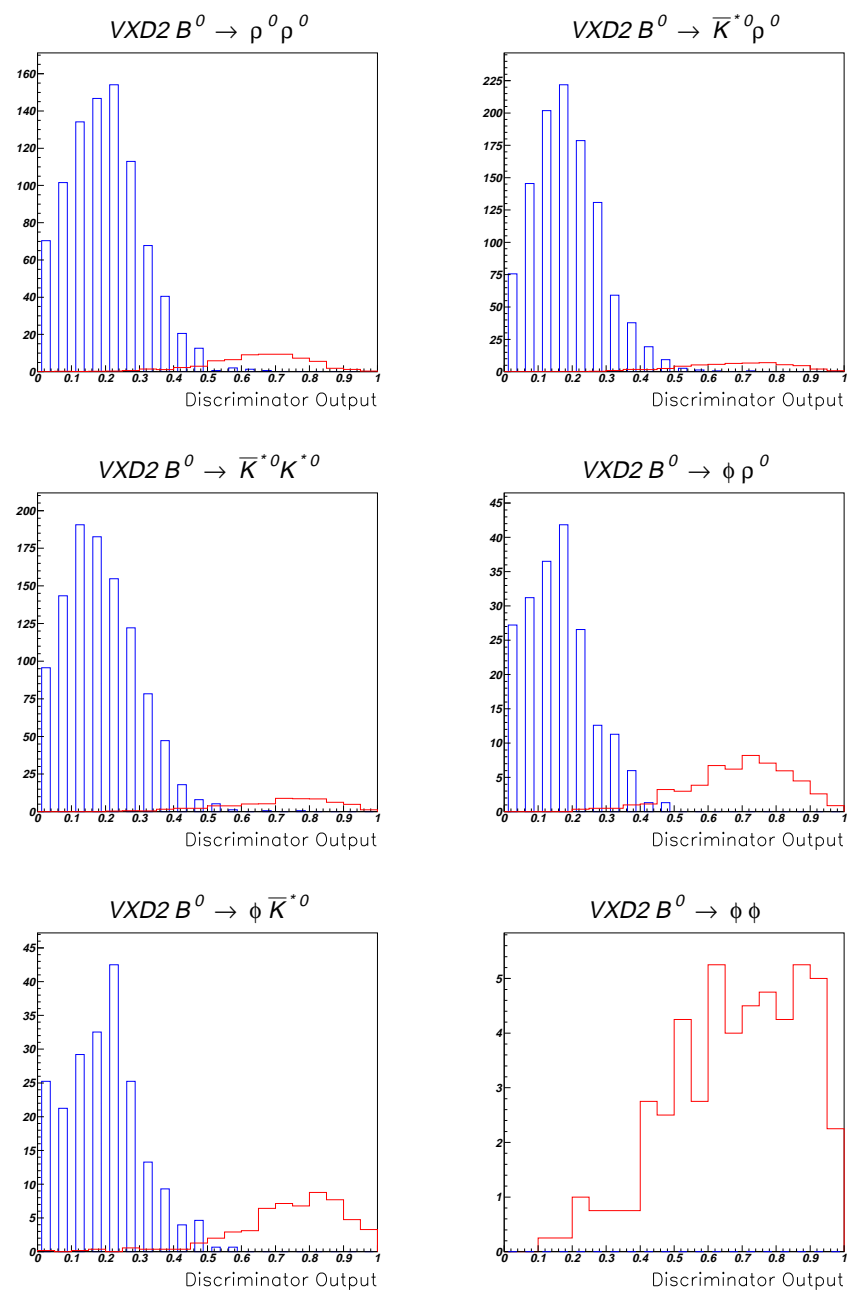

Figure B.5: Shown are the discriminator outputs for VXD2 running period and the $B^{0} \rightarrow$ $V V$ modes. The bar chart histograms are the background $\mathrm{MC}$ events and the solid histograms are the signal MC events. The area of the background events corresponds to the number of background events to be seen in the VXD2 data. The area of the signal events is the number of signal events that will be seen if there is 10 events totally produced at the SLD during the VXD2 period. 

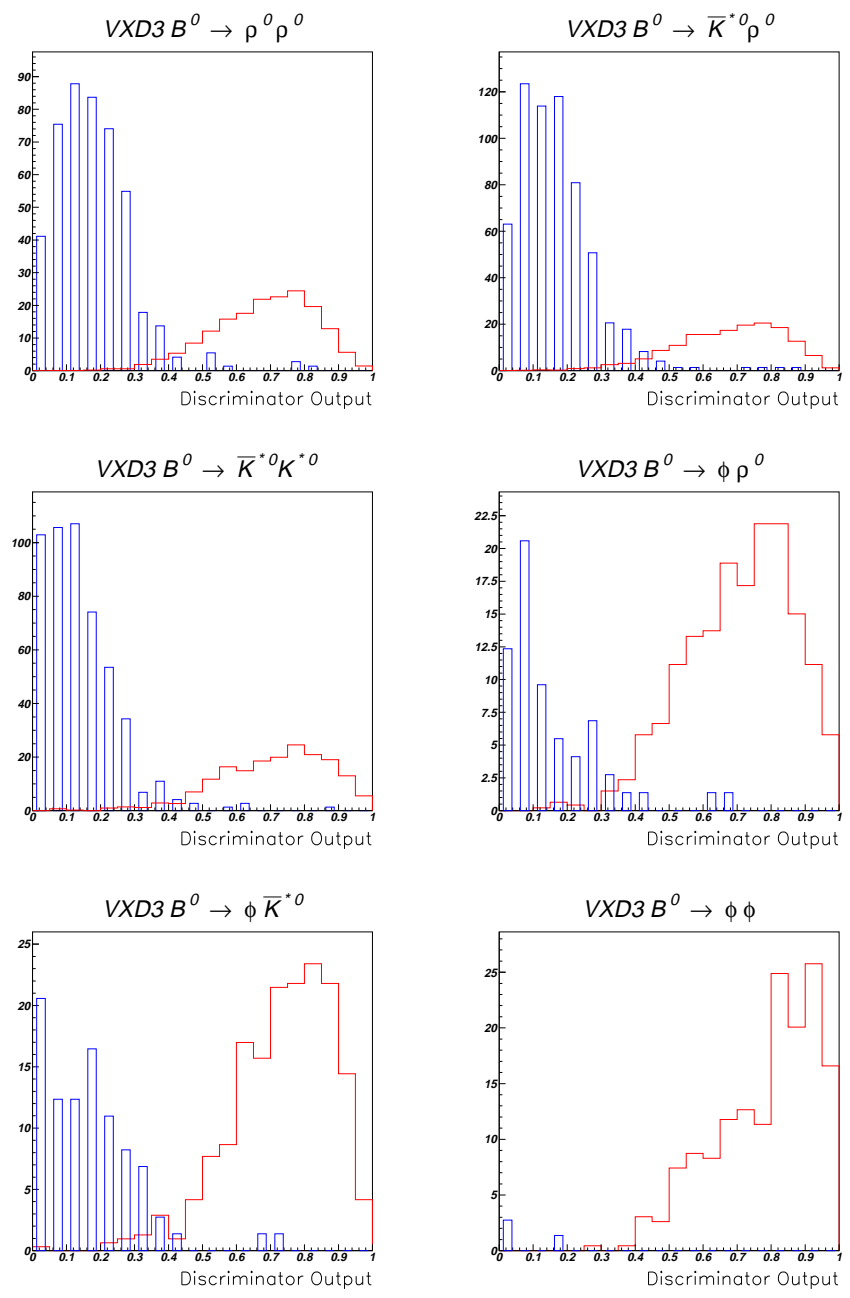

Figure B.6: Shown are the discriminator outputs for VXD3 running period and the $B^{0} \rightarrow$ $V V$ modes. The bar chart histograms are the background $\mathrm{MC}$ events and the solid histograms are the signal MC events. The area of the background events corresponds to the number of background events to be seen in the VXD3 data. The area of the signal events is the number of signal events that will be seen if there is 25 events totally produced at the SLD during the VXD3 period. 


\section{Backup Plots Associated with the Main Text}
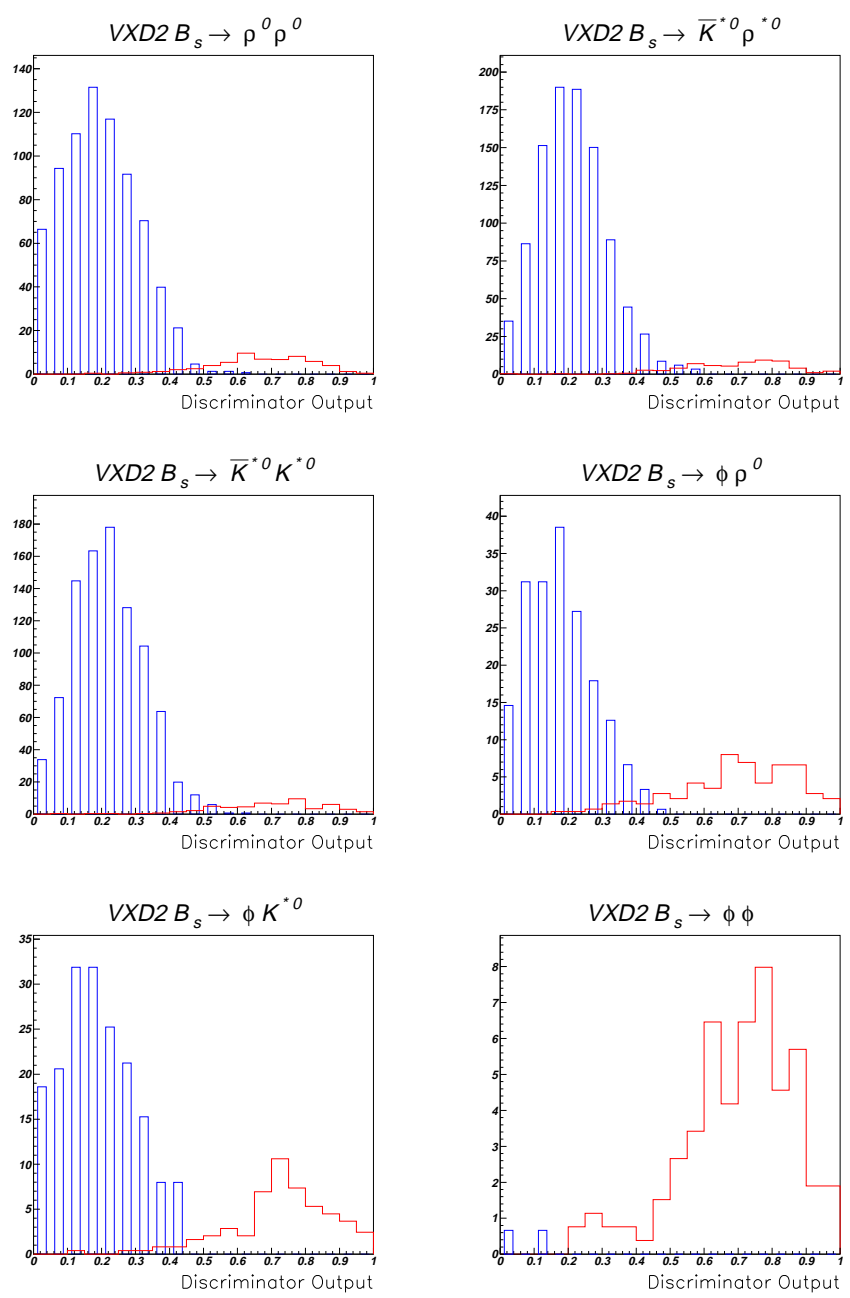

Figure B.7: Shown are the discriminator outputs for VXD2 running period and the $B_{s} \rightarrow$ $V V$ modes. The bar chart histograms are the background $\mathrm{MC}$ events and the solid histograms are the signal MC events. The area of the background events corresponds to the number of background events to be seen in the VXD2 data. The area of the signal events is the number of signal events that will be seen if there is 10 events totally produced at the SLD during the VXD2 period. 

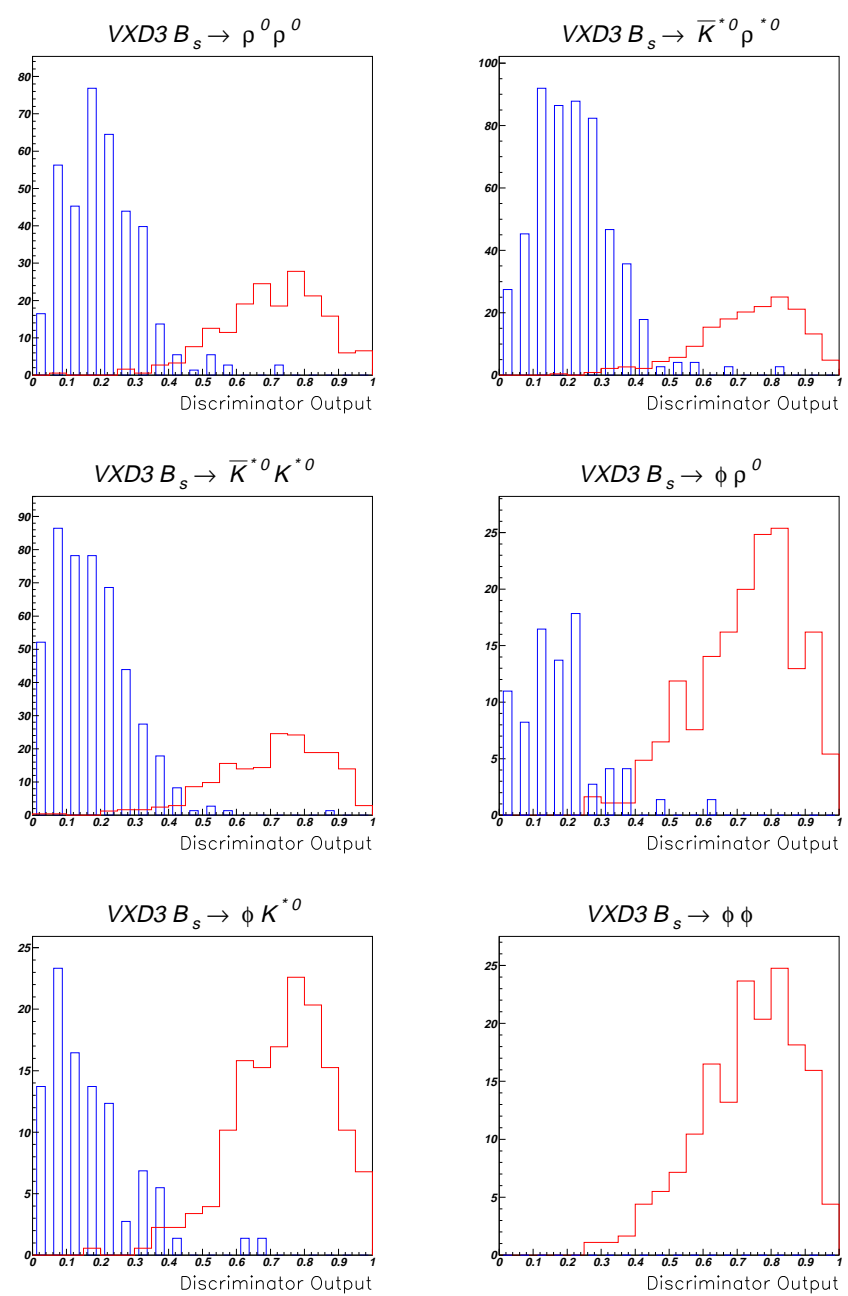

Figure B.8: Shown are the discriminator outputs for VXD3 running period and the $B_{s} \rightarrow$ $V V$ modes. The bar chart histograms are the background $\mathrm{MC}$ events and the solid histograms are the signal MC events. The area of the background events corresponds to the number of background events to be seen in the VXD3 data. The area of the signal events is the number of signal events that will be seen if there is 25 events totally produced at the SLD during the VXD3 period. 


\section{Backup Plots Associated with the Main Text}
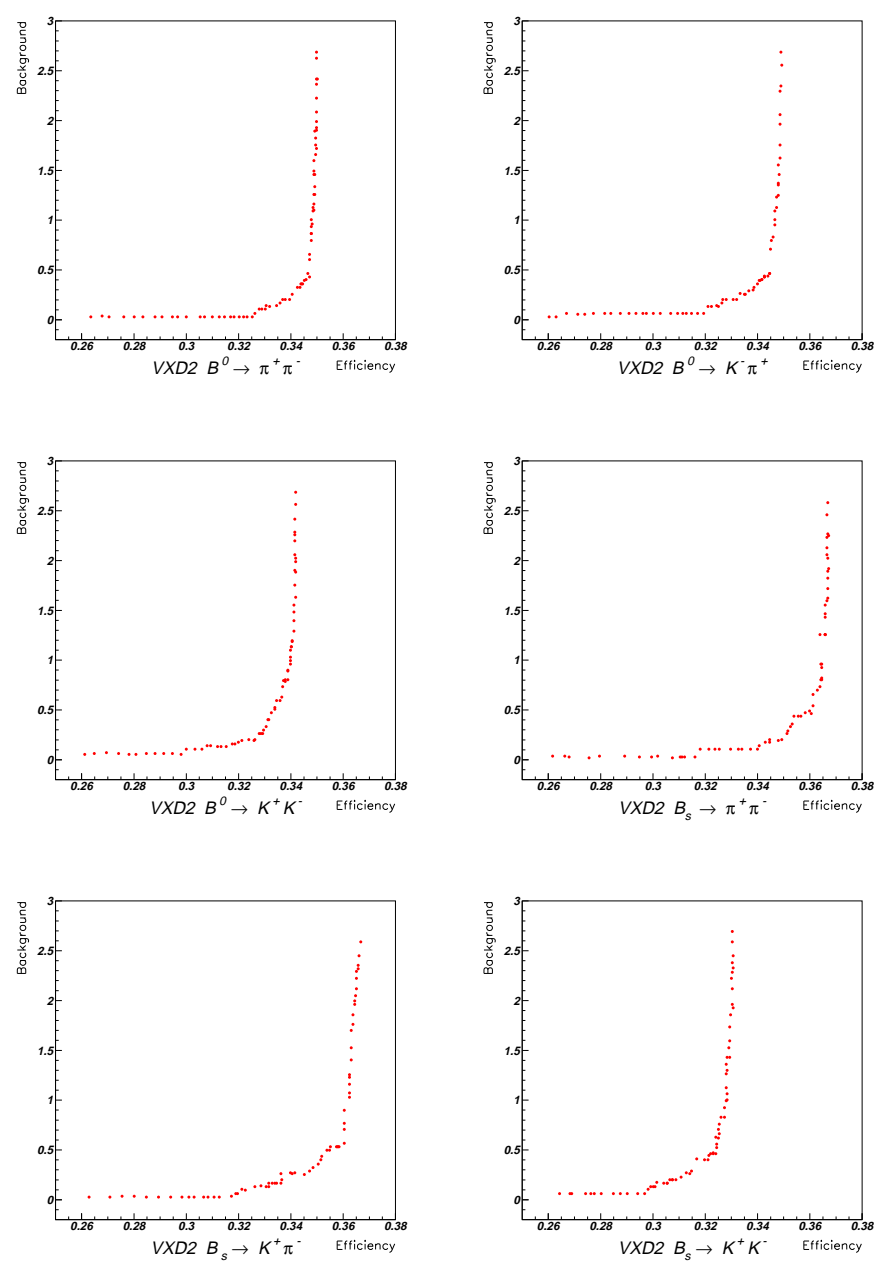

Figure B.9: Shown are the background versus the efficiency for the $B \rightarrow P P$ modes in the VXD2 period. 


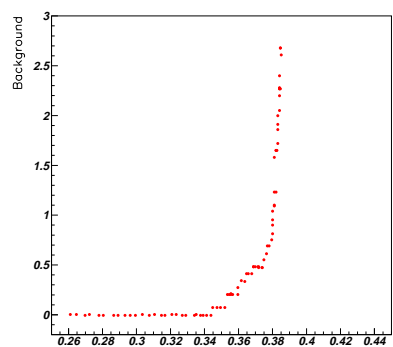

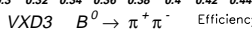
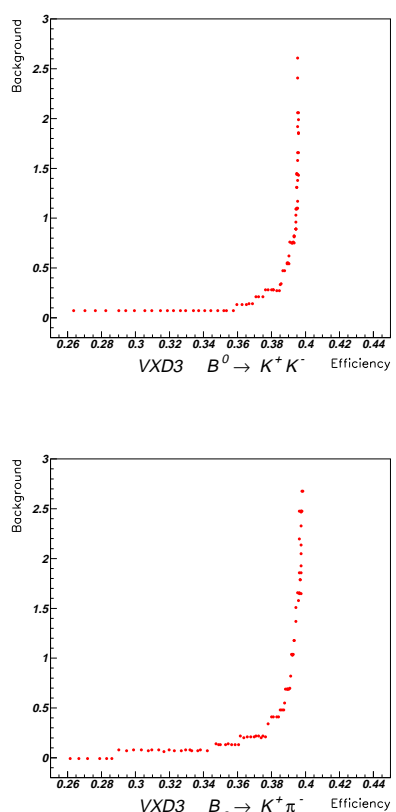

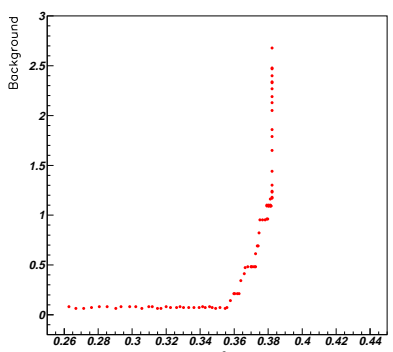

$\begin{array}{lllll}V X D 3 & B^{0} \rightarrow K^{-} \pi^{+} & \text {Éficiency }\end{array}$
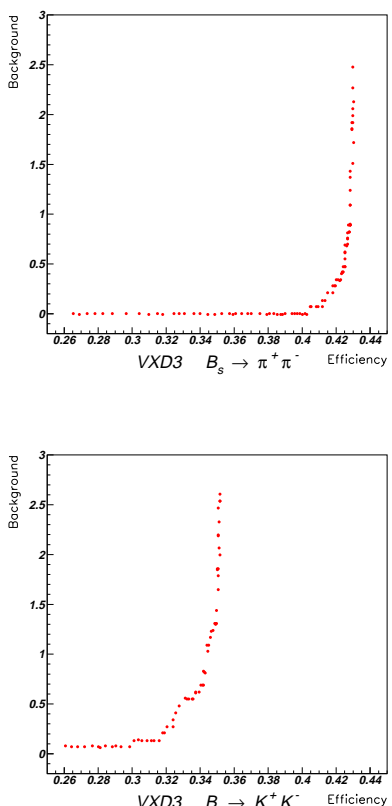

Figure B.10: Shown are the background versus the efficiency for the $B \rightarrow P P$ modes in the VXD3 period. 


\section{Backup Plots Associated with the Main Text}
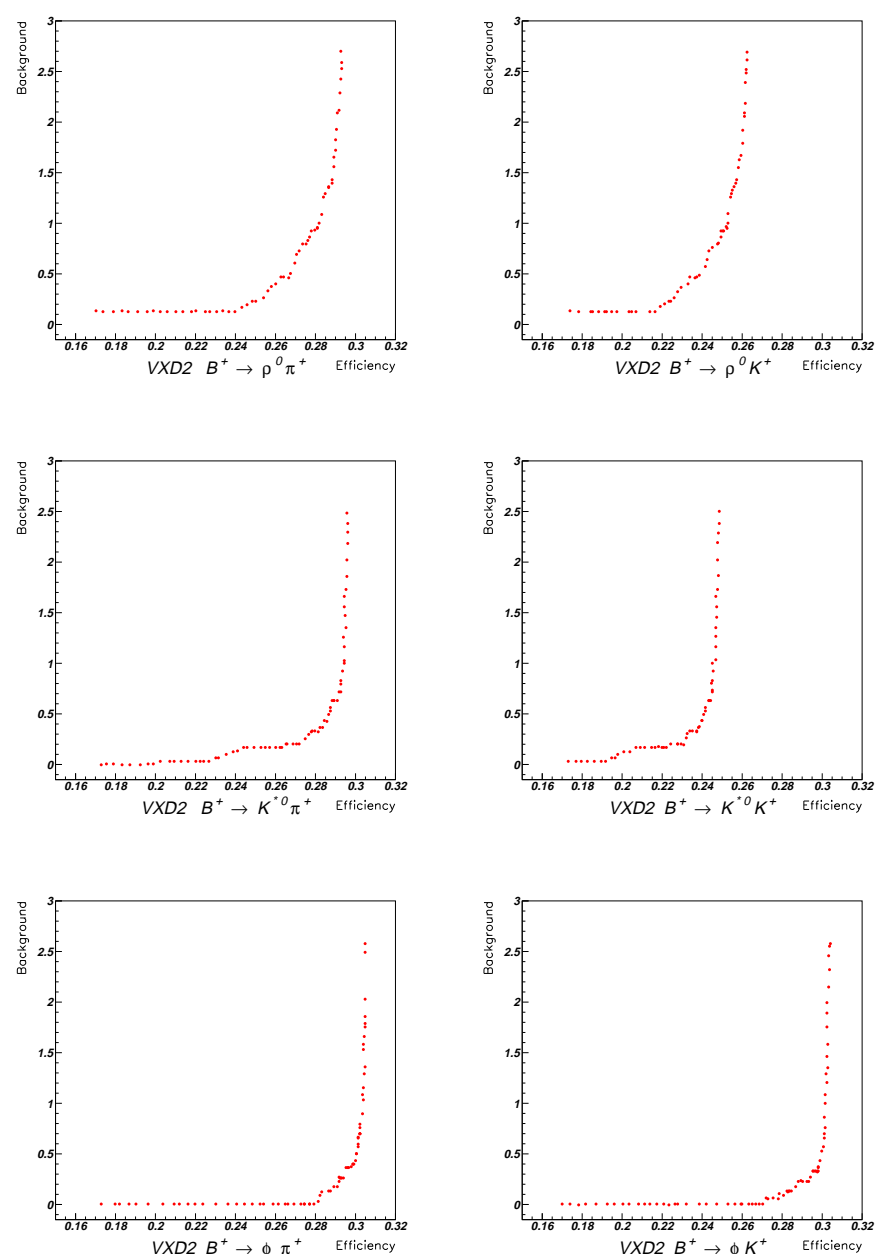

Figure B.11: Shown are the background versus the efficiency for the $B \rightarrow P V$ modes in the VXD2 period. 

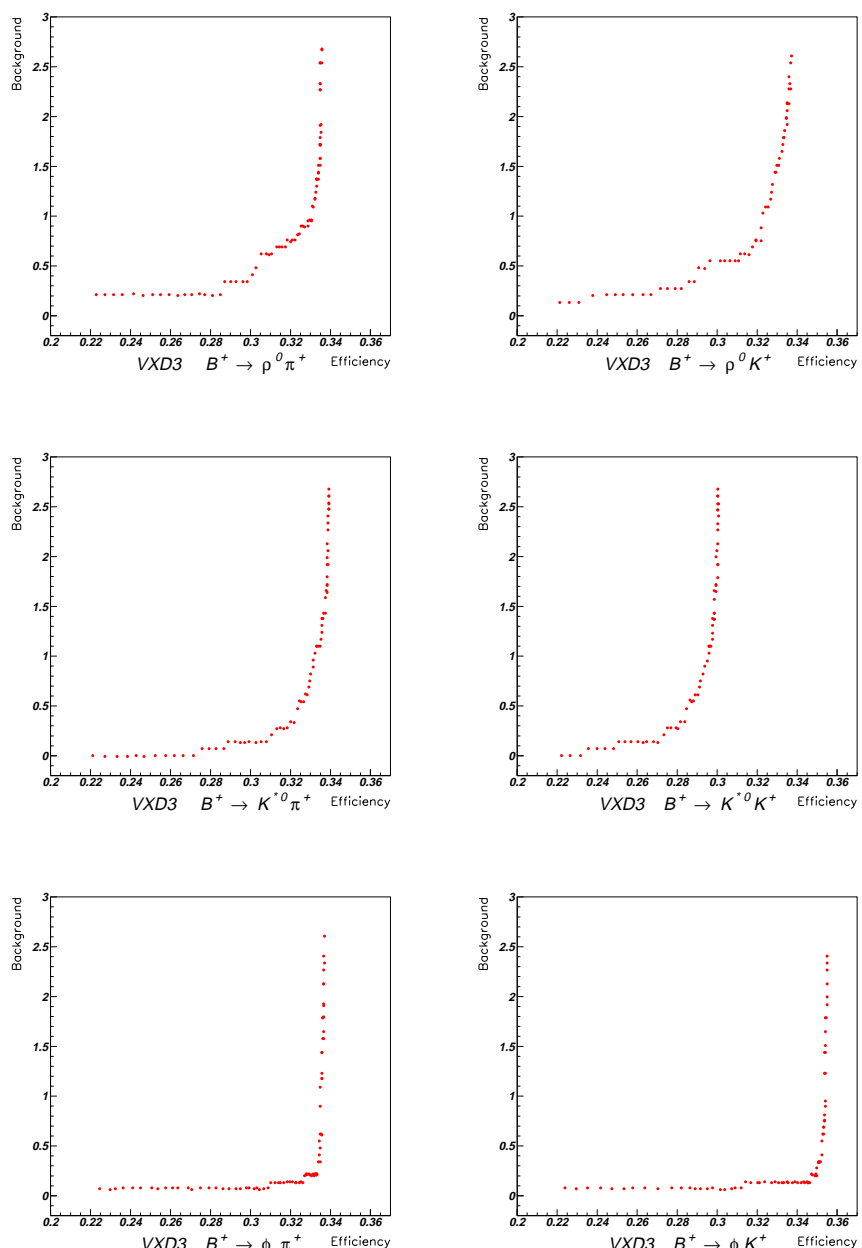

Figure B.12: Shown are the background versus the efficiency for the $B \rightarrow P V$ modes in the VXD3 period. 


\section{Backup Plots Associated with the Main Text}
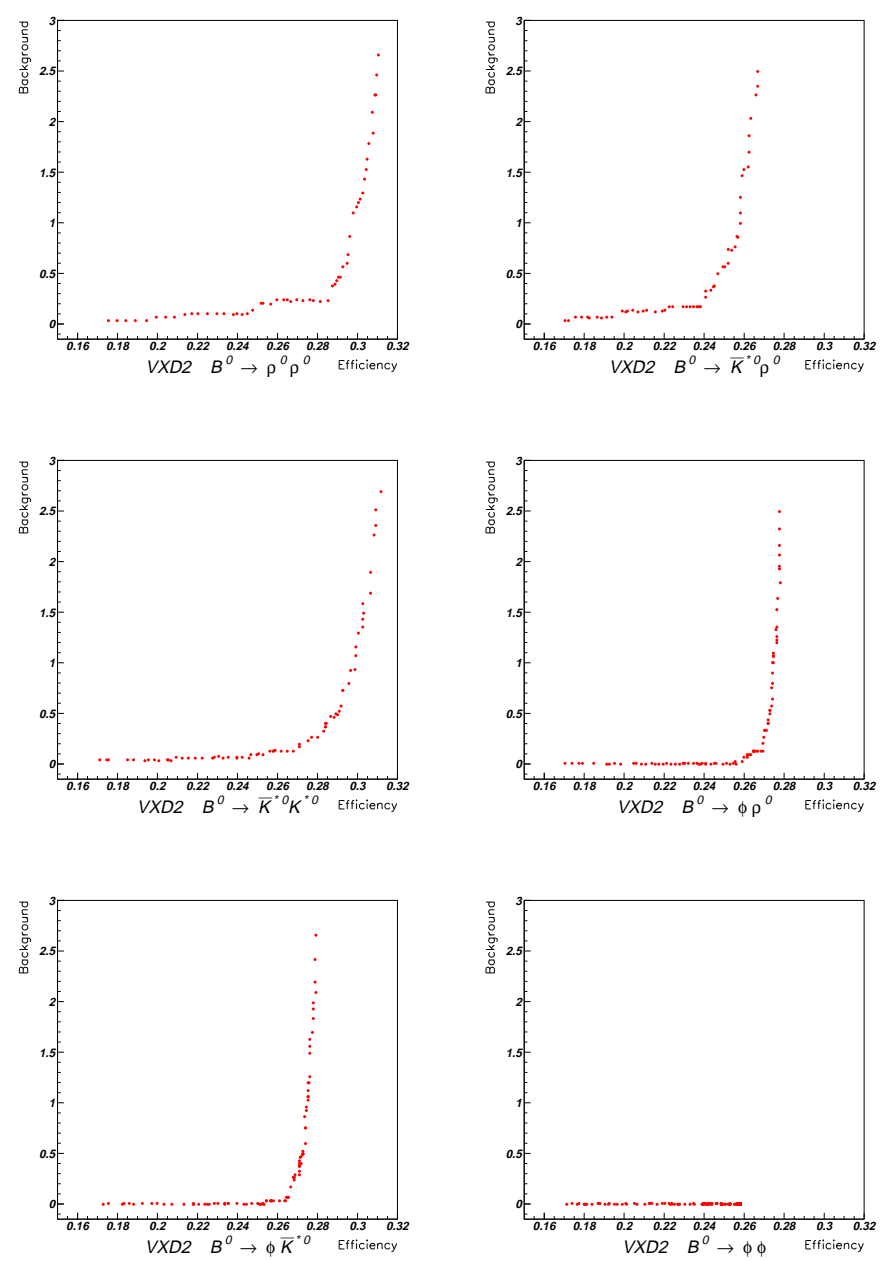

Figure B.13: Shown are the background versus the efficiency for the $B^{0} \rightarrow V V$ modes in the VXD2 period. 

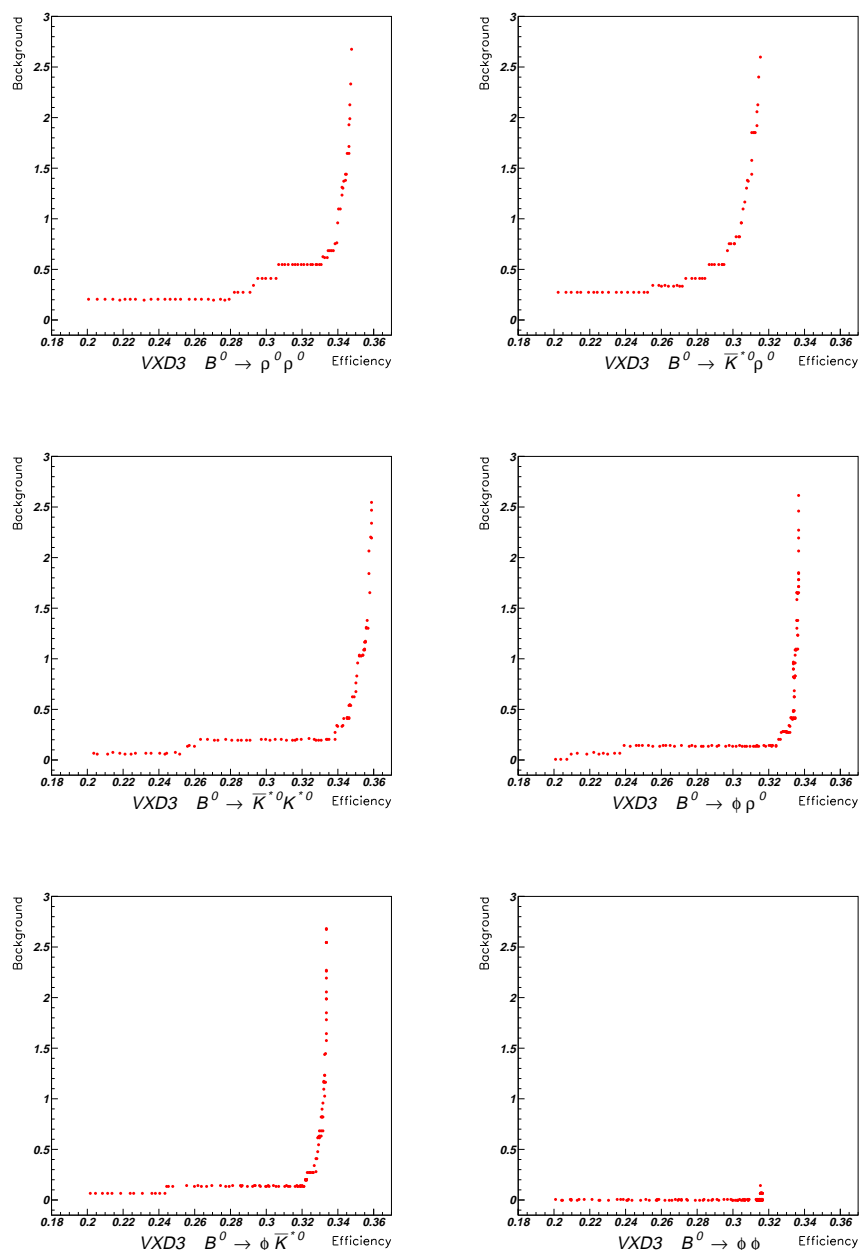

Figure B.14: Shown are the background versus the efficiency for the $B^{0} \rightarrow V V$ modes in the VXD3 period. 


\section{Backup Plots Associated with the Main Text}
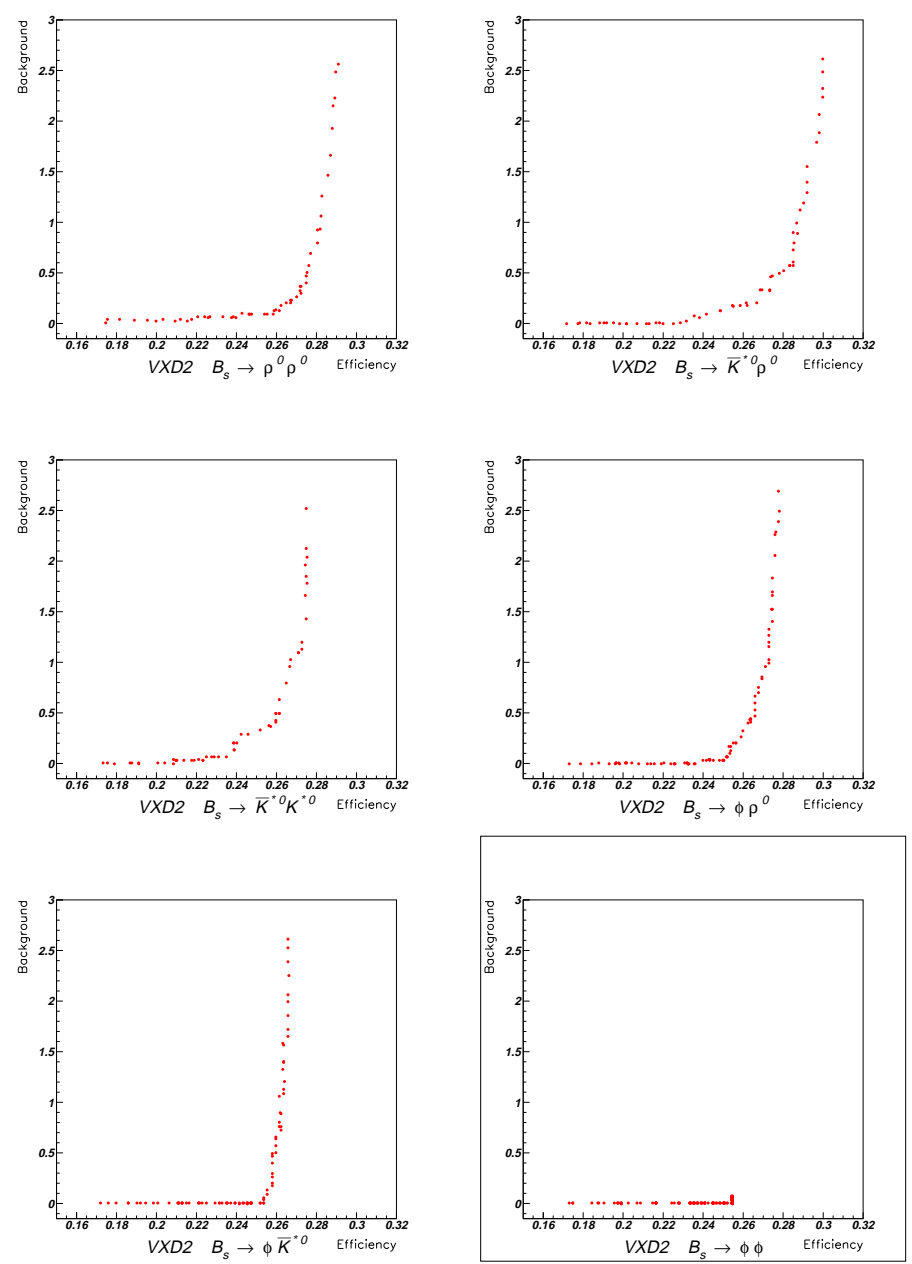

Figure B.15: Shown are the background versus the efficiency for the $B_{s} \rightarrow V V$ modes in the VXD2 period. 


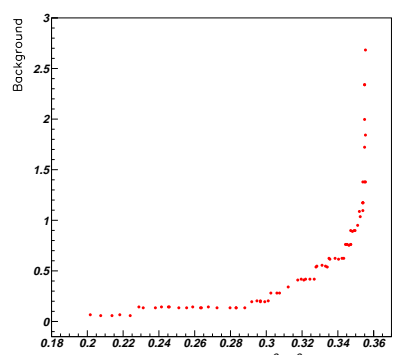

$V X D 3 \quad B_{s} \rightarrow \rho^{0} \rho^{0} \quad$ Eticierc
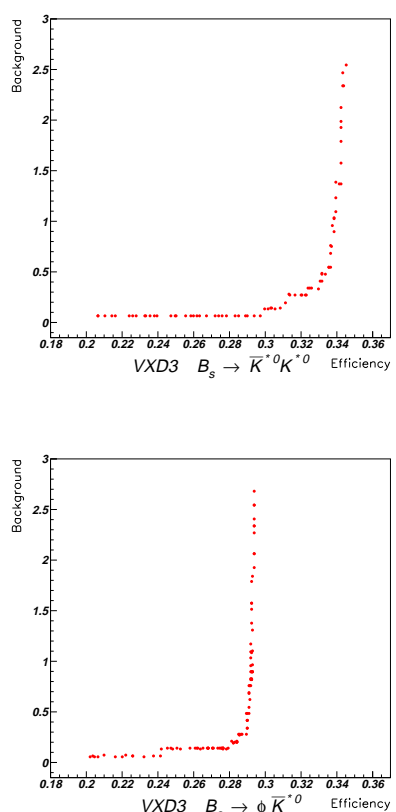
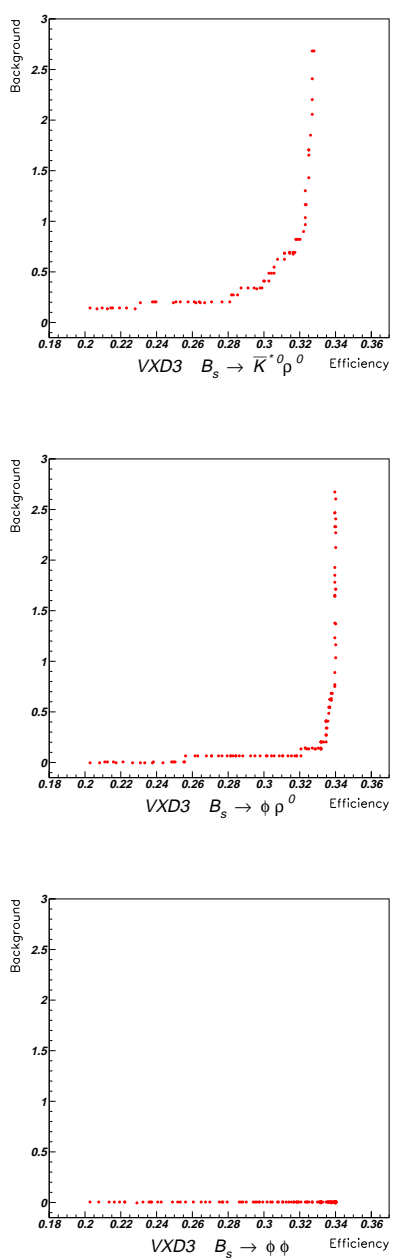

Figure B.16: Shown are the background versus the efficiency for the $B_{s} \rightarrow V V$ modes in the VXD3 period. 


\section{Backup Plots Associated with the Main Text}
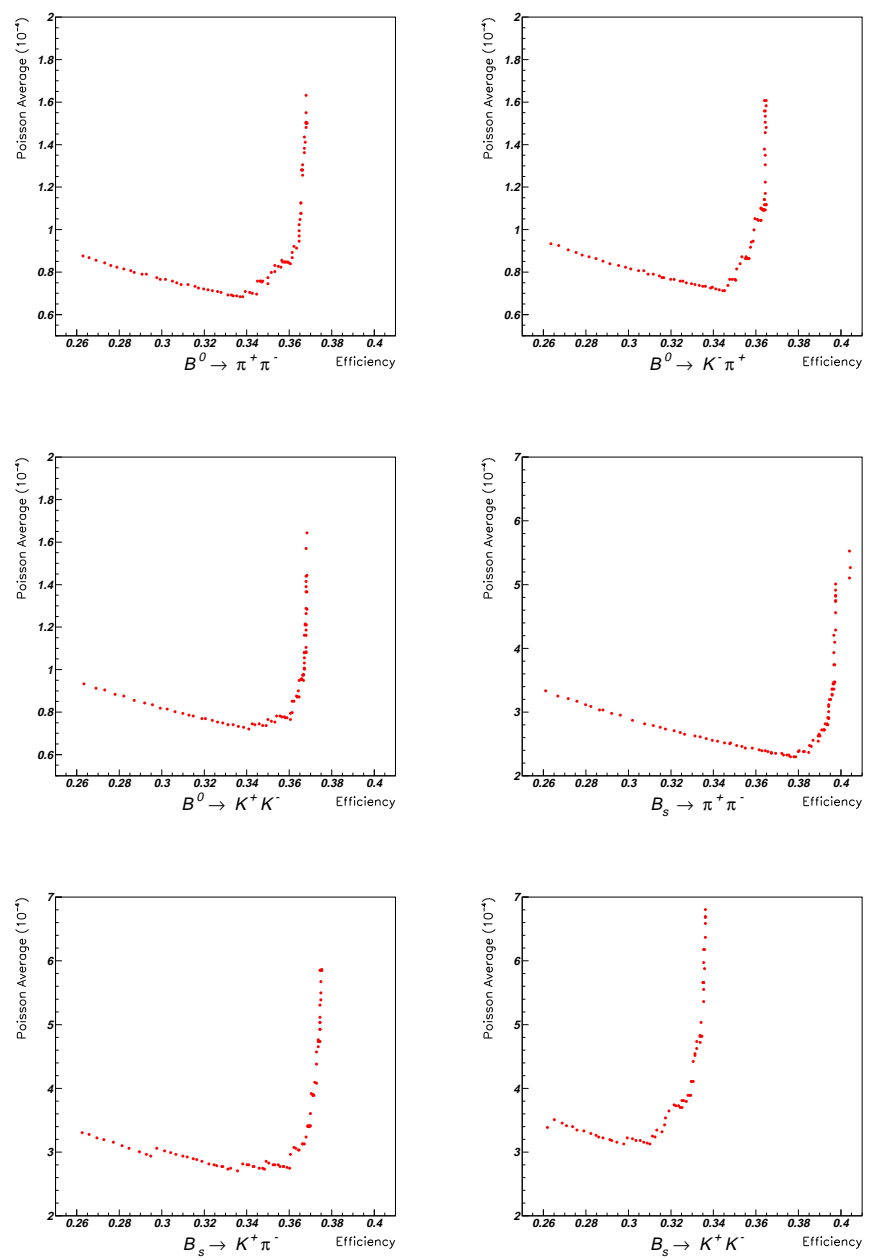

Figure B.17: Shown are the combined Poisson average versus the efficiency for the $B \rightarrow$ PP modes. 

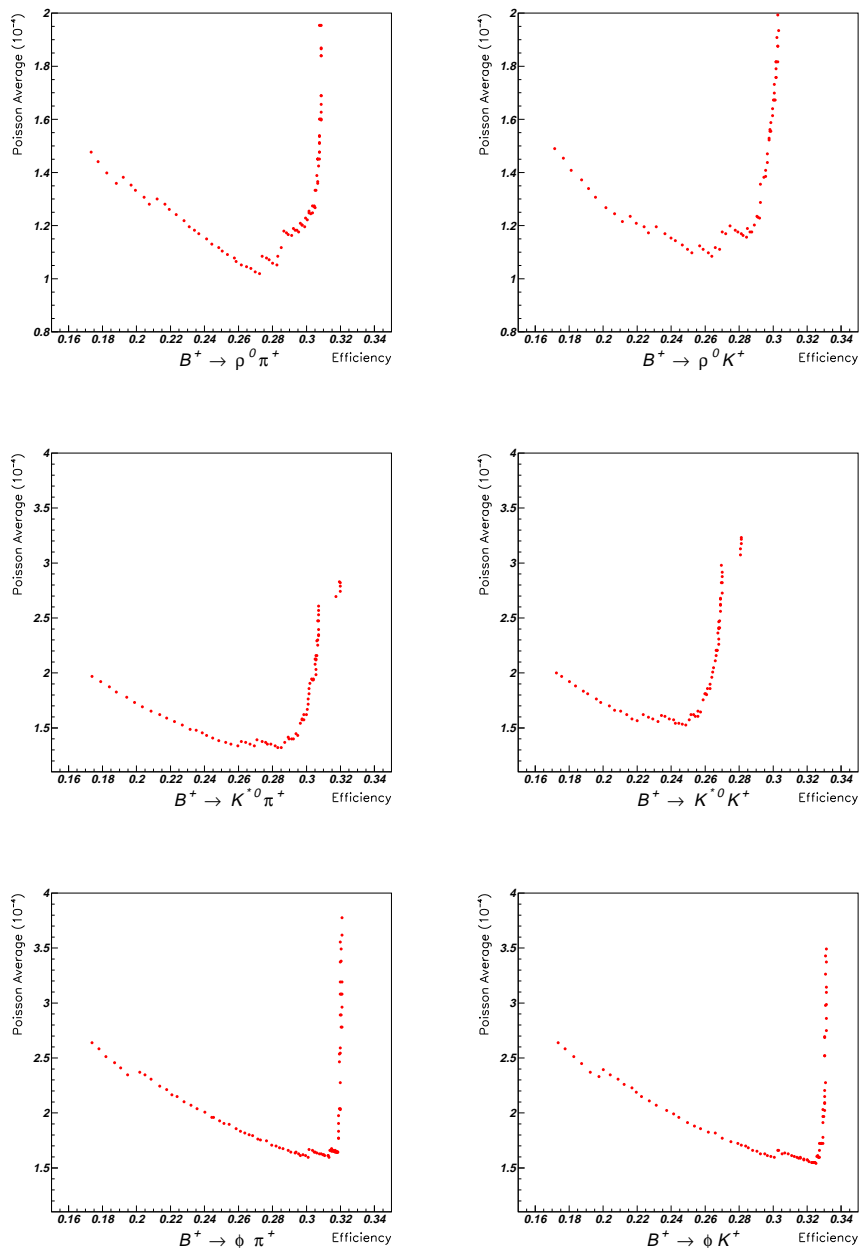

Figure B.18: Shown are the combined Poisson average versus the efficiency for the $B \rightarrow$ $P V$ modes. 


\section{Backup Plots Associated with the Main Text}
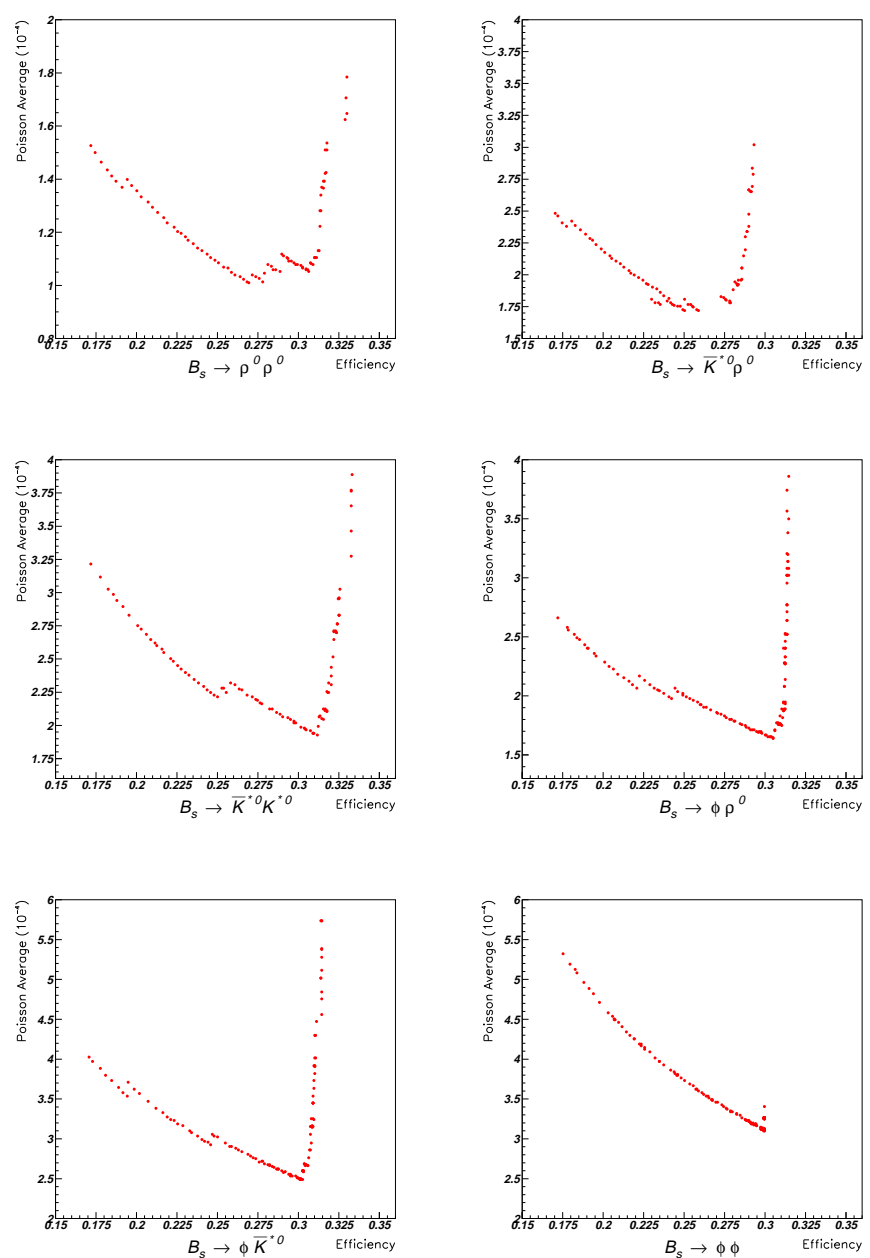

Figure B.19: Shown are the combined Poisson average versus the efficiency for the $B^{0} \rightarrow$ $V V$ modes. 

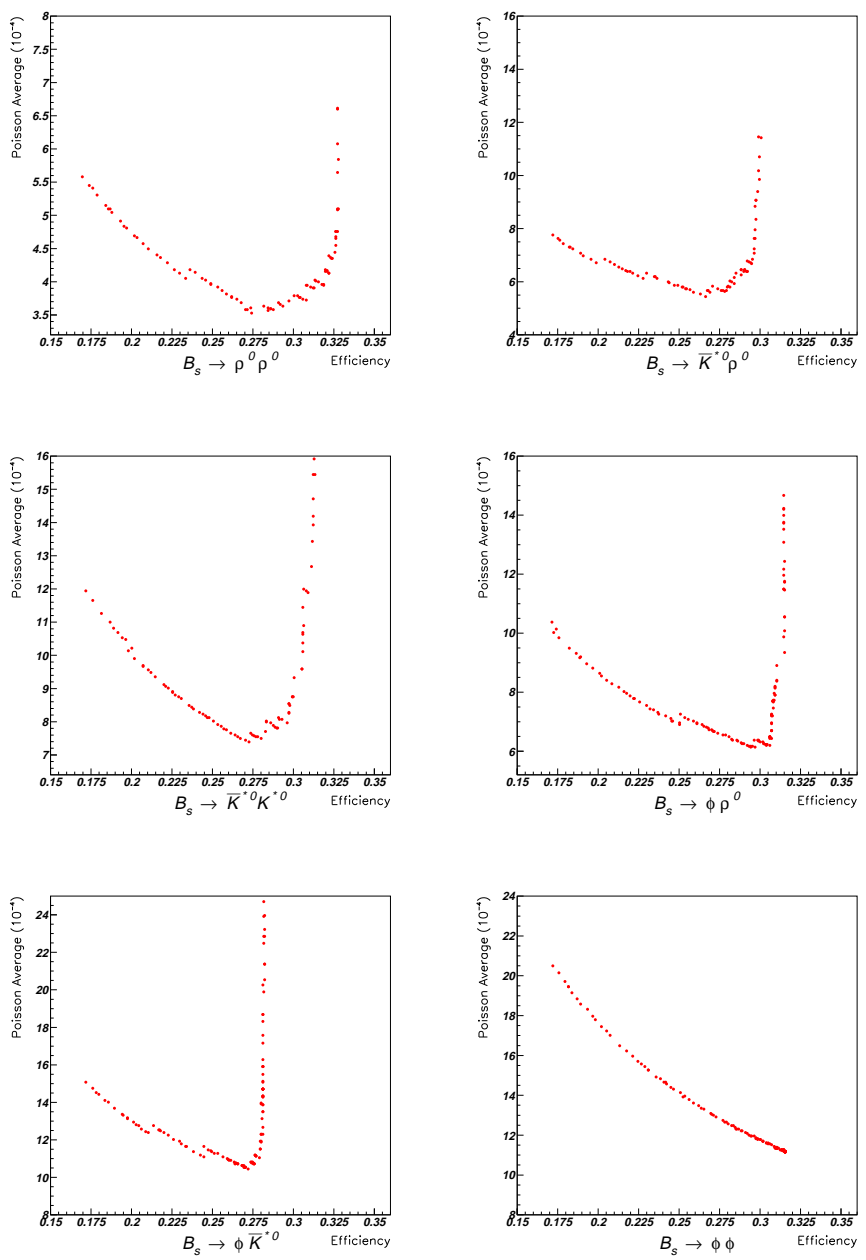

Figure B.20: Shown are the combined Poisson average versus the efficiency for the $B_{s} \rightarrow$ $V V$ modes. 


\section{Backup Plots Associated with the Main Text}
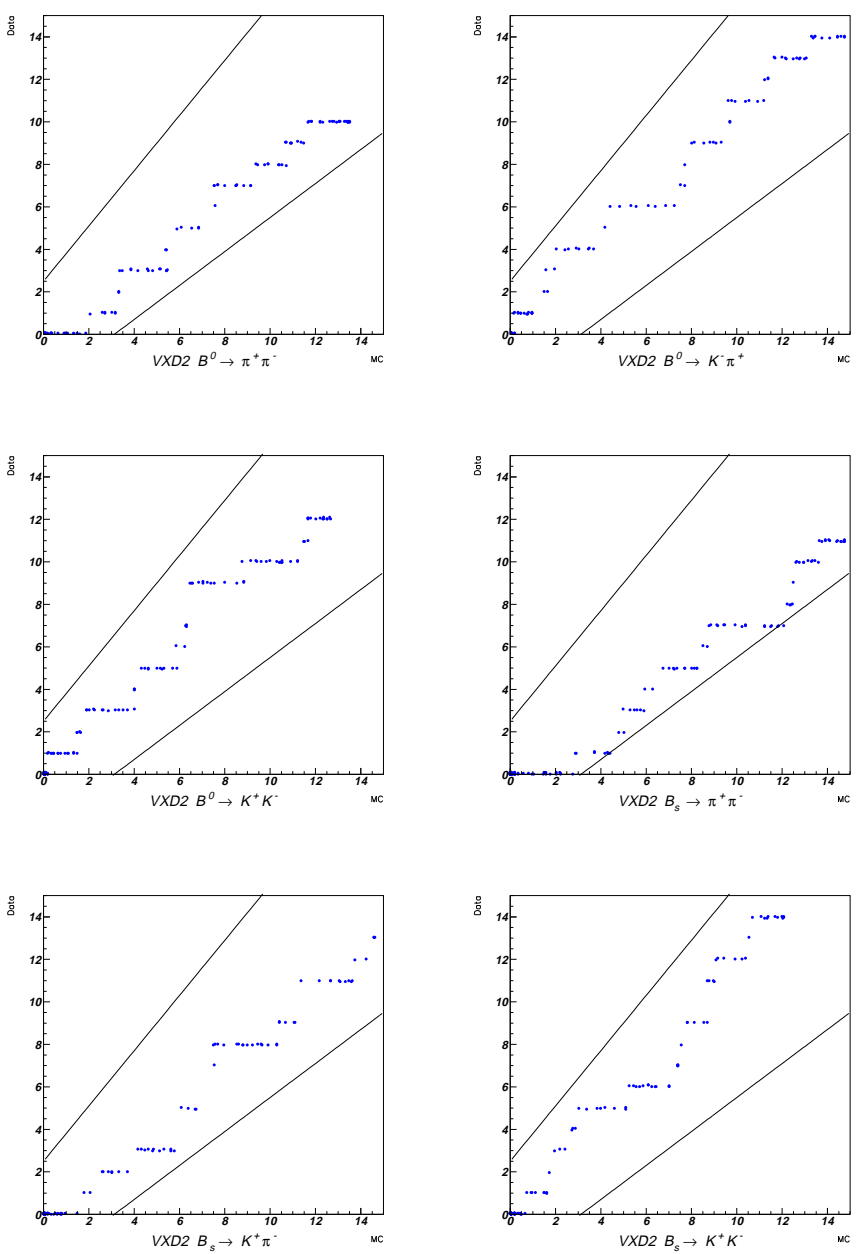

Figure B.21: Shown are the MC expected background level plotted versus the actual number of events seen in the data for VXD2 $B \rightarrow P P$. The lines indicates an approximately $90 \%$ confidence interval. 

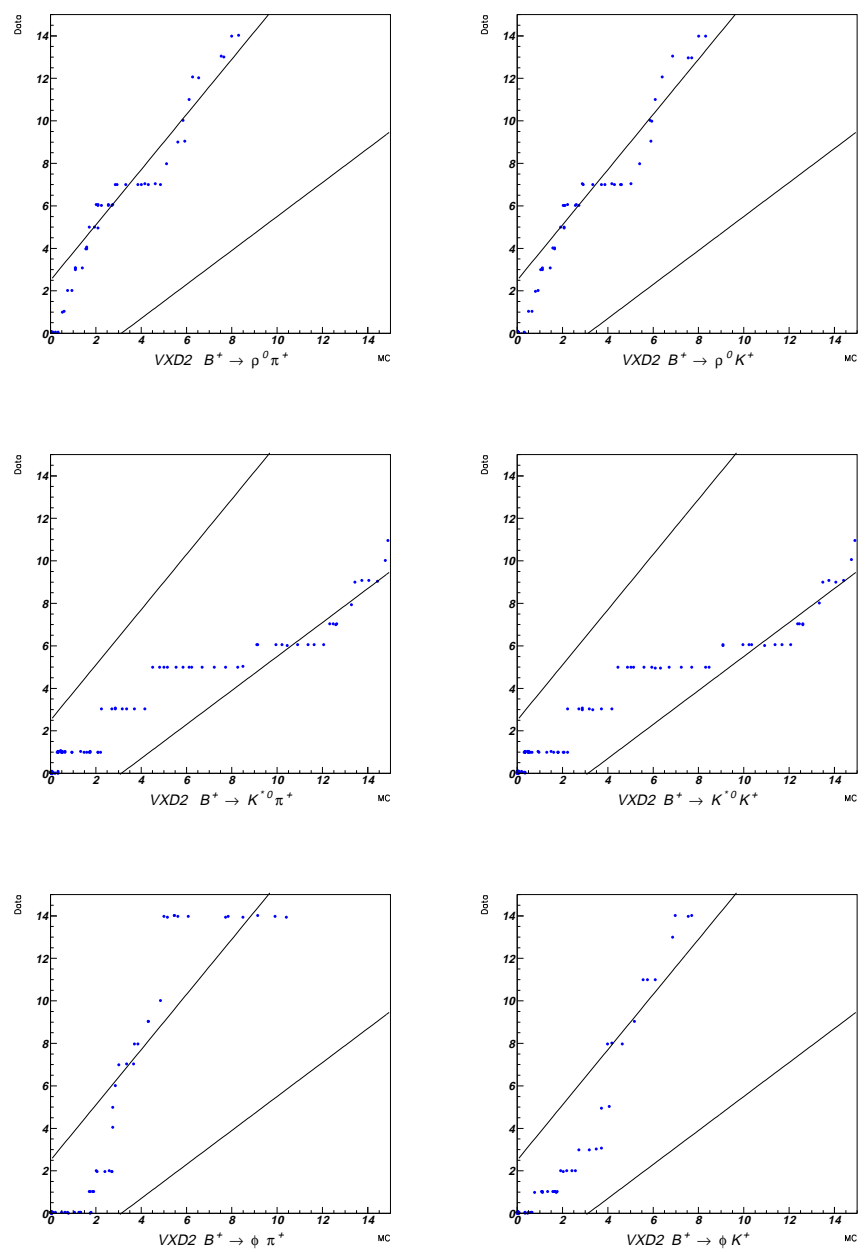

Figure B.22: Shown are the MC expected background level plotted versus the actual number of events seen in the data for VXD2 $B \rightarrow P V$. The lines indicates an approximately $90 \%$ confidence interval. 


\section{Backup Plots Associated with the Main Text}
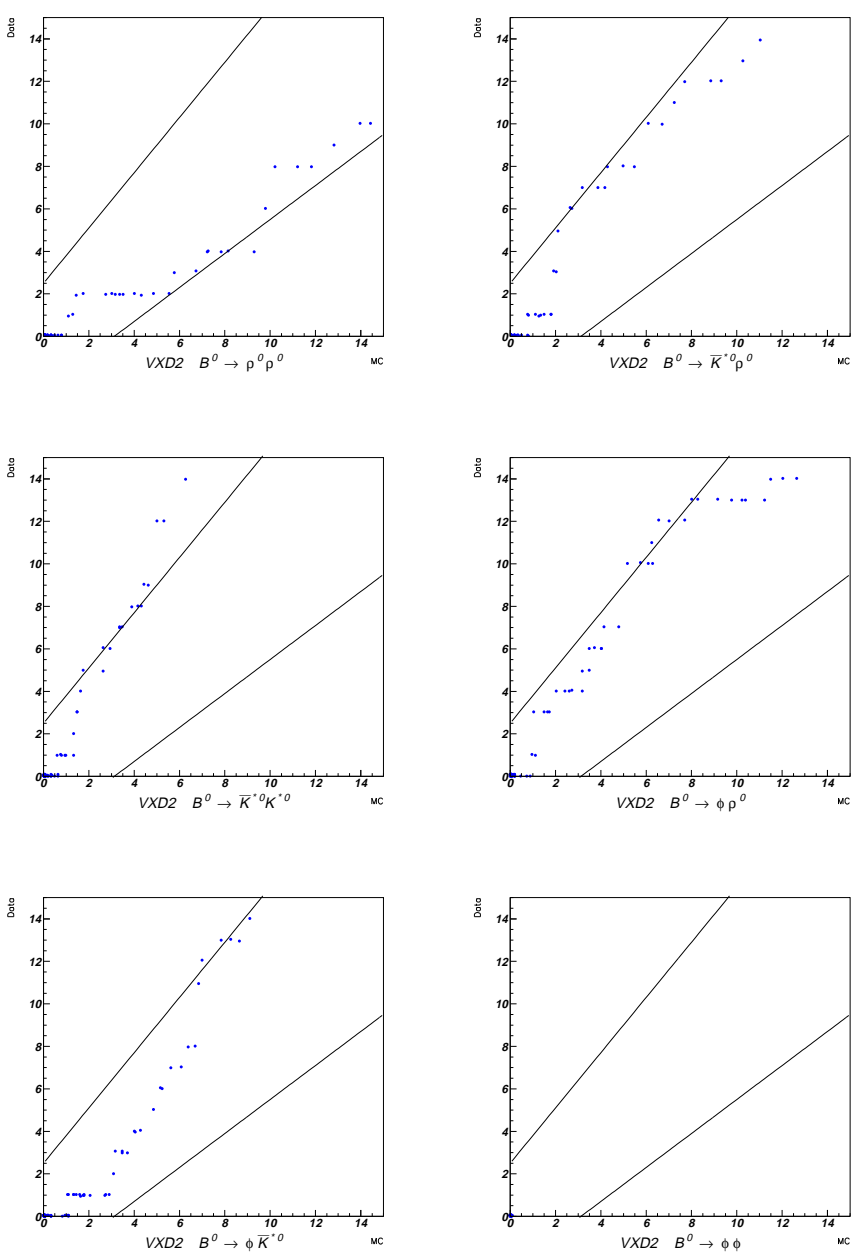

Figure B.23: Shown are the MC expected background level plotted versus the actual number of events seen in the data for VXD2 $B^{0} \rightarrow V V$. The lines indicates an approximately $90 \%$ confidence interval. 

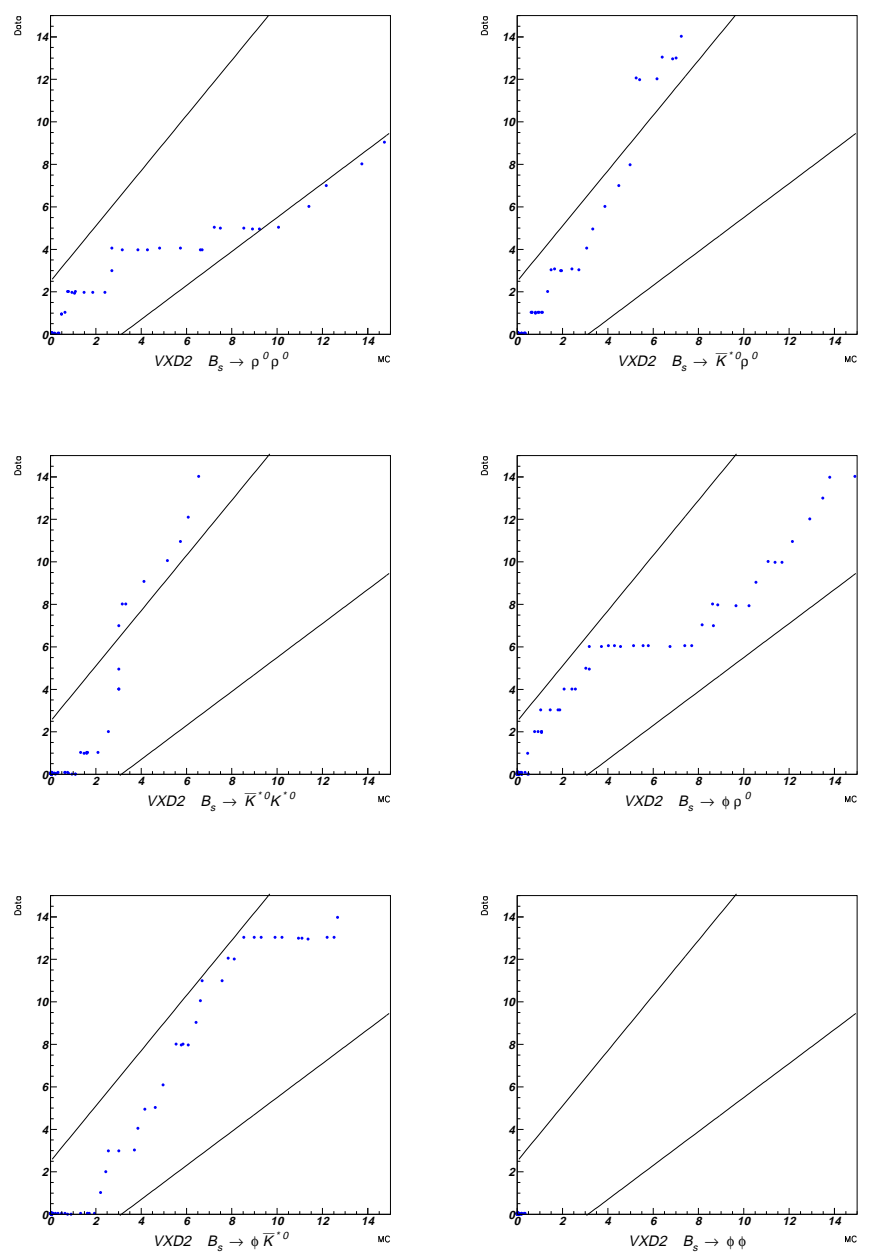

Figure B.24: Shown are the MC expected background level plotted versus the actual number of events seen in the data for VXD2 $B_{s} \rightarrow V V$. The lines indicates an approximately $90 \%$ confidence interval. 


\section{Backup Plots Associated with the Main Text}
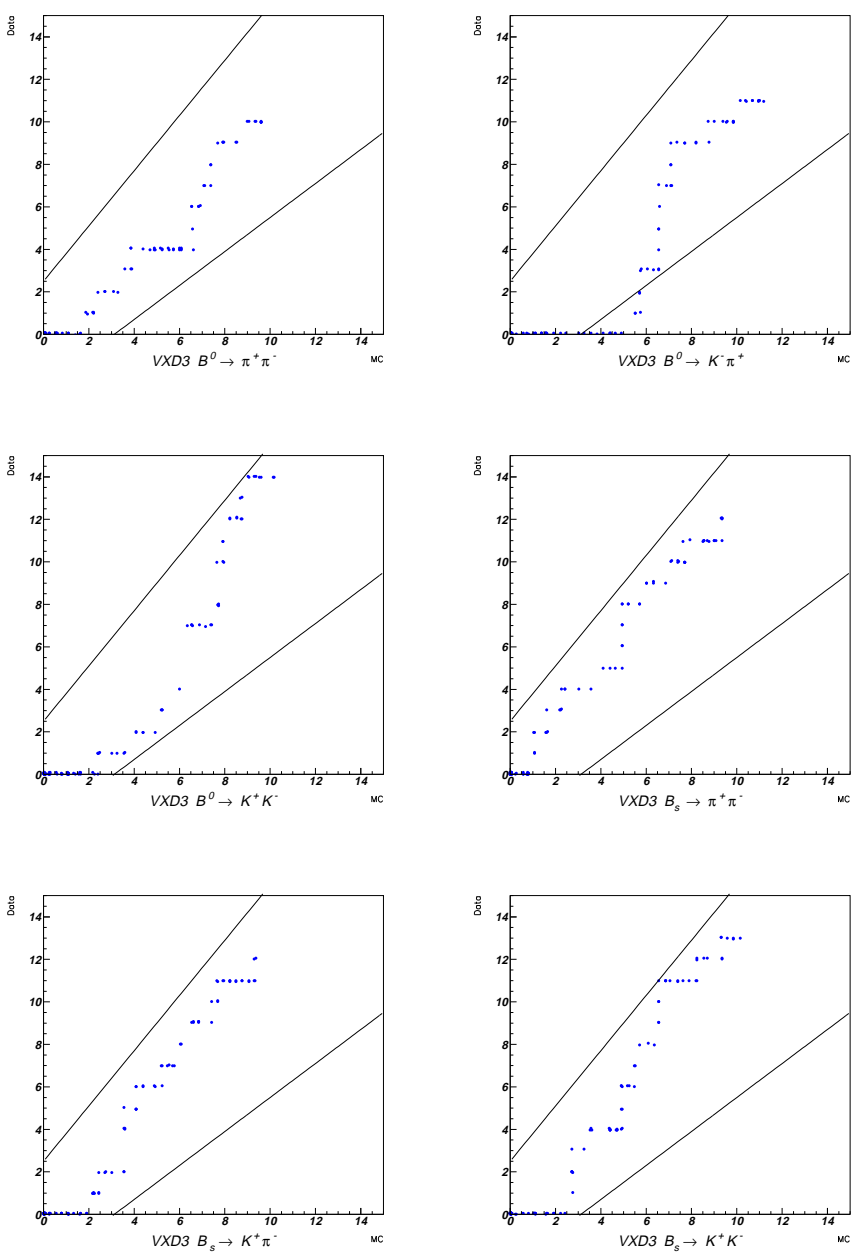

Figure B.25: Shown are the MC expected background level plotted versus the actual number of events seen in the data for VXD3 $B \rightarrow P P$. The lines indicates an approximately $90 \%$ confidence interval. 

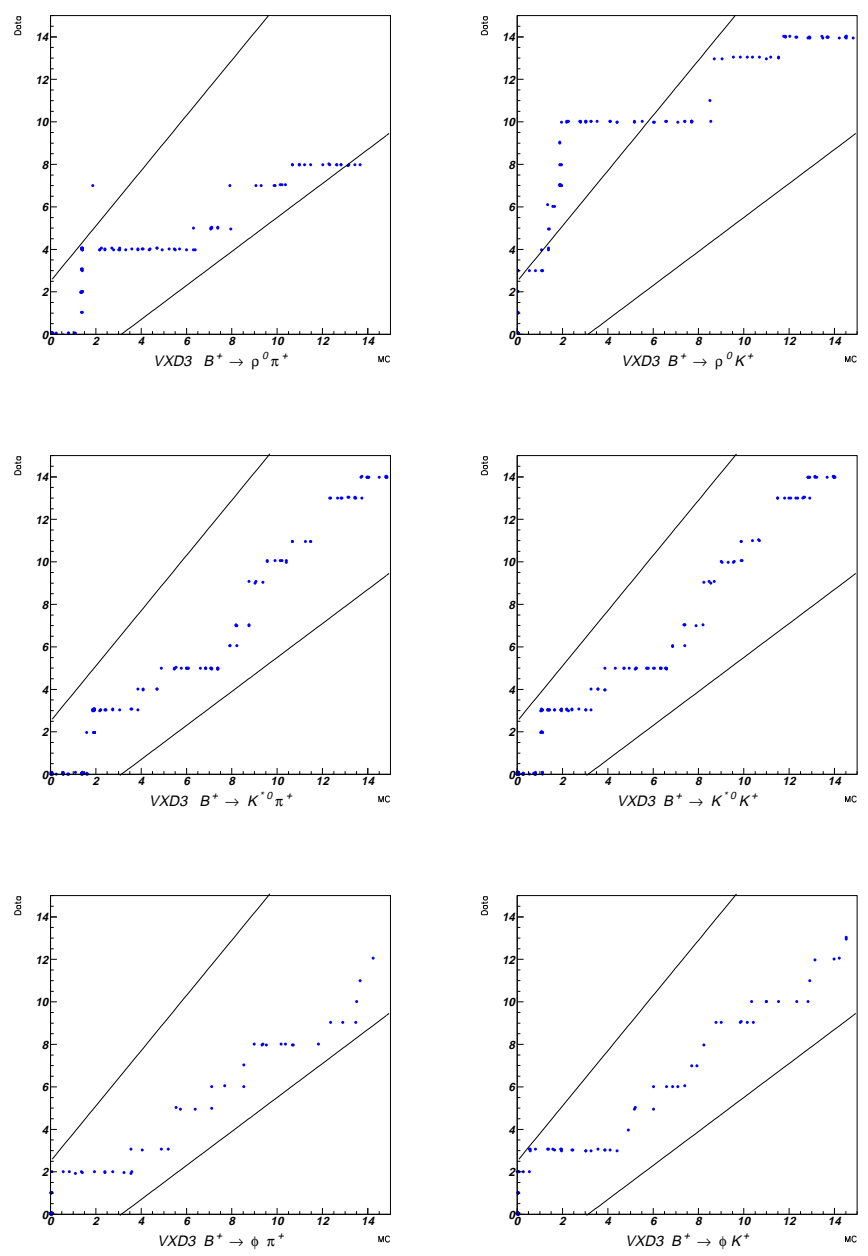

Figure B.26: Shown are the MC expected background level plotted versus the actual number of events seen in the data for VXD3 $B \rightarrow P V$. The lines indicates an approximately $90 \%$ confidence interval. 


\section{Backup Plots Associated with the Main Text}
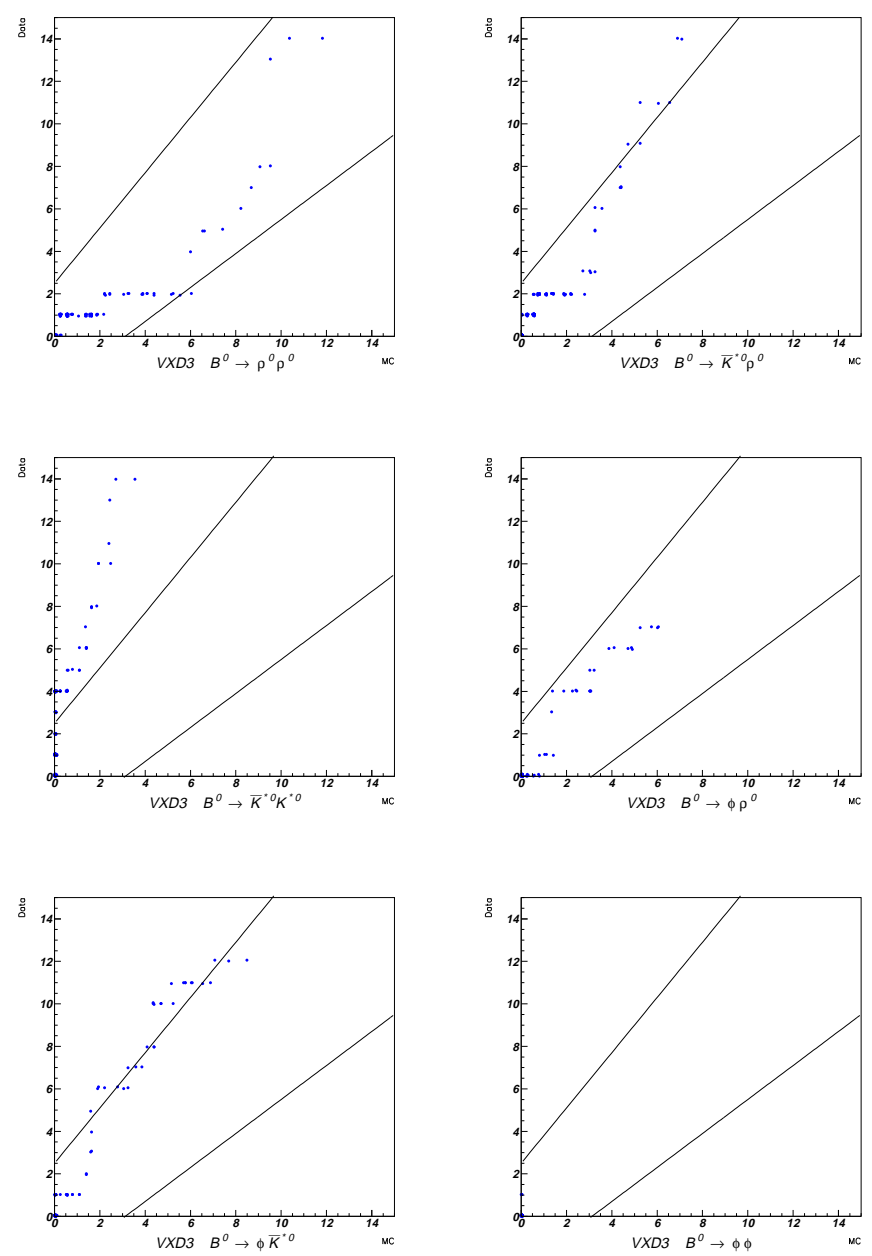

Figure B.27: Shown are the MC expected background level plotted versus the actual number of events seen in the data for VXD3 $B^{0} \rightarrow V V$. The lines indicates an approximately $90 \%$ confidence interval. 

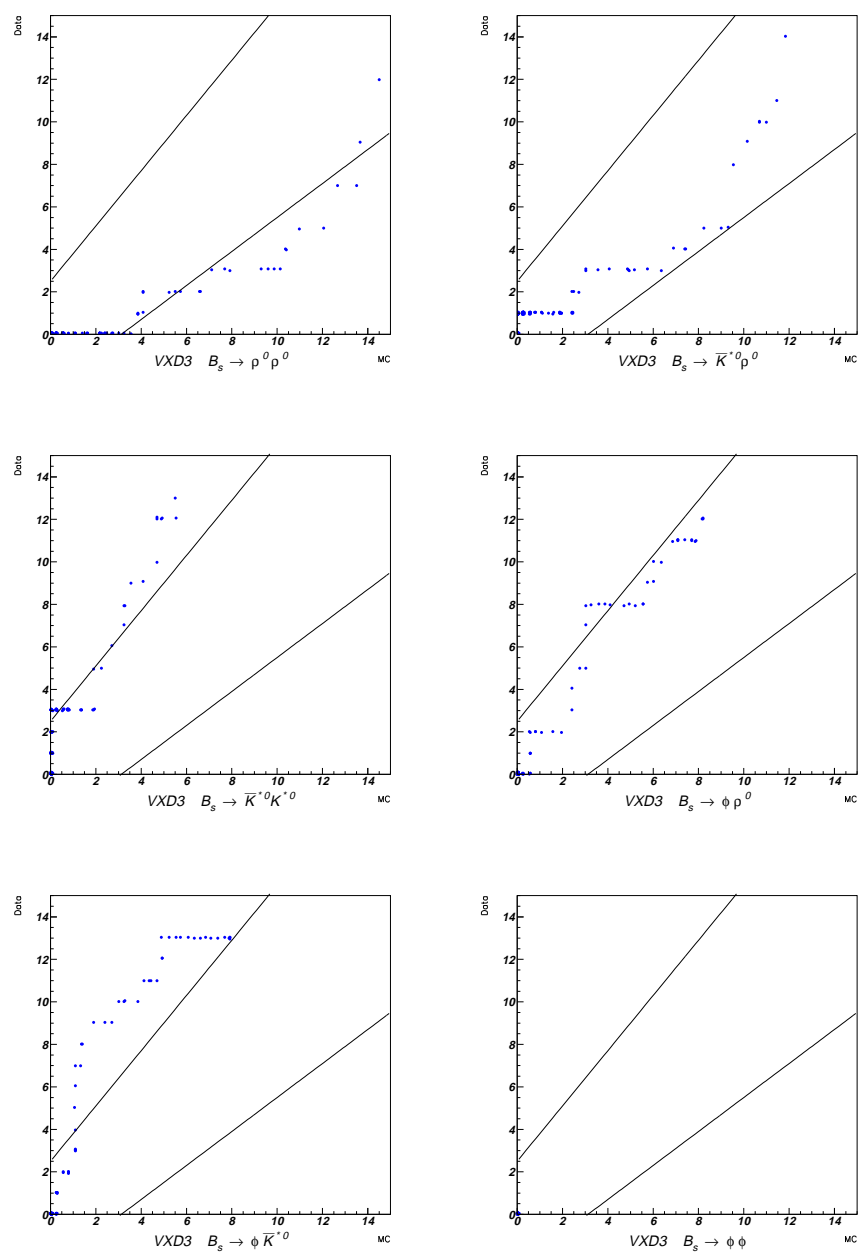

Figure B.28: Shown are the MC expected background level plotted versus the actual number of events seen in the data for VXD3 $B_{s} \rightarrow V V$. The lines indicates an approximately $90 \%$ confidence interval. 



\section{Bibliography}

[1] PDG Collaboration, The European Physical Journal C 3 (1998) 1-4.

[2] S.L.Glashow, Nucl. Phys. 22 (1961) 579;

A.Salam, J.C.Ward, Phys. Lett. 13 (1964) 168;

S.Weinberg, Phys. Rev. Lett. 19 (1967) 1264.

[3] O.Nachtmann, Elementary Particle Physics, Springer-Verlag (1990).

[4] F.Mandl, G.Shaw, Quantum Field Theory, John Wiley and Sons (1991).

[5] Y.Fukuda et al., Super-Kamiokande Collaboration, Phys. Rev. Lett. 82 (1999) 1810

[6] P.L.Reinertsen, SLAC-PUB-8029, (1998), To Be Published in Nucl. Phys. B.

[7] L.Wolfenstein, Phys. Rev. D 31 (1985) 2381

[8] M.Battle et al., CLEO Collaboration, Phys. Rev. Lett. 71 (1993) 3922.

[9] F.J.Yndurain, The Theory of Quark and Gluon Interactions, Springer-Verlag (1993).

[10] G.Bonneau et al., Nucl. Phys. B27 (1971) 381.

[11] E.A.Kuraev et al., Sov.J.Nucl.Phys. 41, 466 (1985).

[12] S.G.Gorishny et al., Phys. Lett. B 259 (1991) 114;

L.R.Surguladze et al., Phys. Rev. Lett. 66 (1991) 560. 


\section{BIBLIOGRAPHY}

[13] M.E.Peskin et al., Phys. Rev. D 46 (1992) 381.

[14] J.Bernabeu et al., Phys. Lett. B 363 (1991) 326.

[15] D.Bardin et al., ZFITTER, CERN-TH/92-6443 (1996).

[16] W.Hou, Nucl. Phys. B 308 (1988) 561.

[17] M.Gronau et al., Phys. Rev. D 52 (1995) 6356.

[18] D. Boutigny et al., Babar collaboration, SLAC-0443, 335pp (1994)

[19] L.Chau et al., Phys. Rev. D 43 (1991) 2176.

[20] H.Y.Cheng and B.Tseng, Phys. Rev. D 58 (1998) 094005.

[21] M.Daoudi, For the SLD Collaboration, SLAC-PUB-7941, (1998)

[22] F.E.Close et al., Phys. Rev. D 57 (1998) 5653.

[23] Y.H.Chen et al., Phys. Rev. D 59 (1999) 074003;

M.Gronau et al., Phys. Rev. D 58 (1998) 113005;

A.Ali et al., Phys. Rev. D 58 (1998) 094009;

A.Deandrea et al., Phys. Lett. B 320 (1994) 170;

L.L.Chau et al., Phys. Rev. D 43 (1991) 2176.

[24] Y.Gao et al., for the Cleo Collaboration, DPF99,session 3A, Jan. 6. 1999 at UCLA;

Y.Gao et al., for the Cleo Collaboration, hep-ex/9904008 (1999)

[25] W.Adam et al., Delphi Collaboration, Z. Phys. C 72 (1996) 207

[26] Aleph Collaboration, Phys. Lett. B 384 (1996) 471.

[27] CLEO Collaboration, Phys. Rev. Lett. 49 (1982) 357.

[28] SLD Collaboration, Phys. Lett. B 386 (1996) 475.

[29] Delphi Collaboration, Phys. Lett. B 347 (1995) 447

[30] Opal Collaboration, Z. Phys. C 53 (1992) 539

[31] SLD Collaboration, Phys. Rev. Lett. 81 (1998) 942. 
BIBLIOGRAPHY

[32] R. A. Fisher, Annals of Eugenics 7 (1936) 179.

[33] A.G.Frodesen et al., Probability and Statistics in Particle Physics, Universitetsforlaget (1979).

[34] "The SLD Design Report", SLAC-Report-229, (1980).

[35] R.Alley et al., Nucl. Instrum. Methods A365 (1995) 1.

[36] M.G.Minty, R.Akre, P.Krejcik, R.H.Siemann, Particle Accel. Conf: IEEE 1995:3046.

[37] R.Pitthan et al., Particle Accel. Conf: IEEE 1991:2098.

[38] T.L.Barklow et al., Part. Accel. 30 (1990) 121.

[39] J.G.Judkins et al., SLAC-PUB-3610, (1985).

[40] R.Erickson, SLAC-PUB-4479, (1987).

[41] P.Raimondi et al., SLAC-PUB-7955, (1998).

[42] T.Maruyama et al., Phys. Rev. Lett. 66 (1991) 2351.

[43] T.Maruyama, R.Prepost, Annual Review of Nucl. Part. Sci. 45 (1995) 41.

[44] C.Adolphson et al., SLAC-PUB-6118, (1993).

[45] T.Limberg et al., SLAC-PUB-6210, (1993).

[46] J.P.Fernandez, SLD Physics Note 258, (1999).

[47] G.Blaylock, SLC Physics Note 22, (1993).

[48] P.L.Reinertsen, SLD Physics Note 45, (1996).

[49] P.L.Reinertsen, SLD Note 261, (1999).

[50] R.C.Field et al., IEEE Transactions on Nuclear Science 45 (1998) 670.

[51] D.Onoprienko, talk at SPIN98, Protvino Russia Sep. 1998.

[52] M.Fero, P.L.Reinertsen et al., SLD Physics Note 50, (1996); 
[53] "The SLD Design Report", SLAC-Report-273, (1984).

[54] K.Pitts, SLAC-Report-446, (1994).

[55] M.B.Smy, SLAC-Report-515, (1997).

[56] C.J.S.Damerell et al., Nucl. Instrum. Methods 213 (1981) 201.

[57] M.D.Hildreth et al., Nucl. Instrum. Methods A367 (1995) 111.

[58] D.Williams, PhD Thesis, Massachusetts Institute of Technology (1994).

[59] K.Abe et al., Nucl. Instrum. Methods A343 (1994) 74.

[60] T.J.Pavel, PhD Thesis, Stanford University (1996).

[61] D.Axen et al., Nucl. Instrum. Methods A328 (1993) 472.

[62] A.C.Benvenuti et al., Nucl. Instrum. Methods A290 (1990) 353.

[63] D.Sherden, Nucl. Instrum. Methods A265 (1988) 342.

[64] By Torbjorn Sjostrand, Comput. Phys. Commun. 82 (1994) 74.

[65] R. Brun, R. Hagelberg, M. Hansroul, J.C. Lassalle, CERN-DD/72/2 (1978).

[66] P.N.Burrows et al., SLD Note 229, (1993).

[67] C.Peterson et al., Phys. Rev. D 27 (1983) 105.

[68] E.Fahri, Phys. Rev. Lett. 39 (1587) 1977.

[69] Private discussions with Stephan Willocq and David Muller.

[70] Electron ID: Private discussion with Salvatore Fahey.

Muon ID: Private discussion with Giampiero Mancinelli.

[71] R.D.Cousins et al., Nucl. Instrum. Methods A320 (331) 1992

[72] G.J.Feldman et al., Phys. Rev. D 57 (3873) 1998

[73] J.Neyman, Philos. Trans. R. Soc. London A236, (1937), 333. 